\title{
Learning Prerequisites for Biodiversity Education -
}

Chilean and German Pupils' Cognitive Frameworks and Their Commitment to Protect Biodiversity

\author{
Dissertation \\ zur Erlangung des Doktorgrades \\ der Mathematisch-Naturwissenschaftlichen Fakultäten \\ der Georg-August-Universität zu Göttingen
}

vorgelegt von

Susanne Menzel

aus Siegen

Göttingen 2007 
D 7

Referentin: Prof. Dr. Susanne Bögeholz

Korreferent: Prof. Dr. Marcus Hasselhorn

Tag der mündlichen Prüfung: 05.07.2007 
"In the end we will conserve only what we love.

We will love only what we understand.

We will understand only what we are taught.”

Baba Dioum,

Senegalese poet and environmentalist 



\section{Acknowledgements}

During the last three years, when this dissertation came into being, numerous colleagues, friends, and my family supported me. Without them, my work would have been more difficult, but, above all, my life would have been much poorer.

First, I would like to thank my advisor, Prof. Dr. Susanne Bögeholz for funding, help, support and inspiration. Her guidance has helped me to cross borders, literally - and geographically. I would also like to express my gratitude to Prof. Dr. Marcus Hasselhorn, my second advisor, for his help and support, especially during the last phase of this dissertation. Furthermore, I am indebted to the following people for being members of my examination committee: Prof. Dr. Leuschner, Prof. Dr. Heymann, Prof. Dr. Schäfer and Prof. Dr. SchulzHardt. Many thanks also to Prof. Dr. Jens Naumann for reminding me to do a Ph.D. and, above all, for sparking my interest for a "southern"-perspective on the world.

My work in South America was supported by numerous wonderful people. Gracias, Dr. Luís Astudillo, for your never-ending support and help, and for sacrificing your precious time. ¡Gracias a todos del laboratorio por su ayuda y amistad! Dr. Marisol Cazar, thank you for helping me in countless occasions, for being a wonderful host, and friend. In the same regard, I am much obliged to Giancarla Solari, Enrique, Susana, Rey and Paula Zambra; what would I have done without your help? My time in Chile and Ecuador will always be remembered as time spent among friends.

I am particularly grateful to Christina Schönborn and Dr. Konrad Schönborn for their friendship and help - and patience with numerous English drafts of this work. Dr. Christina Rüffer, Dr. Jan Freese, Sabina Eggert, Olaf Munisso, Melanie Herget, and, particularly, Oliver Konrad for their patience, support, helpful comments on earlier drafts of this work and, above all, for their friendship. Thank you, too, my friends Sandra Rajmis, Jiong Yan and Claudia Cerda for spending wonderful times with me in Göttingen! In addition to the above mentioned people, I must also acknowledge my marvellous colleagues in Göttingen; Christa Grass, Stephanie Rüter, Janin Schatz, Nina Holstermann, Sylvia Leske, Silvia Schönfelder, Susanne Kreuter and Sevilay Dervişoğlu for their support - and especially for bearing with me on Monday mornings ;o) Thanks also to Dr. Thorsten Roick for his help 
with administrative steps to be taken and advice on empirical questions. Special thanks go to my student assistant, Michaela Volle, for her valuable help and her reliable work.

To name all the friends who were there for me, either personally, or in mail or telephone format, would fill pages. Thank you, my "Dortmund" friends, the "Kollektief" and the "Toubabs" in Köln.

Above all, I am especially grateful to my beloved family, Regina, Karl-Heinz, Mario and Kathrin Menzel plus Vali, Milli and Oliver Link, Irmgard Jung, the Schölers, Erna Menzel and all the (many) others belonging to the extended "Wittgenstein-Clan". Thanks for supporting me - and for being there, today and during my happy childhood, when the foundations for my love to nature were laid.

Finally, I would like to say thank you to Raphael Riedl. Your unquestioning presence and love has, always, enabled me to do what I do, as best as I can possibly do it.

Göttingen in May 2007,

Susanne Menzel 




\section{Outline}

Acknowledgements...........................................................

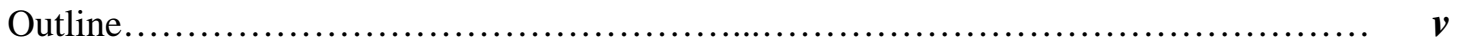

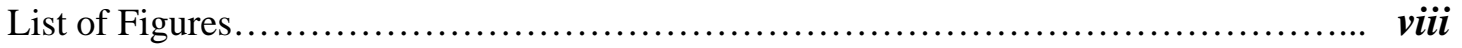

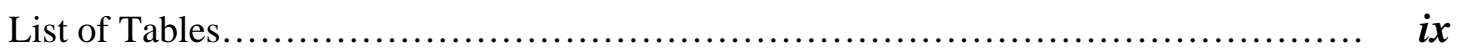

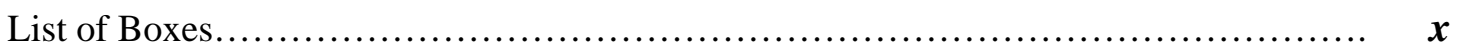

List of Acronyms..................................................................

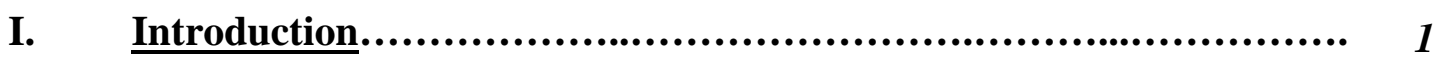

$1 \quad$ Sustainable Development.................................................. $\quad 2$

1.1 Synthesis of a definition: sustainable development................... 3

1.2 Central environmental and developmental challenges................ 6

$2 \quad$ What is Biodiversity? ...................................................... 7

2.1 Defining biodiversity ............................................ 7

2.2 Biodiversity hotspots......................................... 9

2.3 Resource dilemmas.......................................... 10

2.4 Education as a mandatory precondition for biodiversity conservation. 11

3.1 Cognitive frameworks as learning prerequisites for biodiversity: research focus I.

3.2 Factors influencing pupils' commitment to protect biodiversity: research focus II.

II. Empirical Part.

4 Lernvoraussetzungen für Biodiversity Education in Deutschland und Chile am Beispiel endemischer Medizinalpflanzen....

4.1 Einleitung

4.2 Biodiversity Education - eine Herausforderung für die

Biologiedidaktik.....

4.3 Untersuchungsaufbau und Methoden. 
4.4 Erste Ergebnisse.............................................. 35

4.5 Diskussion und Ausblick....................................... 38

5 Caminos empíricos en la investigación didácitica para mostrar la necesidad de una enseñanza transdisciplinaria sobre la biodiversidad...... 41

5.1 Abstract................................................... 41

5.2 Introducción................................................. 42

5.3 Biodiversidad: téma urgente y complejo......................... 43

5.4 Preguntas de investigación..................................... 43

5.5 Metodología................................................. 44

5.6 Resultados.................................................... 45

5.7 Implicaciones................................................ 48

6 Vorstellungen und Argumentationsstrukturen von Schüler(inne)n der elften Jahrgangstufe zur Biodiversität, deren Gefährdung und Erhaltung 51

6.1 Zusammenfassung/Abstract................................ 51

6.2 Biodiversitätsverlust als Herausforderung für eine Bildung für Nachhaltige Entwicklung.................................. 52

6.3 Theoretischer Hintergrund, Stand der Forschung und Forschungsfragen........................................ 53

6.4 Untersuchungsaufbau und Methoden............................ 56

6.5 Ergebnisse............................................... 61

6.6 Diskussion - Schülervorstellungen zur Biodiversität als Herausforderung für eine Bildung für eine Nachhaltige Entwicklung... $\quad 71$

7 The Loss of Biodiversity: How do Students in Chile and Germany Perceive Threats and Which Possible Solutions Do They See?.............. 75

7.1 Abstract.................................................... 75

7.2 Introduction................................................... 75

7.3 Theoretical background.................................... 77

7.4 Research questions........................................ 80

7.5 Methods...................................................... 80

7.6 Results.................................................... 86

7.7 Discussion..................................................... 96

8 Was fördert die Bereitschaft von Oberstufenschüler(inne)n, die Biodiversität zu schützen? - Eine quantitative Studie in Anlehnung an die Value-Belief-Norm Theorie...................................................... 107

8.1 Zusammenfassung und Abstract................................ 107

8.2 Fragestellung................................................. 108 
8.3 Methodik.................................................... 113

8.4 Ergebnisse.................................................. 117

8.5 Diskussion................................................... 120

9 Values, Beliefs and Norms That Foster Chilean and German Pupils' Commitment to Protect Biodiversity................................... 125

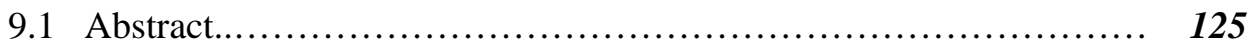

9.2 Introduction................................................... 125

9.3 Theoretical framework......................................... 126

9.4 Research questions and hypotheses............................. 127

9.5 Methods..................................................... 128

9.6 Results..................................................... 130

9.7 Discussion.................................................... 134

9.8 Conclusions and implications for education..................... 137

III. Summary and Discussion........................................ 139

10 Pupils' Cognitive Frameworks and their Commitment to Protect Biodiversity...................................................................... 141

10.1 Cognitive frameworks of pupils in Chile and Germany (research focus I)....................................... 141

10.2 Chilean and German pupils’ commitment to protect biodiversity (research focus II).

10.3 Critical Discussion............................................ 155

11 Educational Implications and Concluding Remarks........................ 157

12 References........................................................................ 163

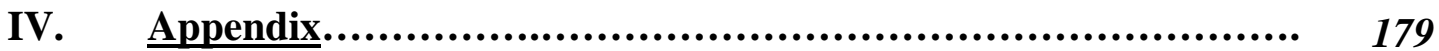

A Interview Guide of the Qualitative Interview Study (German and Spanish Version). 181

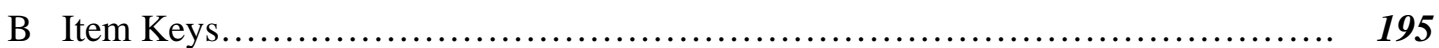

C Questionnaire of the Quantitative Study (German)............................. 199

D Abstracts (English and German) ..................................... 227

E Curriculum Vitae.......................................................... 229 


\section{List of Figures}

Figure 1.1

Figure 2.1

Figure 3.1

Figure 3.2

Figure 3.3

Figure 4.1

Figure 5.1

Figure 5.2

Figure 5.3

Figure 5.4

Figure 6.1

Figure 6.2

Figure 6.3

Figure 6.4

Figure 7.1

Figure 7.2

Figure 7.3

Figure 7.4

Figure 8.1

Figure 9.1
An integrated concept of sustainable development

The 34 biodiversity hotspots in the world

The structure of cognitive frameworks after Groeben et al.

A schematic representation of Schwartz' NormActivation-Model of altruistic behaviour (VBN) theory

Wert und Gefährdung der Biodiversität

Guía de entrevista

El típo ecológico

46

El típo social “desarrollo sostenible”

47

Inhaltlicher Aufbau des Interview Leitfadens

59

Beispiele für induktiv angelegte Kategorien zu Schülervorstellungen zur Biodiversität und biologischen Vielfalt

Beispiele für deduktiv angelegte Kategorien zu ökologisch-sozialen Dilemmata Phasen des Interviews during the first step of the interview

The economical type

The balanced type

Erweitertes Modell zur Erklärung von Bereitschaften von Oberstufenschüler(inne)n, Biodiversität zu schützen, angelehnt an die VBN Theorie

Model to explain adolescents' commitment to 


\section{List of Tables}

Table 4.1

Table 4.2

Table 6.1

Table 6.2

Table 6.3

Table 7.1a

Table 7.1b

Table 7.2

Table 7.3

Table 8.1

Table 8.2

Table 8.3

Table 8.4

Table 8.5

Table 9.1

Table 9.2

Table 9.3

Table 9.4

Table 13.1

Table 13.2
Erhebungen von Lernvoraussetzungen von

Schülerinnen und Schülern

Forschungsinteresse der explorativen Studie mit Beispielinterventionen

34-35

Zusammensetzung der [qualitativen] Stichprobe 57

Auszüge aus dem Interview Leitfaden

Kriterien für die Bildung der Typen

68

German Sample used in the interviews

82

Chilean Sample used in the interviews

82

Three-step structure of the interview protocol used in the study

83

Typology, criteria and pupils' typological affiliation

92

Die universellen menschlichen Werte nach Schwartz

109

Konstrukte und Beispielitems

114-115

Deskriptive Kennwerte der im Fragebogen eingesetzten Skalen

118

Regressionen für die Bereitschaft zu drei verschiedenen Handlungstypen

Gesamtmodell für die Bereitschaft, Biodiversität durch Aktivismus, nichtaktivistisches, öffentliches Handeln und Handeln im privaten Raum zu schützen

Sources of scales as used in the study

Comparison between means of the German and Chilean sample

130-131

Results of regression analyses for the Chilean sample

Results of regression analyses for the German sample

Items of the questionnaire part I

Items of the questionnaire part II 


\section{List of Boxes}

Box 1.1

Box 2.1
The WCED's definition of sustainable

4 development

A definition of biodiversity 


\section{List of Acronyms}

$\begin{array}{ll}\text { AC } & \text { Awareness of Consequences } \\ \text { AR } & \text { Ascription of Responsibility } \\ \text { CBD } & \text { Convention on Biological Diversity } \\ \text { CDLA } & \text { Consejo de las Américas } \\ \text { DNA } & \text { Desoxyribosenucleic Acid } \\ \text { IUCN } & \text { International Union for the Conservation of Nature and Natural } \\ & \text { Resources } \\ \text { NAM } & \text { Norm-Activation-Model } \\ \text { NEP } & \text { New Ecological Paradigm / New Environmental Paradigm } \\ \text { PA } & \text { Perceived Ability to Reduce Threats to the Environment } \\ \text { PN } & \text { Personal Norms } \\ \text { PVQ } & \text { Portrait Value Questionnaire } \\ \text { UNCED } & \text { United Nations Conference on Environment and Development (“Rio } \\ & \text { Conference”, Rio de Janeiro 1992) } \\ \text { UNECE } & \text { United Nations Economic and Social Council } \\ \text { UNEP } & \text { United Nations Environnemental Programme } \\ \text { UNESCO } & \text { United Nations Educational, Scientific and Cultural Organization } \\ \text { VBN } & \text { Value-Belief-Norm } \\ \text { WCED } & \text { World Commission on Environment and Development } \\ \text { WCS } & \text { World Conservation Strategy } \\ \text { WSSD } & \text { World Summit on Sustainable Development ("Earth Summit”, } \\ \text { WWF } & \text { World Wildlife Fund for Nature } \\ & \end{array}$





\section{Introduction}





\section{Introduction}

At the beginning of this $21^{\text {st }}$ century, our world faces numerous social and environmental problems (cf. UNCED, 1992; WCED, 1987). Most of these problems, such as climate change, poverty, the unequal distribution of wealth, freshwater shortage and species extinction are closely interrelated. Therefore, within the last thirty years, a worldwide claim for sustainable development has emerged, i.e. a social, economic, and ecological development that allows a continued survival of mankind on earth (UNCED, 1992).

The loss of biodiversity (or biological diversity) on the level of species, ecosystems and genes is among the most challenging environmental problems (e.g. Myers, 1985; Myers, N. Mittermeier, R.A. Mittermeier, da Fonseca \& Kent, 2000; Pullin, 2002; Shiva, 2000; Wilson, 1992). However, biodiversity loss is not an exclusively ecological issue, neither in its causes, nor in its consequences. Although the term "biodiversity” might suggest an ecological focus, social and economic factors play a central role in biodiversity loss and protection. Particularly in so-called "biodiversity hotspots", regions in which biodiversity is exceptionally threatened, social and economic interrelations become evident (Cincotta, Wisnewski \& Engelman, 2000; N. Mittermeier, R.A. Mittermeier, da Fonseca \& Kent, 2004; Myers et al., 2000). As a central measure to protect biodiversity in the sense of sustainable development, numerous authors and international legal frameworks have claimed the inclusion of the topic in educational programmes - on an academic as well as an extracurricular level (e.g. Bell, Russell \& Plotkin, 1998; CBD, 1992; Dreyfus, Wals \& van Weelie, 1999; Kassas, 2002; du Plessis, 2001; UNCED, 1992). Schools play a central role in this respect. However, little evidence exists so far about how educational measures should be designed in order to be fruitful. The author identified two basic challenges for biodiversity education research that will be addressed in this dissertation.

Firstly, little is known so far about how learners perceive the problem of biodiversity loss, let alone if they are able to identify the complex social and economic relations that are associated with the topic. However, in a constructivist perspective such information would be helpful as learners cannot be seen as passive recipients of information. Rather, learners are active constructors of knowledge (Driver, 1989). Therefore, previous perspectives are important determents for successful learning. Thus, within the first research focus of this thesis (research focus $I$ ), an exploratory study was conducted to assess existing cognitive frameworks of pupils on biodiversity, and biodiversity loss. 
Secondly, little is known about pupils' actual commitment to protect biodiversity. In order to foster conservational activities, it is extremely helpful to have evidences at one's disposal about which factors are inhibiting or fostering a commitment to protect biodiversity. Therefore, a second research focus assessing psychological factors that influence a commitment to protect biodiversity (research focus II) was conducted in conjunction with exploring cognitive frameworks within the research focus I. Although being highly complementary, both research foci demanded different theoretical and methodological approaches. As a consequence, research focus I was a qualitative interview study, while for research focus II quantitative methods were applied.

In a third research interest both foci were spanned through an intercultural comparison. Thus, pupils who live at a biodiversity hotspot in a developing country were compared to pupils living in an industrial country with a medium level of biodiversity and biodiversity loss. In order to achieve this, both research foci were realized in two countries, namely Chile and Germany.

In the following introductory chapter of this dissertation (I), a recap on how the concept of sustainable development emerged will be provided (1.1). Then, central challenges for sustainable development will be summarized (1.2). Within a second chapter, biodiversity will be defined (2.1), and the issues of biodiversity hotspots (2.2) and resource dilemmas (2.3) will be addressed before the central role that education plays will be outlined (2.4). The introduction closes with overall questions and hypotheses of the dissertation (chapters 3.1 and 3.2).

\section{Sustainable Development}

"In the middle of the $20^{\text {th }}$ century, we saw our planet from space for the first time. [...] From space, we see a small and fragile ball dominated not by human activity and edifice but by a pattern of clouds, oceans, greenery, and soils. Humanity's inability to fit in its doings into that pattern is changing planetary systems, fundamentally. Many such changes are accompanied by life-threatening hazards. This new reality, from which there is no escape, must be recognized and managed."

(WCED, 1987, p. 1) 
Twenty years have passed since the World Commission on Environment and Development's (WCED) Brundtland-Report ${ }^{1}$ was published, where the above cited statement can be found in the introduction. However, humanity still faces the developmental and environmental challenges that were first defined by the report, and much work remains to be done to achieve what the report claims: sustainable development.

\subsection{Synthesis of a definition: sustainable development}

In order to understand sustainable development, it is helpful to briefly consider how the concept emerged and through which stages it went to finally be defined as can be found in the Brundtland report and, later, in the documents of the World Summits in Rio de Janeiro and Johannesburg.

To begin with, it is essential to understand that many of the antecedents of the concept of sustainable development stem from forest and wildlife management (Redclift, 1987). Here, the focus was still on a practical and local scale: sustainability was a concept to safeguard harvests of natural resources, such as trees or crops. Consequently, the main thought behind these early concepts of sustainability was one of sustainable yield, thus, a determination of a particular amount of a resource that can be removed whilst allowing the respective resource or ecosystem to recover (Whitehead, 2007). In consequence, early concepts of sustainability did not yet take economic forces as part of a possible solution into account. Instead, the economy was seen as an antagonist that had to be restricted in order to ensure a sustainable management of natural resources (Whitehead, 2007).

Economic development was implicated first as a possible asset to conserve natural resources when the International Union for the Conservation of Nature and Natural Resources (IUCN) published its World Conservation Strategy (WCS) in 1980 (IUCN, 1980; Whitehead, 2007). Here, "sustainable development was codified for the first time" in that the economy was taken as a possible part of a solution into account (Adams, 2001, p. 54). Although being heavily criticized for a) being too focused on human well-being, and therefore not taking an intrinsic value of nature into account and b) for ignoring the harm that economy and current political practices had done to nature, the WCS imbued economical development with environmental ideas and principles (Adams, 2001; Whitehead, 2007). In this regard, the WCS moved further than most of its previous concepts of forest and wildlife management. However, the World Conservation Strategy still provided little practical advice for actual

\footnotetext{
${ }^{1}$ Original title: "Our Common Future". However, the report is also commonly named after the WCED's Norwegian chairman, Gro Harlem Brundtland.
} 
environmental policy. Moreover, no clear position emerged within the document indicating in which way the two sectors (environmental and economic issues) should be weighted (Adams, 2001).

The next milestone document, the afore-mentioned Brundtland-Report, again went one step further. In this document, the WCED laid the foundations for today's definition of sustainable development. The central aspect of the WCED's definition is the explicit embedding of the needs of future generations into the concept (see box 1.1).

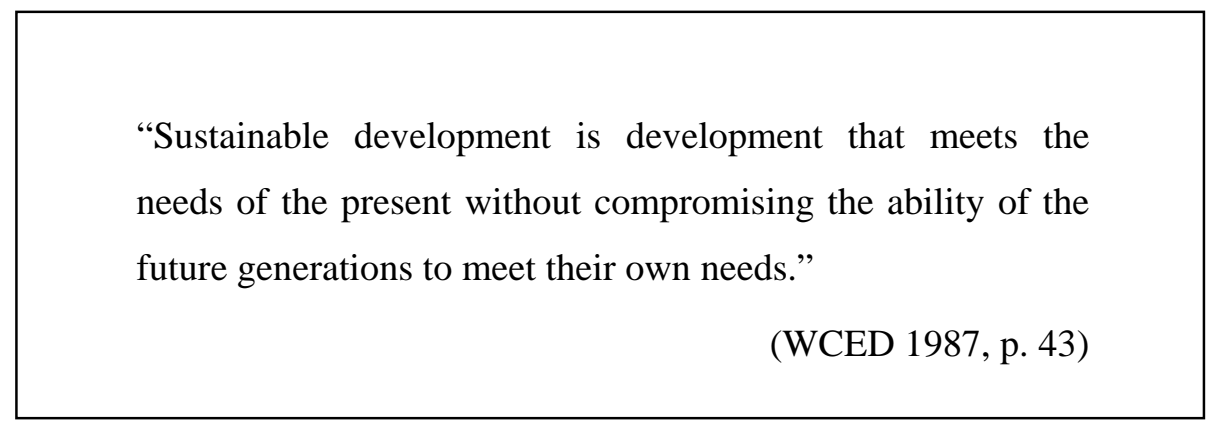

Box 1.1. The WCED's definition of sustainable development.

Thus, a social perspective was taken in addition to ecological and economic considerations for sustainable development. Ecological and economic development were integrated by considering "merging environment and economics in decision making" as one out of seven strategic imperatives formulated in the report (WCED, 1987, p. 49ff). Therefore, by taking i) ecological, ii) economic, and iii) social issues into account, the foundations for the threepillar-concept of sustainable development were formally laid within the Brundtland Report. Moreover, the report clearly included proposals for institutional and legal changes to achieve the goals of sustainable development, of which broad suggestions for the implementation of the concept of sustainable development were offered (WCED, 1987, p. 308ff).

To report progress on these suggestions made by the WCED, the United Nations General Assembly held a conference five years after the publication of the Brundtland-Report. This conference, the United Nations Conference on Environment and Development (UNCED), took place in Rio de Janeiro in 1992. During preparations of the Rio-Conference, it became evident that more practical and concrete guidelines needed to be developed to succeed with a global implementation of the sustainable development concept. A document was needed that went beyond the still broad and general guidelines that were provided by the BrundtlandReport. Therefore, a global action plan for sustainable development in the twenty-first century was prepared and ratified as one of the central outputs of the UNCED: the Agenda 21 (UNCED, 1992). The core idea of Agenda 21 is the self-commitment of governments to 
adopt national strategies for sustainable development. According to the document, each member state should be able to design its own strategy to integrate environmental considerations into the nation's overall economic and social development (Terry, Hill \& Woodland, 2006). By doing so, neither Agenda 21, nor the three other central output documents of the UNCED (the Forest Principles, the Framework Convention on Climate Change, and the Convention on Biological Diversity) left any doubt that the interaction of economy, ecology and social issues is one core aspect of sustainable development. This idea prevailed and also formed the basis of the outputs of the World Summit for Sustainable Development (WSSD) in Johannesburg in 2002, ten years later.

The three-pillars-concept of sustainable development in the tradition of WCED, UNCED and WSSD has often been criticized for being too imprecise, too weak and not powerful enough (cf. Adams, 2001; Pepper, 1993; Tainter, 2003; Worster, 1993). As a consequence, alternative and often more practical concepts for sustainable development have been suggested (cf. Allen, Tainter \& Hoekstra, 1999; Allen, Tainter \& Hoekstra, 2003; Kopfmüller et al., 2001). However, if we try to bring these different concepts together, it would probably arrive at a minimum consensus that presents as an integrated concept and which, in return, falls back on the concept of three pillars of sustainable development (Whitehead, 2007) (see figure 1.1).

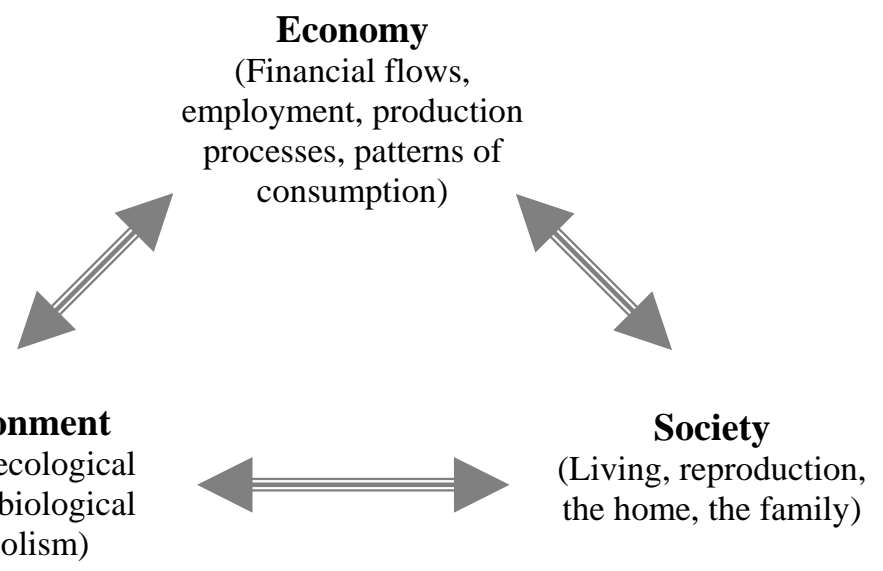

Fig. 1.1. An integrated concept of sustainable development (after Whitehead, 2007, p. 14). 
We can resume that the general ambition of the reflection of all the three pillars of economy, ecology and social issues (the latter including the needs of future generations) prevails and will, thus, serve as a basis in the understanding of sustainable development in this dissertation.

\subsection{Central environmental and developmental challenges}

What are the challenges of sustainable development? From the beginning, when the concept of sustainable development began to emerge, the topic of the preservation of species took centre stage. For instance, the World Conservation Strategy (WCS; IUCN, 1980) formulated three main principles. All of these principles explicitly referred to aspects of species preservation. The first principle expresses the necessity to "maintain essential ecological processes and life-support systems [...]" (IUCN, 1980). The second principle takes a different perspective on the conservation of species by claiming a preservation of genetic diversity, i.e. the variety within species. Finally, the third principle addresses the issue of profits from species and ecosystems and claims a sustainable use of the respective resources (IUCN, 1980). Twelve years later, the extinction of species was one of the central topics of the UNCED Rio conference in 1992. According to this focus, the Convention on Biological Diversity (CBD) can be regarded as one key agreement adopted in Rio. Presently, 190 countries have ratified the CBD, which is the majority of the world's governments. The status of biodiversity conservation as a central challenge for sustainable development has herewith been clearly identified and this affirms the central position that the topic of biodiversity had already achieved in the World Conservation Strategy in 1980.

Nevertheless, there are other challenges besides biodiversity protection. Some authors suggest classifications as the most important of these challenges. For instance, Hesse (2006) defines the "Big Six for Sustainable Development", as 1. Climate change, 2. Freshwater shortage and pollution, 3. Deforestation and desertification, 4. Poverty, 5. Migration and population growth and 6. Biodiversity loss. In doing so, Hesse explicitly mentions biodiversity as one challenge out of six. However, the dominance of the topic is clearly shown as biodiversity is closely connected to all the challenges defined. For example, biodiversity loss can be caused by climate change due to species' inability to adapt to new climatic circumstances (Pounds, Fogden \& Campbell, 1999). Additionally, freshwater shortage and pollution can be caused by deforestation and desertification, which are in turn again threats to biodiversity. Poverty is a central factor for biodiversity loss because lack of income (especially in developing countries) can lead to overuse of natural resources for food 
and subsistence (i.e. to cover the basic need of income). Particularly when public natural resources are concerned, protection from overuse is difficult due to the necessity of beneficiaries to cover basic needs (Dietz, Ostrom \& Stern, 2003; Ernst, 1997; Hardin, 1968). Finally, migration and population growth lead to increasing urbanization with serious effects on environmental degradation in the suburban and surrounding areas. Poverty that often occurs on the outskirts of cities in developing countries, leads specifically to overuse of natural resources in areas close to the respective cities (Marshall, 1998).

With regards to the emergence of the concept of sustainable development, we can conclude that biodiversity and its loss i) have been at the centre of the challenges of sustainable development from the very beginning and ii) are closely connected to the other challenges of sustainable development.

\section{$2 \quad$ What is Biodiversity?}

\subsection{Defining biodiversity}

The term "biodiversity" is short for "biological diversity" and both terms are identical in meaning. In most languages, both expressions exist and are usually synonyms. It is unclear, however, when the term biodiversity was created. Most likely, it emerged within the early 1980’s and has since spread rapidly (Flitner, 1999). Presently, however, biodiversity has no strict scientific definition although the term is commonly used in both the scientific and political fields (Pullin, 2002). The simplest definition of biodiversity is "the variety of species”. Such a simple definition describes biodiversity as the number of species in a given area (species diversity). However, regarding species diversity exclusively neither broaches the biodiversity concept to its full extend (cf. Townsend, Begon \& Harper, 2003; Wilson, 2001), nor would concentration of the species level be sufficient for conservational efforts (Walker, 1992). Most modern definitions, therefore, consider biodiversity at three different levels (after Pullin, 2002, p. 6):

1. Genetic diversity (the diversity within-species in terms of genetic differences),

2. Species diversity (the combination of the number and evenness of abundance of species),

3. Community or ecosystem diversity (the number of different species assemblages). 
The same underlying idea can also be expressed by the following formula:

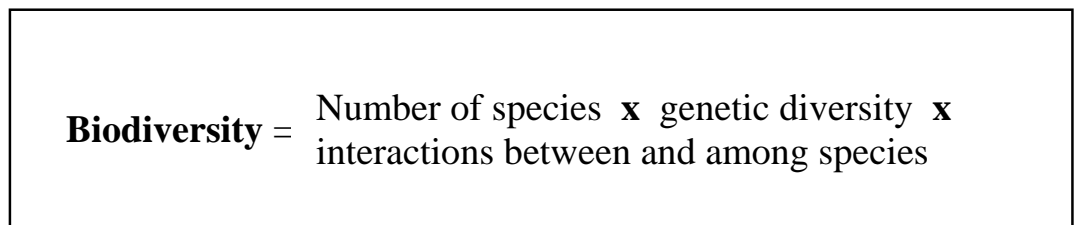

Box 2.1. A definition of biodiversity (after Kerski \& Ross, 2005, p.19).

The definition that the CBD is based on builds upon the same fundament. Thus, the CBD defines biodiversity as follows:

“[...] the variety among living organisms from all sources including, inter alia, terrestrial, marine, and other aquatic ecosystems and the ecological complexes of which they are part; this includes diversity within species, between species and of ecosystems"

(CBD, Article 2)

Besides the difficulty to find a common definition, the biodiversity concept suffers from more uncertainties. One of the most challenging uncertainties is the number of species on earth, or, numerical information on the extent of the phenomenon biodiversity ${ }^{2}$. The first and careful attempt to estimate the number of species lead to a number of 30 million assumed tropical arthropods alone (Erwin, 1982). Although this number has been largely criticized as being too imprecise and too high, a general consensus emerged assuming between 10 and 15 million species currently on earth (Pullin, 2002). However, these numbers are inaccurate and it is unlikely that we will gain certainty on this question in the near future. Closely connected to this deficit in definite numbers is the question of the exact extinction rate. As most of the world's species have not yet even been discovered, let alone classified, it is very difficult to describe the extent of species' disappearances. Moreover, the lack in definite numbers is not restricted to the level of species. At the ecosystems level, much research has to be done to better understand the complex and important interrelations within ecosystems. The same is true for the biodiversity level of genes: most plant and animal taxonomies will probably soon need extensive updates with the growing methods of DNA analyses. Thus, although biodiversity conservation emerged as the central challenge for sustainable development over

\footnotetext{
${ }^{2}$ Without information on the number of existing species, it is also impossible to estimate ecosystems and genetic diversity.
} 
the last decades, the concept is neither clearly defined, nor does it build it upon a strong data basis.

\section{$2.2 \quad$ Biodiversity hotspots}

The loss of biodiversity is a global problem. However, some regions in the world are more affected than others, for several reasons. Firstly, the diversity of species, ecosystems and genes is not distributed equally throughout the world. Some regions show extremely high biodiversity, such as tropical rainforests or other native forest ecosystems, while others show medium or low biodiversity. As a general rule, biodiversity increases from the poles to the tropics, although there is no consensus among scientists why this is so (Pullin, 2002). Due to extremely high, and at the same time "threatened" biodiversity in some of these regions, socalled biodiversity hotspots have been defined in the world (Mittermeier et al., 2004; Myers et al., 2000). The first scientific investigation aiming at a definition of hotspots led to the identification of 25 such regions (Myers et al., 2000). Two criteria had to be met to classify a region as a hotspot: i) it had to contain at least 1500 vascular plants as endemics ${ }^{3}$ and ii) it had to have $30 \%$ or less of its original vegetation. However, due to missing data, it was difficult in some cases to clearly classify a region as a hotspot (Myers et al., 2000). Four years later, with new data available, the list of biodiversity hotspots has been expanded to 34 (Mittermeier et al., 2004) (see figure 2.1).

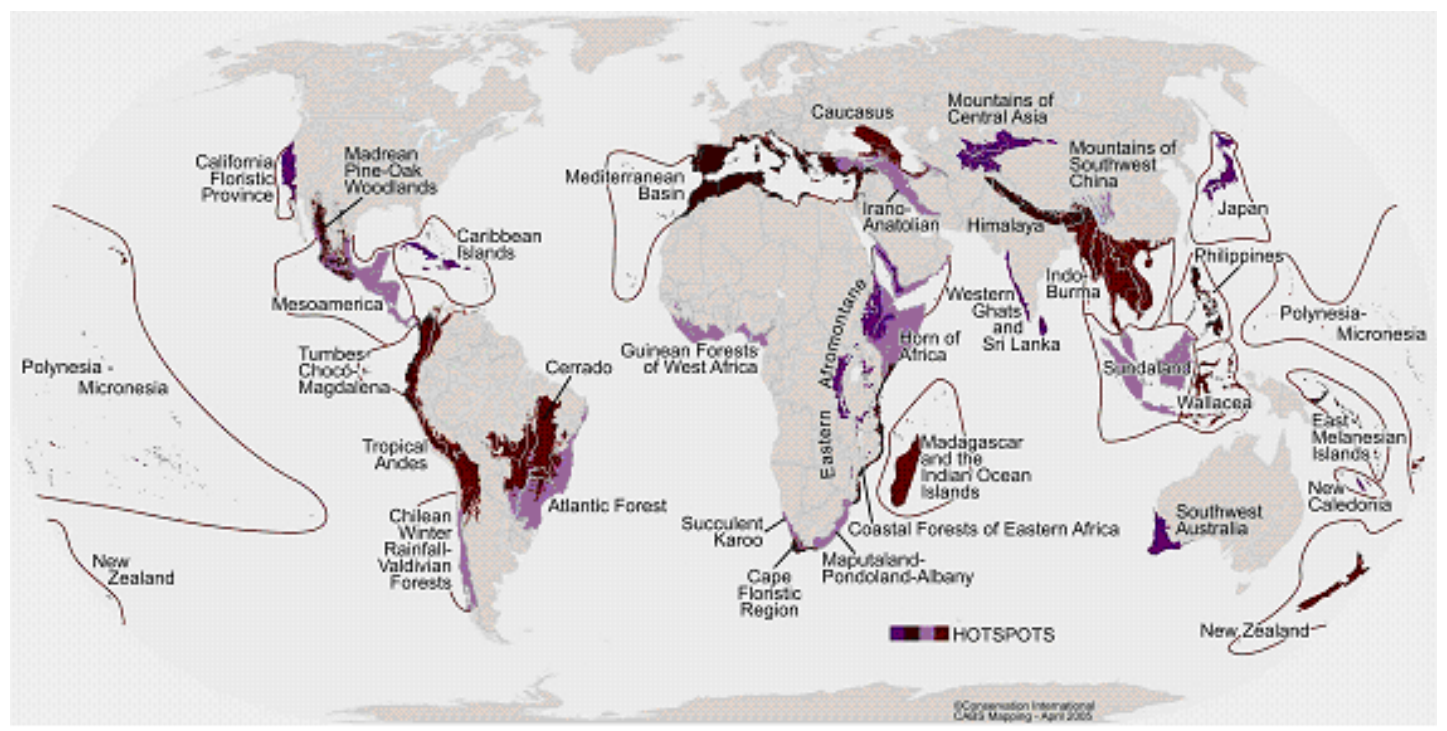

Fig. 2.1. The 34 biodiversity hotspots in the world.

(C) Conservation International 2005

\footnotetext{
${ }^{3}$ Endemism is defined as the level of species that occur naturally only in a specific region or site. Thus, an endemic species only exists in one particular area in the world.
} 
Due to their extreme species and ecosystem richness, while also having high extinction rates, biodiversity hotspots are the highest conservation priority. At the same time, conservational efforts can have the highest effect at biodiversity hotspots because a relatively high percentage of endemic and endangered species can be protected within a certain area. Therefore, certain investments have the highest conservational effect (Mittermeier et al., 2004).

A second reason for some regions being more affected than other is that current biodiversity loss is virtually completely due to human activity (Wilson, 2001). Much of this human activity is closely connected to economic interest. Biodiversity, especially biological resources, is therefore particularly under threat when poverty and high human population density coincide with each other (Cincotta et al., 2000). In fact, most biodiversity hotspots are located in so-called "developing countries". In many cases, it is the overuse of natural resources which leads to the decrease of a region's biodiversity. The overuse of natural resources is partly due to economic pressure on the individual living at a biodiversity hotspot, but also to consumption patterns of societies in the "North" (Mittermeier et al., 2004).

\subsection{Resource dilemmas}

A common resource is usually an open-access resource which is not under private ownership. In many cases, multiple parties have a certain interest in economic profit from such a common resource. However, overuse can degrade the resource and can eventually destroy it. In his classic article "The Tragedy of the Commons", Hardin (1968) described the overuse of natural resources from an economic perspective. The author presents the economic rationality behind the individual action in overuse of a common resource, which is the goal to maximize individual profit. In such a commons dilemma ${ }^{4}$, the benefits from the use of the common resource are going completely to the individual, while the negative consequences of potential overuse have to be carried by all "users". In contrast, an individual who decides to preserve the resource faces losses to his or her own personal profit. Moreover, the individual could leave the resource to be possibly depleted by other users and could therefore not be sure if his or her aim to reduce pressure on the respective plant or animal would really be achieved. Examples of resource dilemmas are fishery in common fishing grounds, or logging in forests that are not in private ownership. Ernst (1997) described psychological traps that foster such overuse of resources; namely the time trap, the

\footnotetext{
${ }^{4}$ The terms commons dilemma and resource dilemma are identical in meaning.
} 
spatial trap, and the social trap. The time trap refers to the fact that consequences of overuse of a common resource usually occur in the future. For an individual, therefore, the actual impact of his or her intervention is difficult to estimate. The time trap might also prevent an individual from judging - or even noticing - the consequences that his or her behaviour might have on the life of future generations.

The spatial trap is similar in its structure: here again, people other than the actual user of the resource have to bear possible negative consequences of overuse. An example is the pollution of rivers. Pollution in upper reaches of a river has serious consequences for those using the river downstream. Thus, those who profit from the degradation are not the ones who suffer from the consequences. The spatial trap can certainly also be of global scope. For example, “Northern” consumption patterns might seriously effect ecosystems in developing countries. Here again, the ones who profit are not those who have to bear the consequences.

The social trap focuses on the asymmetrical distribution of profit and consequences. Although degradation and consequences occur at the same time and place, people using the resource, and those suffering from the consequences, are not necessarily the same. In many cases, socially disadvantaged people are those who carry the consequences for the ecologically incorrect behaviour of others. Ernst (1997) describes these traps as serious obstacles in sustaining common natural resources. Firstly, the traps lead to a high complexity and, thus, a difficulty for the individual to see the consequences of his or her behaviour. Secondly, the motivation for an individual to preserve a resource might be low because little individual profit emerges from the preservation of the common resource, which is possibly not even within the scope of the individual's life.

\subsection{Education as a mandatory precondition for biodiversity conservation}

Biodiversity loss of the $21^{\text {st }}$ century is mainly caused by human activity. Therefore, a change in human behaviour is an important step to reduce the ongoing extinction of biodiversity on the levels of genes, species, and ecosystems. One possibility to force changes in human behaviour is through policies and laws, including penalties when unaccepted behaviour is repeatedly shown. However, in a democratic society, citizens should be able to understand and bear responsibility for regulations that the state imposes. Therefore, public information and awareness play a central role in biodiversity protection. Article 13 of the CBD explicitly takes this central role of education and public awareness into account: 
"The Contracting Parties shall:

(a) Promote and encourage understanding of the importance of, and the measures required for, the conservation of biological diversity, as well as its propagation through media, and the inclusion of these topics in educational programmes; and

(b) cooperate, as appropriate, with other states and international organizations in developing educational and public awareness programmes, with respect to conservation and sustainable use of biological diversity.”

(CBD 1992, Article 13)

In this respect, schools play a central role. According to the outcomes of the project "Defining and Selecting Key Competencies” (DeSeCo; Rychen \& Salganik, 2001), schools should impart key competencies that allow pupils to live a responsible life within a democratic society and face the challenges of the present and the future (Goody, 2001). As outlined in the previous chapters, biodiversity is clearly one of these challenges and should therefore, be addressed within the school context. Most countries that signed the CBD are now reacting to this demand by including biodiversity in their science curricula. However, for evidence based teaching practice, some important deficits emerge to which empirical science education research might contribute.

- Firstly, little is known about young peoples' ideas of biodiversity. Being a relatively new and complex concept, including global and socio-scientific issues, little research has been conducted regarding how pupils perceive biodiversity and biodiversity loss - or if they have heard of it at all.

- Secondly, little information is available on psychological factors that either positively or negatively influence young peoples' commitment to protect biodiversity. Pupils' values, beliefs, norms and the overall perception of biodiversity and its loss are important learning prerequisites that might influence both the overall understanding, and a commitment to do something about biodiversity loss.

- Thirdly, no empirical study that explicitly addresses the issue of biodiversity education has taken an intercultural perspective into account as yet. However, international cooperation with respect to biodiversity education, is explicitly encouraged by the CBD 
(CBD, 1992, Art. 13b). Pupils' perspectives on the exploitation of natural resources might differ, especially when considering resource dilemmas, depending on where they live. Therefore, an investigation into pupils' learning prerequisites at a biodiversity hotspot and an industrial country would be enlightening.

The research project "learning prerequisites for biodiversity" aims at addressing these deficits in research. Therefore, the dissertation project has two foci. Empirical responses to the first two questions are given in research focus I and research focus II of the dissertation. For the two foci, different theoretical and methodological approaches were chosen. In contrast, the third deficit in research is included as an underlying interest that affects both research foci. Thus, for both foci, an intercultural comparison between pupils in the industrial country, Germany, and in the emerging economy, Chile, was applied. In addition, the comparison covers perspectives from pupils at a biodiversity hotspot (Chile) and a country with biodiversity of medium amplitude (Germany).

Chile was chosen for the study for several reasons. Chile is a country with a rapidly emerging economy. During the last few decades, economic development has achieved high priority in domestic politics. However, this focus on economic development often implicated - and still implicates - serious consequences for the natural environment and especially for biodiversity (Figueroa \& Simonetti, 2003). Besides environmental degradation, social injustice in the form of growing disparity in income between the socially disadvantaged and the affluent is a major challenge for Chile's domestic politics today ${ }^{5}$. However, the Agenda 21 processes, including educational measures to foster sustainable development are only slowly gaining ground in practice. As a consequence, school education for sustainable development hardly exists in Chile to date (Rieckmann, 2004). Thus, on the one hand, Chilean pupils might be informed to a lesser degree about biodiversity than German pupils. However, on the other hand, it can be assumed that Chilean pupils show better understanding of biodiversity loss - especially with regard to resource dilemmas due to the presence of such dilemmas in their country. Thus, Chilean pupils' understanding of the problem of biodiversity loss might be based on personal life experiences. As a consequence, Chilean pupils might show higher commitment to protect biodiversity.

The sample for both research foci consisted of pupils in secondary education. The ages of the pupils ranged from 15-18 years old in both countries. German pupils visited the $11^{\text {th }}$ grade of

\footnotetext{
${ }^{5}$ According to the main problems Chile faces, Michelle Bachelet, since February 2006 Chile's first woman socialist Prime Minister, announced social justice and environmental protection as two of the main goals for her presidency (for more information see www.gobiernodechile.cl, accessed at April, $7^{\text {th }}$ 2007)
} 
comprehensive schools from different regions in Germany. Chilean pupils involved in the study went to the "tercero medio" respectively. This means that all the pupils were enrolled in higher education. This sampling strategy led to a selection of pupils of a particular age group and pupils who have access to secondary education. However, three specific considerations led to choosing secondary level pupils. Firstly, with biodiversity being an interdisciplinary topic, as well as one of high factual and ethical complexity (Barkmann \& Bögeholz, 2003), it made sense to first explore pupils’ cognitive frameworks at a higher age class and with pupils who showed relatively high educational success. Secondly, the questionnaire that was applied within the quantitative part of the study took, on average, 45 minutes to be completed. This means that the pupils within our sample had to be capable to concentrate specifically on a formerly unfamiliar subject matter for a relatively long time span. We assumed that, in this respect, less problems would occur the higher the age and educational level of the chosen pupils. A third reason for secondary school pupils to be chosen for the sample was the fact that we wanted to assess learning preconditions. Thus, within the sample, we decided to include pupils who had not recently come across the topic of ecology within the school context. In the chosen schools in Germany and in Chile respectively, ecology is treated in detail within the eighth grade and then again in the twelfth grade. Therefore, $11^{\text {th }}$-grade pupils represent a group that best reflects the status of learning prerequisites for the topic biodiversity on a complex, secondary school level. For detailed information on the samples, see chapters 7.6 and 9.3.

\section{$3 \quad$ Research Foci}

In the following, overall questions, theoretical backgrounds and hypotheses of the two research foci will be presented. Within research focus I, general ideas of pupils in Chile and Germany with regard to a) biodiversity and b) resource dilemmas were explored. Research focus II concentrated on commitment of pupils to actively contribute to the protection of biodiversity. In order to adequately address the two complementary research foci, different theoretical approaches were chosen that will be briefly summarized. However, for both research foci, intercultural comparisons between young people from Chile and Germany were taken into account. 


\subsection{Cognitive frameworks as learning prerequisites for biodiversity: research focus I}

\section{Overall question}

Which cognitive frameworks relevant for the topic biodiversity, biodiversity loss and resource dilemmas can be identified in Chilean and German adolescents?

\section{Constructivism and cognitive frameworks}

Learners are not blank pages when we confront them with a new topic. Every pupil has his or her own life experience upon which he or she unconsciously decides how new information will be processed. The theory of constructivism has been looking at learning processes under the focus of a person's subjective experiences for more than three decades now (cf. Carey, 1985; Caravita \& Halldén, 1994; von Glasersfeld, 2005; Piaget, 1974). Within the theory of constructivism, the main assumptions are built upon the idea that learners are active constructors of their knowledge. By doing so, learners build cognitive frameworks based on their individual experiences from early childhood. Cognitive frameworks emerge when experiences are repeatedly made. In the tradition of constructivism, there are two possibilities to process new information once a cognitive framework has been constructed. The first is when the new information can be transformed to fit into the already-existing frame of reference. This process of transforming information in order to be able to interpret it on the basis of an existing framework, is defined as assimilation. However, when the newly discovered information does not fit into the learner's framework (by being too different or by contradicting the learner's framework), or when the contradicting information is repeatedly provided, the learner can accommodate his or her cognitive framework (Carey, 1985; Hynd, McWorther, Phares \& Suttles, 1994; Posner, Strike, Hewson \& Gertzog, 1982). By doing so, the framework is transformed and is then different in structure. However, the more stable an already-existing framework is, the more likely will the newly acquired knowledge be transformed to fit into the cognitive structure (Driver \& Erickson, 1983). Although a critical discussion emerged on early (and especially radical) constructivism ${ }^{6}$, a moderate form of constructivism can be helpful to understand how a basic understanding of a new topic emerges in the learner (Duit \& Treagust, 2003; Gerstenmeier \& Mandl, 1995).

The above-outlined theoretical assumptions were applied to many kinds of learning contexts. For science learning, the role of cognitive frameworks has been intensively investigated. For

\footnotetext{
${ }^{6}$ For a detailed discussion see von Glasersfeld (2005), Devitt (1984)
} 
instance, Posner and colleagues (Posner et al., 1982) identified conceptual change processes in pupils during science learning. Hynd et al. (1994) looked at the role of instructural variables in science learning from a constructivist perspective. Georghiades (2000) described primary pupils' difficulties in transferring newly acquired scientific conceptions onto new contexts on the background of conceptual change learning. Vosniadou (1992, 1994) described how robust cognitive frameworks could be obstacles in science learning when they are opposed to a new topic. Even when newly acquired knowledge apparently seemed to be understood, and instructors therefore assumed that the learner's cognitive framework accommodated, pupils showed a strong tendency to fall back on their previously constructed frameworks. In such cases, no real accommodation took place and learning topics cannot be integrated into the learner's cognitive framework. In such cases, learning topics are subjectively interpreted, in accordance with existing patterns of thought.

To explore cognitive frameworks, it is helpful to distinguish between them according to their complexity. Groeben, Wahl, Schlee \& Scheele (1988) suggest such a classification that reflects the frameworks' complexity - and, thus, the stability within the human mind. Here, cognitive frameworks are classified as associations, concepts and subjective theories (see fig. 3.1).

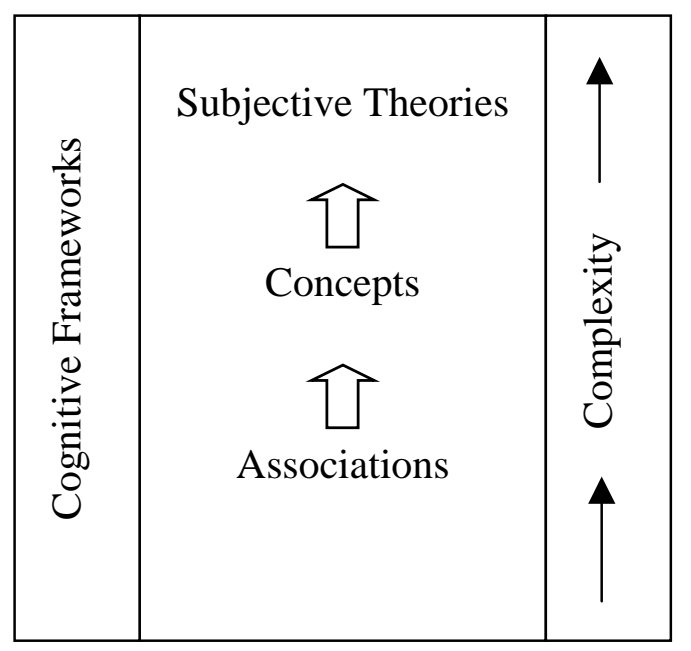

Fig. 3.1. The structure of cognitive frameworks after Groeben et al. (1988).

This dissertation follows Groeben et al.'s classification of cognitive frameworks, although an empirical differentiation of the levels is not always possible. Associations, defined as spontaneously manifested links between terms and broad ideas (Strube, 1984), can give precious hints on what pupils have casually or subconsciously perceived about a topic. Subjective theories, on the other hand, are stable cognitive structures that influence cognitive 
frameworks on less complex levels. Therefore, subjective theories are, in the context of this study, the most interesting construct to look at. Assuming that subjective theories are similar to scientific theories and that they serve as structuring elements for the interpretation of newly acquired knowledge, they might be central influential factors for the understanding of ecological, economic, and social aspects of resources dilemmas - which are in turn main drivers for biodiversity loss. For a more detailed presentation of Groeben's classification of cognitive frameworks see chapters 6.3 and 7.3.

\section{Cognitive frameworks on biodiversity}

In order to explore pupils' cognitive frameworks on biodiversity, it is helpful to follow the classification as suggested by Groeben et al. (1988). Being an exploratory approach, we tried to give room to the pupils' thoughts and ideas on the topic of biodiversity as much as possible. Therefore, in-depth, problem-centered interviews (Witzel, 2000) were chosen as a methodological approach. However, within research focus I, no concrete expectations could be formulated as too little information was provided by previous studies. Most empirical research that focuses on young people and biodiversity, explores pupils' knowledge of local plant or animal species (cf. Balmford, Clegg, Coulsen \&Taylor, 2002; Bebbington, 2005; Jäkel \& Schaer, 2004). A second group of empirical works suggests and evaluates teaching interventions about biodiversity, often focusing on taxonomic knowledge (cf. Berndt, 2000; Krombass \& Harms, 2006). Lindemann-Matthies (2002, 2006) also takes the ecosystem level of biodiversity and psychological perspectives into account. She found that her educational intervention "Species along the school path" positively contributed to both taxonomic knowledge and an appreciation of biodiversity. Moreover, she found that teachers and pupils enjoyed this kind of nature observation. However, no research findings have been provided which investigate ideas on all three levels of biodiversity. Nevertheless, a consideration of biodiversity on all three levels has been described as necessary for biodiversity education (Kassas, 2002; Mayer, 1996).

Furthermore, biodiversity should not only be considered on its three levels, but also in an interdisciplinary perspective. As described in chapter I.1, biodiversity loss is closely connected to the concept of sustainable development. With resource dilemmas as main drivers for biodiversity loss, economic, ecological and social perspectives have to be taken simultaneously into account to adequately understand this challenge for sustainable development. In science education research, a broad range of literature exists to discuss the importance to include socio-scientific issues in science education (cf. Bright \& Tarrant, 2002; Eagan, Cook \& Joeres 2002; Fien, 2002; Flint, McCarter \& Boniwell, 2000; 
Herremanns \& Reid, 2002; Kyburz-Graber, 2006; Wals \& Jickling, 2002). In this regard, biodiversity is seen as a topic that has high potential as biodiversity loss - and especially resource dilemmas! - perfectly represent such socio-scientific issues (Dreyfus et al., 1999; Ratcliffe \& Grace, 2003; van Weelie, 2002; Young, 2001). In opposition to this potential, Gayford (2000) describes difficulties of British teachers to include social aspects of biodiversity loss in their science classes. However, little is known about how pupils perceive biodiversity within the context of socio-scientific issues.

Within research focus I, the outlined research deficits in science education shall be addressed. To achieve this goal, pupils' cognitive frameworks concerning a) the three levels of biodiversity and b) resource dilemmas, respresenting socio-scientific issues in the context of biodiversity, were explored. With regard to the two samples, it was an additional focus of interest whether certain types of pupils could be identified, showing similar or differing argumentative structures when talking about biodiversity, biodiversity loss and socioscientific issues, reflected through resource dilemmas.

\section{Research questions}

The following overall research questions ( $\mathbf{Q} 1.1$ - $\mathbf{Q}$ 1.3) stood in the centre of the qualitative research focus of the dissertation.

Q 1.1 Which associations and concepts can be identified with young people from Chile and Germany that either foster or oppose an understanding of biodiversity and resource dilemmas?

Q 1.2 Can types be identified across both samples that reflect prominent subjective theories that are opposed or fostering for an understanding of biodiversity and biodiversity loss?

Q 1.3 How do cognitive frameworks differ between Chilean and German pupils?

The overall research questions will be mainly addressed in chapters four to seven. Chapters four and five present first central results. However, chapters six and seven present data that is the result of deeper analysis. Here, cognitive frameworks were, whenever possible, assigned to different levels of complexity. Furthermore, a typology was designed that reflects prominent subjective theories which emerged from the data. 


\subsection{Factors influencing pupils' commitment to protect biodiversity: research focus II}

Overall question

What factors influence Chilean and German pupils' commitment to protect biodiversity?

The Value-Belief-Norm theory

The Value-Belief-Norm (VBN) theory assumes predictors, i.e. values, beliefs, and personal norms, to explain environmentally friendly behaviour (Stern, Dietz, Kalof \& Guagnano, 1995a; Stern, Dietz, Abel, Guagnano \& Kalof, 1999; Stern, 2000). Values, personal norms and parts of the predictor block "beliefs" are based on the Norm-Activation-Model after Schwartz (Schwartz, 1977; Schwartz \& Howard, 1981). Therefore, the Norm-ActivationModel will be briefly sketched before the VBN theory will be described.

Originally, Schwartz (1977) developed the Norm-Activation-Model to explain helping behaviour, thus, altruistic behaviour. As a central point, Schwartz postulates that altruistic behaviour directly depends on an activation of personal norms. As such, personal norms are defined as "the self expectations for specific action in particular situations that are constructed by the individual” (Schwartz, 1977, p. 227). In being closely connected with a person's self-expectations, personal norms differ from social norms in that "sanctions attached to personal norms are tied to the self-concept. Anticipation of or actual conformity to a self-expectation results in pride [...]; violation or its anticipation produce guilt [...]" (Schwartz 1977, p. 231). However, a person's self-expectations - and therefore his or her personal norms - in turn depend on internalised values. Values are basic structures, which are, in contrast to personal norms, independent from specific situations. Instead, basic values are universal, although individuals might certainly have different value priorities. The NormActivation Model builds upon the assumption that values and personal norms are linked horizontally so that they "jointly influence the evaluation of the anticipated action" (Schwartz, 1977, p. 232). It follows that values as the more distal predictors influence personal norms, which in turn leads to altruistic behaviour. However, the influence of values on personal norms depends on more factors. Schwartz assumes that activation of personal norms is moderated by two factors, namely Awareness of Consequences for others (AC) and Ascription of Responsibility (AR) to prevent these consequences for others (for a detailed discussion see Schwartz \& Howard, 1981). 


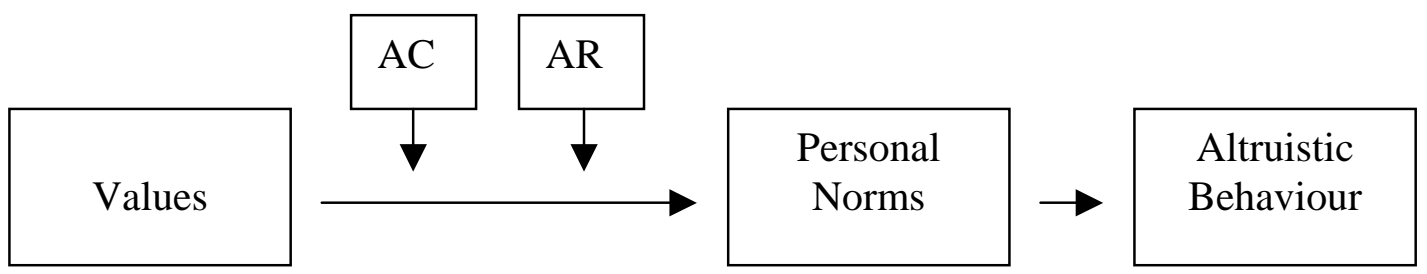

Fig. 3.2. A schematic representation of Schwartz' Norm-Activation-Model of altruistic behaviour (Schwartz, 1977; Schwartz \& Howard, 1981) AC = Awareness of Consequences; AR = Ascription of Responsibility.

As mentioned above, the Norm-Activation-Model initially aimed at explaining altruistic behaviour. However, the Model has also been successfully applied to predict proenvironmental behaviour (Black, Stern \& Elsworth, 1985; Guagnano, Dietz \& Stern, 1994; Hopper \& Nielsen, 1991; Oom do Valle, Rebelo, Reis \& Menezes, 2005; Schultz \& Zelezny, 1999; Stern, Dietz \& Kalof, 1993; Widegren, 1998). The transmission of the model to environmental contexts builds upon the assumption that altruistic and pro-environmental behaviour are motivated by the same basic values. This means that people who show a general tendency to act for the welfare of others, will also tend to act for the welfare of the environment (Hopper \& Nielsen, 1991; Stern et al., 1993).

However, Stern et al. (1993) expanded the Norm-Activation-Model to explain proenvironmental behaviour. Firstly, the value basis that formerly assumed altruistic values as positive predictors for altruism was expanded by two value orientations, the egoistic and the biospheric values. The positive influence of an egoistic value orientation was deduced from economic theory. In economic theory, human behaviour is often explained through egoistic motifs, such as in the case of resource dilemmas (Hardin, 1968; see chapter 2.3). In contrast, the assumption of biospheric values as predictors for pro-environmental behaviour derives from central ideas of the “deep ecology” movement (cf. Devall, 2001; Naess, 1997) According to the philosophy of deep ecology, nature is regarded as the valued object and a person would, therefore, develop a strong personal norm due to his or her strong value orientation towards the welfare of nature.

Stern and colleagues further expanded the Norm-Activation-Model. Besides an integration of egoistic and biospheric value orientations, the New Ecological Paradigm (NEP) ${ }^{8}$ (Dunlap,

\footnotetext{
${ }^{7}$ Deep Ecology: A form of environmentalism that advocates radical measures to protect the natural environment regardless of their effect on the welfare of people (Naess, 1997).

8 The original 12-Item-version of the NEP Scale was published 1978 under the name "New Environmental Paradigm” (Dunlap \& van Liere 1978). A "Revised NEP Scale”, now labeled “The New Ecological Paradigm” was published in 2000 as a slightly altered 15-Item-version (Dunlap et al. 2000). However, "NEP" stands for either form of the scale, original and revised as both scales aim at
} 
van Liere, Mertig \& Jones, 2000) was included as a predictor (Stern, Dietz \& Guagnano, 1995b). The NEP is considered as a measure of a general "folk ecology" (Stern et al., 1995b). This means that the NEP serves as a measurement of a general positive attitude towards nature. This has widely been used to assess a general environmentally friendly attitude (cf. Blaikie, 1992; Dunlap, 2002; Olsen, Lodwick \& Dunlap, 1992; Schultz, Unipan \& Gamba, 2000).

As an additional specification towards the Norm-Activation-Model, Awareness of Consequences (AC; as suggested by Schwartz, 1977) was included separately as perceived consequences for three valued objects: a) the self, b) the biosphere, and c) other people. This threefold structure of Awareness of Consequences is complementary to egoistic, biospheric, and altruistic value types as suggested by Stern and his colleagues (Stern et al., 1993; Stern et al., 1995a, 1995b; Stern et al., 1999; Stern, 2000). The moderator “AR” (Ascription of Responsibility) within the Norm-Activation-Model was also included in the VBN theory. AR describes the responsibility that people ascribe to themselves in protecting nature. However, the construct also covers elements of a perceived ability to reduce threats, which is important for environmental issues (Stern et al.; 1995a). In contrast to their role within Norm-Activation-Model, both the three different types of an awareness of consequences as well as ascription of responsibility, are predictors in the VBN theory, not moderators.

In summary, the VBN theory explicitly predicts pro-environmental behaviour, instead of altruistic behaviour. Four pro-environmental behavioural types serve as dependent variables of the theory, all of which explicitly reflect environmental behaviours. These behavioural types are a) activism, b) non-activist, public-sphere behaviour, c) private sphere behaviour and d) behaviour in organisations ${ }^{9}$. For examples of the respective behavioural types, see chapters 8 and 9 and appendix B. Within the VBN theory, predictors are presented in a causal ordering, moving from relatively stable elements of the personality (such as basic human values) to more focused beliefs of human-environment relations (such as the NEP, AC, and AR). According to the Norm-Activation-Model, personal norms create a general predisposition that has a strong impact on all kinds of pro-environmental behaviour (Stern, 2000).

measuring the same psychological construct. Within the empirical part of this dissertation, the revised NEP scale was used (Dunlap et al. 2000). For details see chapters 8 and 9.

${ }^{9}$ Within research focus II, all four behavioural types were assessed. However, in chapters 8 and 9, behaviour in organizations was not included in the analysis. Therefore, results on this fourth behavioural type will not be reported within this dissertation. 


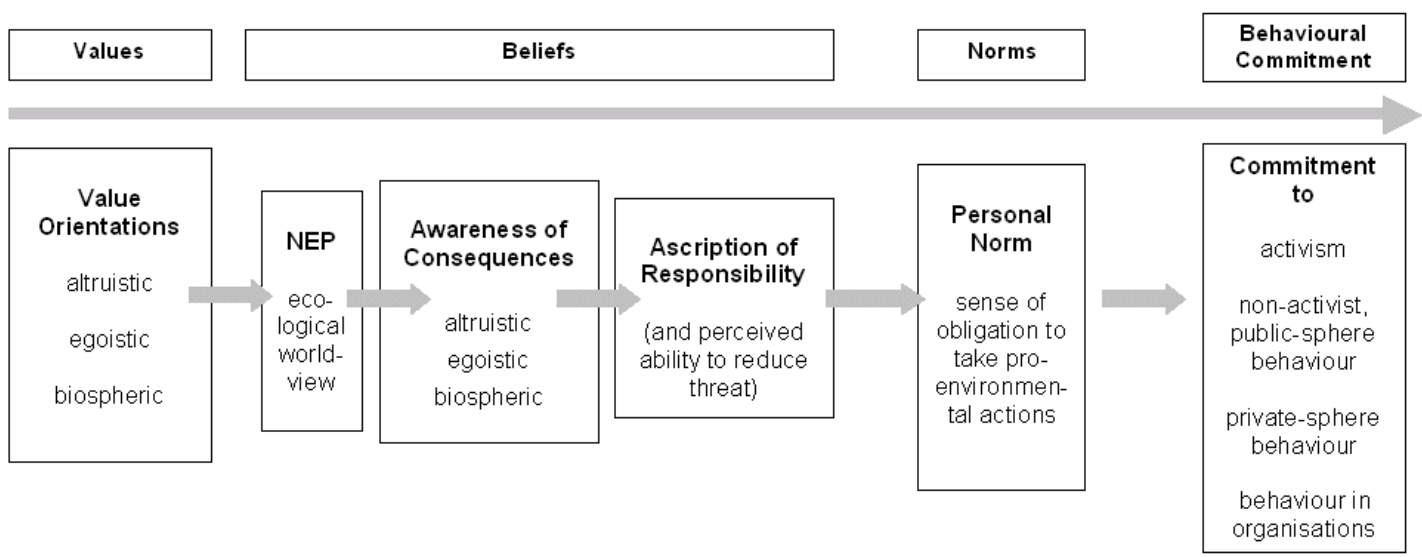

Fig. 3.3. A schematic model of the Value-Belief-Norm (VBN) theory (after Stern, 2000) $\mathrm{NEP}=$ New Ecological Paradigm.

\section{Commitment to protect biodiversity}

To the author's knowledge, the VBN theory has to date neither been applied to the context of biodiversity, nor to a sample of young people. However, in other environmentally relevant contexts, the VBN theory's contention that personal norms directly influence different types of pro-environmental behaviour has been supported (Stern et al., 1999; Widegren, 1998). Moreover, an overall set of predictors from the VBN theory accounted for between 19 and 35 percent of variance of four measured behavioural indicators (Stern et al., 1999). In an intercultural context, elements of the VBN theory have proven to be reliable predictors for a willingness to sacrifice for the environment, which in turn predicted three forms of environmentally significant behaviour (Oreg \& Tatz-Gerro, 2006). Regarding the four different behavioural commitments, they seem to be reasonable to be included in a study with a sample of young people. For instance, young people usually participate less in political activities than adults. As a consequence, in a sample with young people, differences between different behavioural options might be more crucial than in a sample with adults. Therefore, in this dissertation, three out of the four behavioural commitments as suggested in the VBN theory are included.

Besides the entire theory, elements of the VBN theory have already been tested in intercultural contexts and in the context of young people. For instance, the New Environmental Paradigm has been applied to Latin American samples (cf. Bechtel, Corral Verdugo \& Queiroz Pinhero, 1999; Kalof, Dietz, Guagnano \& Stern, 2002; Noe \& Snow, 1990; Schultz \& Zelezny, 1998; Schultz \& Zelezny, 1999; Schultz et al., 2000), and with German adolescents (Bogner \& Wiseman, 2002). Some authors successfully applied the revised NEP scale, thus, the New Ecological Paradigm, in an intercultural context (cf. Johnson, Bowker \& Cordell, 2004; Vikan, Camino, Biaggio \& Nordvik, 2007). Regarding 
the basic values, which form the basic structure of the VBN theory, it has been suggested to measure them as universal human values which have been postulated to be universal across cultural contexts (Schwartz, 1992, 1994). Schwartz developed an instrument to measure those values especially with young people (The Portrait Value Questionnaire, PVQ; Schwartz, 2005), which was an advantage for this study. Thus, the VBN theory (and elements of it) has already been supported empirically and seems to serve as a useful basis to explain pro-environmental behaviour.

The qualitative interview study within research focus I gave hints on differences in the cognitive structure of the interviewees. Within the interviews, it seemed that some of the respondents were more open towards social compounds of the problem of biodiversity loss, or, the consequences of the environmental problem for other people. However, other pupils within the study were not able to show such empathy (for details, see chapters 6 and 7). The VBN constructs "awareness of consequences" seem to address these differences and were therefore considered a suitable theoretical basis for research focus II.

The VBN theory takes, on the one hand, relatively stable psychological factors into account, such as value orientations; while on the other hand, more focused beliefs, such as an ascription of responsibility, as well as a direct commitment to act. Due to this theoretical structure, we saw high potential to integrate a set of predictors that derived from the findings of research focus I. For a detailed presentation of these additional predictors, see chapter 8.3.

\section{Hypothesis and research questions}

Besides a number of detailed hypotheses, as will be presented in chapters 8.6 and 9.2, overall hypotheses existed for research focus II. The hypotheses were partly based on the findings of research focus I and partly retrieved from already existing literature. Thus, the following overall hypotheses were formulated, which will be addressed mainly in chapters 8 and 9:

H 2.1 Chilean and German pupils differ in their personal norms and commitments to protect biodiversity through three different behavioural commitments namely, activism, non activist, public-sphere behaviour and private-sphere behaviour.

Q 2.1 Which predictors are relevant to explain Chilean and German pupils' commitments to protect biodiversity? How well do the regression models explain the three different commitments in the two samples to protect biodiversity? 
Thereafter, the empirical work will be presented in part II of the dissertation. Each of the chapters will have a different focus. Therefore, research questions will be presented in greater detail compared to the overall questions and the hypotheses that were posed within this introductory part. However, within a third part of this dissertation (III.), overall questions and hypotheses will be addressed again in the form of a summary and discussion. 
II. Empirical Part 



\section{Empirical Part}

In this second part of the dissertation, six sets of empirical questions will be addressed in chapters four to nine. Each of the presented empirical work has its own focus and will henceforth be briefly outlined.

Chapter four sketches the overall aim and the design of the combined qualitativequantitative study. Furthermore, first results of the interview study with parts of the German sample $(n=6)$ are presented. This focuses on pupils' general ideas of the terms "biodiversity" and "biological diversity", distribution and loss of biodiversity. Although the results were deduced from a small partial sample only, two concepts emerged clearly from the data when pupils explained reasons for the loss of biodiversity. Firstly, a lack of space was described as a reason for biodiversity loss. Apparently, the concept consisted of the image of a humandominated world, without space for nature. Secondly, unfavourable ecological circumstances, such rough climate or high humidity, were interpreted as harmful to biodiversity. Interestingly, both concepts were identified later as being prominent within the full sample of German pupils ( $\mathrm{n}=12$ ) (chapter 6 ) and also in the mixed sample of pupils from Chile and Germany $(\mathrm{n}=24)$ respectively (chapter 7). Chapter four closes with a brief discussion of the results and provides an outlook on the subsequent foci of the research project "Learning Prerequisites for Biodiversity".

Chapter five gives an introductory summary of how didactics of biology can contribute to promote interdisciplinary teaching and learning. Thereto, three main streams within research in didactics of biology are outlined, namely; the philosophical focus aiming at curriculum development; the focus of the practical task of teaching development and strategies; and the empirical focus, whose results provide a basis for “evidence based teaching” (Roth, 1962). This latter tradition within the didactics of biology, where this dissertation could be assigned, is then exemplarily presented in chapter five. Therefore, exemplary results were taken from research focus I, the qualitative part. The results were based on in-depth interviews with $(n=24)$ Chilean and German pupils. As a central result, the first typology of four different types was identified. However, the types were based on very general and broad argumentation structures only, which emerged from the first analysis of the data. However, in order to demonstrate how empirical results can be used for teaching practice, consequences of teaching and learning about biodiversity are discussed. 
Chapter six reports an in-depth analysis of argumentation structures within a sample of twelve German pupils. In contrast to the afore-mentioned empirical work of chapters four and five, the pupils' cognitive frameworks were investigated here in greater detail. An inductive and a deductive analysis of the interview material led to the assignment of arguments to cognitive frameworks on different levels of complexity, namely, associations, cognitions, concepts, and subjective theories. As the most complex level of cognitive structures, two subjective theories emerged from the data: one that includes and one that excludes social aspects of biodiversity loss. Since both subjective theories were prominent within the German sample, the authors created a typology on the basis of the two subjective theories. As a central result, it became evident that only parts of the pupils within the sample were able to integrate social aspects of biodiversity loss in their arguments. Moreover, those pupils who focussed exclusively on ecological aspects of biodiversity loss, were not able to show empathy for people in resource dilemmas. Therefore, in teaching practice, teachers should be aware of the potential that parts of the target audience might have to struggle to fully understand biodiversity loss, due to their existing and potentially robust cognitive structures that focus on ecological aspects of biodiversity loss.

Chapter seven follows a similar methodological approach in that cognitive frameworks were explored and then assigned to the levels of associations, cognitions, concepts, and subjective theories. However, in this case the study was based on an intercultural sample that consisted of German ( $n=12)$ and Chilean ( $n=12)$ pupils. Since results from a previous study based on the German sample already existed (chapter six), the researchers conducted no inductive analysis. As a central result, three subjective theories emerged from the data, which in turn served as the basis for a consequent typological analysis. Two of these subjective theories showed a strong disciplinary concentration of the pupils' arguments on either ecological or economic facets of biodiversity loss. However, within the third subjective theory - the only type in which social arguments were continuously applied - a balance between social, ecological, and economic arguments was achieved. The results showed that, regarding biodiversity, an integration of the three spheres of sustainable development, which is a requirement for the preservation of biodiversity, is difficult for most of pupils who were interviewed. Moreover, for Chilean pupils, it seemed to be especially challenging to recognize a local example of a resource dilemma which was demonstrated to the interviewees during the interview. In contrast, the recognition of resource dilemmas seemed to be easier for most German pupils interviewed. Nevertheless, Chilean pupils mentioned many local examples of threatened species, while only one German pupil mentioned one local example of threatened species at all. 
Chapter eight is the first chapter that represents research focus II - the quantitative part of the dissertation that aims at explaining commitment to protect biodiversity. Based on the VBN theory (e.g. Stern, 2000), the commitment of German pupils ( $n=217)$ to protect biodiversity through three different behavioural commitments was explored in a questionnaire study. In addition to the theoretical constructs based on the VBN Theory, scales for ecological and socio-economic problem perception, knowledge, and problem denial were integrated. These additional scales were representative of central argumentation structures that were identified within the qualitative focus of the study (research focus I, chapters four to seven). Overall, the VBN Theory proved to be suitable to explain young peoples' commitment to protect biodiversity, while the additional constructs were less relevant to explain the tested types of behavioural commitment. Comprehensively, our model explained between $46 \%$ and 55\% of variance for the three different types of behavioural commitment that were tested in the study. The chapter closes with a discussion of educational implications that were drawn from the structure of relevant predictors of the study.

Chapter nine reports a research focus that follows a similar theoretical and methodological approach. Here, however, predictors stemmed exclusively from the VBN theory. Additionally, the comparison between the German $(n=217)$ and the Chilean $(n=216)$ sample was a research interest. The comparison showed that Chilean pupils showed a generally higher commitment to protect biodiversity through activism, non-activist, public sphere behaviour and private sphere behaviour. Furthermore, different values and beliefs were relevant to explain the commitment to the three assessed behavioural types in the two samples. However, personal norms proved to be highly relevant for all assessed behavioural types in both samples.

As in the above outlined chapters, chapter nine closes with a discussion of the results under the perspective of biodiversity education. Additionally, a discussion regarding the consideration of the different cultural and natural environments is included. 



\section{Lernvoraussetzungen für Biodiversity Education in Deutschland und Chile am Beispiel endemischer Medizinalpflanzen ${ }^{1}$}

\subsection{Einleitung}

Der immer raschere Rückgang der Biodiversität ist ein Phänomen, dessen man sich besonders seit den 1990er Jahren zunehmend bewusst wird. Vor allem die Verabschiedung der Convention on Biological Diversity (CBD, 1992) ist ein Meilenstein, mit dessen Hilfe der Artenverlust über einen internationalen politischen Beschluss ins öffentliche Bewusstsein gerückt wurde. Artikel 13 der CBD fokussiert auf die Aufklärung und Bewusstseinsbildung in der Öffentlichkeit. Der damit verbundene Bildungsauftrag birgt für die Vertragsparteien unterschiedliche Herausforderungen. Chile und Deutschland haben das Übereinkommen über die biologische Vielfalt ratifiziert. In beiden Ländern muss jedoch von höchst unterschiedlichen Lernvoraussetzungen ausgegangen werden: Während Deutschland sich lediglich durch eine mittlere Biodiversität auszeichnet, ist Chile Biodiversity Hotspot. Insbesondere die strukturschwache VII. Region Chiles, Maule, ist als ein typischer Hotspot zu bezeichnen. Die Region weist eine hohe Diversität von Nutzpflanzen und wild wachsenden, endemischen Pflanzen auf. Letztere werden zum Teil als wildgesammelte Medizinalpflanzen vermarktet. Es handelt sich dabei um klassische RessourcenNutzungsdilemmata. Diese ökologisch-sozialen Dilemmata (Ernst, 1997) sind häufig Schlüsselphänomene beim fortschreitenden Verlust von Biodiversität. Sie können nur verstanden und gelöst werden, wenn - der CBD entsprechend - ökologische, ökonomische und soziale Aspekte gleichermaßen berücksichtigt werden.

\subsection{Biodiversity Education - eine Herausforderung für die Biologiedidaktik}

Im Bereich der Biodiversity Education im deutschsprachigen Raum benennt Mayer bereits 1996 Biodiversität als Zukunftsdisziplin in der Biologiedidaktik (Mayer, 1996). Weiter sind Veröffentlichungen von Lindemann-Matthies (2002) zu erwähnen, die sich mit konkreten

\footnotetext{
${ }^{1}$ Source: Menzel, S., \& Bögeholz, S. (2005). Lernvoraussetzungen für Biodiversity Education in Deutschland und Chile am Beispiel endemischer Medizinalpflanzen. In H. Korn, \& U. Feit (Eds.), Treffpunkt Biologische Vielfalt V (pp. 97-103). Bonn, Bad Godesberg: BfN.
} 
Interventionen zur Sensibilisierung befassen. Berndt (2000) weist auf unzureichende Unterrichtsmaterialien zur Biodiversität hin. Multimediale Informationssysteme zum Thema Biodiversität werden von Krombaß, Urhahne \& Harms und Berndt angeboten, bzw. beforscht (Berndt, 2000; Krombaß et al., 2003).

Das Zusammenspiel der Faktoren, die für den zunehmenden Biodiversitätsverlust verantwortlich sind, ist komplex und vielfältig. Biodiversity Education steht vor der Aufgabe, Ökologie, Ökonomie und Soziales gleichermaßen einzubeziehen, um adäquate Lösungsansätze für die drängendsten Probleme unserer Zeit zu ermöglichen. Die Fähigkeit zum systematischen Umgang mit faktisch und ethisch komplexen Gestaltungsaufgaben Nachhaltiger Entwicklung, wie sie die Erhaltung biologischer Vielfalt darstellt, ist eine notwendige Ergänzung zum Ziel der Verhaltensänderung klassischer Umweltbildung (Barkmann \& Bögeholz, 2003). Klassische, instrumentelle Umweltbildung ist nach Gayford “...now considered to be an out-moded idea [...] not in line with the aims of a rationale education [...]” (Gayford, 2000). Dabei geht es um anspruchsvolle Gestaltungsaufgaben Nachhaltiger Entwicklung jenseits der Umweltbildung für alltägliches Umwelthandeln im frühen Kindesalter (z. B. Vorschule und Primarstufe). Dreyfus et al. beziehen sich direkt auf Biodiversität und halten das Thema als für den Schulunterricht besonders attraktiv: Schülerinnen und Schüler werden mit einer realen Problemstellung konfrontiert, bei der auch mehr als eine Lösung geeignet sein kann. Verschiedene, teils konfligierende Weltbilder müssen in Verbindung gebracht und die eigenen sowie die Werte anderer reflektiert und diskutiert werden (Barkmann \& Bögeholz, 2003; Dreyfus et al., 1999). Besonderes Potenzial innerhalb der Biodiversitätsdiskussion bieten hier Ressourcen-Nutzungskonflikte. In der vorliegenden Studie werden die südafrikanische Teufelskralle (Harpagophytum procumbens) und Boldo (Peumus boldos) ${ }^{2}$ als Modellorganismen für endemische Medizinalpflanzen in Hinblick auf das Verstehen und Lösen ökologisch-sozialer Dilemmata untersucht.

Forschungsdefizite bestehen vor allem im Bereich der Lernvoraussetzungen zu Biodiversität, deren Gefährdung, ökologisch-sozialen Dilemmata und deren Überwindung, um Biodiversität zu erhalten. Erst wenn die Vorstellungen von Schülerinnen und Schülern bekannt sind, können Bildungsinterventionen gezielt und fruchtbar an dem Verständnis der Schülerinnen und Schüler ansetzen.

2 Boldo (Peumus boldos) ist eine in Zentralchile heimische, endemische Medizinalpflanze. 


\subsection{Untersuchungsaufbau und Methoden}

Die Untersuchung zu Lernvoraussetzungen von Schülerinnen und Schülern in Deutschland und Chile setzt sich aus einer qualitativen, explorativen (I. Phase) und einer sich anschließenden quantitativen Studie (II. Phase) zusammen. Beide Phasen werden in Chile und Deutschland durchgeführt (Tab. 4.1).

Tab. 4.1. Erhebungen von Lernvoraussetzungen von Schülerinnen und Schülern.

\begin{tabular}{|c|c|c|c|}
\hline \multicolumn{2}{|c|}{$\begin{array}{c}\text { I. PHASE: } \\
\text { QUALITATIVE, EXPLORATIVE } \\
\text { STUDIE }\end{array}$} & \multicolumn{2}{c|}{$\begin{array}{c}\text { II. PHASE: } \\
\text { QUANTITATIVE STUDIE }\end{array}$} \\
\hline $\begin{array}{c}\text { Vorerhebung } \\
\text { Interviews }\end{array}$ & $\begin{array}{c}\text { 1. Erhebungsrunde } \\
\text { Interviews }\end{array}$ & $\begin{array}{c}\text { 2. Erhebungsrunde } \\
\text { Vorstudie }\end{array}$ & $\begin{array}{c}\text { F. Erhebungsrunde } \\
\text { Hauptstudie }\end{array}$ \\
\hline $\begin{array}{c}\text { Deutschland } \\
\text { (Mai/Juni 2004) }\end{array}$ & $\begin{array}{c}\text { Deutschland } \\
\text { (Juli 2004) }\end{array}$ & $\begin{array}{c}\text { Deutschland } \\
\text { (März/ April 2005) }\end{array}$ & $\begin{array}{c}\text { Deutschland } \\
\text { (Dez. 2005/ Jan. } \\
\text { 2006) }\end{array}$ \\
\hline & $\begin{array}{c}\text { Problemzentrierte } \\
\text { Chile }\end{array}$ & $\begin{array}{c}\text { Chile } \\
\text { (November 2004) }\end{array}$ & (Mail Juni 2005) \\
(März - Mai 2006)
\end{tabular}

Die explorative Studie (I. Phase) gliedert sich in eine Vorerhebung mit explorativen Interviews und eine 1. qualitative Erhebungsrunde. Die II. Phase umfasst eine 2. und 3. Erhebungsrunde mit Fragebogenerprobung in einer Vorstudie und FragebogenHauptuntersuchung. Die Erkenntnisse der qualitativen Studie dienen unter anderem dazu, Hypothesen für die quantitative Studie zu generieren und geeignete Items zu entwickeln. Die deutsche Stichprobe setzt sich in den Erhebungsrunden 1-3 aus Oberstufenschülerinnen und schülern der 11. Jahrgangsstufe zusammen, die das Thema Ökologie im Biologieunterricht noch nicht behandelt haben. Für Chile werden die Probandinnen und Probanden im Hinblick auf Alter und Schulstufe parallelisiert. Für die explorative Studie wurden zunächst in Deutschland acht thematisch breit angelegte, teilstrukturierte, leitfadengestützte Interviews mit Schülerinnen und Schülern geführt, die im Durchschnitt zwei Stunden dauerten (Vorerhebung, siehe Tab. 4.1). Erhoben wurden Schülervorstellungen zu den Aspekten Begrifflichkeit, geographische Verteilung, Evolution, mit Biodiversität verbundene Werte, 
ökonomischer Nutzen, Gefährdungsfaktoren und Erhaltung (Helmers et al., 2004). Im Anschluss an die thematisch breit angelegte Vorerhebung wurde ein Leitfaden in Anlehnung an die Methode des „problemzentrierten Interviews“ entwickelt (Witzel, 2000). Problemzentrierte Interviews (PZI) erlauben es, neben dem Einsatz des Leitfadens vertiefende Fragen zu stellen und die Kommunikation bereits während der Befragung immer präziser auf das Forschungsproblem zuzuspitzen (Witzel, 2000). Im engeren Sinne fungiert der Interviewleitfaden im Rahmen des PZI lediglich als Rahmen im Hintergrund. Abweichend von der Methode erfolgt hier jedoch während der Interviews eine relativ enge Orientierung an denen im Interviewleitfaden vorformulierten Fragen, um die Vergleichbarkeit zwischen den Einzelinterviews zu erhöhen. Klassischerweise werden im Rahmen von PZI der Bezug auf das Subjekt wie auch auf gesellschaftliche Rahmenbedingungen, in denen Akteure handeln, hergestellt (Schmidt-Grunert, 1999). In unserem Fall besteht die problematisierte Rahmenbedingung in der Schädigung von biologischer Vielfalt durch die globale Vermarktung von Medizinalpflanzen. Die Interviews stellen den Begriff der biologischen Vielfalt, Vorstellungen über ihren Wert, ihre Gefährdung und Möglichkeiten zu ihrer Erhaltung in das Zentrum. Dabei wird auf die Rekonstruktion ökologisch-sozialer Dilemmasituationen durch Schülerinnen und Schüler und deren Lösung fokussiert. Forschungsinteressen und Beispielinterventionen sind in Tab. 4.2 aufgeführt.

Tab. 4.2. Forschungsinteresse der explorativen Studie mit Beispielinterventionen.

\begin{tabular}{|c|c|}
\hline Forschungsinteresse & Beispielinterventionen \\
\hline $\begin{array}{l}\text { Was verbinden Schülerinnen } \\
\text { und Schüler mit den Begriffen } \\
\text { biologische Vielfalt und } \\
\text { Biodiversität? }\end{array}$ & $\begin{array}{l}\text { - Was verbindest Du mit „biologischer Vielfalt“? Und } \\
\text { mit dem Begriff „Biodiversität“? } \\
\text { - Welches Bild hast Du von Orten mit hoher } \\
\text { biologischer Vielfalt? }\end{array}$ \\
\hline $\begin{array}{l}\text { Welchen Wert hat biologische } \\
\text { Vielfalt in den Vorstellungen } \\
\text { von Schülerinnen und } \\
\text { Schülern? }\end{array}$ & $\begin{array}{l}\text { - Aus welchen Gründen könnte biologische Vielfalt } \\
\text { wertvoll sein? } \\
\text { - Für wen und aus welchen Gründen kann biologische } \\
\text { Vielfalt von Nutzen sein? }\end{array}$ \\
\hline $\begin{array}{l}\text { Welche Gefährdungsgründe } \\
\text { sehen Schülerinnen und } \\
\text { Schüler? }\end{array}$ & $\begin{array}{l}\text { - An welchen Stellen auf der Erde ist eine Bewahrung } \\
\text { der biologischen Vielfalt wohl am schwierigsten? } \\
\text { - Worin liegen die Ursachen? } \\
\text { - Stelle dir die Produkte vor, die du im täglichen Leben } \\
\text { verwendest. Was könnten diese Produkte mit } \\
\text { biologischer Vielfalt zu tun haben? }\end{array}$ \\
\hline
\end{tabular}


Tab. 4.2 (Fortsetzung)

\begin{tabular}{|c|c|}
\hline Forschungsinteresse & Beispielinterventionen \\
\hline $\begin{array}{l}\text { Werden ökologisch-soziale } \\
\text { Dilemmata erkannt? }\end{array}$ & $\begin{array}{l}\text { Die Teufelskralle, die nur im Süden Afrikas wächst, gilt } \\
\text { als im Bestand gefährdet. } \\
\text { - Woran kann das liegen? } \\
\text { - Welches Interesse haben Menschen daran, Pflanzen } \\
\text { zu ernten und zu verkaufen, obwohl die } \\
\text { entsprechende Art bedroht ist? } \\
\text { Boldo-Blätter stammen aus Wildsammlung. } \\
\text { - Könntest du dir vorstellen, welche Probleme durch } \\
\text { die Gewinnung, Nutzung und Vermarktung von } \\
\text { Boldo entstehen? } \\
\text { - Kennst Du weitere Beispiele, in denen der Handel und } \\
\text { Verkauf von Arten diese in ihrem Bestand bedroht } \\
\text { haben? }\end{array}$ \\
\hline $\begin{array}{l}\text { Welche Lösungsansätze sehen } \\
\text { Schülerinnen und Schüler? }\end{array}$ & - Welche Lösungen könnte es für die Probleme geben? \\
\hline
\end{tabular}

Die Stichprobe der 1. Erhebungsrunde umfasst 12 Schülerinnen und Schüler von drei nordrhein-westfälischen Schulen (ein dörfliches bis kleinstädtisches Gymnasium, ein kleinstädtisches Gymnasium, eine Großstadt-Gesamtschule). Durch Erhebungen an drei Schulen sollen mögliche Verzerrungen durch Lernerfahrungen (spezielle Unterrichtseinheiten, hoch engagierte Lehrpersonen) sowie regionalspezifische Erfahrungen relativiert werden. Die 12 Einzelinterviews dauerten durchschnittlich 30-45 Minuten. Sie wurden mittels Diktiergerät aufgezeichnet, transkribiert und anschließend zu redigierten Aussagen zusammengefasst.

Der vorliegende Beitrag berichtet über erste Auswertungen von 6 der 12 geführten Interviews. Die Auswertung erfolgt computergestützt durch das Programm MAXqda (Version 2001). Dargestellt werden Ergebnisse der Analysen zum Verständnis der Begrifflichkeit, zu Wertzuschreibungen und zu Gefährdungsgründen von biologischer Vielfalt. Auswertungskategorien wurden in Anlehnung an Miles \& Huberman (1994) generiert.

\subsection{Erste Ergebnisse}

In Abb. 4.1 sind die Antwortkategorien abgebildet, die auf Basis des Datenmaterials generiert wurden. Zunächst werden die Ergebnisse zu Vorstellungen über biologische Vielfalt, anschließend zur Biodiversität, dann zu deren Wert und Gefährdung dargestellt. 

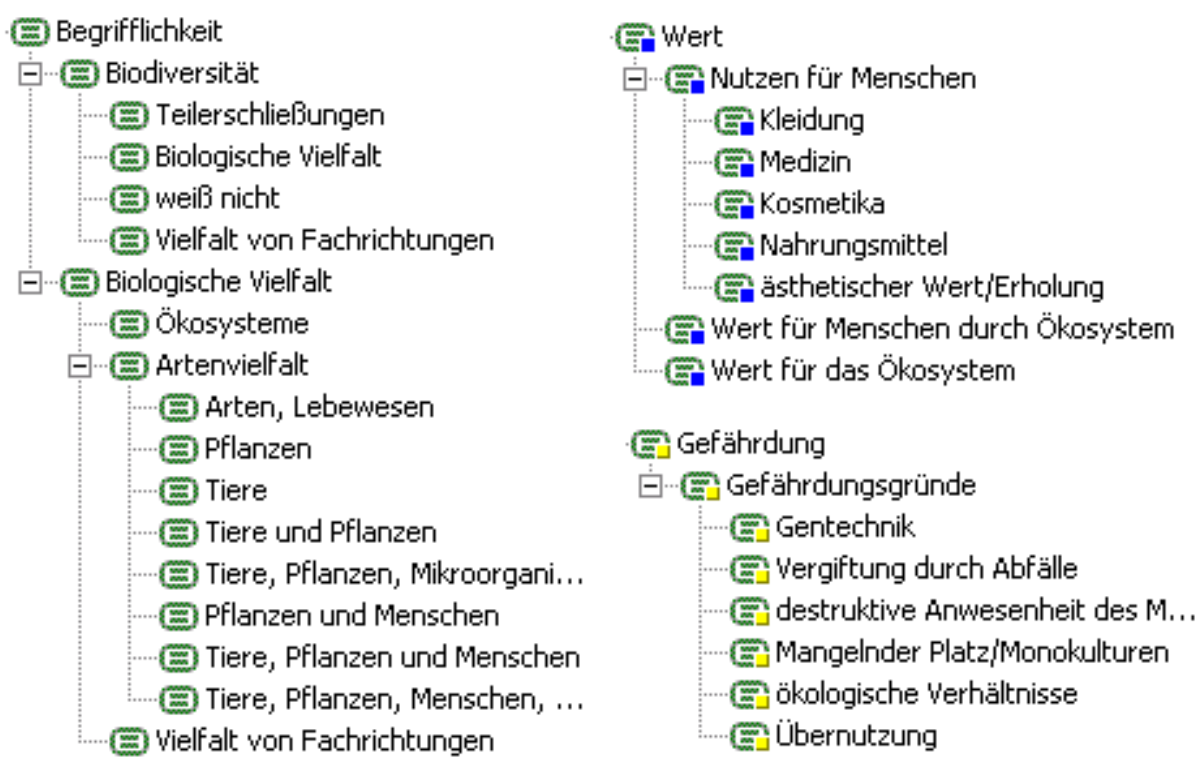

Abb. 4.1. Auswertungsschema der Kategorien Begrifflichkeit, Wert und Gefährdung der Biodiversität.

Die Auswertung der ersten 6 Interviews der Erhebungsrunde 1 (Deutschland) zeigt, dass Schülerinnen und Schüler dem Begriff „biologische Vielfalt“ mit Unsicherheit begegnen. Der Begriff wird alltagssprachlich hergeleitet und führt in den meisten Fällen zu einer Interpretation als Unterschiedlichkeit von Pflanzen und Tieren. Teilweise werden jeweils nur Tiere oder nur Pflanzen benannt, um biologische Vielfalt zu charakterisieren: „[Biologische Vielfalt in Bezug auf Natur] das sind in der Biologie vielleicht verschiedene Pflanzen oder all so etwas zum Beispiel, es gibt ja nicht nur einen Baum. Es gibt ja verschiedene Bäume, Kirschbaum und [so weiter]“ (Natascha ${ }^{3}$ 98-99 ${ }^{4}$ ) oder „[Durch] wenige oder viele, große oder kleine Tierarten [könnten Unterschiede in der biologischen Vielfalt sichtbar werden]“ (Olaf 140, 144). Widersprüchlich sind Konzepte von Schülerinnen und Schülern, welche die Rolle des Menschen betreffen. Zum Teil liegen bei einer Person sowohl Aussagen vor, die den Menschen explizit von biologischer Vielfalt ausschließen und ihn als Störfaktor interpretieren, wie auch Konzepte, die den Menschen in seiner Verschiedenheit als Teil der biologischen Vielfalt ansehen. Eine weitere vorunterrichtliche Vorstellung besteht in der Interpretation des Begriffs „biologische Vielfalt“ als „Vielfalt von Fachrichtungen innerhalb der Biologie“. Die Vorstellung erweist sich zum Teil als stabil. Obwohl eine Klärung des Begriffs vorgenommen wurde, verwendet ein Schüler im Interviewverlauf seine Vorstellung von der Vielfalt der biologischen Einzeldisziplinen weiter.

3 Die Ziffern geben die Zeilenzahl im Originaltranskript des Interviews an.

4 Alle Namen von Schülerinnen und Schülern wurden geändert, um die zugesicherte Anonymität zu gewährleisten. 
Alle befragten Schülerinnen und Schüler geben an, den Begriff „Biodiversität“ noch nie gehört zu haben; sie können sich auch nicht an eine Verwendung des Begriffs in der Schule oder durch die Medien erinnern. Der Bitte, mit dem Begriff frei zu assoziieren und nach langsamem Vorsprechen Ideen zu äußern, können nicht alle Befragten Folge leisten. Auch können Teilannäherungen an den Begriff nach dem Hinweis, sich an biologische Vielfalt zu erinnern, nur sehr fragmentarisch vorgenommen werden. Teilerschließungen sind beispielsweise: „Bio ist etwas mit Bio und [wo]von Diversität kommt, weiß ich nicht.“ (Sebastian 57-58, 62) oder „Vielleicht [meint Biodiversität] irgend etwas Gegensätzliches“ (Olaf 58).

In Bezug auf den Wert von biologischer Vielfalt äußern alle Befragten die Wichtigkeit für den Fortbestand von Ökosystemen, beispielsweise durch Aussagen wie: „[Es ist wichtig, dass es viele Arten gibt] weil, das ist auch wieder ein Ökosystem für sich. Zum Beispiel jetzt wird ein Frosch gefressen von irgend einem Tier, das wird dann wieder gefressen, das ist so ein Kreislauf“ (Anna 168-170). Der Wert der biologischen Vielfalt für den Menschen wird in den meisten Fällen erst nach entsprechender Intervention benannt. Konkrete Beispiele für den Zusammenhang zwischen biologischer Vielfalt und dem Nutzen für den Menschen kommen dann häufig aus dem Bereich der Nahrungsmittel, Kosmetik und Medizin. Beispiele werden in praktisch allen Fällen erst nach der Aufforderung genannt, den eigenen Tagesablauf unter dem Gesichtspunkt von Produkten, die etwas mit biologischer Vielfalt zu tun haben könnten, zu reflektieren.

In Bezug auf Gefährdungsfaktoren dominieren zwei Konzepte: Erstens, biologische Vielfalt ist bedroht durch mangelnden Platz („Platz-Konzept“). Entsprechend sind Beispiele raumeinnehmende Industrien, Monokulturen und Viehhaltung, auf die teilweise mehrfach zurückgegriffen wird. So äußert Anna beispielsweise auf die Frage, wo die Erhaltung der biologischen Vielfalt wohl am schwierigsten sei: „[In den Industriegebieten ist es so schwierig] weil man halt viele Maschinen baut und da nichts mehr richtig wachsen kann, wenn man alles zubetoniert“ (Anna 265-266). Als zweites dominantes Konzept werden schwierige ökologische Verhältnisse (Konzept „ungünstige ökologische Bedingungen“) für eine Abnahme der biologischen Vielfalt verantwortlich gemacht: „Schwierige Lebensbedingungen [sind eine weitere Ursache, warum die Bewahrung der biologischen Vielfalt in den Tropen besonders schwierig ist]. [Zum Beispiel] der Wechsel zwischen Sonne und den starken Regenfällen“ (Olaf 263, 267). Gefährdung biologischer Vielfalt durch Übernutzung („Übernutzungs-Konzept“) wird erst nach einer Intervention im Interview genannt, die darin bestand, den durch Vermarktung verursachten Gefährdungsstatus der Medizinalpflanzen Boldo und Teufelskralle darzustellen. Eine systematische Auswertung der Vorstellungen zu ökologisch-sozialen Dilemmata ist noch nicht erfolgt. Als erste Tendenz ist 
jedoch festzuhalten, dass Schülerinnen und Schüler zwar in der Lage sind, einzelne Akteure eines Ressourcen-Nutzungskonflikts zu benennen, jedoch in der Regel das Verhalten der verschiedenen Akteure nicht systematisch miteinander in Beziehung setzen.

\subsection{Diskussion und Ausblick}

Die Ergebnisse zu Vorstellungen über die Begriffe „biologische Vielfalt“ und „Biodiversität“ sind konsistent mit der oftmals nur als Artenvielfalt in den Richtlinien für Schulen aufgegriffenen Facette von Biodiversität. Interessant ist, dass auch nach einer Phase des Nachdenkens keine Vorstellungen über Biodiversität geäußert werden können. Der Begriff biologische Vielfalt wird alltagsweltlich hergeleitet, wobei teilweise Facetten von biologischer Vielfalt oder Fehlvorstellungen als Lernvoraussetzungen identifiziert werden können. Während die Schülerinnen und Schüler ökologische und ökonomisch-instrumentelle Werte, zumindest auf Nachfrage, mit biologischer Vielfalt verbinden, wird der absolute Eigenwert (intrinsische Wert) nicht angeführt. Betrachtet man die Konzepte im Bereich der Gefährdungsfaktoren, bei denen negative anthropogene Einflüsse lediglich durch den Verbrauch von Platz definiert werden, so ist zu vermuten, dass die weiteren überwiegend anthropogen bedingten Gefährdungsfaktoren nicht bekannt sind. Bei der Erklärung von Umweltproblemen scheint die Vorstellung eines Stadt-Land-Gefälles sehr dominierend zu sein, bei dem die „gesunde“ Landnatur verstädterten Regionen gegenübergestellt wird. Die modellhaften Beispiele, aus denen das „Platz-Konzept“ zur Bedrohung biologischer Vielfalt abgeleitet wird, sind nicht immer gleich gut geeignet, Gefährdungsgründe zu erklären. Im Laufe des Interviews wird häufig auf diese Beispiele zurückgegriffen, auch bei der Erklärung ökologisch-sozialer Dilemmasituationen. Ähnliches zeigt sich in Hinblick auf Vorstellungen über biologische Vielfalt (siehe Vorstellung „Vielfalt biologischer Einzeldisziplinen“). Es ist zu vermuten, dass Schülerinnen und Schüler vertraute Konzepte als Modelle für die Interpretation neuer Situationen verwenden. Besonderer Stellenwert kommt damit der didaktisch-methodisch reflektierten Erarbeitung möglichst geeigneter exemplarischer Modelle zu.

Neben der Einbeziehung der weiteren 6 Interviews in die Analysen $(n=12)$, werden weitere Auswertungen auf Schülervorstellungen zu ökologisch-sozialen Dilemmata fokussieren. Denkbar wäre eine Auswertung des Datenmaterials nach der Komplexität des Verständnisses von Dilemmasituationen (Rekonstruktion faktischer und ethischer Komplexität) und vorgeschlagenen Lösungsansätzen. Weiterhin wird untersucht, inwiefern Schülerinnen und Schüler rekonstruierte Strukturen ökologisch-sozialer Dilemmata am Beispiel der 
Teufelskralle (a) auf das Ressourcen-Nutzungsdilemma von Boldo übertragen können und (b) inwiefern die Strukturen die Beschreibung weiterer eigener Beispiele erlauben: Stellt doch das Verständnis ökologisch-sozialer Dilemmata, die Übertragung erkannter Dilemmastrukturen und die Befähigung zur Überwindung derartiger Dilemmata eine zentrale Herausforderung für Bildung für Nachhaltige Entwicklung und damit auch für Biodiversity Education dar. 



\section{Caminos empíricos en la investigación didácitica para mostrar la necesidad de una enseñanza transdisciplinaria sobre la biodiversidad ${ }^{1}$}

\subsection{Abstract}

The field of didactics of biology is commonly understood as a discipline that prepares biological issues to be suitable for school teaching. However, within the field of didactics of biology, different research foci exist. For example, besides the already-mentioned (and in fact quite important) field of preparation of teaching contents, the field related to philosophical aspects, such as school and curriculum development exists. Empirical research forms the third research current within the didactics of biology. In this paper, an example will be given of how empirical research results can contribute to an enhancement of teaching and learning processes in that they show how important an interdisciplinary treatment of the topic biodiversity is. The results that are presented were taken from a qualitative interview study with German ( $\mathrm{n}=12)$ and Chilean $(\mathrm{n}=12)$ pupils aged 16-18. The following research questions were addressed:

1. How do pupils reconstruct resource dilemmas?

2. Is it possible to identify a typology that is based on ecological, economic, and social arguments that the pupils used when they explained resource dilemmas?

The problem-centered interviews were fully transcribed and analyzed deductively in order to identify ecological, economic, and social arguments that pupils applied when they explained resource dilemmas. As a central result, four types could be identified within the sample: i) the ecological type, ii) the ecological-economic type, iii) the ecological-social type and iv) the social type. Within three out of the four types, pupils focussed on ecological arguments. Furthermore, it seemed that, the more pupils focussed on ecological reasons of biodiversity loss, the more false ecological contents were expressed. At the same time, a strictly disciplinary consideration of the issues lead to an incomplete understanding of biodiversity

\footnotetext{
${ }^{1}$ Source: Menzel, S. (2006). Caminos empíricos en la investigación didácitica para mostrar la necesidad de una enseñanza transdisciplinaria sobre la biodiversidad. In M. Quintanella (Ed.), Educación Científica, Ciudadanía y Valores: Retos de los Nuevos Modelos de Conocimiento (CDROM). Santiago de Chile: Pontificia Universidad Católica de Chile \& UNESCO.
} 
loss and a tendency to apply false ecological contents. Moreover, Chilean pupils had problems to identify a given local resource dilemma. Only three pupils were able to continuously consider social arguments within the three stages of the interview and therefore succeeded in identifying resource dilemmas. These results suggest that an interdisciplinary reflection of biodiversity loss and resource dilemmas is challenging, but at the same time important.

\subsection{Introducción}

Las nociones generales sobre las didácticas de las asignaturas escolares en muchos casos solo sirven como instrumento para comprimir la materia de una asignatura para alumnas y alumnos. De hecho, eso es un parte importante de la didáctica, pero no la parte exclusivo (véase figúra 5.1).

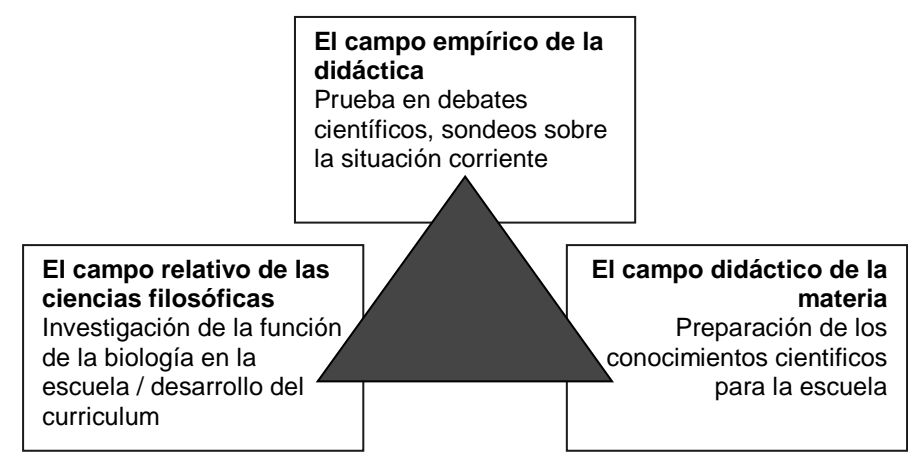

Fig. 5.1. Tres campos de la didáctica.

Aparte del campo didáctico de la materia existen los campos relativos a las ciencias filosóficas y empíricas de la didáctica. Objeto del campo filosófico es por ejemplo la decision sobre contenidos de la asignatura correspondiente, por lo tanto el desarrollo del curriculum de la escuela. El campo empírico de la didáctica entrega pruebas empíricas para conjeturas acerca de la enseñanza. Si, por ejemplo existe una sospecha que una metodología en la enseñanza podría ser más efectiva que una otra, la didáctica empírica puede relizar investigaciones sistematicas para aclarar este punto.

Una pregunta actual en la didáctica de las ciencias naturales es si una apertura de las asignaturas cientificas frente una transdisciplinaridad pueda mejorar o dañar el proceso de aprendizaje. El estudio empírico presente es un ejemplo para una investigación tratando esta pregunta. El modelo es el tema de la biodiversidad, un problema urgente y asimismo complejo que hasta ahora se ha tratando enmarcado en la asignatura de biología, predominantamente en una manera disciplinaria. 


\subsection{Biodiversidad: téma urgente y complejo}

La biodiversidad está seriamente en peligro en muchos regiones del mundo (Wilson, 1992). La diminuación de la biodiversidad no únicamente toca la extinción de especies, pero también la extinción de la diversidad genética y ecológica (Townsend, et al., 2003). La pérdida de la biodiversidad es mucho más compleja que el concepto de la extinción de especies. Sobre todo en pretendidos «Hotspots» de la biodiversidad, el problema es especialmente grave (Myers et al., 2000). Hotspots son regiones con una alta biodiversidad, las cuales tienen simultáneamente problemas sociales y economicos graves. La situación económica y social tan difícil de éstas regiones implica una presión extremamente alta sobre recursos naturales y la biodiversidad. La relación entre problemas sociales y economicos y la presión alta sobre la biodiversidad está marcada por las llamadas «crisis socio-ecologicas» (Ernst, 1997 ; Hardin 1968). Estas situaciones de crisis tienen como protagonistas a personas que deben destruir un recurso natural para sobrevivir economicamente. Un ejemplo conocido es la deforestación de bosques virgenes. Chile central es un hotspot de biodiversidad, que ha vivido varios ejemplos de crisis socio-ecologicas.

En la didáctica, las investigaciones sobre la biodiversidad se concentran mayoritariamente en la diversidad de especies (por ejemplo Jäkel \& Schaer 2004; Lindemann-Matthies, 1999). Muchas investigaciones en didáctica de la biología se interesan por los conocimientos adquiridos por los alumnos sobre las especies locales. Hasta ahora, una perspectiva mundial sobre la biodiversidad sólo ocurre en pocos proyectos de investigación de la didáctica. Tampoco existen investigaciones acerca de la complejidad del téma, que es un desafío con muchas pretensiones en la didáctica (Barkmann \& Bögeholz, 2003).

\subsection{Preguntas de investigación}

Para estudiar como los alumnos pueden aprender mejor el tema, las siguentes preguntas de investigación son el eje del proyecto presentado :

1.¿Cómo reconstruyen los alumnos crisis socio-ecologicas?

2. Es posible encontrar una tipología acerca de los argumentos ecológicos, económicos y sociales utilizados por las alumnas y alumnos? 


\subsection{Metodología}

La investigación empírica fue una investigación cualitativa con entrevistas. El grupo objetivo se concretó en un total de 24 alumnos de 16 y 17 años en Chile y Alemania. Las entrevistas se realizaron de forma semi-estructurada (Witzel, 2000) utilizando una guía de entrevista, siguiendo tres pasos (véase fig. 5.2).

En un primero paso, se hicieron preguntas generales sobre los factores que amenazan la biodiversidad. Además, en el primer paso presentamos un ejemplo sobre una planta bajo riesgo, la garra del diablo. La planta está expuesta a un comercio excesivo ya que sus raíces se emplean para la producción de medicamentos. Esta planta crece exclusivamente en la selva en el sur de África, y no es posible hacer cultivos extensivos. Aunque las poblaciones de la planta ya están gravemente disminuidas, los recolectores, mayoritariamente personas que no poseen terrenos cultivables, siguen recogiendo las raíces de la planta, fuente de compuestos bioactivos. La situación es un ejemplo clásico de una crisis socio-ecologíca. Nos basamos en este ejemplo para investigar si los alumnos tienen la capacidad de ver expontáneamente causas sociales y economicas para la perdida de un recurso natural. Durante esta primera parte de la entrevista, se ofrecieron intervenciones orales para el alumno en situaciones en las que las implicaciones sociales y economicas no fueron identificadas espontanéamente por el alumno.

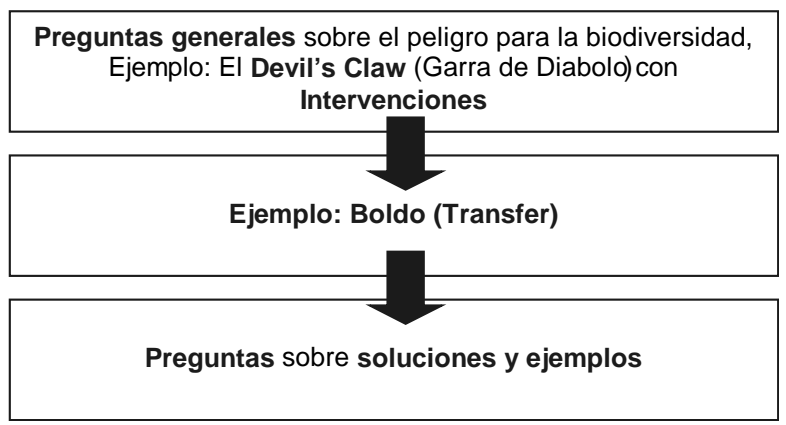

Fig. 5.2. Guía de entrevista.

El segundo paso de la entrevista fue la presentación de un segundo ejemplo de una crisis socio-económico con estructuras muy similares. El ejemplo fue una planta muy conocida en Latinoamérica, el Boldo. A diferencia del primer paso, no ofrecimos ayudas a los alumnos para que ella/el vean activamente razones sociales y economicos. 
Una tercera parte de la entrevista fue un paso con preguntas sobre posibles soluciones perceptibles por la alumna o el alumno. Un tema muy intereseante fue identificar si el alumno/a fue capaz de desarollar soluciones fuera de los elementos ecologicos del problema. Después la grabación completa de las entrevistas, se hicieron transcripciones de todas preguntas y respuestas. Para facilitar la valoración de datos, los textos fueron transformados a una forma gramaticalmente correcta. Los textos transformados fueron procesados en el programa computacional, MAXqda2 (Kuckartz, 2004), un programa especialmente desarollado para el analísis de datos cualitativos. Mediante este programa se desarrollaron categorías para ordenar las respuestas de los alumnos/as en los tres pasos de la entrevista categorizando el lado ecológico, económico y social del problema.

\subsection{Resultados}

El resultado de los análisis de argumentos empleados por los alumnos/as durante los tres pasos de la entrevista mostró cuatros tipos de alumnos. Cada típo está caracterizado por una tendencia en la estructura de sus argumentos específicos. Los tipos identificados son:
1. El típo ecológico
2. El típo ecológico-económico
3. El típo ecológico-social
4. El típo social "desarrollo sostenible”

A continuación nos focalizaremos en los tipos ecológico y social «desarrollo sostenible», los cuales presentan marcadas diferencias.

\section{El típo ecológico}

Los alumnos asignados de este típo utilizan predominantamente argumentos ecológicos para explicar el problema de la perdida de la biodiversidad (veáse fig. 5.3). En el paso 1 de la entrevista, cuando se hacen intervenciones para mostrar activamente al alumno implicaciones sociales y economicas, los argumentos puramente ecologicos son dominantes. 


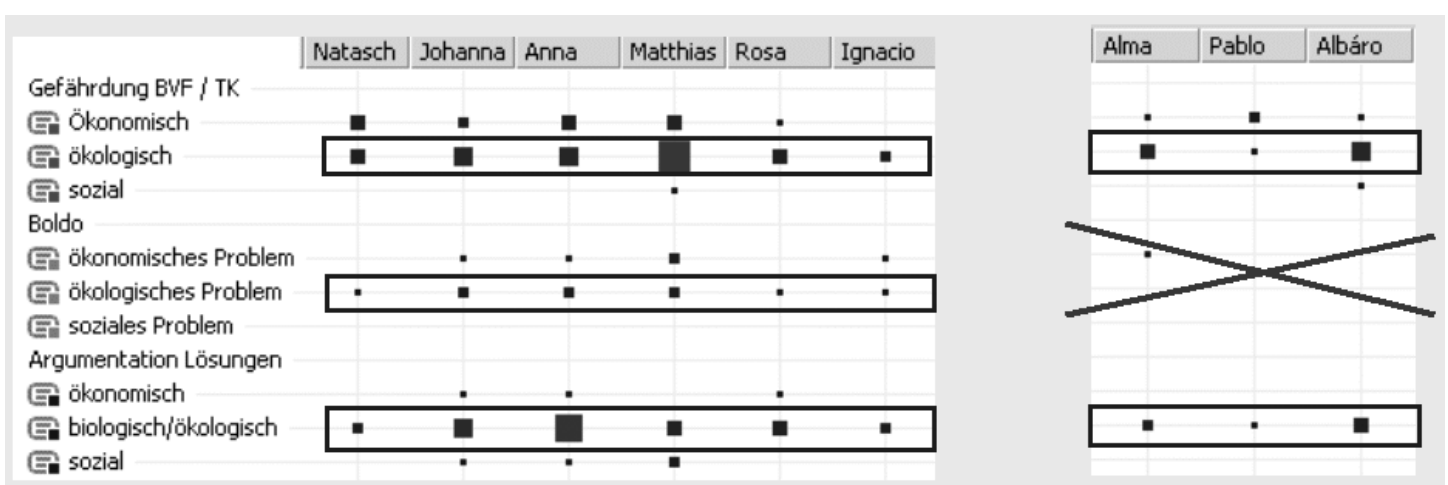

Fig. 5.3. El típo ecológico. La figura muestra los argumentos con puntos: Cuanto los puntos son más grandes, los argumentos fueron más frecuentes. Se grafican los argumentos en los tres pasos de la entrevista : Argumentos economicos (ökonomisch), ecologicos (ökologisch) y sociales (sozial) en el nivel de la perdida de la biodiversidad general (Gefährdung), en el caso del Boldo y para soluciones (Lösungen).

A lo sumo en un paso de la entrevista los argumentos ecologicos son igual fuertes que argumentos ecologicos o sociales (por ejemplo en el caso de Matthias, paso dos, Boldo). En estos casos se nota que en los dos pasos siguientes de la entrevista, los argumentos ecologicos son claramente dominantes. Un fenómeno muy interesante es que los alumnos/as chilenos, aparentemente, tienen problemas con la percepción de la estructura del ejemplo local del boldo. En estos casos, desglosamos el paso dos de la valoración. Así mismo sin argumentos en el segundo paso de la entrevista, estas alumnas y alumnos chilenos son parte del tipo ecológico. ¿Cómo describen alumnos del típo ecológico el problema? Ejemplos clásicos ofrecen una alumna alemana, Natascha y un alumno chileno, Alvaro. ${ }^{2}$

Natascha (17): [...] para las plantas, no sé. Hay algunas plantas [que] no creecen de nuevo una vez que están arruinadas o algo [parecido] (647-648) ${ }^{3}$

Quizás después de algún tiempo, [...] nada creece de nuevo. Depende del suelo o del tiempo. (723-724) [Yo llamaría al comportamiento de los participantes individuales en el caso del Boldo] egoístas. Quizás ellos tienen que pensar en el resto de la gente también.

En conclusión, si la gente continúa actuando de esta forma, no tendremos ninguna naturaleza, nada. (955-957)

Natascha trata de explicar la pérdida de la garra del diablo exclusivamente por razones ecologicas. Sus intentos de explicar el problema de forma puramente ecológica implica supuestos positivosen ese sentido, como el hecho que la supervivencia de la planta depende de factores ecologicos, come el suelo o el sol. Sin reconocer implicaciones sociales y económicas, Natascha tiene prejuicios graves sobre los recolectores de la planta. Este manera

\footnotetext{
${ }^{2}$ Todos los nombres presentados están cambiados.

${ }^{3}$ Los números en parentesis significan los números de las líneas correspondientes en los textos transformados.
} 
de interpretación es lógica para una persona que no es capaz de ver las razones fuera de la ecología para la destrución de una planta.

Alváro (17): Amenaza [la biodiversidad biológica] es que todo en grandes cantidades produce cambios, entonces puede ser si hay muchos; [...] árboles por ejemplo que destruyen el suelo, y eso, todo cuanto es mucho, es malo. $(300-301,305,309)$

[Las soluciones que puedo imaginar respecto a este problema que tiene esta planta] no sé, sacar el ADN tenerlo guardado y también plantarla en lugares donde se pueda dar mejor, hacer una investigación de la planta y guardar el ADN. (491, 495, 499, 503)

Sancionar a los que la destruyen, dando un tipo de castigo [como medida preventiva para la protección de la diversidad biológica] $(622,627)$ Yo creo que la sanción, [es la medida más efectiva] si. (645)

La estructura de argumentos de Alváro es parecido de los argumentos utilizados por Natascha: Razones y soluciones para el problema se orientan exclusivamente a la faceta ecológica. Este típo de interpretación de la pérdida de la biodiversidad tampoco permite al alumno tomar la perspectiva de los recolectores de la planta. Las soluciones a los ojos de Alváro son establecer sanciones contra las personas que extraen la planta. El alumno no considera soluciones para la situación social, ni para la situación económica de la gente involucrada.

\section{El típo social "desarrollo sostenible"}

La estructura de argumentos de los alumnos/as de este típo se diferencia claramente del típo ecológico. El criterio más importante es que los argumentos sociales son utilizados en todos los tres pasos de la entrevista (veáse fig. 5.4).

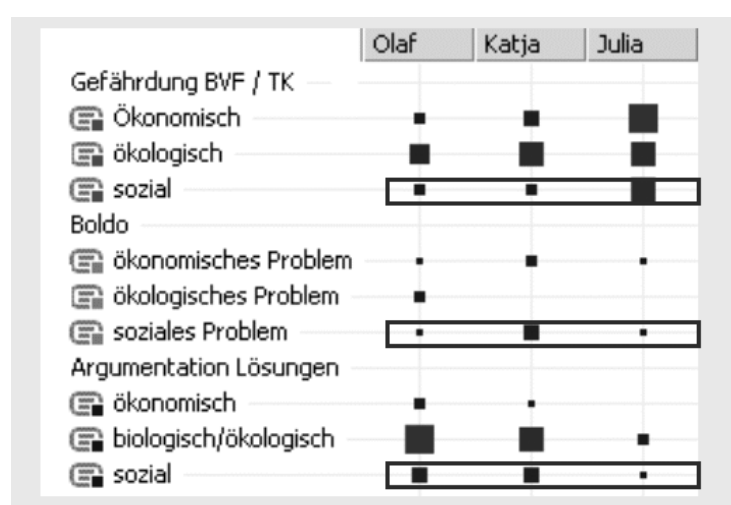

Fig. 5.4. El típo social “desarrollo sostenible”. 
En este caso, es muy manifiesto que hay una homogeneidad relativa entre argumentos ecologicos, economicos y sociales. A diferencia del típo ecológico, las alumnas y alumnos de este típo no enfocan tan fuertamente en una faceta los problemas sobre la pérdida de la biodiversidad. Un ejemplo son las declaraciones de la alumna alemana, Katja :

Katja (16): [Acerca de las recolecciones en la selva] puedo imaginarme que, cuando la [la planta] no está cultivada y se le colecta solo como así, un día todo estará perdido y las plantas ya no podrán extenderse lo suficiente. (209-212) Creo que [la situación es tan complicada por que] la gente quien saca la [la planta], sea como sea, necesita este dinero, de otra manera no tendrían caminos para sobrevivir. $Y$ quizás es que no tienen la posibilidad de hacer algo diferente y la [gente] realmente necesita el dinero [hace la situación tan difícil]. (398-401)

Katja es un ejemplo para una alumna quien quiere describir la pérdida de la biodiversidad como un problema no únicamente ecológico. Ella integra argumentos sociales y economicos y consecuentamente ve la situación de los recolectores de la planta de forma más realista. Katja percibe la situación de forma ecológica también, pero conoce el hecho de que la situación ecológica no es suficiente para explicar integramente la pérdida de una especie. Ella manifiesta que soluciones no es fácil a causa de la complejidad económica y social del problema. La reconstrucción de la situación elaborado por Katja es, entonces, más completa y no existen prejucios negativos sobre los colectores de la planta.

\section{$5.7 \quad$ Implicaciones}

Los resultados muestran que los alumnos/as de 16 y 17 años evidentemente tienen problemas con una reconstrución interdisciplinaria de la pérdida de la biodiversidad. Generalmente, los alumnos de los dos paises, Alemania y Chile, prefieren argumentos ecológicos. Solo pocos alumnos figuran en el grupo social «desarrollo sostenible», el único grupo con una estructura de argumentos más integros. Los alumnos/as chilenos evidentamente tienen problemas adicionales con la reconstrucción de la crisis socio-económica presentada durante la entrevista, en el caso del boldo. La presencia fuerte en los mercados locales podría ser un razón por la cual un riesgo de extinción para esta planta es dificil de entender para los alumnos chilenos.

Sobre todo, el estudio muestra un punto central: La inclusión de materias interdisciplinarias no perturba el proceso de aprendizaje en la Biología. Parece que el suceso contrario tiene lugar: Las alumnas y alumnos quien se concentran exclusivamente sobre relaciones ecologicas tienen la tendencia de desarrollar ideas ingenuas acerca de la ecología. 
Probablemente las personas del típo ecológico se sientan descontentas con su análisis del problema y desarrollan consecuentemente nuevas explicaciones, las cuales, en muchos casos pueden ser incorrectos. 



\section{Vorstellungen und Argumentationsstrukturen von Schüler(inne)n der elften Jahrgangsstufe zur Biodiversität, deren Gefährdung und Erhaltung ${ }^{1}$}

\subsection{Zusammenfassung und Abstract}

Für eine Bildung für Nachhaltige Entwicklung ist die Thematik Biodiversität eine komplexe Herausforderung: Beim Biodiversitätsverlust sind ökologisch-soziale Dilemmata schwierig zu durchschauende Schlüsselprobleme, die über ökologische Zusammenhänge hinaus gehen. In einer Interviewstudie wurden Vorstellungen von Schüler(inne)n der 11. Klasse zum Verständnis ökologisch-sozialer Dilemmata, zur Biodiversität, deren Verbreitung und Gefährdung auf der Ebene der Assoziationen, Kognitionen, Konzepte und subjektiver Theorien untersucht ( $\mathrm{n}=12)$. Es ist festzuhalten, dass (a) der Begriff Biodiversität wenig bekannt ist, (b) eine ausschließlich ökologische Betrachtung des Biodiversitätsverlusts mit negativen pauschalen Urteilen über Menschen in ökologisch-sozialen Dilemmata einher gehen kann und (c) die eigene Verantwortung als Konsument(in) in ökologisch-sozialen Dilemmata meist nicht reflektiert wird. Nach einer typologischen Analyse von Argumentationen bezüglich ökologisch-sozialer Dilemmata konnten drei Typen identifiziert werden: ein ökologisch orientierter, ein ökologisch-sozial orientierter und ein Retinitäts-Typ. Der Artikel fordert eine Bildung zur Biodiversität (a) mit konsequent interdisziplinärer Ausrichtung und (b) unter Verdeutlichung der eigenen Rolle der Schüler(innen) zum Schutz der Biodiversität.

\section{Abstract}

Conservation of biological diversity is a complex challenge for teaching students about sustainable development: A key threat to biodiversity are commons dilemma, which are particularly difficult to understand and by far exceed purely ecological reasons. Interviews were used to explore the cognitive frameworks of 11th-grade German students $(n=12)$ with respect to their understanding of resource dilemmas as well as their understanding of

\footnotetext{
${ }^{1}$ Source: Menzel, S., \& Bögeholz, S. (2006). Vorstellungen und Argumentationsstrukturen von Schüler(inne)n der elften Jahrgangsstufe zur Biodiversität, deren Gefährdung und Erhaltung. Zeitschrift für Didaktik der Naturwissenschaften, 12, 199-217.
} 
biodiversity, its distribution, and potential threats. The data suggests that (a) students were largely unaware of the term biodiversity; (b) that students' ecological considerations of biodiversity loss could be associated with negative global judgements about people in resource dilemmas; and (c) that students did not consider their own responsibilities as consumers within resource dilemmas. Based on a typological analysis of students' argumentations of such resource dilemmas, three different types of students were identified namely, an ecologically-oriented type, an economically-orientated type, and a sustainabilityorientated type. These findings suggest that biodiversity education should be interdisciplinary and should also include a clarification of students' own roles for protecting biodiversity.

\subsection{Biodiversität als Herausforderung für eine Bildung für Nachhaltige Entwicklung}

Die Erhaltung der Biodiversität ist eine der größten Herausforderungen des gegenwärtigen Jahrhunderts (Wilson, 1992). Bildung ist eine entscheidende Strategie, um Biodiversitätsschutz effektiv gesellschaftlich zu verankern. Es wird aufgezeigt, inwiefern das Bildungsziel „Erhaltung der Biodiversität“ international gesetzlich verankert ist und anschließend ausgeführt, wo eine Bildung zur Erhaltung der biologischen Vielfalt verortet werden kann.

\section{Internationale Festlegung von Handlungserfordernissen}

Die Biodiversität oder biologische Vielfalt ${ }^{2}$ umfasst die Ebenen der Artenvielfalt, der Gene und der Ökosysteme. Der Begriff „Biodiversität“ war im Sinne dieser Definition in der Vergangenheit zunächst rein ökologisch geprägt. Seit den späten 1980er Jahren erhielt er jedoch mit zunehmendem Bewusstsein für ökonomische und soziale Bezüge starke politische Implikationen. Den Verlust der Biodiversität erkannte man als eines der drängendsten Probleme des 21. Jahrhunderts, das nicht ausschließlich ökologisch motiviert ist (z.B. WCED, 1987; Wilson, 1992). Um den Schutz der biologischen Vielfalt auf politischer und juristischer Ebene zu verankern, wurde auf dem Umweltgipfel in Rio 1992 die Convention on Biological Diversity (CBD) verabschiedet, die der Problemstruktur des Biodiversitätsverlusts entsprechend, Ökonomie, Ökologie und Soziales gleichermaßen

2 Die beiden Begriffe sind bedeutungsgleich und können synonym verwendet werden. In der Wissenschaft ist ersterer gebräuchlicher. 
berührt. Die Bundesrepublik Deutschland hat das völkerrechtlich verbindliche Dokument ratifiziert und sich somit zur Umsetzung der in der Konvention festgelegten Strategien zur Erhaltung der Biodiversität verpflichtet. Bildung kommt beim Schutz der biologischen Vielfalt eine zentrale Rolle zu: Artikel 13a der CBD bezieht sich explizit auf den Bildungsauftrag der Vertragsstaaten (CBD, 1992, Art. 13a).

\section{Bildung für Nachhaltige Entwicklung und Bildung zur Biodiversität}

Eine Bildung für Nachhaltige Entwicklung ist eine Herausforderung für alle Unterrichtsfächer und die außerschulische Bildungsarbeit. Nur durch Bildung können politische Entscheidungen (wie die Ratifizierung und Umsetzung der CBD) im Sinne einer demokratischen Gesellschaft von ihren Mitgliedern getragen und unterstützt werden (Goody, 2001). Biologieunterricht hat eine zentrale Bedeutung bei der Umsetzung der Bildungsziele der CBD (CBD, 1992, Art. 13, Niedersächsisches Kultusministerium, 2001), da er in der Oberstufe zu einer Nutzung biologischen Wissens im Alltag und in der Gesellschaft verhelfen soll (Mayer, Harms, Hammann, Bayrhuber \& Kattmann, 2004). Bedeutsam ist hierbei, die hinter dem Biodiversitätsverlust stehenden, globalen Zusammenhänge zu erkennen und die eigene Rolle in diesem komplexen Gefüge positionieren zu können. Um dies zu bewältigen, müssen Schüler(innen) lernen, mit faktisch und ethisch komplexen Gestaltungsaufgaben wie der Erhaltung der Biodiversität umgehen zu können (Barkmann \& Bögeholz, 2003).

\subsection{Theoretischer Hintergrund, Stand der Forschung und Forschungsfragen}

Im folgenden Kapitel sollen die theoretischen Grundlagen der vorliegenden Arbeit erläutert werden. Nach der Definition der Begriffe Schülervorstellungen, Assoziationen, Kognitionen, Konzepte und Subjektiver Theorien folgt die Erläuterung ökologisch-sozialer Dilemmata, die eine Hauptursache beim Verlust der Biodiversität darstellen. Das Kapitel schließt mit dem Stand der Forschung zur Biodiversitätsbildung, dem Aufzeigen von Forschungsdefiziten und den Forschungsfragen.

\section{Schülervorstellungen}

Schüler(innen) verfügen über Vorstellungen zu Unterrichtsinhalten bevor diese im schulischen Kontext behandelt werden (Posner et al., 1982). Aus konstruktivistischer Perspektive werden Lerner(innen) als aktiv Strukturierende ihres Wissens angesehen (z.B. 
Piaget, 1974). Neue Lerninhalte werden so mit bereits vorhandenem Wissen und vorhandenen Vorstellungen verknüpft. Obwohl die radikal-konstruktivistische Theorie nicht ohne Kritik geblieben ist, kann Wissen über Schülervorstellungen dazu beitragen, diese systematisch im naturwissenschaftlichen Unterricht aufzugreifen (Duit \& Treagust, 2003). Der Begriff Schülervorstellungen wird hier zusammenfassend für kognitive Strukturen auf unterschiedlichen Komplexitätsebenen verwendet. Ihre Komplexität betreffend, können sie in aufsteigender Reihenfolge in Assoziationen, Kognitionen, Konzepte und Subjektive Theorien differenziert werden.

Treten spontane Äußerungen zu einem Themenkomplex auf, kann es sich hierbei um Assoziationen handeln. Wir folgen hier einer allgemeinen Definition als spontane Verknüpfungen von Assoziationsgliedern, in diesem Fall Begriffen (Strube, 1984). Assoziationen sind dabei keine reinen Gedächtnisleistungen und können so, moderiert durch konstruktive und strategische Prozesse, erste Hinweise auf eine komplexere mentale Struktur wie Kognitionen und Konzepte liefern.

Kognitionen sind in der Psychologie weit weniger klar definiert (Groeben et al., 1988) und nicht immer deutlich von Konzepten zu trennen. Vielmehr stellen sie nach Groeben et al. (1988) vergleichsweise einfache Phänomene wie Begriffe oder wenig komplexe Konzepte dar; oder Teilkonzepte, die untereinander nicht systematisch in Beziehung stehen.

Konzepte sind komplexere Strukturen und nach Groeben et al. (1988) als Vorstufen einer Subjektiven Theorie zu verstehen. Sie können sich wiederum aus verschiedenen Kognitionen zusammen setzen.

Subjektive Theorien sind relativ überdauernde kognitive Strukturen, die komplexe Aggregate von Konzepten darstellen und teilweise implizit sind. Sie besitzen ähnliche strukturelle Eigenschaften wie wissenschaftliche Theorien und sind unter anderem dazu da, eine Realitätskonstituierung vorzunehmen (Groeben et al., 1988). Ihnen kommt handlungsleitende und handlungssteuernde Funktion zu (Dann, 1994). Subjektive Theorien sind häufig stabil (Vosniadou, 2001), selbst wenn sie fachwissenschaftlich nicht korrekt sind und von Lernenden als nicht ausreichend erkannt werden. Um die Veränderung bzw. Erweiterung von Schülervorstellungen durch fachwissenschaftliche Konzepte zu optimieren, ist es wichtig, vorhandene Schülervorstellungen zu kennen: Schulischer Unterricht kann dann systematisch auf vorhandenen Vorstellungen der Lerner aufbauen.

\section{Ökologisch-soziale Dilemmata}

Ökologisch-soziale Dilemmata (Ernst, 1997) sind häufig Schlüsselphänomene beim Verlust von Biodiversität. Sie haben durch soziale und ökonomische Interessen einen erhöhten 
Druck auf eine natürliche Ressource zur Folge. Zudem zeichnen sie sich durch eine hohe faktische und ethische Komplexität aus (Barkmann \& Bögeholz, 2003) und können nur verstanden und gelöst werden, wenn ökologische, ökonomische und soziale Aspekte gleichermaßen berücksichtigt werden.

Ökologisch-soziale Dilemmata sind klassische Allgemeingut-Dilemmata (Hardin, 1968). Zur Übernutzung einer gemeinschaftlichen Ressource kommt es in ökologisch-sozialen Dilemmasituationen, weil alle Beteiligten, oft zur Sicherung ihrer Grundbedürfnisse, individualrational handeln und über die Ressource bis zu ihrer Erschöpfung verfügen. Im Bereich des Themas Biodiversität sind ökologisch-soziale Dilemmata für Schüler(innen) besonders schwer zu durchschauen, da deren Verständnis einen Perspektivwechsel und Empathie mit Menschen in Dilemmasituationen voraussetzt. Um ökologisch-soziale Dilemmata zu verstehen und auf globaler Ebene zu lösen, müssen außerdem ökologische, ökonomische und soziale Komponenten gleichermaßen betrachtet werden. Eine ausschließlich ökologische Betrachtung der Problematik ist nicht ausreichend, um den Verlust der biologischen Vielfalt als globales und interdisziplinäres Problem zu rekonstruieren (z.B. Shiva, 2000; Townsend et al., 2003; Wilson, 1992).

\section{Stand der Forschung zur Biodiversitätsbildung}

Dreyfus et al. (1999) bezeichnen Biodiversität als besonders geeignetes Schulthema, um mit Schüler(inne)n reale, komplexe Probleme zu bearbeiten. Gayford (2000) zeigt in seiner qualitativen Studie vor allem die Unsicherheit von Lehrer(inne)n in England bezüglich interdisziplinärer Aspekte des Themas Biodiversität auf. Salinas Hernández (2002) thematisiert die diverse Darstellung ökologischer Komponenten der Biodiversität in Schulbüchern und zeigt einen niedrigen Wissensstand andalusischer Schüler(innen) in diesem Bereich auf.

Im deutschsprachigen Raum wird Biodiversität bereits 1996 als Zukunftsdisziplin in der Biologiedidaktik benannt (Mayer, 1996). Lindemann-Matthies (2002b, 2006) zeigt in einer Evaluationsstudie, dass ein Bildungsprogramm für Grundschüler(innen) mit erkundenden und ästhetischen Naturerfahrungen die Wahrnehmung und Wertschätzung von biologischer Vielfalt fördern kann. Empirisch nachgewiesen wurde außerdem ein Wissens- und Interessenszuwachs durch ein multimediales Informationssystem zur Artenvielfalt (Krombass \& Harms, 2006). Defizite im Bereich der heimischen Artenkenntnisse von Schüler(inne)n fünfter und sechster Klassen weisen Jäkel und Schaer (2004) nach. Menzel und Bögeholz (2005) identifizieren erste Vorstellungen von Schüler(inne)n zu Biodiversität. Forschungsdefizite bestehen in Bezug auf Vorstellungen von Schüler(inne)n über 
Biodiversität, vor allem auf interdisziplinärer Ebene. Weithin unbekannt sind Vorstellungen zu ökologisch-sozialen Dilemmata, die als ein Hauptauslöser für den Verlust der Biodiversität angesehen werden können.

\section{Forschungsfragen}

Die vorliegende Studie verfolgt zwei Ziele und verbindet diese. Da über Lernvoraussetzungen zur Biodiversität wenig bekannt ist, sollen zunächst Schülervorstellungen auf ihren unterschiedlichen Komplexitätsebenen der Assoziationen, Kognitionen, Konzepte und Subjektiven Theorien identifiziert werden. Hierbei stehen sowohl Vorstellungen zur Biodiversität als auch zu ökologisch-sozialen Dilemmata im Zentrum der Betrachtungen.

Als zweites Ziel soll herausgefunden werden, ob der Struktur ökologisch-sozialer Dilemmata entsprechend ökonomische, ökologische und soziale Auslöser für den Verlust der Biodiversität gleichermaßen erkannt und in Verbindung gesetzt werden, und welche Subjektiven Theorien zu einer möglicherweise einseitigen Betrachtung führen können. Von Interesse ist hierbei auch, ob innerhalb der Stichprobe eine Typologie entwickelt werden kann, die prominente Subjektive Theorien repräsentiert.

Wir gehen daher folgenden Forschungsfragen nach:

a) Über welche Vorstellungen verfügen Oberstufenschüler(innen) in Bezug auf „biologische Vielfalt“ und „Biodiversität“, deren Wert, Verbreitung und Gefährdung?

b) Welche Subjektiven Theorien von Schüler(inne)n sind im Bereich der ökologisch-sozialen Dilemmata erkennbar?

c) Sind Typen erkennbar, die aufbauend auf Subjektiven Theorien prominente Argumentationsstrukturen verfolgen?

\subsection{Untersuchungsaufbau und Methoden}

Um Vorstellungen und Argumentationsstrukturen von Schüler(inne)n zu untersuchen, wurde eine qualitativ-explorative Interviewstudie durchgeführt. Im Folgenden wird zunächst die Zusammensetzung der Stichprobe erläutert. Im Anschluss wird die Erhebungs- sowie Auswertungsmethodik vorgestellt.

\section{Auswahl der Proband(inn)en}

Für die Interviews wurden Schüler(innen) der elften Klasse ausgewählt. Der Wissensstand 
entspricht den Voraussetzungen, von denen bei einer Bildung zur Biodiversität auf Oberstufenniveau ausgegangen werden kann ${ }^{3}$.

Interviewt wurden 12 Schüler(innen) im Alter von 17 und 18 Jahren (s. Tab. 6.1). Eine Auswahl von vier Interviewpartner(inne)n pro Schule erfolgte durch die unterrichtenden Lehrkräfte. Wir baten darum, Personen mit unterschiedlichem Leistungsniveau auszuwählen. Die Teilnahme erfolgte freiwillig.

Tab. 6.1. Zusammensetzung der Stichprobe. Die Namen aller Proband(inn)en wurden geändert.

\begin{tabular}{|c|c|c|c|}
\hline Name (geändert) & Schule & Alter & Schulisches Leistungsniveau \\
\hline Sebastian & \multirow{4}{*}{ Gymnasium Kleinstadt } & 17 & sehr gut \\
\hline Matthias & & 18 & schwach \\
\hline Katja & & 17 & mittel \\
\hline Johanna & & 17 & mittel \\
\hline Natascha & \multirow{4}{*}{ Gesamtschule Großstadt } & 18 & schwach \\
\hline Julia & & 17 & mittel \\
\hline Anna & & 17 & sehr gut \\
\hline Ayshe & & 17 & mittel \\
\hline Simone & \multirow{4}{*}{ Gymnasium auf dem Land } & 18 & sehr gut \\
\hline Lilli & & 18 & mittel \\
\hline Olaf & & 17 & schwach \\
\hline Andreas & & 17 & mittel \\
\hline
\end{tabular}

\section{Datenerhebung}

Für die Datenerhebung wurde das Problemzentrierte Interview ausgewählt (Witzel, 1989). Das Interview wurde durch einen Leitfaden strukturiert, der zugleich Interventionen innerhalb des Interviews auf einem Niveau hielt. Trotzdem erlaubt die Methode, vertiefende Fragen zu stellen und auf das im Zentrum stehende Problem ergänzend Bezug zu nehmen. Vor Beginn der Einzelinterviews wurden die Testpersonen darüber informiert, dass es sich um keine fachwissenschaftliche Überprüfung handelt, es also keine „falschen“, nur willkommene Äußerungen geben kann. Es wurde absolute Anonymität zugesichert.

3 In den meisten Lehrplänen der Bundesländer wird das Thema Ökologie in der achten Klasse behandelt, wobei Begriffe wie „Ökosystem“, „Nahrungsnetz“ und das „Prinzip der gegenseitigen Abhängigkeiten“ auf einfachem Niveau anhand heimischer Ökosysteme eingeführt werden. In der Oberstufe ist eine tiefere Behandlung des Themas vorgesehen. 


\section{Aufbau des Interview-Leitfadens}

Im Interview-Leitfaden wurden neben den zu stellenden Fragen das Forschungsinteresse sowie erwartete Schülerantworten formuliert. Auszüge aus Fragen und Forschungsinteresse des Leitfadens sind in Tabelle 6.2 dokumentiert. Der eingesetzte Interviewleitfaden besteht aus drei miteinander verknüpften Phasen (Abb. 6.1). In der ersten Phase werden allgemeine Aspekte des Themas „Biodiversität“ thematisiert, z.B. Fragen über die Begriffe „Biodiversität“ und „biologische Vielfalt“ sowie die Gefährdung und Verbreitung. Als zusätzliche Intervention werden den Befragten verschiedene Produkte aus pflanzlichen Rohstoffen präsentiert, z.B. Tees und medizinische Salben. Ziel war es zu ermitteln, ob ein spontaner, lebensweltlicher Bezug zwischen den angebotenen Produkten und biologischer Vielfalt hergestellt werden kann. Am Ende der ersten Phase steht eine sprachliche Intervention zu einer ökologisch-sozialen Dilemmasituation: Modellorganismus ist die südafrikanische Teufelskralle (Harpagophytum procumbens), deren Bestände durch vermehrten Export auf den europäischen Markt gefährdet sind. Der Leitfaden sieht für dieses erste Dilemmabeispiel mehrere Hinweise vor, die ökologische, ökonomische und soziale Sichtweisen auf das Problem ermöglichen (Tab. 6.2).

Tab. 6.2. Auszüge aus dem Interview-Leitfaden.

\begin{tabular}{|c|c|}
\hline Forschungsinteresse & Beispielinterventionen \\
\hline $\begin{array}{l}\text { Was verbinden Schüler(innen) } \\
\text { mit den Begriffen „biologische } \\
\text { Vielfalt" und „Biodiversität“? }\end{array}$ & $\begin{array}{l}\text { - Was verbindest Du mit dem Begriff „biologische Vielfalt“? Und mit } \\
\text { dem Begriff „Biodiversität“? } \\
\text { - Welches Bild hast Du von Orten mit hoher biologischer Vielfalt? }\end{array}$ \\
\hline $\begin{array}{l}\text { Welchen Wert hat biologische } \\
\text { Vielfalt in den Vorstellungen von } \\
\text { Schüler(inne)n? }\end{array}$ & $\begin{array}{l}\text { - Aus welchen Gründen könnte biologische Vielfalt wertvoll sein? } \\
\text { - Für wen und aus welchen Gründen kann biologische Vielfalt von } \\
\text { Nutzen sein? }\end{array}$ \\
\hline $\begin{array}{l}\text { Welche Gefährdungsgründe } \\
\text { sehen Schüler(innen)? }\end{array}$ & $\begin{array}{l}\text { - An welchen Stellen auf der Erde ist die Bewahrung der biologischen } \\
\text { Vielfalt wohl am schwierigsten? } \\
\text { - Worin liegen die Ursachen? } \\
\text { - Stelle Dir Produkte vor, die Du im täglichen Leben verwendest. Was } \\
\text { könnten diese Produkte mit biologischer Vielfalt zu tun haben? }\end{array}$ \\
\hline $\begin{array}{l}\text { Werden ökologisch-soziale } \\
\text { Dilemmata erkannt? }\end{array}$ & $\begin{array}{l}\text { Die Teufelskralle, die nur im Süden Afrikas wächst, gilt als im Bestand } \\
\text { gefährdet. } \\
\text { - Woran kann das liegen? } \\
\text { - Welches Interesse haben Menschen daran, Pflanzen zu ernten und } \\
\text { zu verkaufen, obwohl die entsprechende Art bedroht ist? } \\
\text { Boldo Blätter stammen aus Wildsammlung. } \\
\text { - Könntest Du Dir vorstellen, welche Probleme durch die Gewinnung, } \\
\text { Nutzung und Vermarktung von Boldo entstehen? } \\
\text { - Kennst Du weitere Beispiele, in denen der Handel und Verkauf von } \\
\text { Arten diese in ihrem Bestand bedroht haben? }\end{array}$ \\
\hline $\begin{array}{l}\text { Welche Lösungsansätze sehen } \\
\text { Schüler(innen)? }\end{array}$ & - Welche Lösungen könnte es für die Probleme geben? \\
\hline
\end{tabular}


Die dritte Phase des Interviews führt auf eine weitere Abstraktionsebene. Ohne Interventionen werden Schüler(innen) gebeten, eigene Beispiele für Biodiversitätsverlust durch ökologisch-soziale Dilemmata zu geben und Lösungsansätze zu entwickeln.

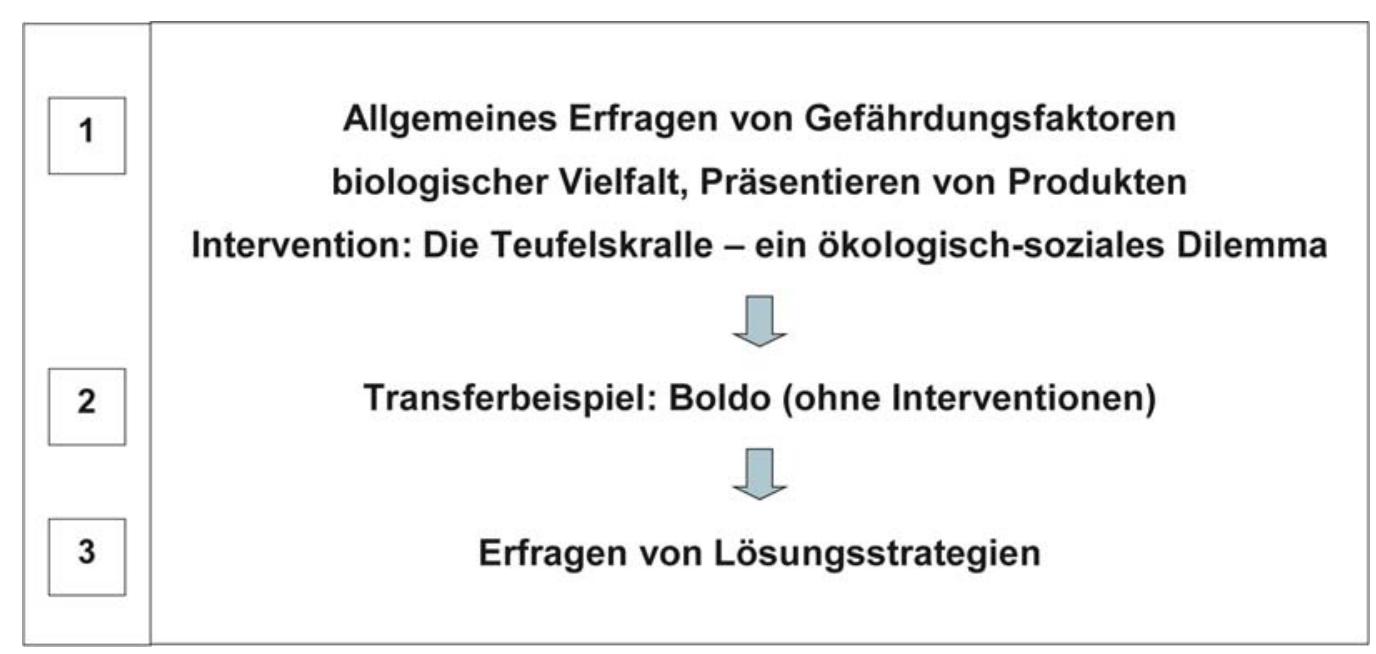

Abb. 6.1. Inhaltlicher Aufbau des Interview-Leitfadens.

\section{Datenaufbereitung und -auswertung}

Die Interviews wurden mittels eines Minidisc-Geräts aufgezeichnet und anschließend transkribiert. Die Transkription erfolgte Wort für Wort in normales Schriftdeutsch, wobei auch grammatikalische Fehler, Äußerungen des Zögerns und Nachdenkens (z.B. „ähm“), Umgangssprache und Elemente nonverbaler Kommunikation (z.B. Lachen) aufgenommen wurden. Die Originaltranskripte wurden mit Zeilennummern versehen, die in allen folgenden Auswertungsschritten mitgeführt wurden. In Anlehnung an Gropengießer (2001) wurden die Originaltranskripte in einem sich anschließenden Schritt „redigiert“, was der besseren Handhabbarkeit des ausführlichen Datenmaterials dient. Der Schritt des Redigierens umfasst eine grammatikalische Glättung der Texte sowie die Auflösung der Dialogform. Am Ende des Redigierprozesses stehen reine Schüleraussagen. Hinter jeder Aussage befinden sich in Klammern die Zeilennummern des Originaltranskripts, was einen direkten Vergleich mit dem genauen Transkript des Interviews in jedem Auswertungsschritt ermöglicht. Die Auswertung erfolgte computergestützt durch das Programm MAXqda (Kuckartz, 2004) in drei Analyseschritten, um den beiden unterschiedlichen Zielsetzungen gerecht zu werden:

1. Offenes Kodieren, induktiv (Glaser \& Strauss, 1998; Strauss, 1994), Identifikation von Schülervorstellungen auf unterschiedlichen Komplexitätsebenen; Auswahl von Prototypen für die identifizierten 
Subjektiven Theorien;

2. Systematische, deduktive Inhaltsanalyse (Miles \& Huberman, 1994), dazu Anlage von Kategorien, die der Struktur identifizierter Subjektiver Theorien entsprechen, unter anderem für ökologische, ökonomische und soziale Problemwahrnehmung;

3. Typologische Analyse nach Kluge (1999).

Es erfolgte zunächst eine Analyse nach Grounded Theory (Glaser \& Strauss, 1998) unter Ausführung des sogenannten „Offenen Kodierens“. Ziel dieses induktiven Analyseschritts ist die Identifizierung von Schülervorstellungen (auch in Form von Assoziationen, Kognitionen und Konzepten). Abbildung 6.2 veranschaulicht beispielhaft induktiv angelegte Kategorien. Zwei Prototypen, die aufgrund der induktiv identifizierten subjektiven Theorien ausgewählt wurden, dienen dabei der Veranschaulichung eines Typus „im Sinne eines konkreten Musterstücks“ (von Zerssen 1973, zitiert nach Kluge, 1999).

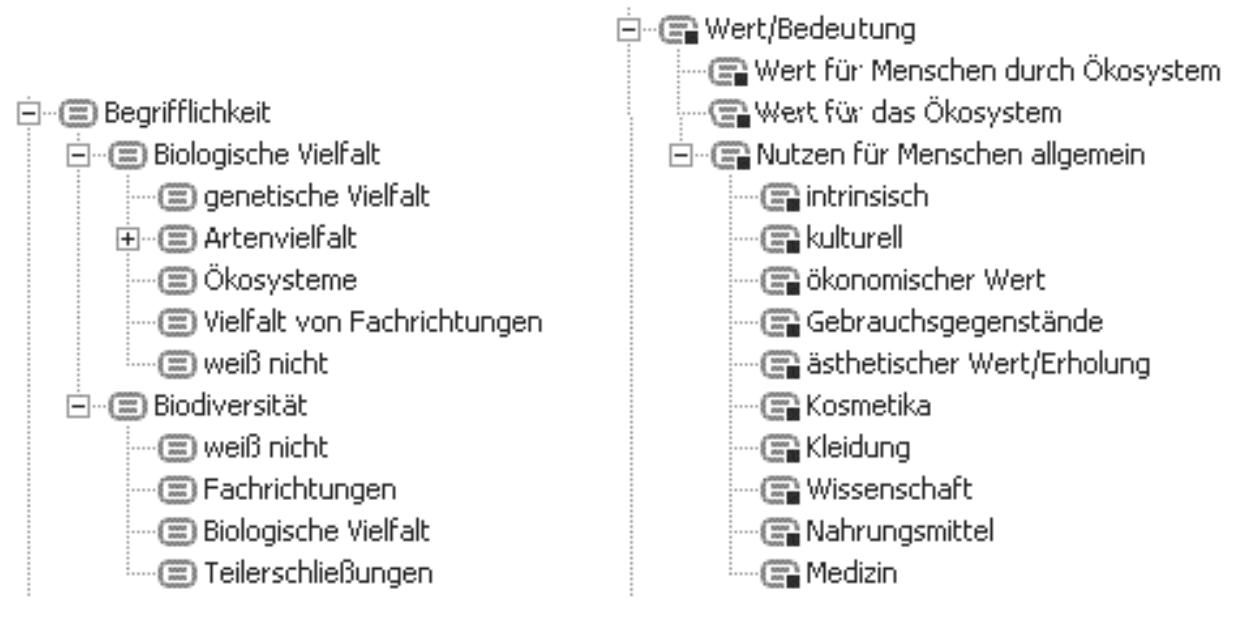

Abb. 6.2. Beispiele für induktiv angelegte Kategorien $\mathrm{zu}$ Schülervorstellungen zur Biodiversität und biologischen Vielfalt.

In einem zweiten Schritt werden deduktiv Kategorien erstellt, die Elemente ökologischsozialer Dilemmata sowie zentrale Elemente der identifizierten subjektiven Theorien repräsentieren (ökologische, ökonomische und soziale Problemwahrnehmung).

Eine typologische Analyse nach Kluge (1999) schließt die Analyse ab. Die deduktiv angelegten Kategorien ökologisch-sozialer Dilemmata und Subjektiver Theorien bilden die Grundlage einer Identifikation der Subjektiven Theorien bei weiteren Testpersonen und einer anschließenden Typologisierung.

Für jede induktiv wie deduktiv aufgestellte Kategorie wurden Definitionen erstellt, die von 
einer zweiten, unabhängigen Person überprüft und in ihrer Kongruenz bestätigt werden mussten. Durch diesen Schritt wird eine Intersubjektivität in der Auswertung gesichert. Gleiches gilt für die Definition von Kriterien, die der erstellten Typologie zu Grunde liegen (vgl. Tab. 6.3).
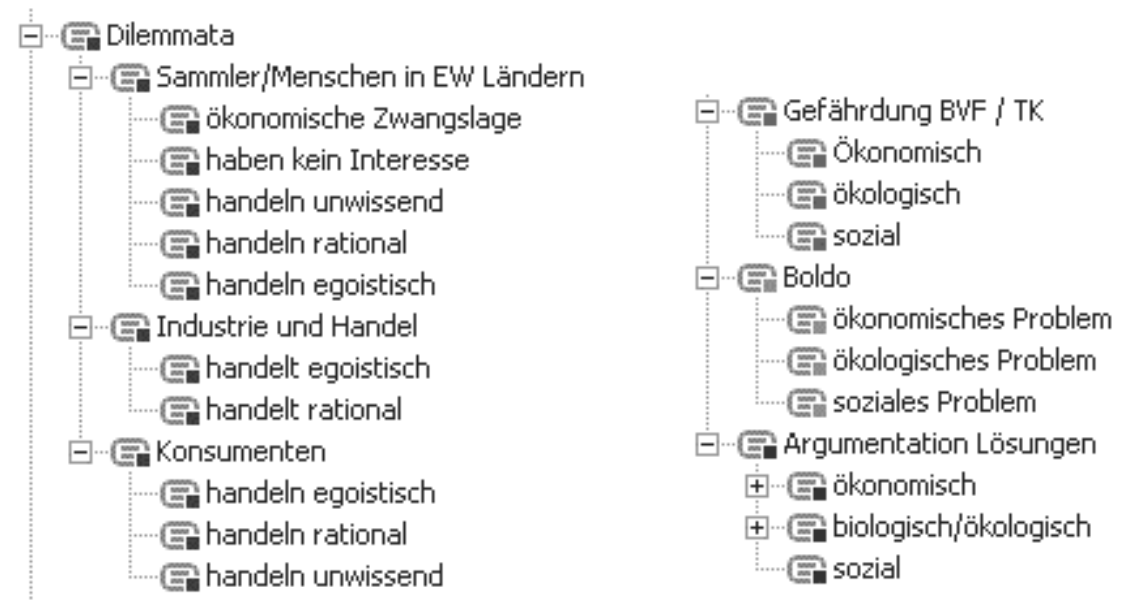

Abb. 6.3. Beispiele für deduktiv angelegte Kategorien zu ökologisch-sozialen Dilemmata.

\subsection{Ergebnisse}

Im folgenden Kapitel werden zunächst ausgewählte Ergebnisse zu Schülervorstellungen zum Begriff biologischer Vielfalt (bzw. Biodiversität) sowie zur Bedeutung und Gefährdung biologischer Vielfalt thematisiert. Als komplexeste Kategorie von Schülervorstellungen werden dann zwei Subjektive Theorien zum Biodiversitätsverlust vorgestellt. Anschließend wird eine Typologie auf Basis der identifizierten Subjektiven Theorien entwickelt und vorgestellt.

\section{Schülervorstellungen zu Begriff, Bedeutung und Gefährdung biologischer Vielfalt}

Keine(r) der Proband(inn)en gab an, den Begriff Biodiversität vor dem Interview gehört zu haben. Bei durch die Befragten geäußerten Vermutungen zeigt sich, dass eine prominente Assoziation zum Begriff „Biodiversität“ als „Vielfalt von biologischen Teildisziplinen“ besteht. Anna äußert folgende Assoziationen in Bezug auf Biodiversität: „[Biodiversität könnte sich darauf beziehen], dass man mit verschiedenen Mitteln in der Biologie vorgeht und dass eigentlich mit verschiedenen Beispielen [gearbeitet wird].“" (Anna, 85-86, 90) ${ }^{5}$. Lediglich Lilli assoziiert die Vielfalt des Lebens mit dem Begriff Biodiversität: „Vielleicht

\footnotetext{
${ }^{4}$ Alle angeführten Zitate sind als Redigierte Aussagen dargestellt.

${ }^{5}$ Die in Klammern angegebenen Zahlen bezeichnen die Zeilennummern im Originaltranskript.
} 
[ist Biodiversität] Lebensformen so [wie] Bakterien, Pilze, vielleicht die Unterschiede.“ (Lilli, 75-76).

Mit dem Begriff „biologische Vielfalt“ wird zumeist die Artenvielfalt von Tieren und Pflanzen sowie die ökosystemare Vielfalt assoziiert. Auch hier ist eine häufige Assoziation mit der „Vielfalt von biologischen Teildisziplinen“ festzustellen. Diese Interpretation erwies sich im Laufe des Interviews als zum Teil stabil und ging daher bei einigen Proband(inn)en über eine reine Assoziation hinaus zu Kognitionen bzw. einem einfachen Konzept. Johanna beispielsweise definiert biologische Vielfalt folgendermaßen: „Praktisch anwendendes Wissen, nicht so wie in Chemie oder Physik." (Johanna, 65-66). Bei der folgenden Beschreibung der Wichtigkeit von biologischer Vielfalt tritt dieses Konzept trotz der richtigen Assoziation mit Tier- und Pflanzenarten (im ersten Satz) wieder hervor, da sie dann (im zweiten Satz) offensichtlich biologische Fachdisziplinen als Möglichkeit sieht, die Tierund Pflanzenarten zu erhalten: „Erst einmal ist [biologische Vielfalt] wichtig, damit es verschiedene Tierarten gibt. Außerdem verschiedene Pflanzenarten. Um die [verschiedenen Pflanzen- und Tierarten] zu erhalten [ist biologische Vielfalt wichtig]. “(Johanna, 120-122). Die genetische Ebene biologischer Vielfalt wurde nur von einer Schülerin, Katja, direkt und einer weiteren Schülerin, Simone, indirekt genannt. Es handelt sich hier nicht um eine spontane Assoziation, sondern im Falle von Katja um die Begründung dafür, warum sie biologische Vielfalt als wichtig erachtet, bzw. im Falle von Simone, worin die Verschiedenheit von Arten begründet liegt. Bei der selten auftretenden Betrachtung der genetischen Ebene handelt es sich daher vermutlich eher um ein Konzept: „,...] wenn [...] eine Tierart irgendwie komplett gleich von Genen her [wäre] [...], dann würde sie wahrscheinlich auch schneller aussterben [...]. Weil, es muss dann ja nur eine Sache passieren, womit das Tier irgendwie nicht klarkommt. [...] Das würde die ganze Tierart betreffen.“ (Katja, 129-133). „[Facetten biologischer Vielfalt könnten] vielleicht Abwandlungen [sein]. [...] Wie bei Rosen zum Beispiel. Da gibt es ja die gezüchteten, dass man [die Rosen] einfach ein bisschen weiter züchtet und ein bisschen andere Arten [erhält].“(Simone, 163-166).

In Bezug auf den Wert und die Bedeutung biologischer Vielfalt waren prominente assoziierte Beispiele Nahrungsmittel, Medizin, Kosmetika und der ästhetische Wert biologischer Vielfalt.

Bei Gefährdung biologischer Vielfalt ist ein Konzept, das bei nahezu allen Befragten auftritt, mangelnder Platz als Ursache für Diversitätsverlust. Es wurde fast immer zu weit ausgeführt, als dass es sich um eine reine Assoziation handeln könnte und trat teilweise auch in späteren Phasen des Interviews wieder auf. Im „Platzkonzept“ wird die Bedrohung der biologischen 
Vielfalt vor allem in städtischen Räumen der Industriestaaten oder in der Ausbreitung von Monokulturen in Form von industrieller Landwirtschaft gesehen. So äußert Ayshe zunächst spontan auf die Frage nach möglichen Bedrohungen für die biologische Vielfalt: „In Städten [ist es besonders schwierig, die biologische Vielfalt zu erhalten]. Mir fällt da irgendwie sofort New York ein, die großen [Städte]. Ich weiß nicht, Hochhäuser und so und da wäre es ein bisschen schwierig. “ (Ayshe, 325, 329-330). Später, bei der Erläuterung der Rolle künftiger Generationen, greift sie auf dieses Konzept wieder zurück: „[...] Es werden ja immer mehr Häuser gebaut. Dass die [künftigen Generationen] auch darauf achten, dass [...] die Urwälder halt nicht kaputt gehen [könnte deren Bedeutung sein]. “ (Ayshe, 692-694). Interessant sind Konzepte über die Verantwortlichen für den Biodiversitätsverlust. Auffallend ist das Konzept der „egoistischen Sammler(innen)“ der Pflanzen, die als Modellorganismen dienen. Bei einer Reihe von Testpersonen traten negative pauschale Urteile über Menschen in ökologisch-sozialen Dilemmasituationen auf. So äußert beispielsweise Natascha: „[Das Verhalten der einzelnen Beteiligten im Falle Boldo würde ich als] selbstsüchtig [bewerten]. Vielleicht sollten [sie] auch einmal an andere Leute denken. Man sagt ja schon, wenn die Leute so weiter machen, haben wir bald keine Natur mehr, gar nichts. “ (Natascha, 955-958). Johanna bezeichnet das Verhalten der Beteiligten gar als „dumm“ (584). Auch Anna sieht die Verantwortung bei den Sammler(inne)n: „Die, die sie gepflückt haben [sind für den Rückgang der Pflanze verantwortlich zu machen]. “ (Anna, 357).

Zugleich nennen praktisch alle Proband(inn)en auch die wirtschaftliche Zwangslage der Sammler(innen). Auffallend ist dabei, dass manche Schüler(innen) lediglich auf Intervention hin die Zwangslage der Beteiligten erkennen. Es handelt sich hier vermutlich um interventionsgebundene Kognitionen, die häufig trotzdem negative Konnotationen in Bezug auf die Charakterisierung der Sammler(innen) aufweisen. Johanna erwägt die Zwangslage lediglich einmal auf Intervention hin mit folgenden Worten: „Die armen Leute versuchen ihr Geld zu kriegen und wenn sie wissen, dass sie für die Pflanze viel Geld kriegen, wenn sie die jetzt auf dem Schwarzmarkt verkaufen würden, dann würden die das auch machen. Sie denken sich, ja Gott, die ist ausgestorben, aber wenn ich jetzt [die Pflanze] schone, dann lebe ich vielleicht nächste Woche nicht mehr. “(Johanna, 673-686).

Dem gegenüber stehen komplexere Konzepte, in denen die Sammler(innen) als Personen in einer wirtschaftlichen Zwangslage gesehen werden. Diese Konzepte treten nicht nur interventionsgebunden auf, sondern werden selbstständig und mehrfach geäußert und teilweise in die Entwicklung von Lösungen eingebracht. Als Beispiel dienen die Gedanken von Simone: „Die Armut, die treibt die [Menschen] einfach soweit, selbst wenn sie es wüssten [dass die Pflanze bedroht ist], würde sie das nicht groß interessieren, weil die keine 
Wahl haben. Entweder verhungern oder irgendwie so an Geld kommen. " (Simone, 389-396). Später sagt sie erneut: „[Die Situation ist so ausgesprochen schwierig weil] einerseits [...] wissen die Leute schon, dass das nicht gut ist, wenn man alles auf einmal erntet, [...] aber andererseits ist die Nachfrage da und sie können damit Geld machen. Und dann ist es schwer, die Teufelskrallen da zu sehen und zu denken, die dürfen wir noch nicht ernten, weil die müssen erst einmal ein bisschen wachsen. [Das] ist halt schwer, also [das] ist dann schon ein innerer Konflikt." (Simone, 449-454). Dieser Gedanke wird mehrfach aufgegriffen: „Also die Sammler die machen das, um zu überleben. [...] Die machen das wirklich nur zum Überleben.“ (Simone, 583-584). Bei den Lösungen argumentiert sie entsprechend - das Konzept tritt wieder hervor: „Vielleicht [kann man] irgendwelche Alternativen anbieten, weil sie ja sonst auf der Straße stehen würden [und] arbeitslos sein und die haben ja oft Familie [...]. [Eine Lösung wäre] dass man [...] denen irgend welche Jobs zur Verfügung stellt. Damit sie auch wirklich den Anreiz haben, damit aufzuhören, weil sonst machen die das auch nur wegen dem Überleben und nicht weil sie Spaß daran haben [...]. “ (Simone, 666-674).

Neben den Sammler(inne)n von bedrohten Pflanzen sehen alle Testpersonen Industrie und Handel als verantwortliche Beteiligte in Bezug auf die Gefährdung der biologischen Vielfalt. Praktisch alle Befragten verfügen über das Konzept der „kapitalistischen Industrie“, das teilweise mehrfach genannt wird: „Aber die großen Konzerne nutzen das halt aus, dass die Armen für Geld ziemlich viel tun.“ (Sebastian, 503-507). „Die Hauptprobleme [in Bezug auf die Teufelskralle] sind, dass [...] sie trotz dieser hohen Bedrohung [stark] für die Wirtschaft genutzt wird, woran hauptsächlich die Menschen schuld sind, weil die einfach nur an Geld denken.“ (Johanna, 448-451). „Ich denke, größere Konzerne [sind für den Rückgang der Pflanze verantwortlich zu machen, die] nur auf den schnellen Gewinn aus sind und jetzt schnell Geld damit machen wollen. “ (Lilli, 370-371).

Interessanterweise tragen nur wenige Testpersonen das Konzept „kapitalistische Industrie“ weiter zur Verantwortlichkeit der Konsument(inn)en, die durch ihre Kaufentscheidungen zu einem erhöhten Druck auf die entsprechende Ressource beitragen können. Katja und Olaf erkennen Konsument(inn)en nur auf Intervention hin als Beteiligte, sodass an dieser Stelle lediglich von einer stark interventionsgebundenen Kognition ausgegangen werden kann.

Sebastian, Olaf, Andreas und Katja hingegen entwickeln diesen Gedanken selbstständig und ziehen ihn konsequent in weitere Überlegungen ein, sodass hier von einem Konzept gesprochen werden kann: „Die Leute hier in Europa oder [dort] wo diese Teufelskrallen Kapsel auch verkauft wird, setzen auf die Naturwirkstoffe und daher verkaufen die Pharmakonzerne das [Produkt].“ (Sebastian, 315-316) „,[Die Situation ist so ausgesprochen schwierig] weil eigentlich die Käufer davon überzeugt werden müssten, dass sie damit der 
Natur nicht helfen, sondern sie eher schädigen. “ (Sebastian, 324-328).

\section{Subjektive Theorien zum Biodiversitätsverlust}

Neben Assoziationen, Kognitionen und Konzepten konnten Subjektive Theorien zum Biodiversitätsverlust durch ökologisch-soziale Dilemmata identifiziert werden. Diese Theorien treten implizit oder explizit auf und beeinflussen die geäußerten Vorstellungen durch das gesamte Interview. Subjektive Theorien haben sich somit als über Konzepten, Kognitionen und Assoziationen stehende Vorstellungen erwiesen.

\section{Subjektive Theorie: Biodiversitätsverlust durch ökologische Ursachen}

Im Rahmen dieser Subjektiven Theorie werden sowohl Ursachen wie auch Folgen des Biodiversitätsverlusts überwiegend ökologisch betrachtet (Abb. 6.4). Zur Verdeutlichung sollen einige der Argumente von Johanna angeführt werden.

Johanna argumentiert durchgängig mit starker ökologischer Fokussierung. Bereits im Falle der Teufelskralle argumentiert sie trotz Interventionen vornehmlich ökologisch: „Ich denke, [dass manche dieser Produkte aus Wildsammlung stammen] bringt das Ökosystem durcheinander, weil die Natur nicht auf einen großen Abbau vorbereitet ist. [...] Dann stehen die Bäume nachher alle kahl und kommen nicht damit nach, [sich] wieder zu reproduzieren. Gedüngt werden die dann auch nicht, wenn sie wild wachsen. Das ist schon eine ziemlich große Bedrohung [...]. “(Johanna, 337-344). Zum Erhalt biologischer Vielfalt argumentiert sie wiederum stark ökologisch: „In Deutschland könnte [die Schwierigkeit der Erhaltung der biologischen Vielfalt] daran liegen, dass hier das Klima schlecht ist, weil hier zu wenig Sonne [und es] immer zu nass [ist], dass die Pflanzen daran kaputt gehen. [...] Und bei den wärmeren Ländern, haben die [Pflanzen] keine Zeit zu wachsen. “ (Johanna, 366371). Im Fortlauf des Interviews wird ihr gegenüber leitfadengemäß Boldo erwähnt und sie wird gebeten, das Problem in Bezug auf diese Pflanze zu erläutern: „Wenn die [Boldo Bäume] wild wachsen, [...] könnte [es] sein, dass mehrere Bäume oder Büsche ineinander wachsen, [so] dass die ziemlich schwer abzuernten sind." (Johanna, 566-570). Soziale Komponenten finden keine Erwähnung. Die Rolle der Sammler(innen) charakterisiert sie entsprechend ohne soziale Empathie: „[Das Verhalten der einzelnen Beteiligten ist in diesem Fall] dumm. Sie sollen [nur] das nehmen, was da ist und wenn sie den Baum stehen lassen würden, dann wäre die Kultivierung einfacher für die anderen Pflanzen. Und dann könnten sie zur Not, falls sie das schaffen, den Baum ernten. Aber wenn sie alles auf einmal wegmachen ist das nicht so klug.“ (Johanna, 584-588). Johannas Lösungen zielen auf Gesetze und Kontrollen ab. Sie nennt keine Lösungsvorschläge, die auf die wirtschaftliche 
und soziale Situation der Sammler(innen) vor Ort abzielen. Nur an einer Stelle im Interview zieht sie in Erwägung, dass die Situation in Bezug auf die Dilemmabeispiele von lokaler Armut abhängen könnte. Diesen Gedanken greift sie bei der Erarbeitung von Lösungen jedoch nicht wieder auf, er ist offensichtlich nicht Teil ihrer Subjektiven Theorie zum Biodiversitätsverlust.

\section{Subjektive Theorie: Biodiversitätsverlust durch soziale Ursachen}

Unter den Proband(inn)en konnte eine zweite Subjektive Theorie identifiziert werden, die soziale Aspekte als Ursache für den Verlust der Biodiversität mit einbezieht. Als Beispiel soll das Profil von Katja (Abb. 6.4) dienen.

Bei Katja stehen soziale Argumente im Vordergrund, die sie mit ökonomischen oder ökologischen Argumenten verknüpft. In Bezug auf die Teufelskralle argumentiert sie zunächst überwiegend ökologisch, erkennt aber auch eindeutig die ökonomische Zwangslage als Hauptelement des Dilemmas: „Ich glaube [die Situation ist so schwierig, weil] die Menschen, die das pflücken, [...] irgendwo dieses Geld brauchen, weil sie sonst keinen Lebensunterhalt hätten. Und dass sie irgendwie nicht die Chance haben, irgend etwas anderes zu machen, dass sie das [Geld] wirklich brauchen [macht die Situation so ausgesprochen schwierig]. “ (Katja, 398-401). In Bezug auf Boldo sieht sie die soziale Komponente verknüpft mit ökonomischen Mechanismen: "Ich schätze, dass es [in Bezug auf die Hauptbeteiligten] wieder ungefähr genauso abläuft, dass es irgend welche Sammler gibt, die Lohn dafür kriegen und dass es dann zu uns exportiert wird. “ (Katja, 463-465). Die Rolle der Sammler(innen) charakterisiert sie wie folgt: „Ich denke, dass die Menschen, [...] vielleicht eigentlich einfach nur um ihren Lebensunterhalt kämpfen und sich im Prinzip nicht weiter Gedanken darüber machen, was sie da wirklich tun. “ (Katja, 315-317). Lösungen generiert Katja dann wiederum aus einer Kombination von ökologischen und sozialen Aspekten, indem sie die Bewusstseinsbildung der Beteiligten auf beide Aspekte bezieht: „[Man könnte die Verbraucher] bei den Pflanzen [darüber aufklären], dass der Bestand zurück geht [und dass] bei Tieren der Bestand zurückgeht. Und ihnen klar machen, dass es so nicht geht [...]. [Man könnte] den Menschen dann irgendwelche Alternativen bieten, dass sie jetzt nicht unbedingt echte Tierfelle haben müssen, sondern irgend etwas anderes." (Katja, 567-570).

\section{Erstellen einer Typologie}

Um ökologisch-soziale Dilemmata zu lösen, ist es entscheidend, ökologische, ökonomische und soziale Phänomene in inhaltliche Beziehung zu setzen (Retinitätsforderung, WCED, 
1987) ${ }^{6}$. Bei der Identifikation der beiden prominenten Subjektiven Theorien ließ sich vermuten, dass eine Gruppe der Befragten dazu neigt, auf ökologische Aspekte zu fokussieren, während andere offen für soziale Argumente sind. Um dieser Vermutung systematisch nachzugehen, wurde eine Analyse der Argumentationen in den drei unterschiedlichen Phasen des Interviews vorgenommen. Ökonomische, ökologische und soziale Argumentationsweisen wurden gemäß des Leitfadens in drei Blöcken systematisch erfasst (vgl. Abb. 6.1):

1. Auf allgemeinem Niveau (Vorstellungen zu Begriff, Bedeutung und Gefährdung biologischer Vielfalt) und dem Beispiel Teufelskralle,

2. auf der Stufe des Transferbeispiels Boldo und

3. bei der Generierung eigener Beispiele und Lösungsstrategien.

Es wurden angelehnt an die beiden Subjektiven Theorien zwei Typenklassen identifiziert: 1 . ein ökologisch orientierter Typ, 2. ein Retinitäts-Typ. Von letzterem Typus wurde dann als Typ 3 ein ökologisch-sozialer Typus unterschieden. Proband(inn)en dieser dritten Typenklasse schenkten ökonomischen Aspekten weniger Beachtung und konnten so nicht als „echte“ Retinitäts-Typen klassifiziert werden. Eine Person ließ sich keinem Typus zuordnen und wurde unter die Kategorie „indifferent“ gefasst. Das Kästchenraster (Abb. 6.4) ist dabei lediglich eine vereinfachende Zuordnungshilfe. Die Zuordnung einer Person erfolgte auf inhaltliche, qualitative Kriterien hin. Es handelt sich bei der Typisierung also um eine qualitative Zuordnungsmethode, die sich nicht ausschließlich an der (quantitativen) Anzahl der Nennungen orientiert.

Prototypen veranschaulichen die Charakteristika eines Typus in besonders deutlicher Weise. Die Kriterien, die für den jeweiligen Typ (ökologischer Typ, Retinitäts-Typ oder ökologisch-sozialer Typ) festgelegt wurden, sind bei den ausgewählten Prototypen ohne Abweichungen zu finden. Die Prototypen sind dabei Realtypen, da sie sich an den tatsächlichen Äußerungen orientieren und nicht überzeichnet wurden, um die typologischen Besonderheiten darzustellen. Die Kriterien, die für jeden Typ erstellt wurden, sind in Tabelle 6.3 aufgeführt.

${ }^{6}$ Das Prinzip der Retinität bezeichnet die Gesamtvernetzung der drei Bereiche Nachhaltiger Entwicklung: Ökologie, Ökonomie und Soziales (Rat von Sachverständigen für Umweltfragen (SRU), 1994). 


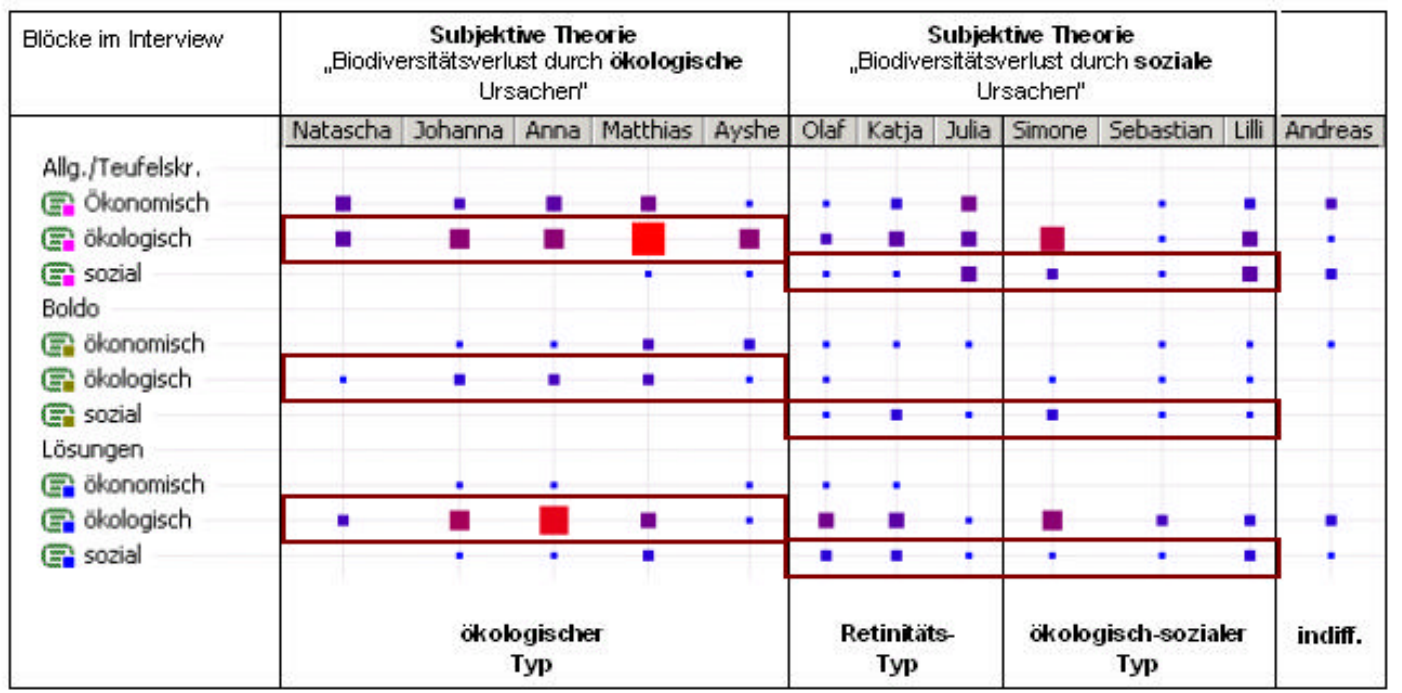

Abb. 6.4. Typen und deren Argumentationsweisen in den drei Phasen des Interviews. Je größer die Markierung, desto zahlreicher sind die Nennungen innerhalb einer Kategorie.

Tab. 6.3. Kriterien für die Bildung der Typen.

\begin{tabular}{|c|c|c|}
\hline Typus & Kriterien & $\begin{array}{l}\text { Zugehörige } \\
\text { (Prototypen } \\
\text { fett) }\end{array}$ \\
\hline $\begin{array}{l}\text { Ökologischer } \\
\text { Typus }\end{array}$ & $\begin{array}{l}\text { - Ökologische Argumentationen: Auf allen drei Stufen } \\
\text { vorhanden } \\
\text { - Ökonomische oder soziale Argumentationen: Auf keiner } \\
\text { Stufe stärker als ökologische Argumentationen } \\
\text { - Gleiche Intensität ökologischer Argumentationen mit } \\
\text { ökonomischen oder sozialen Argumentationen auf einer } \\
\text { Stufe: Ausgleich durch Dominanz ökologischer Argumente } \\
\text { auf den beiden anderen Stufen }\end{array}$ & $\begin{array}{l}\text { Johanna } \\
\text { Natascha } \\
\text { Anna } \\
\text { Matthias } \\
\text { Ayshe }\end{array}$ \\
\hline $\begin{array}{l}\text { Retinitäts- } \\
\text { Typus }\end{array}$ & $\begin{array}{l}\text { - Soziale Argumentationen: Auf allen drei Stufen vorhanden } \\
\text { - Soziale Argumentationen wechselnd in Verbindung mit } \\
\text { ökologischen oder ökonomischen Argumentationen } \\
\text { - Ökologische und ökonomische Argumentationen sind nicht } \\
\text { zwingend durchgängig vorhanden }\end{array}$ & $\begin{array}{l}\text { Katja } \\
\text { Julia } \\
\text { Olaf }\end{array}$ \\
\hline $\begin{array}{l}\text { Ökologisch- } \\
\text { sozialer } \\
\text { Typus }\end{array}$ & $\begin{array}{l}\text { - Soziale Argumentationen: Auf allen drei Stufen vorhanden } \\
\text { - Ökologische Argumentationen: Auf allen drei Stufen } \\
\text { vorhanden } \\
\text { - Verhältnis ökologische und soziale Argumentationen: Keine } \\
\text { durchgängige, eindeutige Gewichtung }\end{array}$ & $\begin{array}{l}\text { Lilli } \\
\text { Simone } \\
\text { Sebastian }\end{array}$ \\
\hline Indifferent & - Keine eindeutige Zuordnung möglich & Andreas \\
\hline
\end{tabular}

\section{Der ökologische Typus}

Zentrales Kriterium für die Zuordnung zu diesem Typus ist ein Aufgreifen ökologischer Argumentationen in allen drei für die Auswertung relevanten Blöcken (s. Abb. 6.4 und Tab. 6.3). Es werden somit sowohl für die Beispiele Teufelskralle und Boldo wie auch für die entwickelten Lösungsstrategien überwiegend ökologische Argumente herangezogen. Bei 
einigen Testpersonen treten in maximal einer Phase des Interviews ökologische Argumentationen gleich stark auf wie beispielsweise ökonomische Argumentationen (vgl. Abb. 6.4, Natascha, Stufe 1 oder Matthias, Stufe 2). Ausschlusskriterium für eine Zugehörigkeit zur Gruppe des ökologisch orientierten Typus ist das Fehlen ökologischer Argumentationen in einem oder mehreren der drei Interview-Blöcke. Dem ökologisch orientierten Typus können fünf Schüler(innen) zugeordnet werden (s. Abb. 6.4): Natascha, Johanna, Anna, Matthias und Ayshe. Als Prototyp für den ökologischen Typus ist Johanna anzusehen, deren ökologisch orientierte Argumente gemäß der Subjektiven Theorie „Biodiversitätsverlust durch ökologische Ursachen“ in allen Phasen des Interviews überwiegen. Trotz der Nennung von ökonomischen und sozialen Argumenten (die jedoch überwiegend auf der Ebene der Assoziationen oder teils interventionsgebundenen Kognitionen verbleiben) ist somit eine regelmäßige Dominanz ökologischer Komponenten festzustellen.

\section{Der Retinitäts-Typus}

Bei diesem Typus sind soziale Argumentationen in allen drei ausgewerteten Phasen des Interviews vorhanden. Ökologische und ökonomische Argumentationen werden von den diesem Typus zugeordneten Testpersonen jedoch nicht durchgängig herangezogen. Diesem Typus können drei Personen zugeordnet werden: Katja, Olaf und Julia. Katja kann als Prototyp für den Retinitäts-Typus angesehen werden, anhand ihres Profils wurde die Subjektive Theorie „Biodiversitätsverlust durch soziale Ursachen“ dargestellt. Soziale Argumentationen sind bei Katja durchgängiges Prinzip, wobei soziale Zusammenhänge in der Regel nicht isoliert, sondern in Kombination mit ökologischen und /oder ökonomischen Faktoren gesehen werden.

\section{Der ökologisch-soziale Typus}

Personen dieses Typus verfügen ebenfalls über die Subjektive Theorie „Biodiversitätsverlust durch soziale Faktoren“. Allerdings spielen ökonomische Faktoren eine weniger gewichtige Rolle als bei dem Retinitäs-Typus, der alle drei Facetten Nachhaltiger Entwicklung in den Blick nimmt. Das Hauptkriterium für diesen Typus sind folglich soziale und ökologische Argumentationen in allen drei Phasen des Interviews. Es wird, auf das gesamte Interview bezogen, weder ökologischen noch sozialen Argumenten Vorzug gegeben. Entweder tritt eine durchweg gleichwertige Nennung ökologischer und sozialer Argumentationen auf (s. Abb. 6.4, Lilli) oder die Personen schwanken zwischen dem Schwerpunkt ökologisch und sozial (s. Abb. 6.4, Simone). Simone argumentiert zwar ausschließlich ökologisch-sozial 
(ohne ökonomische Argumente anzuführen), doch dominieren auf den Stufen 1 und 3 ökologische, auf Stufe 2 soziale Argumentationen. Insgesamt konnten diesem Typus drei Personen zugeordnet werden: Simone, Sebastian und Lilli. Als Prototyp des ökologischsozialen Typus ist Lilli anzusehen. Lilli führt auf allen drei Stufen ökologische und soziale Argumente gleichermaßen auf und setzt ihren Argumentationsschwerpunkt nicht auf eine der beiden Facetten.

\section{Indifferent}

Bei einer Person, Andreas, kann keine eindeutige Zuordnung zu einem der vorgestellten Typen vorgenommen werden. Er wird unter die Kategorie „indifferent“ gefasst. Andreas erwähnt beim Interventionsbeispiel Teufelskralle ökologische, ökonomische und soziale Komponenten (unter geringfügig schwächerer Nennung ökologischer Aspekte). Bei Boldo greift er nur ökonomische, bei Lösungsstrategien nur ökologische und soziale Aspekte an hier mit einer leichten Gewichtung auf ökologischen Aspekten.

\section{Konzepte und Typen}

Nach Groeben et al. (1988) nehmen Subjektive Theorien eine Realitätskonstituierung vor. Sie beeinflussen somit neu erworbene Konzepte, Kognitionen und Assoziationen und stehen mit ihnen in Zusammenhang.

Die einzigen beiden Probandinnen, die biologische Vielfalt auf allen drei Ebenen Artenvielfalt, ökosystemare Vielfalt und genetische Vielfalt erkennen, gehören zum Retinitäts- bzw. ökologisch-sozialen Typus. Keiner Testperson des ökologischen Typus gelingt diese vollständige Erfassung. Während das „Platzkonzept“ bei allen Proband(inn)en außer Andreas auftaucht, scheinen Konzepte, die sich auf Verantwortliche für den Biodiversitätsverlust beziehen, wieder eine typologische Abhängigkeit aufzuweisen. Das Konzept der egoistischen Sammler(innen) tritt, abgesehen von Matthias, bei allen Zugehörigen des ökologischen Typus auf. In den beiden anderen Typenklassen, die sich durch eine höhere soziale Affinität auszeichnen, nennt lediglich Julia - und das nur ein einziges Mal - egoistische Motive der Sammler(innen).

Die wirtschaftliche Zwangslage der Sammler(innen) wird zwar von allen Testpersonen genannt, häufig jedoch nur auf Intervention (vgl. Kap. 4.1). Auf der Ebene der Konzepte beziehen Simone, Sebastian, Lilli, Olaf, Katja, Julia und Andreas diese Überlegung mit in ihre Ausführungen ein. Alle diese Testpersonen sind entweder dem ökologisch-sozialen oder dem Retinitäts-Typus zugeordnet. Alle Proband(inn)en der beiden genannten Typen verfügen über das Konzept der ökonomischen Zwangslage. 
Das Konzept der „kapitalistischen Industrie“ wird ebenfalls von allen Schüler(inne)n genannt. Jedoch lediglich Sebastian, Katja, Olaf und Andreas tragen es weiter hin zur Verantwortlichkeit der Verbraucher(innen) im Prozess des Handels und Vertriebs gefährdeter Medizinalpflanzen. Die vier genannten Personen gehören alle (bis auf Andreas als nicht klassifizierte Person) entweder dem ökologisch-sozialen oder dem Retinitäts-Typus an.

\subsection{Diskussion - Schülervorstellungen zur Biodiversität als Herausforderung für eine Bildung für eine Nachhaltige Entwicklung}

Die aufgezeigten Vorstellungen von Schüler(inne)n zur Biodiversität und deren Gefährdungs- und Erhaltungsmöglichkeiten sind bedeutsam für eine empirisch fundierte Bildung zur Biodiversität. Zunächst werden die identifizierten Vorstellungen zu Begriff, Bedeutung und Gefährdung von biologischer Vielfalt innerhalb der Stichprobe im Hinblick auf ihre Relevanz für eine Biodiversitäts-Bildung diskutiert. Es folgt eine Diskussion der Typologie und deren Bedeutung für Bildungsmaßnahmen zur Biodiversität.

Assoziationen, Kognitionen und Konzepte zu Begriff, Bedeutung und Gefährdung biologischer Vielfalt

Ergebnisse dieser qualitativen Studie haben keine verallgemeinerbare Aussagekraft. Dennoch können sie dazu dienen, Vermutungen über prominente Schülervorstellungen zu Biodiversität in der elften Klasse zu formulieren. Im Folgenden sollen die identifizierten Assoziationen, Kognitionen, Konzepte, Subjektiven Theorien und Typen hinsichtlich ihrer Relevanz für einen naturwissenschaftlichen Unterricht diskutiert werden.

Es lässt sich vermuten, dass der Begriff „Biodiversität“ nicht bekannt ist. Eine grundlegende Begriffsklärung wäre daher im Rahmen einer Bildung für Nachhaltige Entwicklung wünschenswert. Auf eine möglicherweise weit verbreitete Fehlinterpretation des Begriffs als „Vielfalt von Fachrichtungen“, die auch den Begriff „biologische Vielfalt“ betreffen kann, sollte Bezug genommen werden. Die Interpretation des Begriffs „biologische Vielfalt“ scheint ansonsten keine Schwierigkeiten zu bereiten, wobei eine weitgehende Reduktion auf die Ebenen der Arten- und Ökosystemvielfalt festzustellen ist. Eine Erarbeitung besonders der genetischen Ebene und ein systematisches „In-Beziehung-Setzen“ der drei Ebenen biologischer Vielfalt ist für eine Bildung für Nachhaltige Entwicklung zentral, um „Biodiversität“ (bzw. biologische Vielfalt) zu verstehen. Die Anknüpfung an lebensweltliche Kontexte der Schüler(innen) bietet sich an: Verbindungen zwischen der biologischen Vielfalt 
und der Lebenswelt der Schüler(innen) wie Nahrungsmittel, Medizin und Kosmetika sind mögliche Zugänge.

Das weit verbreitete „Platzkonzept“ weist auf eine vereinfachte Sicht auf Gründe für den Verlust der Biodiversität hin. Zwar sind die Gewinnung von Weideland und die Anlage von Monokulturen Gründe für einen Rückgang der Biodiversität, doch stehen dahinter ökonomische und soziale Fragestellungen, die durch eine alleinige Vermeidung von raumeinnehmenden (Agrar-) Industrien nicht gelöst werden können. Vor allem das Konzept des prioritären Biodiversitätsverlusts im städtischen Raum greift zu kurz.

Das Konzept der „egoistischen Sammler(innen)“ ist als problematisch anzusehen. Es zeugt von einer mangelnden Fähigkeit zur Perspektivübernahme - eine Voraussetzung zu einem Verständnis intragenerationaler Gerechtigkeit im Sinne Nachhaltiger Entwicklung. Ein Fokus bei einer Bildung zur Nachhaltigen Entwicklung zur Biodiversität sollte daher auf der Darstellung der ökonomischen Zwangslage von Menschen in Entwicklungsländern liegen. Die Ergebnisse zeigen, dass allen Proband(inn)en dieser Gedanke zumindest auf Intervention hin zugänglich ist - wenn auch nur wenige Testpersonen diese Vorstellung auf der Ebene eines Konzepts äußern.

Das Konzept „kapitalistische Industrie“ lässt auf eine starke externe Verantwortungszuschreibung schließen. Auch wenn Wirtschaft und Handel beim Biodiversitätsverlust zweifelsohne eine zentrale Bedeutung zukommt, kann dieses Konzept hinderlich sein, alternative wirtschaftliche Ansätze im Sinne Nachhaltiger Entwicklung zu berücksichtigen. Die Förderung einer positiven Perspektive auf eine Nachhaltige Wirtschaft wäre im Rahmen von Biodiversitätsbildung wünschenswert.

Nur wenige Schüler(innen) sehen die eigene Verantwortung als Konsument(in). An dieser Stelle kann in einer Bildung zur Biodiversität jedoch eine realistische Handlungsperspektive aufgezeigt werden, die starke lebensweltliche Bezüge für die Lerner(innen) aufweist.

Subjektive Theorien „Biodiversitätsverlust durch ökologische Ursachen“ und „Biodiversitätsverlust durch soziale Ursachen“

Die Subjektive Theorie „Biodiversitätsverlust durch ökologische Ursachen“ zeichnet sich dadurch aus, ökologischen Argumenten durch das gesamte Interview den Vorzug zu geben. Ökonomische und vor allem soziale Argumente werden nicht gleichermaßen genannt. Die starke ökologische Fokussierung kann, wie beim Prototypen Johanna, zu einem Unverständnis gegenüber sozialen Zwangslagen benachteiligter Gruppen führen. Anhand von Johannas Profil lässt sich bei der genaueren Analyse der Aussagen besonders deutlich nachweisen, dass eine ständige Bezugnahme auf ökologische Sachverhalte nicht ausreicht, 
um Gründe für den Verlust der biologischen Vielfalt adäquat zu rekonstruieren.

Die spezifische Argumentation des ökologisch orientierten Typs legt die Notwendigkeit einer Integration ökonomischer und sozialer Komponenten in den naturwissenschaftlichen Unterricht nahe. Gayford (2000) beschreibt jedoch die Unsicherheit von Biologielehrer(inne)n in England gegenüber sozialen und ökonomischen Komponenten des Themas. Ähnliche Auffassungen sind bei deutschen Lehrer(inne)n zu vermuten. Vor allem für Personen, die dem ökologisch orientierten Typus zuzuordnen sind, ist es wichtig, die Legitimität sozialer und ökonomischer Argumente für reale Gestaltungsaufgaben in Fragen der Biodiversität wahrzunehmen und zu verstehen. Gleichzeitig dürfen aber auch ökologische Zusammenhänge nicht aus dem Blick verloren werden: Gerade beim Prototyp Johanna zeigen sich naive und teilweise falsche Vorstellungen zu ökologischen Zusammenhängen. Die Erweiterung und ggf. Modifizierung ökologischer Kenntnisse ist gleichermaßen Aufgabe einer Bildung zur Biodiversität.

Die Subjektive Theorie „Biodiversitätsverlust durch soziale Ursachen“ zeichnet sich dadurch aus, dass soziale Komponenten ökologisch-sozialer Dilemmata systematisch in die Argumentationen einbezogen werden. Es ist positiv hervorzuheben, dass es diese Subjektive Theorie offensichtlich eher erlaubt, die Perspektive von Menschen in ökonomischen Zwangslagen einzunehmen. Die Subjektive Theorie „Biodiversitätsverlust durch soziale Ursachen“ führt so zu einem besseren Verständnis des Verlusts der Biodiversität. Es wird deutlich, dass die Erweiterung einer rein ökologisch geprägten Theorie hin zu einer Theorie sozialer und ökonomischer Perspektiven sinnvoll ist. Auch dies stützt die Notwendigkeit eines Biologieunterrichts, der über rein ökologische Betrachtungen des Biodiversitätsverlusts hinaus geht: Eine ökologische Fokussierung könnte bei Schüler(inne)n den Eindruck erwecken, dass soziale Argumente keine gleichwertige Legitimität besitzen. Bei der Thematisierung von Biodiversität sollte das Potenzial von Subjektiven Theorien wie „Biodiversität durch soziale Ursachen“ genutzt werden.

\section{Typologische Analysen}

Für die Konzipierung von Bildungsmaßnahmen zur Biodiversität kann es hilfreich sein, sich der Möglichkeit unterschiedlicher Typen unter den Lernenden bewusst zu sein. Eine Typenbildung kann unter anderem von großem Nutzen sein, um eine adressatenorientierte Umweltbildung zu erleichtern (Bögeholz, 2001; Bögeholz \& Barkmann 1999).

Die entwickelte Typologie baut auf identifizierten Subjektiven Theorien auf und wird durch die identifizierten Konzepte gestützt. Es ist konsistent, dass gerade die Konzepte, die soziale Empathie voraussetzen, auch nur in den beiden Typen auftreten, die soziale Elemente 
zulassen. Die Typologie weist darauf hin, dass die beiden Subjektiven Theorien keine rein individuellen Muster zu sein scheinen, sondern unter Schüler(inne)n der elften Klasse prominent sind. Bei einer Bildung für Nachhaltige Entwicklung, insbesondere zur Biodiversität, sollten sich Lehrende der Situation bewusst sein, dass soziale und ökonomische Probleme zumindest von einem Teil der Lernenden nur gegen ihre Subjektiven Theorien einbezogen werden können. Deutlich wird hier, wie entscheidend die Beachtung sozialer Aspekte beim Verständnis der Ursachen für den Biodiversitätsverlust ist: Das Konzept der „egoistischen Sammler(innen)“ tritt beispielsweise ausschließlich bei Testpersonen auf, die dem ökologischen Typus angehören. Gleichzeitig zeigen derartige Konzepte, wie wichtig eine Einbeziehung sozialer und ökonomischer Faktoren ist, um im Sinne einer Nachhaltigen Entwicklung reale Lösungs-, und Handlungsmöglichkeiten aufzuzeigen um intra- und intergenerationale Gerechtigkeit zu ermöglichen. 

How do pupils in Chile and Germany perceive resource dilemmas? ${ }^{1}$

\subsection{Abstract}

Biodiversity loss is a global phenomenon and biodiversity education, therefore, a global challenge. In some regions of the world, biodiversity is particularly threatened. Especially in such biodiversity hotspots, among them Chile, resource dilemmas are serious threats to biodiversity. In such dilemmas, natural resources are depleted for economic interest which is, in many cases, required income. Therefore, in order to fully understand biodiversity loss, economic and social aspects must be considered. Albeit so, little is known thus far about learning prerequisites for biodiversity, especially under an interdisciplinary perspective. This paper presents a qualitative, in-depth interview study that investigated sixteen to eighteenyear-old Chilean and German learners' perception of biodiversity and its loss $(n=24)$. Firstly, pupils' cognitive frameworks were analysed and secondly, where possible, assigned to different levels of complexity. Moreover, an "ecological type", an "economic type" and a "balanced type" emerged from the data, showing the difference in the perception of biodiversity and its loss. The results showed that most pupils focussed either on ecological or on economic aspects of biodiversity loss. Pupils who concentrated on ecological aspects applied weak ecological knowledge more often. Nevertheless, some pupils succeeded in integrating the ecological, economic, and social aspects. Chilean pupils seemed to have greater difficulties to recognize social aspects of biodiversity loss. As an implication for future research, reasons for the different perceptions of biodiversity loss could be investigated, for instance, in a quantitative study and with a larger sample of Chilean and German adolescents.

\subsection{Introduction}

Biodiversity conservation is a central challenge for sustainable development (UNCED, 1992; UNEP, 1995; Wilson, 2001). Therefore, numerous national and international legal frameworks aim at conserving biodiversity. When biodiversity is threatened, in most cases

\footnotetext{
${ }^{1}$ Source: Menzel, S., \& Bögeholz, S. (2007). The Loss of Biodiversity as a Challenge for Sustainable Development: How do pupils in Chile and Germany perceive resource dilemmas? Manuscript accepted for publication, major revisions required (Research in Science Education).
} 
ecological, economical and social aspects are closely interwoven. Therefore, all three pillars of sustainable development (ecology, economy, society) must be considered when adequate biodiversity conservation is to be achieved.

One of the most important international legislations to prevent biodiversity loss is the Convention on Biological Diversity (CBD, 1992). The CBD aims at conserving biodiversity through a likewise consideration of social, economic, and ecological aspects of biodiversity loss. Moreover, article 13a of the CBD explicitly takes into account the central role that education plays in biodiversity conservation. In order to comply with this objective, schools are pivotal institutions for communicating the importance of biodiversity education. Due to the complexity of the issue, this is by no means an easy task.

For learners, several aspects of the biodiversity concept might be challenging. First, the fact that the terms "biodiversity" and "biological diversity" are identical in meaning might confuse learners. Furthermore, both terms are commonly understood to be a synonym for "the variety of species". However, the literature suggests that such an understanding has shortfalls because a modern ecological definition incorporates three different levels of biodiversity of equal importance namely, diversity of species, diversity of ecosystems, and genetic diversity (e.g. Townsend et al., 2003). The loss of biodiversity occurs within all three levels of biodiversity (UNCED, 1992).

A second challenge for learner might be the fact that reasons for biodiversity loss are complex. Although the term „biodiversity“ suggests an ecological focus, reasons for biodiversity loss surround complex ethical, economic and social issues (e.g. UNEP, 1995; WCED, 1987; Wilson, 2001). Therefore, to appropriately understand drivers for and consequences of biodiversity loss, learners have to deal with interdisciplinary and complex issues.

The interaction of ecological, economic, and social aspects becomes especially apparent in some regions that are more affected by biodiversity loss than others. Such regions commonly show a combination of extremely diverse species, genes and ecosystems on the one hand and poverty coupled to a rapid population growth on the other. The interaction of these factors often results in a high pressure being placed on biodiversity. As a consequence, thirty-four of these areas have been defined as "biodiversity hotspots" (Mittermeier et al., 2004; Myers et al., 2000). Central Chile is an example of a region that contains such a hotspot. Here, the pressure on biodiversity is, among other things, due to Chile’s emerging market, one, which is based mainly on an export of raw materials such as copper or pinewood (Scholz, 1999). Unfortunately, the exploitation of raw materials and natural resources often happens under poor ecological standards, which threatens local biodiversity. 
Furthermore, threats to the environment in Chile are also rooted in social problems often associated with emerging markets. For example, felling of native forests or exploitation of wild herbs are classical "resource dilemmas" that lead to a depletion of natural resources due to social pressures (Ernst, 1997; Hardin, 1968). In such resource dilemmas, a common, openaccess resource is used, and in many cases overused, by multiple parties. Resource dilemmas are difficult to solve as beneficiaries often depend on the respective natural resource in economic terms. In this regard, "biodiversity loss" is a topic that shows close relationship with Chilean pupils' natural environment, much more so than in the case of pupils living in industrialized countries, such as Germany.

Resource dilemmas are not limited to Chile. They are a global phenomenon and generally among the main drivers for biodiversity loss (e.g. Cincotta et al., 2000; WCED, 1987). In most cases, consequences of the overuse of a resource are unknown to the individual exploiting the resource, as damages tend to occur delayed (Ernst, 1997; Hardin, 1968). Today, globalized markets aggravate the overuse of natural resources. Due to global market patterns, plants and animals that were formerly used only on a local scale are now available globally and in large quantities (Shiva, 2000). Therefore, the pressure on common goods that are usually not cultivated is a serious threat to biodiversity.

In the current study, two examples of resource dilemmas were applied to exemplify biodiversity loss: the South African Devil's Claw (Harpagophytum procumbens) and the South American Boldo (Peumus boldus). Both species are medical plants, which are traded on a large scale and collected from the wild.

\subsection{Theoretical background}

\section{Biodiversity Education}

Since biodiversity issues are so prevalent as well as complex, biodiversity has been identified as a major challenge for science education for more than ten years now (Mallow, 1994; Mayer, 1996). For instance, in terms of contents of biodiversity education, Kassas (2002) has suggested that all three levels of biodiversity should be included and that the subject should also take economic, social and ecological facets into account. However, in science education research, ecological aspects of biodiversity, especially the variety of species, are emphasized. In one account, Balmford et al. (2002) described how North American pupils' knowledge of local bird species is clearly weaker than that of Pokémon“species”. Numerous studies support Balmford's findings. For example, Jäkel and Schaer (2004) have identified German middle school pupils' low knowledge of local plant and 
animal species, while similar findings for British A level pupils have been reported by Bebbington (2005). In a Spanish context, Salinas-Hernández (2002) has explored pupils' prior knowledge and textbook contents of ecological aspects pertaining to biodiversity. Apart from these findings, Lindemann-Matthies (2002b, 2006) included psychological aspects in a study and showed that pupils' knowledge of plants growing along school paths can positively contribute to an acknowledgement of biodiversity. With respect to socio-scientific issues, van Weelie (2002) has emphasised that biodiversity has the potential to link science and society, because it practises the handling of uncertain information. In support of the above, Dreyfus et al. (1999) have characterised biodiversity as a post-modern theme for environmental education, one that can be helpful for teaching children about the nature of complex real-world problems. Regarding conservation issues, Grace and Ratcliffe (2002) found that the integration of biological concepts with values is a challenge for fifteen to sixteen year-old pupils and, thus, for environmental education. Besides studies on pupils' perspectives on biodiversity issues, Gayford (2000) examined British teachers' perspectives and reported teachers' difficulties to deal with social, political, and economic implications of and causes for biodiversity loss. Lastly, Menzel \& Bögeholz (2006) conducted a qualitative investigation of twelve seventeen to eighteen year-old German pupils' perspectives on biodiversity and found that a consideration of social drivers for and consequences of biodiversity loss was challenging for most pupils. In all, regarding biodiversity, little is known about cognitive frameworks of pupils in an intercultural perspective. Therefore, this study investigated perspectives of pupils who live at a biodiversity hotspot and pupils who live in an industrial country with a medium level of biodiversity.

\section{Cognitive frameworks as learning prerequisites for biodiversity}

According to constructivism, humans are seen as active and subjective constructors of their own knowledge (e.g. Carey, 1985; Caravita \& Halldén, 1994; Piaget, 1974; von Glasersfeld, 2005). During such construction processes, learners perceive and actively process information by either accommodating - adapting their own cognitive structures - or assimilating information in order to make it fit into the current worldview (c.f. Piaget, 1974). With respect to the former, prior knowledge is usually derived from pupils' everyday-life experiences (Posner et al., 1982), and often serves as the basis upon which an individual's understanding of situations within the normal course of life is gained. However, if pupils struggle to gain a deeper understanding when confronted with a scientific topic, underlying cognitive frameworks might be inadequate. Moreover, it is often difficult to alter pupils' existing conceptual structures due to their tendency to be resistant to change. Therefore, 
pupils sometimes retreat to previously held theories, once a particular class module or topic has been completed (Vosniadou, 1992). In this regard, prior knowledge can also be an obstacle within a learning process, especially when previous conceptual structures are opposed to a scientific concept. Therefore, for teaching processes, it is helpful to be aware of pupils' prior cognitive frameworks (Duit \& Treagust, 2003). If teachers show such awareness, learning interventions can actively address cognitive frameworks and consequently foster the learning process. One possibility is to provoke cognitive conflicts through information that contradicts existing cognitive frameworks (Driver \& Erickson 1983; Posner et al. 1982). Also, previous perspectives can be actively included in teaching, e.g. through analogy and metaphor (Scott, Asoko \& Driver, 1991).

Within a learner, cognitive frameworks differ in their complexity. According to Groeben et al. (1988), cognitive frameworks can be represented on at least four different levels namely, associations, concepts, and subjective theories. Associations are the least complex level of a cognitive framework. This paper conforms to Strube's (1984) definition of associations, who defines associations as spontaneously manifested links between terms and broad ideas. Moreover, associations are not exclusive activities that are resigned to memory alone, but are moderated by strategic processes. Due to this fact, associations can shed light on more complex cognitive structures such as concepts or subjective theories. Concepts precede subjective theories regarding their complexity. Albeit their larger complexity, concepts do not usually serve as explanatory elements for complex life situations that span numerous contexts. Subjective theories on the other hand, do. Like scientific theories, they serve as explanatory means and, thus, have a constitutional function for reality. Subjective theories are stable cognitive structures and are often implicit and therefore unconscious to the learner. Less complex cognitive structures such as associations and concepts are often based on underlying subjective theories. In all, a learner's existing cognitive frameworks can be helpful - or challenging - for learning processes. Argumentation strategies can embody underlying cognitive frameworks on the different levels of associations, concepts and subjective theories (Groeben et al., 1988). A thorough and deep analysis of argumentation structures can thus be useful for investigating pupils' cognitive frameworks. Therefore, an exploration of these frameworks can contribute to detecting pupils' learning preconditions, which is important information for designing effective teaching strategies (Duit \& Treagust, 2003). 


\subsection{Research questions}

This study explored German and Chilean pupils' cognitive frameworks on the ideas of biodiversity, its distribution, endangerment, and value. Furthermore, in the perspective of sustainable development, the authors were interested whether pupils' cognitive frameworks supported an acknowledgement of ecological, economic, and social aspects. Therefore, resource dilemmas were used as a model for biodiversity loss. Lastly, the authors were interested whether any structure emerged from the data with respect to pupils' argumentations. To detect prominent argumentation structures, a typology can be a helpful guide. Hence, our research questions were as follows:

1. What associations, concepts and subjective theories can be identified among German and Chilean pupils concerning the terms "biodiversity" and "biological diversity"2 and the distribution, loss, and value of biodiversity?

2. a) Do some pupils emphasise ecological arguments to explain resource dilemmas?

b) Are economic and social facets recognized in a well-balanced way?

3. What typology can be identified within the German and the Chilean sample based on argumentative strategies to explain resource dilemmas?

4. According to the three pillars of sustainable development, is it easier for Chilean pupils to identify social and economic drivers for the loss of biodiversity due to their proximity to a hotspot?

\subsection{Methods}

Regarding biodiversity education, little literature is available on pupils' cognitive frameworks so far. Therefore, the authors decided to conduct interviews that would provide enough time to discuss the topic in a certain depth. An exploratory study on cognitive frameworks needs to provide time to the test persons to enable them to elaborate their thoughts. Interviews have the potential to provide this time. Furthermore, the data basis collected with interviews is larger than for instance data collected with questionnaires, because regarding contents, more can be said than written within a certain time span. In addition to these advantages, interviews are the more suitable method for data collection if

\footnotetext{
2 The synonym terms "biodiversity" and "biological diversity" exist analogously in German and Spanish. The translations are "Biodiversität" and "biologische Vielfalt" in German and "biodiversidad" and "diversidad biológica" in Spanish respectively.
} 
associations should be detected. Written responses are usually better thought out. Spontaneous associations might therefore not be documented in written responses.

Unfortunately, such an approach takes its toll with respect to sample sizes. We therefore limited the sample to 12 pupils per country to be able to provide enough time for in-depth interviews. Thus, the sample consisted of 24 pupils altogether, twelve of them Chilean, twelve of them German.

Given that the topic of biodiversity is complex and also covers ethical issues (such as the judgement of the role of beneficiaries in resource dilemmas), we wanted to ensure that our sample consisted of pupils who were able to gain access to these complex issues within the interview. Therefore, senior pupils between the ages of sixteen and eighteen were selected, all enrolled in the first year of secondary education.

In both countries, we requested the respective schools' headmasters to select four pupils per school based on the following criteria: 1) the chosen pupils' levels of achievement should be representative of both, successful and less successful learners, 2) none of the selected pupils should be involved in any extensive commitment to environmental protection so as to avoid an inclusion of extreme examples within the sample and 3) the pupils should not be informed about the topic or the relation of the interviews to science education research. Moreover, in order to ensure a diverse and representative sample as presented in tables $7.1 \mathrm{a}$ and $7.1 \mathrm{~b}$, we interviewed pupils from different regions and school types in Germany and different school types in Chile respectively. Following this principle, four of the German pupils within the sample lived in rural areas and eight in urban areas. Four of the pupils from the urban area attended a regular secondary school (“Gymnasium”), while four attended a mixed-level school ("Gesamtschule”) in a district, which was characterised by social difficulties.

Within the Chilean sample, four pupils attended an exclusive (and expensive) private school, four a school free of charge in a suburb with a weak socio-economic background, and four attended a private school with medium tuition fees.

By applying the above-mentioned criteria, our sample was relatively diverse but still we acknowledge that it was not representative of an extreme group. Nevertheless, although a certain variation within the sample was achieved by way of the selection criteria, socioeconomic variables were not interpreted systematically. An investigation of interrelations of socio-economic variables and pupils' cognitive frameworks was not an overall aim of this study. Generally, one should not ignore the fact that all pupils interviewed were enrolled in secondary education. As a consequence, the sample consisted of adolescents who all had exclusive access to secondary education and therefore, a certain bias in this respect could not be avoided. 
Table 7.1a. German Sample used in the interviews $(\mathrm{n}=12)$.

\begin{tabular}{|c|c|c|c|}
\hline $\begin{array}{l}\text { Name } \\
\text { (modified) }\end{array}$ & Sex & Type of School & Age \\
\hline Sebastian & $M$ & \multirow{4}{*}{$\begin{array}{l}\text { Grammar School } \\
\text { (Gymnasium") } \\
\text { Semi-urban }\end{array}$} & 17 \\
\hline Matthias & $M$ & & 18 \\
\hline Katja & $\mathrm{F}$ & & 17 \\
\hline Johanna & $\mathrm{F}$ & & 17 \\
\hline Natascha & $F$ & \multirow{4}{*}{$\begin{array}{l}\text { Comprehensive } \\
\text { School } \\
\text { (Gesamtschule") } \\
\text { Urban }\end{array}$} & 18 \\
\hline Julia & $\mathrm{F}$ & & 17 \\
\hline Anna & $F$ & & 17 \\
\hline Ayshe & $F$ & & 17 \\
\hline Simone & $\mathrm{F}$ & \multirow{4}{*}{$\begin{array}{l}\text { Grammar School } \\
\text { (Gymnasium") } \\
\text { Rural }\end{array}$} & 18 \\
\hline Lilli & $F$ & & 18 \\
\hline Olaf & $M$ & & 17 \\
\hline Andreas & $M$ & & 17 \\
\hline
\end{tabular}

Table 7.1b. Chilean Sample used in the interviews $(\mathrm{n}=12)$.

\begin{tabular}{|c|c|c|c|}
\hline $\begin{array}{l}\text { Name } \\
\text { modified) }\end{array}$ & Sex & Type of School & Age \\
\hline Ignacio & $M$ & \multirow{4}{*}{$\begin{array}{l}\text { State school } \\
\text { (public) }\end{array}$} & 16 \\
\hline Elena & $F$ & & 16 \\
\hline Pablo & $M$ & & 17 \\
\hline Paula & $F$ & & 17 \\
\hline Maria & $F$ & \multirow{4}{*}{ Semi-public school } & 16 \\
\hline Albáro & $M$ & & 16 \\
\hline Gustavo & $M$ & & 16 \\
\hline Valería & $F$ & & 16 \\
\hline Igor & M & \multirow{4}{*}{ Private school } & 16 \\
\hline Rosa & $F$ & & 17 \\
\hline Carlos & M & & 17 \\
\hline Alma & $F$ & & 17 \\
\hline
\end{tabular}

The duration of the one-to-one interviews differed between individuals and each interview ranged between 35 and 72 minutes. The interviews were conducted the pupil's mother tongue in each respective school, divorced from science classes. For the purpose of this paper, citations were translated into English from German or Spanish originals by the authors. The in-depth interviews followed a "problem-centred interviews" approach (Witzel, 2000). Therefore, the interviews were structured by a written protocol permitting additional questions and comments when the interviewer felt necessary. The interview protocol consisted of 15 semi-structured questions, mostly with one to three corresponding follow-on questions. The questions followed a basic three-step-structure with a declining number of supporting examples, thus, demanding greater independent thinking from the pupils as the interview progressed (see table 7.2).

As a part of the first step, general questions were posed about the terms "biodiversity" and its synonym "biological diversity", distribution of biodiversity in the world, values, and threats to it.

As a first problem-solving task within the interview, several products, such as teas, phytotherapeutical and highly processed remedies from plant extracts (such as pills and capsules) were presented to find out whether pupils spontaneously linked consumer goods to biodiversity (see figure 7.1). 
Table 7.2. Three-step structure of the interview protocol used in the study.

\begin{tabular}{|c|c|c|}
\hline Step & Content & Examples of interview Questions \\
\hline 1 & $\begin{array}{l}\text { a) General questions about threats for } \\
\text { biodiversity } \\
\text { b) Example: The Devil's Claw } \\
\text { (with detailed interventions, i.e. comments } \\
\text { on e.g. the plant collectors' economic } \\
\text { situation) }\end{array}$ & $\begin{array}{l}\text { What do you associate with the term } \\
\text { "biodiversity"? What does it mean for a place } \\
\text { to contain high biodiversity? } \\
\text { Who do you think should be made } \\
\text { responsible for the loss of the plant } \\
\text { [Devil's Claw]? } \\
\text { Why do you think should people still collect } \\
\text { and sell plants when the plant is threatened? }\end{array}$ \\
\hline 2 & $\begin{array}{l}\text { Transfer example: Boldo } \\
\text { (without interventions) }\end{array}$ & $\begin{array}{l}\text { Let's talk about Boldo leaves. They are } \\
\text { collected from the wild. What are some of } \\
\text { the problems that you think concern the } \\
\text { extraction, use, and trade of this plant? }\end{array}$ \\
\hline 3 & $\begin{array}{l}\text { Questions about } \\
\text { examples and solutions for biodiversity } \\
\text { loss and resource dilemmas }\end{array}$ & $\begin{array}{c}\text { Do you know of any examples where the } \\
\text { trade of particular species (plants or } \\
\text { animals) has reduced their natural livestock? } \\
\text { Do you know any basic approaches for } \\
\text { solving this problem? }\end{array}$ \\
\hline
\end{tabular}

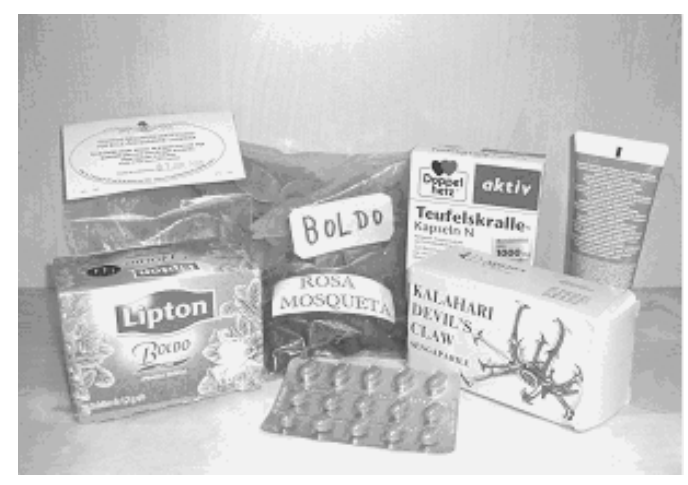

Figure 7.1. Intervention 1: Products presented to the interviewee during the first step of the interview.

A second intervention within the first interview step was solely posed orally. Using the example of the medical plant "Devil's Claw", resource dilemmas were discussed and the following information was given to each interviewee prior to the first question concerning the dilemma:

“An increasing demand for Devil's Claw pharmaceuticals, which serve as a remedy against rheumatism and arthritis, has recently led to a serious decline of the plant in its natural 
habitat, the Kalahari desert. In order to satisfy the industrial demand mostly emanating from Europe and North America, collections of the plant from the wild are frequent. However, a ban on collections would stop plant collectors, who are often living in poverty, earning an income.”

Follow-on questions actively aimed at highlighting economic and social aspects of the resource dilemma. For example, pupils were asked to consider the plant collectors' life situation and explain their thoughts.

Similar features of the above dilemma can be found in the context of the medical plant Boldo (Peumus boldus), native to South America. Due to excessive trading of the plant on Latin American markets, its natural appearance is declining (Vogel, Doll, Muñoz, Razmilic, San Martin \& Vizcarra, 1998). The example of Boldo formed the second step of the interview. In this instance, the dilemma was not explained to the pupil in detail, it was only mentioned in one introductory sentence as presented in table 7.2, step 2. Within this second step of the interview, the research interest focussed on investigating pupils' ability to transfer elements of the first example, the Devil's Claw resource dilemma, onto the second Boldo example. This approach was born out of the hypothesis that the way pupils transferred their knowledge of the resource dilemma to the new situation could shed light on the stability of the pupil's cognitive structure.

In the third step of the interview, pupils were asked to develop possible solutions for resource dilemmas. Similarly to the second step, no assistance was offered in order to stimulate pupils to integrate ecologic, economic, and social aspects with respect to the plant collectors' real life situations. In addition, no examples were given to demonstrate such dilemmas. Given this approach, the third step was regarded as the most abstract step within the interview, one that demanded the greatest amount of independent thinking from the pupil.

\section{Data analysis}

All interviews were fully transcribed, including tacit and non-verbal expressions, such as laughing, hesitation, or pauses. The authors then transformed the texts within a second step. Here, the transcripts were transformed into "edited declarations" ("redigierte Aussagen”, Gropengießer, 2001) where the objective was to prepare grammatically correct language. An interpretation of text material was not yet conducted within this step.

The actual data analysis consisted of three analytical steps, in which we conducted deductive analyses under a particular focus. The first analytical step was a deductive analysis of the entire textual material (Miles \& Huberman, 1994). This step aimed at exploring pupils' 
cognitive frameworks relevant to biodiversity, its value, distribution, and threats to it. Within this first analytical step we also coded responses according to ecological, economic, and social facets and other elements of resource dilemmas such as perspectives on the role of plant collectors.

Within the second analytical step, pupils' cognitive frameworks were then, when possible, assigned to the level of associations, concepts, or subjective theories. A main criterion for an assignment to the groups of associations, concepts or subjective theories was the stability of the displayed cognitive framework. For example, a spontaneous thought that was uttered after a particular question and which the pupils did not explain further, was considered an association. Challenging was a clear allocation to the category of concepts, as no clear definition is available for the category. We therefore decided to consider such frameworks that were repeatedly considered, but which did not lead to an explanation of a circumstance, as concepts. Subjective theories are more clearly defined in that they span numerous contexts and serve as an explanatory basis for phenomena across contexts (Groeben et al., 1988). Therefore, we considered such cognitive frameworks as subjective theories that emerged repetitively during the interview, across the examples applied.

Based on the subjective theories identified within deductive analysis, we created a typology as a third step of the data analysis. For a typological analysis, certain criteria have to be chosen to form the basis for the typology (Kluge, 2000). According to the subjective theories identified in the first step and what we discovered in an earlier analysis with a German sample (Menzel \& Bögeholz, 2006), we constructed the typology based on individuals' consideration of i) ecological, ii) economic and iii) social facets of resource dilemmas. The textual material was then analysed deductively for the three criteria defined (after Kluge, 2000). Eventually, the resulting typology was validated by searching for more parallels between the individuals of one type, besides the underlying typological criteria.

Interrater-reliability was pursued by engaging in check coding as described by Miles \& Huberman (1994) in which coding is conducted by at least two independent researchers for all steps of data analysis. Disagreements were discussed and respective text passages recoded in consensus. The final interrater-reliability was calculated to be $90 \%$ across all categories and was considered to be a satisfactory value. Unfortunately, one interview had to be excluded from the typological analysis due to its poor quality. The respective Chilean pupil, Valería, did not respond to most of the questions and seldom expressed her thoughts in full sentences. Therefore, her cognitive frameworks could not be discovered as the pupil provided too little information. 


\subsection{Results}

\section{Terminology pertaining to biodiversity}

The term "biodiversity" (Biodiversität) was unknown to all the German pupils interviewed. Therefore, in all cases, the term biodiversity was explained to the interviewee before continuing with the interview. In contrast, Chilean pupils correctly interpreted the term "biodiversity" (biodiversidad) as identical in meaning to "biological diversity" (diversidad biológica). In contrast to the term "biodiversity", its synonym, "biological diversity" ("biologische Vielfalt" and "diversidad biológica” respectively) was known across both samples. Both the Chilean and German pupils mostly attributed this term to the variety of plants and animals. However, although less frequently, ecosystems were also regularly associated with biological diversity in both subsamples. The statement of Katja who suggested the following provided an example of such an association: "I would say that $[. .]$. region [rich in biodiversity] has to be relatively multifaceted. There have to be forests for certain animals. There have to be open spaces as well, for bigger animals or other animals [...].” $(95-98)^{3}$

In contrast to the biodiversity levels of species and ecosystems, the consideration of the genetic level of biodiversity appeared only in the case of two German students. For example, consider the following statement generated by Katja: "If they [the animals] were all the same, [...] totally alike concerning the genes [...] they would probably die out easier, I guess. It's because then only one thing has to happen the animal can't get along with [...]. This would, then, affect the entire species.” (129-134) In this example, the pupil's thoughts exceeded the relatively low complexity of associations. Katja put her thoughts into a meaningful context in that she considered possible negative consequences of genetic uniformity. Her comment should thus be considered as a concept.

In both subsamples, a prominent misinterpretation of the terms "biological diversity" and "biodiversity" was an interpretation as diversity of the subject biology, thus, the different biological disciplines. This interpretation often occurred as an association, but tended to be stable in some cases, which is then rather to be interpreted as a concept.

\section{Distribution of biodiversity}

Within the German sample, pupils directly interpreted tropical forests or wilderness areas in Africa and Latin America as being indicative of regions high in biodiversity. Although this

\footnotetext{
${ }^{3}$ The numbers in brackets indicate the line numbers in the original transcript.
} 
assumption is correct, it is interesting to note why these areas were viewed as being extraordinarily rich in biodiversity. In this regard, a popular concept concerning the lack of space in industrial countries could be identified, which seemed to be influential when pupils spoke about the distribution of biodiversity. Within this concept, pupils assumed cramped cities with practically no space for plants and animals as regions of extremely low biodiversity. Although much more popular among German pupils, the concept was also identified within the Chilean sample. For instance, the Chilean pupil Albáro stated: “[...] I think that poor countries have higher biodiversity because they don't have industry, they don't have anything, it's [still] all here." (729-730, 734) Besides the concept of space, a popular strategy used by pupils to explain lower biodiversity in certain regions was through the concept of climatic characteristics. Here, deserts and Polar Regions were frequently seen as regions of low biodiversity. The concept upon which these associations were built seemed to be one that was based on the contention that extreme climatic conditions oppose high biodiversity. Accordingly, one German pupil, Johanna, explained the relatively low biodiversity in Germany as well as in "warmer" countries by means of the following reasoning: "In Germany, it could be that the climate is bad here, there is too little sun [and it is] always too wet, so the plants die. [...] And in warmer countries, plants don't have the time to grow." (366-371) In what is probably linked to the same concept, Europe was mentioned as a region of high biodiversity in some cases. For instance, the German adolescent Simone stated: „[Areas of high biodiversity] are more likely to be found here in Europe because the weather is not extremely hot nor extremely cold, it's [a much larger] spectrum, [suitable] for many living organisms.” (139-141) This example served to support the concept that extreme climatic conditions could in some way or other be harmful for biodiversity.

\section{Loss of biodiversity}

Besides the already mentioned concepts of extreme climatic conditions and the lack of space, few arguments were spontaneously given to explain the loss of biodiversity. Before the first dilemma example had been mentioned (see table 7.2), only one pupil in the entire sample, the German pupil Lilli, took social reasons for biodiversity loss spontaneously into account. Thereto, Lilli issued the following statement: "I think of the tropical rainforest [...]. There, it could be difficult to stem desertification [...] as pastures are expanding more and more. [Sometimes] nomads are driven away to the south to let their cattle graze there. But it is difficult to do something about this.” (284-289) Regarding threatened biodiversity, Chilean pupils associated numerous local examples, such as the Huemul (an endemic Chilean deer species), the Camote (a Chilean wolf species), or Araucariae (Chilean endemic tree species) 
to examples. Within the German subsample, only Sebastian mentioned one example of a threatened plant that also exists in Germany, i.e. Edelweiss, an endemic species to the Alpes region. However, international examples, such as whales or elephants, generally prevailed in the German sample.

\section{The value of biodiversity}

Within both samples, the value of biodiversity was frequently associated with its value as a food and medicine source. An economic value of biological diversity, defined as the possibility to establish a market with biological goods, was identified as a concept in both samples although it was slightly more prominent among Chilean pupils. For example Carlos, a Chilean pupil, expressed a concept of economic value in that he considered the utilisation of local plant and animal species to be not only of cultural interest but also as a tourist attraction. A concept of aesthetical value of biodiversity was identified among several German pupils (e.g. the beauty of flowers and trees), but rarely occurred within the Chilean sample. In contrast, a very interesting concept that defined biodiversity as a requirement to survive ecological changes was identified within both subsamples. The Chilean pupil Albáro provided a datum, which supports this opinion: "Well, [biological diversity is important] for everybody [...]. It's like history. If one considers the principle of animals, how they live and the changes that they had [to go through] and how they succeeded to pass all these things." (212-216) The aforementioned statement of the German pupil Katja that described a genetic variety as an element of biodiversity, suggested a similar direction in terms of the ability of species to survive changes due to their genetic flexibility. Therefore, Carlos and Katja obviously interpreted a high genetic diversity as an insurance of survival in case of changes in the environment.

Overall, the value of species for the respective ecosystem to which they belong, value for humans when using biological diversity for the production of basic commodities such as clothes, and a possible value for science and research were prominently supplied as examples in both samples.

\section{Subjective theories about the loss of biodiversity}

According to the three pillars of sustainable development, the loss of biodiversity and especially the threat of biodiversity due to resource dilemmas can only be understood in depth if pupils connect economic factors to ecological and social factors. During our data analysis, it emerged that influential subjective theories that contained either a strong ecologic, economic, or social stance, influenced pupils' judgements on the overall 
biodiversity problem and within the context of the dilemma examples that were provided. In this regard, three subjective theories emerged across the sample, namely, loss of biodiversity due to ecological reasons, loss of biodiversity due to social reasons and loss of biodiversity due to economic reasons.

\section{Subjective theory 1: Loss of biodiversity due to ecological reasons}

The subjective theory "Loss of biodiversity due to ecological reasons" is characterized by a view that excluded social and economic factors. For example, such a subjective theory was exemplified by the profile of the German pupil Natascha. Throughout the entire interview, ecological drivers (or what Natascha considered to be drivers for biodiversity loss) came to the fore strongly. Such thinking was shown in her explanation of the loss of Devil's Claw where she emphasised ecological arguments, such as soil conditions or the plants' lack of an ability to recover: "There are some plants [that] don't grow again, once they have been cleared or something [like that]. (647-648) Maybe after a while, [...] nothing grows again. That depends on the soil or the weather.” (723-724) During the Boldo transfer example, her arguments were again strongly influenced by an ecologically orientated subjective theory: “[...] I don't believe that the soil is always producing something, nutrients for example. It's also possible that nutrients get destroyed at a certain point and don't recover. When continuing like that, there'll be the same problem as with the Devil's Claw.” (947-950)

When Natascha suggested possible solutions for resource dilemmas, she briefly considered the protection of wild growing plants. Nevertheless, her strong emphasis on ecological factors finally prevailed. This emphasis eventually prevented her from finding a realistic solution for biodiversity loss, which was exemplified by the following quote: "[What we can do] depends on the humans. To pay attention to nature, maybe to grow something. However, it depends on the climate after all." (1203-1204) Due to Natascha's narrow view of the problem as demonstrated above, her particular subjective theory did not allow for empathy with plant collectors. What is more, her tendency to overlook socio-economic implications had serious consequences for accurately judging the participants' role in the resource dilemmas, in that she blamed the plant collectors for the destruction of the resource. In this respect, Natascha judged the behaviour of the plant collectors in the case of Boldo as follows: "[I would call the behaviour of the individual participants in the case of Boldo] selfish. Maybe [they] should sometimes think of other people as well. [...] if people go on like that, we soon won't have nature any more, nothing." (955-957) 
Subjective theory 2: Loss of biodiversity due to economic reasons

Besides the subjective theory "loss of biodiversity due to ecological reasons", a second subjective theory emerged from the data. Here, the focus was on economic arguments, such as an increasing demand for Devil's Claw products as a reason for pressure on the respective natural resource. For instance, even though the Chilean pupil Elena considered ecological and economic forces to be most threatening to Devil's Claw, the focus of her argumentation was on an economic driver. For example, consider the following statement obtained from Elena: “[I think the peoples' interest in collecting the plant] is money [...]. Some pharmaceutical enterprises might preserve [the plants], [in order] to be able to sell products. However, they [still] go on producing, possibly without any conscience [...]. [This might happen] to satisfy their needs, but in the end, it's all for the money." (294-296, 300301) With respect to Elena's position, the situation pertaining to Boldo was similarly sketched: "[I would judge the behaviour of the persons participating in the process] ambitious or maybe that they only care for mass production and selling, and not [for the fact that] they do harm to nature or to the ecosystem where they take the things from." (426-428) What is of particular interest in the case of Elena, are the solutions that she developed during the third phase of the interview. Elena assumed that people would only protect the Alerce, a Chilean endemic tree, once the tree's economic value is recognized. According to this strong emphasis on economic interest as a means for protection, Elena also considered the possible disadvantages to such species that do not show an economic value, provided by the following datum: "[...] for example, if they [the companies] use resources such as the Alerce, [they] will be more protected, because in the end [...] money always makes a difference. So if they use half of [the money available for conservation purposes] for Alerces, there will be less protection, less care for other species to which they assign less money." (547-550)

\section{Subjective theory 3: Loss of biodiversity due to social reasons}

The third subjective theory that was identified was "Loss of biodiversity due to social reasons". Here, social aspects of resource dilemmas emerged as the core of the subjective theory and were considered continuously throughout the entire interview. In this theory, economic aspects played a certain role but were usually only considered within social foci. To demonstrate the nature of this theory, the profile of Katja (German) will be examined. Katja described the example of Devil's Claw as follows: "I think [the situation is so complicated because] the people harvesting it [the plant], somehow need that money, otherwise they wouldn't have a means of subsistence. And maybe they don't have the chance 
to do something else and that they really need the money [makes the situation so difficult]." (398-401) When talking about Boldo, Katja again focussed her arguments on the plant harvesters' socio-economic situation, in this case on their need for income as demonstrated by the following: "I would suggest that [concerning the main participants of the trade with Boldo] again, there are plant collectors receiving a salary for it [the plant] which is then exported to us.“ (463-465) According to the underlying subjective theory, Katja's solutions were also aimed at addressing the social and economic situation, which is supported by the following: „One has to offer other possibilities to the people to be able to earn money.” (412, 416) Later during the same interview, Katja considered a prevention of trade of Boldo as a possible solution, but then strongly emphasised social aspects again as shown below: "[...] it would be over for them [the collectors] as well. If there is no demand, they wouldn't earn any money.” (489-490) Altogether, Katja used ecological, economic, and social arguments through the interview. Whenever she talked about the loss of biodiversity, social considerations came forward. The underlying subjective theory "loss of biodiversity due to social reasons” showed to be a strong underlying, structuring element in the case of Katja.

\section{Typology}

Subjective theories serve as a structuring element for phenomena across different contexts (Groeben et al., 1988). In our study, the three identified subjective theories were prominent in both the German and the Chilean subsample. This observation led to the hypothesis that a typology of pupils could emerge from the data. We therefore examined the textual material systematically during a deductive analysis. However, in order to set up a typology based on deductive analysis, criteria have to be defined (Kluge, 2000). As the three subjective theories, which we identified within our sample, apparently focussed on one sphere of sustainable development each, we decided to choose ecological, economical, and social arguments as the core typological criterion. The underlying criteria for our typology are presented in detail in table 7.3. All pupils (except the Chilean pupil Valería) could be assigned to one of the three types we identified namely, the ecological type, the balanced type and the economical type. 
Table 7.3. Typology, criteria and pupils’ typological affiliation.

\begin{tabular}{|c|c|c|c|c|}
\hline Type & $\begin{array}{l}\text { Subjective } \\
\text { Theory }\end{array}$ & Criteria & $\begin{array}{l}\text { German } \\
\text { participants }\end{array}$ & $\begin{array}{l}\text { Chilean } \\
\text { participants }\end{array}$ \\
\hline \multirow{6}{*}{$\begin{array}{l}\text { Ecological } \\
\text { Type }\end{array}$} & \multirow{6}{*}{$\begin{array}{l}\text { Loss of } \\
\text { biodiversity } \\
\text { due to } \\
\text { ecological } \\
\text { reasons }\end{array}$} & \multirow{6}{*}{$\begin{array}{l}\text { Ecological arguments clearly } \\
\text { dominant in at least two stages of } \\
\text { the interview } \\
\text { - Subgroup I: Transfer example } \\
\text { Boldo is not recognized by some } \\
\text { Chilean pupils } \\
\text { - Subgroup II: Ecological arguments } \\
\text { present in all three stages of the } \\
\text { interview } \\
\text { - Subgroup III: Despite a clear } \\
\text { ecological focus, social solutions } \\
\text { are suggested }\end{array}$} & \multicolumn{2}{|l|}{ Subgroup I: } \\
\hline & & & & $\begin{array}{c}\text { Albáro, Alma, } \\
\text { Gustavo, Pablo, } \\
\text { Paula } \\
\end{array}$ \\
\hline & & & \multicolumn{2}{|l|}{ Subgroup II: } \\
\hline & & & $\begin{array}{c}\text { Natascha, } \\
\text { Ayshe }\end{array}$ & $\begin{array}{l}\text { Rosa, Igor, } \\
\text { Ignacio }\end{array}$ \\
\hline & & & \multicolumn{2}{|l|}{ Subgroup III: } \\
\hline & & & $\begin{array}{c}\text { Johanna, Anna, } \\
\text { Matthias }\end{array}$ & \\
\hline $\begin{array}{l}\text { Economic } \\
\text { Type }\end{array}$ & $\begin{array}{l}\text { Loss of } \\
\text { biodiversity } \\
\text { due to } \\
\text { economic } \\
\text { reasons }\end{array}$ & $\begin{array}{l}\text { Concentration on economic } \\
\text { arguments in the first stage of the } \\
\text { interview } \\
\text { - Presence of economic arguments } \\
\text { in the Boldo transfer example }\end{array}$ & Andreas & Elena, Maria \\
\hline $\begin{array}{l}\text { Social } \\
\text { Type }\end{array}$ & $\begin{array}{l}\text { Loss of } \\
\text { biodiversity } \\
\text { due to social } \\
\text { reasons }\end{array}$ & $\begin{array}{l}\text { Social arguments present in all three } \\
\text { stages of the interview } \\
\text { - No clear dominance of either type } \\
\text { of argument }\end{array}$ & \begin{tabular}{|} 
Simone, Lilli, \\
Katja, \\
Sebastian, Olaf, \\
Julia
\end{tabular} & Carlos \\
\hline
\end{tabular}

The ecological type

The main criterion for an assignment to the ecological type was a clear dominance of ecological arguments throughout the interview and an emphasis on ecological arguments in at least two steps of the interview. Two steps were chosen as a minimum requirement to qualify for the ecological type, as the interview consisted of three steps altogether. Therefore, an emphasis on ecological arguments in two steps of the interview represents an overall emphasis on such arguments within the respective interview. An overview on the presence of ecological, economical and social arguments within the three steps of the interview is depicted in figure 7.2. According to the above-mentioned criteria, thirteen pupils were assigned to the ecological type. However, some differences in the argumentative structure could be identified among the participants. As a consequence, the ecological type was divided into three subgroups (see figure 7.2). 


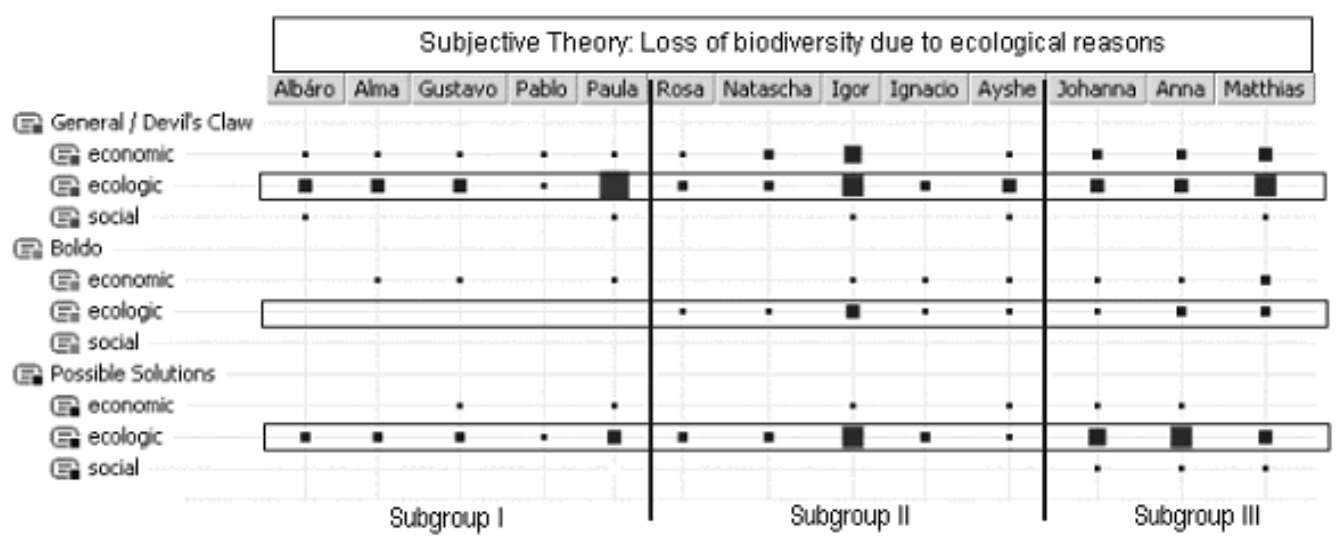

Figure 7.2. The ecological type. The larger the squares are, the greater the number of arguments pertaining to the respective category of ecological, economical, or social arguments. Steps of the interview: 1) General questions and input example Devil's Claw, 2) Boldo transfer example and 3) possible solutions suggested by the pupils. Ecological arguments are highlighted by the rectangles.

As was the case in all the subgroups, group I fulfilled the main criterion of the ecological type, a dominance of ecological arguments in at least two steps of the interview. In contrast with the other pupils of the ecological type, the pupils of this group did apparently not consider the Boldo transfer example as a problem. If so, arguments only occurred as concerns about a possible market value of the plant (included under "economic" arguments). Due to this argumentative structure, Boldo was not interpreted as a problem of possible decline in biodiversity, let alone as a socially rooted problem. According to the main criterion for the ecological type, solutions within the third step of the interview were focussed on ecological conditions, such as an enhancement of soil conditions. Interestingly, none of the adolescents of subgroup I mentioned social solutions, for example one that would consider the plant collectors' social situation. Regarding the pupils of the ecological type, it is worthwhile to note that subgroup I consists of Chilean pupils exclusively: Albáro, Alma, Gustavo, Pablo, and Paula.

In contrast with the first subgroup (see figure 7.2), the Boldo transfer example was recognized within subgroups II and III. Here, statements about Boldo were prevalent during the second stage of the interview (see figure 7.2, step "Boldo"). However, Boldo was mainly regarded as an ecological problem, just like in the Devil's Claw example. Within the third interview stage, solutions were again focussed on the enhancement of ecological conditions. As the associates of subgroup II considered Boldo, ecological arguments were dominant in all three stages of the interview. Similarly to subgroup I, none of the members of the second subgroup suggested any social solutions. It was found that subgroup II is a mixed group with 
respect to its associates in that it consists of three Chilean (Rosa, Igor, and Ignacio) and two German pupils (Natascha and Ayshe).

Just like subgroup II, subgroup III was characterized by a recognition of both the Devil's Claw and Boldo dilemma, again under a predominant consideration of ecological arguments. Here, the difference with the two before-mentioned subgroups lies in the development of possible solutions within the third interview phase. In contrast to subgroup I and II, social solutions were developed rather than concentrating on ecological solutions. However, social considerations were still overlaid by ecological arguments so that members of subgroup III still form part of the ecological type. Regarding their nationality, all three associates of subgroup III were part of the German sub-sample: Johanna, Anna, and Matthias.

Assuming that the emphasis on ecological facets of biodiversity loss is due to the underlying subjective theory "loss of biodiversity due to ecological reasons", a consideration of the plant collectors' socio-economic situation might be difficult to achieve by the members of all three subgroups of the ecological type. Particularly, social dilemmas cannot be taken into account when an emphasis on ecological threats for the respective plant dominates. Therefore, the authors contemplated whether negative evaluations of the plant collectors' behaviour were prominent within this type. In fact, all associates of the ecological type, except Matthias (German) expressed negative judgements toward plant collectors in a resource dilemma. In particular, the data suggests that plant collectors were at least partially responsible for the loss of the plants within the examples pertaining to the ecological type.

\section{The economical type}

Three pupils made up the economical type, Andreas (German), and two Chilean pupils, Elena and Maria, as presented in figure 7.3 below.

During the first stage of Andreas', Elena's and Maria’s interviews, a clear economic focus became visible, one that emphasised an economic value of Devil's Claw. In addition, all three pupils considered consequences for commercial trade following an elimination of the plant. A similar approach was found in the context of Boldo, which was also frequently discussed as an economic consumable. Even though a large emphasis was placed on an economical aspect, the two Chilean pupils also reflected upon ecological consequences of the loss of Boldo. In particular, the two Chilean pupils considered a disturbance of the ecosystem caused by the loss of the plant. Within the economical type, no clear tendency emerged regarding solutions. For instance, Maria (Chilean) focussed on ecological solutions, while Elena, also Chilean, likewise suggested economic changes and Andreas (German) referred to possible social solutions on one occasion. 


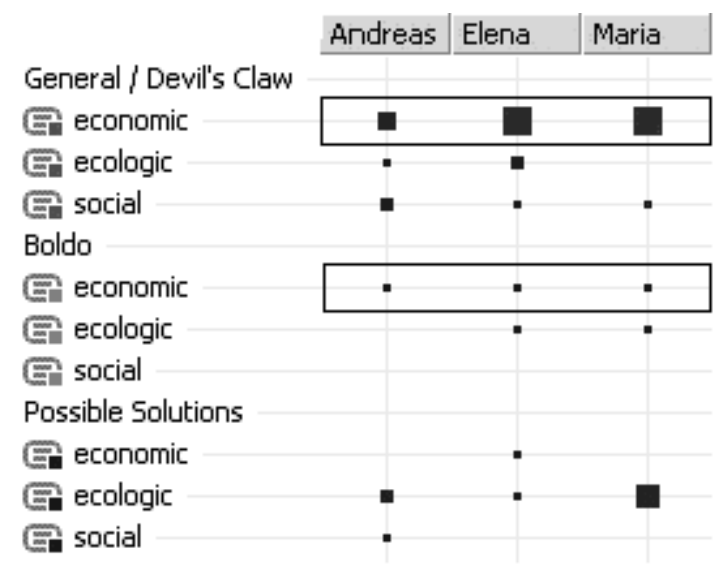

Figure 7.3. The economical type. Predominant arguments are economic within the first part of the interview and are also transferred onto the Boldo transfer example (step 2 of the interview). Economic arguments are highlighted by the rectangles.

If we regard judgements of the plant collectors' role, negative evaluations occurred, especially in the case of Maria (Chilean), who on seven occasions blamed plant collectors for threatening Boldo. Also Andreas (German) blamed plant collectors once, and Elena as well did so, on four occasions.

In all, the economical type was a rather inhomogeneous group. Moreover, in the cases of Andreas and Maria, the subjective theory "loss of biodiversity due to economic reasons" was not clearly identified within the first and the second analytical steps of our study. Rather, economic cognitive frameworks could only be identified on the level of concepts.

\section{The balanced type}

Most of the pupils assigned to this third group used ecological, economic, and social arguments in all three stages of the interview. Thus, a balance of all three types of arguments (ecological, economical, social) occurred within all three steps of the interview. There was only one exception to this finding: Carlos, the only Chilean member of the balanced type, did not mention social solutions and therefore missed social arguments in the third interview step. These results are presented in figure 7.4 below. 


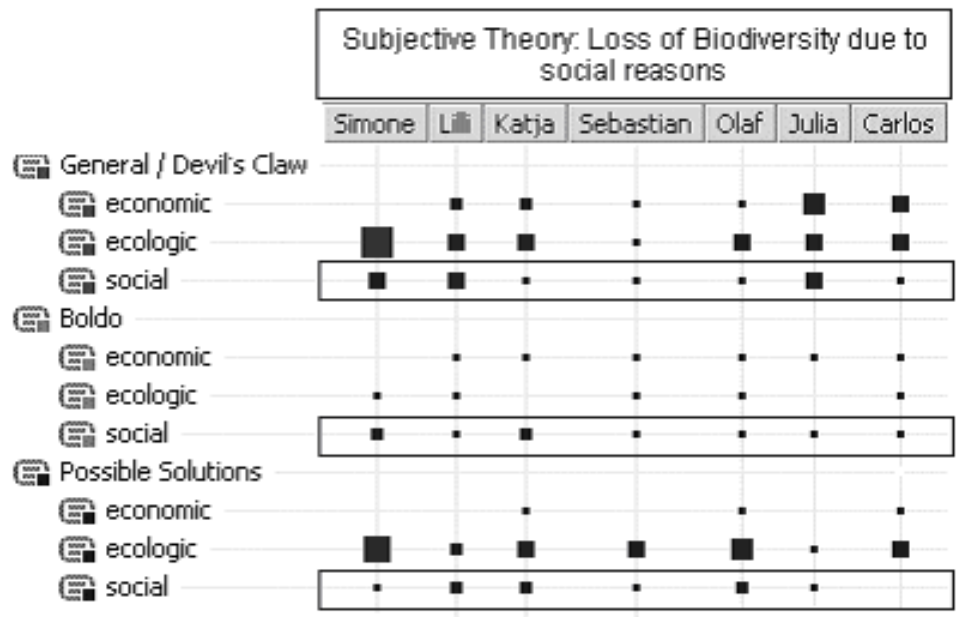

Figure 7.4. The balanced type. Social arguments appear within all three stages of the interview. Social arguments are highlighted by the rectangles.

As a central characteristic of this group no clear dominance of any type of argument (ecological, economic, or social) became apparent in the pupils' argumentations (see figure 7.3). Due to the well-balanced consideration of the three aspects, dilemma situations were adequately considered. This means, pupils of the balanced type were aware of resource dilemmas with regard to the three pillars of sustainable development. Therefore, pupils took the plant collectors' weak economic situation and their social dependence on natural resources into consideration. As a consequence, pupils also considered conflicts in interest between the possible non-sustainable exploitation of a resource on the one hand and a satisfaction of basic economic needs on the other.

Interestingly, negative evaluations of the plant collectors' role occurred less frequently within the balanced type. Only Julia (German), and Carlos (Chilean) describe the plant collectors' role in a negative way. After all, Julia criticised the exploitation of the plant by collectors only once, yet Carlos did so on five occasions. In all, members of the balanced type were pupils who succeeded in a well-balanced consideration of ecological, economic, and social aspects. Therefore, other than pupils of the two types outlined above, members of the balanced type showed no concentration on either type of argument.

\subsection{Discussion}

The results obtained from an analysis of pupils' cognitive frameworks on terminology, distribution, loss, and values of biodiversity suggest that individual differences exist for perceiving the complex issue of "biodiversity". Although highly descriptive, qualitative data 
cannot tell us anything about pupils' views on biodiversity within a larger sample. Nevertheless, this study shows how deep insight into pupils' prevalent cognitive frameworks could be uncovered by pursuing the prominent argumentation paths of individuals. This approach could be used to get an idea into how cognitive frameworks differ between pupils living at biodiversity hotspots and those living in industrial countries.

\section{Terminology}

In contrast with the Chilean pupils, none of the German pupils had ever heard of the German term for "biodiversity". This finding suggests that the term should be introduced into science classes, probably by presenting it as the technical term for "biological diversity" (which is commonly known by German pupils). Furthermore, educators have to aspire towards a deeper understanding of the "biodiversity" concept since the data showed that it was commonly interpreted as a synonym for "variety of species" rather than a consideration of all three types of biodiversity. Nevertheless, pupils seemed to be open towards the biodiversity of ecosystems, which is a good starting point. Moreover, the study suggests that an understanding of the meaning of genetic diversity is probably the biggest challenge for pupils. Although being challenging, the notion of genetic diversity as the engine of evolution and therefore as an "insurance" for survival under changing environmental conditions, was mentioned by two pupils, which serves as a positive example for a basic understanding of genetic variety. Furthermore, an adequate and complete introduction of the terms "biodiversity" and "biological diversity" should also help to overcome the popular misinterpretation of the term "biodiversity" as a research method or as some or other sub discipline of biology.

\section{Distribution of biodiversity}

Within the German sample, pupils accurately associated the tropics and wilderness areas in Africa to regions that are representative of high biodiversity. Similar associations were discovered with respect to Latin America within the Chilean sample. Therefore, we assume that a broad understanding of the distribution of biodiversity seems to exist, although the term itself was unknown to German pupils.

Interestingly, the concept of "lack of space" was found in nearly all the interviews. The concept probably derives from everyday-life experiences obtained from living in, or visiting big towns and cities. One reason that it was more popular among German pupils was probably due to a general negative view of urbanisation. In fact, the destruction of natural habitats is a problem for biodiversity preservation in general (Pullin, 2002). Nevertheless, 
pupils seemed to have a very concrete and practical view on the problem and in doing so, overestimated the role of cities. In contrast, other important threats to biodiversity, such as the defragmentation of habitats or depletion of resources were not spontaneously considered. These results suggest that the concept "lack of space" should be broadened to include the topic of habitat destruction outside big cities. Another approach in teaching practice could be to include urban ecology as a topic. By highlighting a perspective on cities as species' habitat, a cognitive conflict could be achieved. Possibly, the concept would then be broadened in that cities will not be regarded as threats to biodiversity per se.

Upon explaining reasons for low biodiversity, the participants cited prevalent climatic circumstances as common examples. Although this concept was popular, it is possible that it undermines the learning process, because climatic circumstances are not threats to biodiversity per se. For instance, pupils might learn about the fact that biodiversity hotspots are often located in regions where extreme climatic and ecological conditions prevail (e.g. tropical forests with poor soil conditions or deserts containing extraordinary adaptation strategies of plants and animals). In such instances, the concept of low biodiversity due to extreme climatic circumstances may hinder the learning process. To avoid this, the concept of climatic extremes should be made explicit by including examples of highly adapted plant and animal species in biodiversity education.

\section{Loss of biodiversity}

The above-mentioned concepts of "lack of space" and "extreme climatic conditions" also influenced pupils’ perspectives on biodiversity loss. In contrast, socio-economic drivers for biodiversity loss were seldom considered. Only Lilli (German) made the spontaneous association of socio-economic factors with biodiversity loss, thus, considered these factors before they were actively brought up through dilemma examples. In this regard, and especially with respect to the Chilean sample, the researchers had expected a certain intuitive consideration of social problems as aggravators of biodiversity loss. However, our findings suggest that we cannot assume an easier understanding of socio-economic drivers for biodiversity loss due to the fact that adolescents live in close proximity to a biodiversity hotspot. Therefore, this finding contradicts our earlier expectations. Moreover, we can conclude that empathy cannot automatically be assumed when a pupil lives close to a biodiversity hotspot, where resource dilemmas occur.

According to examples for endangered biodiversity, only one German pupil within our sample provided one single local example. In this regard, the data supports numerous reports that suggest that pupils also have a weak taxonomic knowledge in general (e.g. Balmford et 
al., 2002; Bebbington, 2005; Jaekel \& Schaer, 2004). If we bring these findings together with what is reported in literature, we can conclude a low awareness of local species among German pupils in general. Therefore, educational measures in Germany should include describing local examples of biodiversity - and also of biodiversity loss. Moreover, teaching about local biodiversity could not only meet in closing the deficit in taxonomic knowledge, but also enhance a general acknowledgement of the intrinsic value of biodiversity and thus serve as motivational factor towards protecting biodiversity (Lindemann-Matthies, 2002b, 2006).

\section{Value of biodiversity}

Pupils from both countries mentioned the value of biodiversity as a source for food and medicine. These ideas can probably be ascribed to the products presented to the interviewees within the first stage of the interview (see figure 7.1). Howsoever, the numerous references to commercial products show that human benefit from biodiversity seemed to be a plausible idea for pupils in both samples. Thus, pupils' ability to recognise the actual usefulness of biodiversity for human benefit could be a good starting point for devising learning interventions. For example, a reflection on consumer products with plant ingredients that pupils use in their everyday life could be included in teaching interventions. In addition, it should be mentioned that the protection of biodiversity must not be exclusively motivated for human benefit only. A less human-centred approach in the discussion of the value of biodiversity is especially important, as non-monetary benefits such as aesthetic value, were seldom mentioned within the Chilean sample. Interestingly, Albáro (Chilean) and Katja (German) mentioned the importance of biodiversity as an insurance against changes in the environment and therefore rose above a purely human-centred perspective. At the same time, this interesting data demonstrates an understanding of the main nuances of genetic variety and serves as support for the importance of introducing all three layers of biodiversity to pupils.

\section{Subjective theories}

Subjective theory "Loss of biodiversity due to ecological reasons"

The authors suggest that the profile of Natascha (German) provides convincing evidence for how the subjective theory "loss of biodiversity due to ecological reasons" served as the basis for her argumentations within the entire interview. The example of Natascha shows how difficult it is for a learner to go beyond the limits of an existing cognitive framework. According to the underlying subjective theory, the loss of biodiversity was continuously 
explained by virtue of soil conditions, climate, and plants' inability to recover after harvesting, often under the guise of erroneous biological knowledge. Even after an active demonstration of social and economic interrelations, Natascha was unable to integrate these perspectives into her arguments. Most striking was her tendency to accuse local plant collectors for biodiversity loss. In this regard, we interpreted her non-existent socioeconomic perspective as a hindrance for considering the perspective of people involved in a resource dilemma. We suggest that the result was a lack of empathy, when it could be argued that she did not have an understanding for those involved at "grass roots" level. All in all, the complexity of the "biodiversity" topic was not adequately acknowledged in Natascha's interview and its potential as a real-world example in environmental education (Dreyfus et al., 1999) consequently not fully tapped. In particular, Natascha's profile clearly demonstrates that the subjective theory "loss of biodiversity due to ecological reasons" had at least two main negative consequences for the learning process:

1. The application of weak ecological facts whenever "accurate" ecological arguments fail to explain biodiversity loss;

2. The absence of empathy with people in real resource dilemmas due to difficulties in accepting social-economic traps as a cause for biodiversity loss.

\section{Subjective theory: "Loss of biodiversity due to economic reasons"}

Arguments that were focussed on the economic value of biological resources were particularly demonstrated by the profile of Elena (Chilean). Here, monetary value was considered as something positive and a factor that can play a large role in protecting a plant. In contrast however, Elena also described how greed for money could be a reason for the depletion of a resource itself. Therefore, she seemed to be ambivalent about the role that market forces play, but she did regard money as the driving force in the protection or depletion of a resource. Although economic considerations formed the basis for her arguments, the economic situation of plant collectors was not considered and her arguments remained at the more abstract level of market forces. Here again, just as in the example of Natascha, for Elena it was difficult to leave the existing subjective theory behind. As a consequence, Elena remained within the argumentative limits of her existing cognitive framework. In doing so, Elena neither considered the plant collectors' economic situation nor the consumers' role as potential purchasers of a product. Moreover, we suggest that her focus on economic forces hindered her consideration of more realistic solutions. We can therefore conclude that the subjective theory "biodiversity loss due to economic reasons" does not capture a realistic understanding of resource dilemmas. In this regard, her somehow 
naïve assumption that plants of economic value would automatically be protected did not correlate favourably with the actual examples presented to the interviewees. In particular, Elena's profile shows that

1. Economic forces were considered at an abstract level without reflecting upon the role of individuals such as those in resource dilemmas or the consumers themselves,

2. Economic forces can be stereotyped positively or negatively,

3. A consideration of economic forces does not automatically lead to the recognition of sustainable economies as a solution.

Subjective theory: "Loss of biodiversity due to social reasons"

In comparison with Natascha and Elena, Katja (German), applied a different argumentative structure. In contrast to Natascha and Elena, her underlying subjective theory fostered an inclusion of ecological, economic and social perspectives. In this case, from the beginning of the interview, Katja had already included social and economic factors into her considerations even before the interviewer broached the issue of resource dilemmas. For example, she placed a strong emphasis on a need for money as an indirect threat to biodiversity and also reflected the role of traders, exporters, and consumers within the same argument. It is possible that this holistic and more satisfying explanation prevented her from constructing false ecological facts. Additionally, it is striking that Katja never used isolated ecological arguments during the interview and thus suggests that she is able to comprehend both the ethical and factual complexity of the biodiversity concept (Barkmann \& Bögeholz, 2003).

Overall, on the one hand Katja did not succeed in finding a realistic solution to the resource dilemmas, but it was on the other hand her careful thought about the lack of income alternatives that prevented her from suggesting solutions that would eventually worsen the plant collectors' situation. Thus, Katja showed empathy and never blamed the plant collectors directly for depleting the resource. Katja's statements highlight the high potential of the "biodiversity" subject as means for fostering links between science, society and environmental education (e.g. van Weelie, 2002). In general, Katja’s profile suggests that the subjective theory "loss of biodiversity due to social reasons" has the following consequences for the learning process:

1. A consideration of the plant collectors' perspective took place during the discussion of resource dilemmas and therefore, empathy with people in resource dilemmas was imparted; 
2. All three pillars of sustainable development were considered during the discussion of resource dilemmas: Economical, ecological, and social facets, an approach, which is desirable for an appropriate understanding of biodiversity and its loss (Shiva, 2000).

\section{Typology}

Although qualitative data from small samples does not allow for broad generalisations, the study shows a general prevalence of the ecological type across both, the Chilean and the German sample (see table 7.3). At the same time, learners of this type are the least successful in the proper understanding of a resource dilemma. Therefore, an inclusion of socioeconomic aspects of biodiversity loss into biodiversity education is a central challenge. It is interesting that pupils that are assigned to the ecological type tend to negatively judge participants in resource dilemmas. This lack of empathy is alarming for two reasons. First, blank negative judgements of people in difficult economic situations are unfair attributions of responsibility that reflect missing solidarity. Second, an ability to show empathy can at the same time have a positive influence on pro-environmental attitudes and behaviours (Berenguer, 2007). Therefore, biodiversity education should foster the development of empathy. In teaching practice, role-plays could be a starting point in which pupils are encouraged to take the perspective of people in resource dilemmas. For example, groups of pupils in the classroom could represent different stakeholders in a resource dilemma. In this regard, teachers' attitudes might play an essential role in that they must be open towards socio-economic issues. Gayford (2000) has reported that biology teachers have difficulties with interdisciplinary aspects of the "biodiversity" topic. Although his research was confined to Great Britain, it is fair to assume that the same reluctance towards socio-economic issues might exist among biology teachers in other countries. Therefore, the data of the present study supports Gayford's claim in suggesting that appropriate teacher training has to be aspired to overcome teachers' reservations in this respect (Gayford, 2000). In support of the above, Tal and Kedmi (2006) showed that science classes that are less content-based and not value-free enhanced Israelian pupils' argumentation about socio-scientific issues and suggest teachers should shift to a more socio-cultural approach. Our data supports the claim for such a shift and teachers should therefore be supported in this respect.

In contrast to the ecological type, members of the balanced type serve as a positive example for an interdisciplinary perspective to biodiversity that encourages a more complete understanding of resource dilemmas. What's more, respondents within the balanced type showed an ability to empathise with people who are under economic pressure such as plant collectors who face their own resource dilemma. Although quantitative methods with a 
larger sample would be necessary for constructing correlations between arguments, the qualitative data nevertheless suggests a strong relationship between the subjective theory "loss of biodiversity due to social reasons" and participants' ability to show empathy. In this respect, pupils could positively contribute to biodiversity education. For example, pupils that are able to show such empathy could support those classmates who express difficulties to take the perspective of people in resource dilemmas, for example in teamwork tasks. In a class that strictly disciplinary oriented toward ecological contents, pupils who show such empathy might not tap their full potential.

In an earlier study, we showed that the subjective theory "loss of biodiversity due to ecological reasons" and "biodiversity loss due to social reasons" could be identified within the German sample (Menzel \& Bögeholz, 2006). In contrast, in the previous study, the subjective theory "loss of biodiversity due to economic reasons" was not found within the German subsample. Therefore, Andreas was formerly unclassified. Andreas also showed tendencies of economic argumentations although the subjective theory did not emerge clearly. This means, economic arguments could only be identified on the level of concepts in the case of Andreas. This serves as further confirmation that the economical type was the most heterogeneous one out of the three types that were identified. Still, an economic perspective is important with respect to a complete understanding of resource dilemmas. For instance, the CBD (CBD, 1992) explicitly claims the protection of biodiversity through a sustainable utilisation. In that, pupils of the economic type displayed interesting trains of thought that could in teaching practice gradually be extended to new domains, such as the difficult economic situation of plant collectors.

\section{Critical discussion}

With respect to the economical type, it could be argued that this heterogeneous type should not be classified under one type. On the other hand, the typology should be seen as an element that provides us with an overview on the situation within a sample rather than providing quantitative information on the sample structure. Patterns leading to differences between the two subsamples can be presented more easily. This is especially interesting as the types in the case of our study represent an underlying subjective theory. Moreover, the tendency of the participants to either show or refuse empathy with the plant collectors in a resource dilemma showed clear differences between the types. Although empathy was not a criterion for an assignment to one of the three types, the tendency to either show such empathy or not, is consistent with the pupils' affiliation to the ecological or balanced type. 
Therefore, arguments reflecting empathy can be seen as a validation of the types that emerged from the data.

A second disadvantage of the typology is the fact that, if we regard the absolute number of arguments alone (as demonstrated by figure 7.2-7.4), some pupils could be considered as being part of more than one type. For instance, Simone (German) also demonstrated the basic criteria for the ecological type as she applied ecological arguments in all three stages of the interview. Here, a close analysis of original textual material is necessary to identify the underlying subjective theory the respective type is based on. This means that the main criterion for an assignment to a certain type should always be the quality of statements that constitute a coding category. Nevertheless, we believe that a typology can still be an excellent tool to structure a sample in qualitative analyses, when the methods are implemented with care.

\section{Chilean and German subsample}

Regarding the subjective theories and the typology identified in this study, two notable phenomena distinguish the two samples:

i. The balanced type mainly consisted of German pupils. The only Chilean pupil within this type, Carlos, did not suggest social solutions.

ii. The second dilemma example, incorporating the Latin American medical plant Boldo, was not identified as a resource dilemma by a group of five Chilean pupils (ecological type, subgroup I).

Reasons contributing to the above mentioned phenomena are diverse. One reason might be the notion that the subjective theory "loss of biodiversity due to social reasons" is apparently less prominent among the Chilean pupils. A source of this finding could be attributed to a stronger disciplinary orientation of the curriculum in Chile, which may focus less on training interdisciplinary thinking. Although this explanation seems logic, the result contradicts our initial expectation that Chilean pupils would be more open towards social explanations for biodiversity loss due to the proximity of the biodiversity hotspot to their context. Furthermore, difficulties surrounding the recognition of the local example by Chilean pupils emerged. One explanation for this could be that a large presence of Boldo on local markets might lead to the interpretation that natural resources are abundant. Therefore, for biodiversity education, it must not simply be assumed that a higher sensitivity towards biodiversity loss is simply authentic just because it occurs on learners' doorsteps. Nevertheless, the example of Boldo shows that learners construct knowledge directly from 
their individual everyday-life experiences, which mislead their interpretations when talking about local biodiversity as was demonstrated by the Chilean pupils.

\section{Future Perspectives}

Besides the question of how the identified types are distributed in a larger sample, a further research problem of interest is, why some pupils are able to consider an interdisciplinary perspective, while others are not. In this respect, social psychological theories, such as the Value-Belief-Norm Theory (Stern et al., 1999; Stern, 2000) provide interesting approaches that can be used to explain individuals' commitment to protect the environment. Therefore, a further study that applies the theory to the context of biodiversity with further samples of German and Chilean adolescents could clarify such crucial differences amongst perceptions. Furthermore, to investigate positive and negative predictors for a commitment to protect biodiversity, would contribute to the exploration of another, important learning prerequisite. Together with the herewith-reported research, biodiversity education could then build upon a empirical, qualitative and quantitative basis. 

8 Was fördert eine Bereitschaft von Oberstufenschüler(inne)n, die Biodiversität zu schützen? - Eine quantitative Studie in Anlehnung an die Value-Belief-Norm-Theorie ${ }^{1}$

\section{1 Zusammenfassung und Abstract}

In der vorliegenden Fragebogenstudie wurde der Einfluss von Werten, Überzeugungen und Persönlichen Normen auf eine Bereitschaft von Jugendlichen $(n=217)$ untersucht, die Biodiversität zu schützen. Als theoretischer Bezugrahmen diente die Value-Belief-Norm (VBN) Theorie. Insgesamt haben vor allem der Wert „Universalismus“, die Überzeugung einer „Verantwortungszuschreibung und wahrgenommene Möglichkeit zu Handeln“ und „Persönliche Normen“ einen Einfluss auf a) Aktivismus b) Handeln im privaten Raum und c) nicht-aktivistischem, öffentlichen Handeln gezeigt. Aus zusätzlich zu den VBNKonstrukten eingeführten Skalen erwies sich sozioökonomische Problemwahrnehmung als positiver und Problemverneinung als negativer Prädiktor für die erhobenen Bereitschaften, Biodiversität zu schützen. Bildungsmaßnahmen mit dem Ziel, Biodiversität zu schützen sollten den Ergebnissen zu Folge vor allem a) eine positive Wertorientierung gegenüber der Natur fördern, b) eine Erarbeitung realistischer Handlungsmöglichkeiten zum Schutz der Biodiversität ins Zentrum stellen und c) neben ökologischen Sachverhalten vor allem soziale und ökonomische Facetten des Biodiversitätsverlusts thematisieren.

What Influences High School Students' Commitment to Protect Biodiversity? - A Quantitative Study Based on the Value-Belief-Norm Theory

Within the presented study, the influence of values, beliefs, and personal norms on young peoples' ( $\mathrm{n}=217$ ) commitment to protect biodiversity was assessed. The Value-Belief-Norm (VBN) theory formed the theoretical background of the study. The central results showed that, particularly, the value "universalism", an "ascription of responsibility and perceived ability to reduce threat" and "personal norms" were strong predictors for three assessed behavioral commitments namely, activism, non-activist, public sphere behavior and privatesphere behavior. Out of four scales, which were included in addition to the VBN constructs, socio-economic problem perception proved to be positive and problem denial negative

1 Source: Menzel, S., \& Bögeholz, S. (2007). Was fördert eine Bereitschaft von Oberstufenschüler(inne)n, die Biodiversität zu schützen? - Eine quantitative Studie in Anlehnung an die Value-Belief-Norm-Theorie. Manuscript submitted for publication (Umweltpsychologie). 
predictor for all three behavioural commitments. According to our results, educational measures should a) foster a positive value orientation towards nature, b) offer realistic behavioral perspectives for protecting biodiversity and c) include social and economic facets of biodiversity loss rather than purely concentrating on ecological facts.

\subsection{Fragestellung}

Der Verlust der Biodiversität: Eine Herausforderung für Schule und Unterricht

Der Schutz der biologischen Vielfalt oder, synonym, Biodiversität zählt zu den zentralen Herausforderungen unseres Jahrhunderts (vgl. Convention on Biological Diversity (CBD), 1992). Das Phänomen des Biodiversitätsverlusts ist jedoch komplex, vor allem aus zwei Gründen. Erstens umfasst die Definition von Biodiversität drei Ebenen (CBD, Artikel 2):

1. Die Ebene der Artenvielfalt,

2. die Ebene der Vielfalt der Ökosysteme und

3. die Ebene der genetischen Vielfalt innerhalb der Arten.

Zweitens spielen für den Biodiversitätsverlust neben ökologischen vor allem ökonomische und soziale Faktoren eine Rolle. Die Gefährdung der Biodiversität tritt besonders an sogenannten Biodiversitäts-Hotspots auf, Gegenden, die häufig in sogenannten Entwicklungsländern liegen und in denen ein hoher Anteil der ursprünglich vorkommenden Arten bereits verloren ist (Mittermeier et al., 2004). Nicht selten führen an BiodiversitätsHotspots klassische Ressourcen-Nutzungsdilemmata, in denen arme Menschen ökonomisch von der Nutzung natürlicher Ressourcen abhängen, zum Rückgang der Biodiversität (Ernst, 1997; Hardin, 1968).

Die Convention on Biological Diversity (CBD, 1992) ist der wichtigste völkerrechtlich verbindliche Vertrag zum Schutz der Biodiversität. In Artikel 13a der CBD wird die zentrale Rolle von Bildungsmaßnahmen herausgestellt. Um gezielt Bildungsmaßnahmen konzipieren zu können, sind Erkenntnisse über psychologische Wirkmechanismen hilfreich, die positiven oder negativen Einfluss auf die Bereitschaft haben können, Biodiversität zu schützen.

\section{Stand der Forschung: Die Value-Belief-Norm Theorie}

Als theoretischer Bezugsrahmen wurde für unsere Studie die Value-Belief-Norm (VBN) Theorie (Stern et al., 1999; Stern, 2000) gewählt. Die VBN Theorie basiert auf dem Normaktivationsmodell (Schwartz \& Howard, 1981), das altruistisches Verhalten in Rückbezug auf moralische Werte und Normen erklärt. Obwohl das Normaktivationsmodell 
zur Erklärung altruistischen Verhaltens entwickelt wurde, ist es in der Vergangenheit erfolgreich eingesetzt worden, um umweltfreundliches Verhalten zu erklären (z.B. Guagnano et al., 1994; Hopper \& Nielsen, 1991; Stern et al., 1993; Widegren, 1998). Stern et al. (1993) erweiterten das Normaktivationsmodell um die Differenzierung der wahrgenommenen Konsequenzen eines Umweltproblems in egoistische und biosphärische Konsequenzen. Zusätzlich wurden altruistische, biosphärische und egoistische Werthaltungen als Prädiktoren für eine umweltbezogene Handlungsabsicht angenommen. Auf dieser Erweiterung basiert die VBN Theorie (Stern et al., 1999; Stern, 2000) (vgl. Abb. 1).

Als stabilster Einflussfaktor werden in der VBN Theorie altruistische, egoistische oder biosphärische Werte angenommen. Um eine grundsätzliche Werteorientierung als Prädiktor für Umwelthandeln zu testen, wurden erfolgreich die zehn universellen menschlichen Werte nach Schwartz (1992) eingesetzt. Die Schwartz-Werte können zu vier sogenannten WerteClustern zusammen gefasst werden: 1. Selbst-Transzendenz, 2. Bewahrung, 3. SelbstErhöhung und 4. Offenheit für Veränderung (s. Tab. 1). Die Cluster Bewahrung und SelbstErhöhung repräsentieren in der VBN Theorie egoistische Wertorientierungen, der Cluster Self-Direction altruistische und der Cluster Selbst-Transzendenz biosphärische Wertorientierungen (Stern et al., 1993). Sowohl biosphärische, wie auch altruistische und egoistische Wertorientierungen können theoretisch einen positiven Einfluss auf Umweltschutzbereitschaften nehmen (Stern et al. 1993; Stern et al., 1999; Stern, 2000). In der Literatur wurde jedoch vornehmlich ein positiver Einfluss biosphärisch-altruistischer Werte berichtet (Nordlund \& Garvill, 2002; Schultz \& Zelezny, 1999; Stern et al., 1993).

Tab. 8.1. Die universellen menschlichen Werte nach Schwartz (1992), eigene Übersetzung, Hedonismus vereint Elemente zweier Cluster.

\begin{tabular}{|c|c|}
\hline $\begin{array}{l}\text { Werte-Cluster } \\
\text { (englische Bezeichnung) }\end{array}$ & Einzelwerte (englische Bezeichnung) \\
\hline $\begin{array}{l}\text { Selbst-Transzendenz } \\
\text { (Self-Transcendence) }\end{array}$ & $\begin{array}{ll}\text { - } & \text { Universalismus (Universalism) } \\
\text { - } & \text { Benevolenz, Mildtätigkeit (Benevolence) }\end{array}$ \\
\hline $\begin{array}{l}\text { Bewahrung } \\
\text { (Conservation) }\end{array}$ & $\begin{array}{ll}\text { - } & \text { Gleichförmigkeit (Conformity) } \\
\text { - } & \text { Tradition (Tradition) } \\
\text { - } & \text { Sicherheit (Security) }\end{array}$ \\
\hline $\begin{array}{l}\text { Selbst-Erhöhung } \\
\text { (Self-Enhancement) }\end{array}$ & $\begin{array}{ll}- & \text { Macht (Power) } \\
\text { - } & \text { Ehrgeiz (Achievement) } \\
\end{array}$ \\
\hline $\begin{array}{l}\text { Offenheit für Veränderung } \\
\text { (Openness to Change) }\end{array}$ & $\begin{array}{ll}\text { - } & \text { Stimulation (Stimulation) } \\
\text { - } & \text { Selbstbestimmung (Self-Direction) }\end{array}$ \\
\hline
\end{tabular}


Überzeugungen (,Beliefs“), werden durch drei übergeordnete Konstrukte repräsentiert:

1. Das New Environmental Paradigm (NEP, Dunlap \& van Liere, 1978; Dunlap et al., 2000), das der Erfassung einer allgemeinen positiven Einstellung zur Natur dient. Nach Stern et al. (1995) kann das NEP als „Folk Ecology“ zu betrachtet werden, nach der Menschen sich als von der Natur abhängig wahrnehmen. Ein starker direkter Zusammenhang zwischen dem NEP und Handlungsbereitschaften wurde empirisch bisher nicht nachgewiesen, da das NEP offensichtlich eher weitere umweltfreundliche Überzeugungen beeinflusst (z.B. Dunlap et al., 2000; Stern et al., 1995; Stern et al., 1999).

2. Egoistisches, altruistisches oder biosphärisches Bewusstsein für die Folgen eines Umweltproblems. Mit diesen Konstrukten wurde der Mediator des Normaktivationsmodells „Bewusstsein für die Konsequenzen einer altruistischen Handlung“ durch biosphärisches und, angelehnt an ökonomische Ansätze der egoistischen Bedürfnisbefriedigung (z.B. Hardin, 1968), egoistisches Bewusstsein für Konsequenzen eines Umweltproblems ergänzt (Stern et al., 1993; Stern et al., 1999). Schultz (2001) gelang grundsätzlich der empirische Nachweis der dreigeteilten Struktur von wahrgenommenen altruistischen, biosphärischen und egoistischen Konsequenzen eines Umweltproblems. Die Wahrnehmung von altruistischen und biosphärischen Konsequenzen wurde jedoch durch die gleichen WerteCluster nach Schwartz (1992) beeinflusst, was auf einen inhaltlichen Zusammenhang der Konstrukte hinweist.

3. Eine Verantwortungszuschreibung und wahrgenommene Möglichkeit zu Handeln (Ascription of Responsibilty und Perceived Ability to Reduce Threat). Das Konstrukt wirkt nach dem Normaktivationsmodell ebenfalls als Mediator auf die Ausbildung einer Persönlichen Norm. Ein direkter Einfluss des Konstrukts auf eine Handlungsbereitschaft im Umweltbereich konnte von Guagnano und Kollegen nachgewiesen werden (z.B. Guagnano et al., 1994). Übereinstimmend identifizierten Kaiser, Ranney, Hartig und Bowler (1999) Verantwortungsüberzeugung als eine von drei zentralen unabhängigen Variablen für eine allgemeine ökologische Handlungsbereitschaft.

Persönliche Normen („Personal Norms“) sind der dritte Baustein der VBN-Theorie. Persönliche Normen können, dem Normaktivationsmodell entsprechend, wiederum von vorgeordneten Konstrukten im Modell beeinflusst werden (s. Abb. 1). Eine hohe Persönliche Norm in Bezug auf Umwelthandeln wird theoretisch als stärkster Prädiktor für selbst erklärtes Umwelthandeln angenommen. Bamberg und Möser (2007) berichteten als ein Resultat von Meta-Analysen zur Erklärung umweltfreundlichen Verhaltens einen Anstieg erklärter Varianz zwischen 1-10\%, wenn moralische Normen als zusätzlicher unabhängiger Prädiktor angenommen wurden. 
Als abhängige Variable wird im Rahmen der VBN-Theorie zwischen vier Handlungsbereitschaften unterschieden (vgl. Stern, 2000): 1. Aktivismus („activism“) wie beispielsweise das Entrollen eines Protesttransparents vor der Fassade einer Firma, die umweltschädigende Produktionsverfahren anwendet, 2. Nicht-aktivistisches, öffentliches Handeln („non activist, public-sphere behavior") wie das Unterzeichnen einer Unterschriftenliste zur Forderung von verbessertem Umweltschutz, 3. Handeln im privaten Raum („private-sphere behavior") wie beispielsweise Mülltrennung in privaten Haushalten und 4. Handeln in Organisationen (,,behavior in organisations“) wie der Einsatz für die Umwelt im Rahmen der Institution, für die eine Person beruflich oder durch freiwilliges Engagement tätig ist.

Verschiedene Studien erklärten mit von eineander unabhängigen Prädiktoren aus den Bereichen der Werte, Überzeugungen und Normen umweltfreundliches Verhalten (Bamberg \& Möser, 2007; Guagnano et al., 1994; Kaiser et al., 1999; Kaiser \& Shimoda, 1999). Analog zu den genannten Studien diente die VBN-Theorie in der hier präsentierten Forschungsarbeit als Rahmen. Eine Überprüfung der Kausalstruktur sowie eine Differenzierung in Prädiktoren und Mediatoren erfolgten im Rahmen der hier präsentierten Ergebnisse nicht.

Die Wahrnehmung sozioökonomischer und ökologischer Auslöser für den Verlust der Biodiversität

Zusätzlich zu den Konstrukten der VBN Theorie nahmen die Autorinnen weitere Einflussfaktoren auf die Bereitschaft von Jugendlichen an, die Biodiversität zu schützen. Für Bildungsmaßnahmen kann es aus konstruktivistischer Sicht hilfreich sein, Lernvoraussetzungen wie beispielsweise individuelle Vorstellungen von Schüler(inne)n zu kennen. Im Rahmen unseres Projekts wurde daher zunächst eine explorative Phase durchgeführt, in der 16 bis 17-jährige Schüler(innen) $(n=12)$ interviewt wurden (Menzel \& Bögeholz, 2006). Nach einer deduktiven Analyse konnten subjektive Theorien zum Verlust der Biodiversität identifiziert werden. Subjektive Theorien sind individuelle Sinnsysteme, die handlungssteuernd sein können und eine ähnliche Erklärungsfunktion wie wissenschaftliche Theorien haben (Groeben et al., 1988). Sie dienen somit der Realitätskonstituierung, vor allem bei einer Konfrontation mit Neuem. Subjektive Theorien sind auf Grund ihres realitätskonstituierenden Charakters eine didaktische Herausforderung, wenn sie einem neu zu erlernenden Inhalt entgegen stehen (Vosniadou, 1992). Eine im Rahmen unserer Studie identifizierte subjektive Theorie ist in dieser Hinsicht hervorzuheben: „Biodiversitätsverlust durch ökologische Ursachen“. Sie führte bei den 
befragten Schüler(inne)n dazu a) ökologische Zusammenhänge isoliert von ökonomischen und sozialen Faktoren zu betrachten, b) geringe Empathie mit Menschen in ökologischsozialen Dilemmata zu zeigen und das Problem des Biodiversitätsverlusts zu verneinen und c) eine nicht immer ökologisch fachlich fundierte Betrachtungsweise zu zeigen. Beispielsweise wurden falsche ökologische Zusammenhänge angeführt, wenn eine zufriedenstellende Erklärung des Biodiversitätsverlusts nicht auf Grund von ökologischer Sachkompetenz erreicht werden konnte. Eine weitere subjektive Theorie, „Biodiversitätsverlust durch soziale Ursachen“ ließ hingegen eine Betrachtung von ökologischen, ökonomischen und sozialen Aspekten gleichermaßen zu, was in der Regel zu einer interdisziplinären - und somit erfolgreichen - Rekonstruktion der Problematik führte.

\section{Hypothesen und Forschungsfragen}

Aus den Ergebnissen der qualitativen Phase wurde als erste Forschungsfrage aufgeworfen, inwiefern zusätzliche, aus der qualitativen Phase abgeleitete Konstrukte zu sozioökonomischer und ökologischer Problemwahrnehmung sowie Wissen und Problemverneinung einen Einfluss auf eine Bereitschaft von Schüler(inne)n haben, die Biodiversität zu schützen. Zudem gingen wir folgenden Hypothesen nach:

1. Die Werte des Clusters Selbst-Transzendenz (Universalismus und Benevolenz) sind relevante Prädiktoren für Bereitschaften von Jugendlichen, Biodiversität zu schützen.

2. Je stärker Oberstufenschüler(innen) von ihrer Verantwortung für den Schutz der Biodiversität überzeugt sind, desto höhere Bereitschaften zeigen sie, die Biodiversität zu schützen.

3. Persönliche Normen sind ein starker Einflussfaktor auf Bereitschaften von Jugendlichen, Biodiversität zu schützen.

Neben der Prüfung dieser Hypothesen war eine zweite Forschungsfrage, welche Prädiktoren sich neben Universalismus, Benevolenz, eigener Verantwortungszuschreibung und Persönlichen Normen als relevant für die drei untersuchten Handlungsbereitschaften erweisen. 


\section{3 Methodik}

\section{Stichprobe und Skalen}

Die Stichprobe ( $\mathrm{n}=217)$ umfasste 125 Schülerinnen und 92 Schüler der elften Jahrgangsstufe im Alter von 16 bis 19 Jahren. Die Fragebögen wurden im Januar 2006 in Klassensätzen eingesetzt. Um eine möglichst große Heterogenität innerhalb der Stichprobe zu erzielen, wurden Gymnasien und Gesamtschulen aus verschiedenen Umgebungskontexten (zum Teil aus großstädtischem Raum, zum Teil aus Kleinstädten oder vom Land) in die Studie einbezogen. Eine Repräsentativität der Stichprobe wurde nicht angestrebt. Alle Konstrukte (abgesehen von denen im Original eingesetzten Skalen zur Erfassung der Schwartz-Werte und des NEP) wurden auf den Kontext der Biodiversität und eine Stichprobe mit Oberstufenschüler(inne)n angepasst. Basierend auf dem in Abb. 1 dargestellten Modell wurde ein Fragebogen entwickelt.

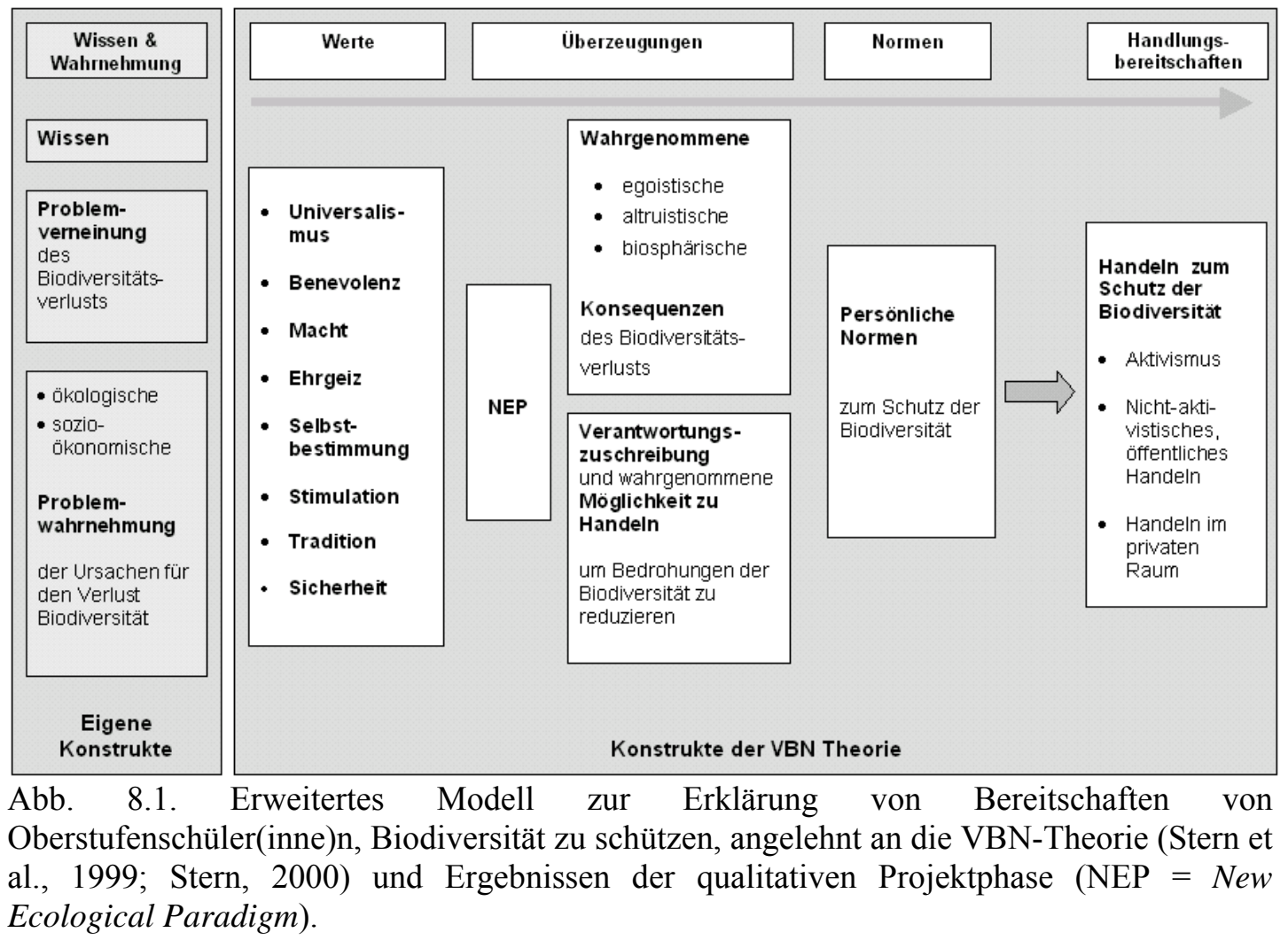

Als abhängige Variable dienten in unserer Studie drei der vier in der VBN Theorie postulierten Handlungsbereitschaften, nämlich a) Aktivismus, b) nicht-aktivistisches, öffentliches Handeln und c) Handeln im privaten Raum. Zur Erfassung der Werte wurde der von Schwartz (2005) entwickelte Portrait Value Questionnaire eingesetzt. Proband(inn)en 
wurden hier Aussagen zu hypothetischen Personen vorgelegt, die jeweils einen Aspekt des zu testenden menschlichen Wertes verdeutlichen. Der Wert Hedonismus steht theoretisch und empirisch zwischen zwei Clustern, Gleichförmigkeit hingegen hängt eng mit dem Wert Tradition zusammen (Schwartz, 1992). Als Konsequenz wurden die beiden Werte aus unseren Analysen ausgeschlossen (vgl. Tab. 1). Das NEP wurde mit der Revised NEP Skala erhoben (Dunlap et al., 2000). Die eigene Übersetzung der Skala aus dem Englischen wurde mittels Rückübersetzung abgesichert. Die Skalen für wahrgenommene egoistische, biosphärische und altruistische Konsequenzen wurden in enge Anlehnung an Stern et al. (1999) konstruiert, jedoch auf den Kontext des Biodiversitätsverlusts angepasst. Ebenso wurde mit der Skala für Verantwortungszuschreibung und wahrgenommene Möglichkeit zu Handeln verfahren. Persönliche Normen wurden in Anlehnung an Stern et al. (1999) und Widegren (1998) mit Items zu einem guten Gewissen bei Handlungen zur Bewahrung der Biodiversität und entsprechend schlechtem Gewissen bei Handlungen, die eine Schädigung der Biodiversität mit sich bringen, operationalisiert.

Neben den VBN-Konstrukten wurde ausgehend von den Ergebnissen der qualitativen Projektphase ein weiterer Block Wissen und Problemwahrnehmung eingefügt. Als zusätzliche Konstrukte kamen dabei a) biodiversitätsbezogenes Wissen, b) ökologische und c) sozioökonomische Problemwahrnehmung und d) Problemverneinung zum Tragen. Die Konstrukte für Wissen und Problemwahrnehmung wurden zum Teil basierend auf Schüleraussagen aus der Interviewstudie konstruiert. Der biodiversitätsbezogene Wissenstest deckte dabei nicht nur ökologische, sondern zu gleichen Teilen soziale und ökonomische Aspekte ab. Beispielitems der nicht im Original übernommenen Skalen sind in Tab. 2 aufgeführt.

Tab. 8.2. Konstrukte und Beispielitems der Studie, die nicht im Original übernommen wurden.

\begin{tabular}{|l|l|}
\hline Konstrukt & \multicolumn{1}{|c|}{ Beispiel } \\
\hline $\begin{array}{l}\text { Wahrgenommene egoistische } \\
\text { Konsequenzen }\end{array}$ & $\begin{array}{l}\text { Der Verlust der biologischen Vielfalt wird für mich und } \\
\text { meine Familie ernsthafte Konsequenzen haben. }\end{array}$ \\
\hline $\begin{array}{l}\text { Wahrgenommene altruistische } \\
\text { Konsequenzen }\end{array}$ & $\begin{array}{l}\text { Leidtragende unter dem Verlust der biologischen Vielfalt } \\
\text { sind vor allem auch die Menschen künftiger Generationen. }\end{array}$ \\
\hline $\begin{array}{l}\text { Wahrgenommene } \\
\text { biosphärische Konsequenzen }\end{array}$ & $\begin{array}{l}\text { Innerhalb der nächsten Jahrzehnte werden Tausende Arten } \\
\text { aussterben, was vor allem schlimme Konsequenzen für die } \\
\text { Natur hat. }\end{array}$ \\
\hline \multirow{2}{*}{$\begin{array}{l}\text { Verantwortungszuschreibung } \\
\text { und wahrgenommene } \\
\text { Möglichkeit zu Handeln }\end{array}$} & $\begin{array}{l}\text { Jede/r einzelne ist verpflichtet, sein oder ihr Möglichstes } \\
\text { zum Schutz der biologischen Vielfalt auf der Welt zu tun. }\end{array}$ \\
\cline { 2 - 2 } & $\begin{array}{l}\text { Jeder Mensch, auch ich, kann dazu beitragen, die Arten in } \\
\text { unserer Region zu schützen. }\end{array}$ \\
\hline
\end{tabular}


Tabelle 8.2 (Fortsetzung)

\begin{tabular}{|c|c|}
\hline \multirow{2}{*}{ Persönliche Normen } & $\begin{array}{l}\text { Ich habe ein schlechtes Gewissen, wenn ich den Schutz der } \\
\text { biologischen Vielfalt in der Welt nicht unterstütze. }\end{array}$ \\
\hline & $\begin{array}{l}\text { Mein Gewissen sagt mir, dass ich mehr tun sollte, um } \\
\text { Arten in unserer Region zu schützen. }\end{array}$ \\
\hline Aktivismus & $\begin{array}{l}\text { Ich würde Protest-Transparente vor der Zentrale einer } \\
\text { Firma anbringen, von der ich weiß, dass sie zum } \\
\text { Artensterben beiträgt. }\end{array}$ \\
\hline $\begin{array}{l}\text { Nichtaktivistisches, } \\
\text { öffentliches Handeln }\end{array}$ & $\begin{array}{l}\text { Ich würde einen Brief an die Regierung schreiben, um auf } \\
\text { den notwendigen Schutz der biologischen Vielfalt } \\
\text { hinzuweisen. }\end{array}$ \\
\hline Handeln im privaten Raum & $\begin{array}{l}\text { Ich würde selbst Arten schützen (z.B. beim Wandern die } \\
\text { Wege nicht verlassen, um die Arten dort nicht zu stören). }\end{array}$ \\
\hline $\begin{array}{l}\text { Problemwahrnehmung } \\
\text { ökologisch }\end{array}$ & $\begin{array}{l}\text { Biologische Vielfalt ist hauptsächlich gefährdet, weil es } \\
\text { nicht genügend Lebensräume auf der Welt gibt, in denen } \\
\text { sich Tiere und Pflanzen ungestört vermehren können. }\end{array}$ \\
\hline $\begin{array}{l}\text { Problemwahrnehmung } \\
\text { sozioökonomisch }\end{array}$ & $\begin{array}{l}\text { Biologische Vielfalt ist hauptsächlich gefährdet, weil } \\
\text { Gesetze zum Schutz der biologischen Vielfalt vor } \\
\text { übermäßigem Handel in unserer Region nicht ausreichen. }\end{array}$ \\
\hline Problemverneinung & $\begin{array}{l}\text { Viele Berichte über den Rückgang der Biodiversität auf } \\
\text { der Welt sind übertrieben. }\end{array}$ \\
\hline
\end{tabular}

\section{Analysen}

Kolinearitäten zwischen Prädiktorvariablen konnten durch VIF-Werte ausgeschlossen werden. Unabhängige Variablen korrelierten in der Regel nicht höher als $r=.55$. Ausnahmen bildeten die Schwartz-Werte Universalismus und Benevolenz (Spearman's Rho =.60). Einige Konstrukte wiesen eine Verletzung der Normalverteilungsannahme auf, was durch ein signifikantes Ergebnis des Kolmogorov-Smirnov Anpassungstests ermittelt wurde (vgl. Tab. 3). Alle nicht normalverteilten Konstrukte wiesen allerdings eine gleichgerichtete Verteilung auf, was die Durchführung von linearen Regressionen ermöglichte (Field, 2005). Regressionen gelten zudem als robust gegenüber der Normalverteilungsverletzung, wenn die Fehler der Variablen gleich verteilt sind (Field, 2005). Durch fallweise Diagnose standardisierter Residuen größer als 2 und Cook's Distance (Field, 2005) konnten keine Ausreißer identifiziert werden, die unsere Regressionsmodelle übermäßig beeinflussten. Die Homogenität der Varianzen (Homoskedastizität) war in Regressionen mit Problemverneinung als Prädiktor eingeschränkt. Ähnliches konnte für ökologische und sozioökonomische Problemwahrnehmung und biodiversitätsbezogenes Wissen in der multiplen Regression zur Erklärung der Bereitschaft zu Aktivismus festgestellt werden. 
Tab. 8.3. Deskriptive Kennwerte der im Fragebogen eingesetzten Skalen.

\begin{tabular}{|c|c|c|c|c|c|c|}
\hline Bereich & Skala & Items & Mean & SD & $\begin{array}{c}\text { K-S } \\
\text { Test } \\
P\end{array}$ & $\begin{array}{c}\text { Cron- } \\
\text { bach's } \\
\quad \alpha\end{array}$ \\
\hline \multirow{8}{*}{ Werte* } & Selbstbestimmung & 4 & 3.52 & 0.82 & .02 & .57 \\
\hline & Macht & 3 & 1.97 & 1.11 & .01 & .42 \\
\hline & Universalismus & 6 & 3.12 & 0.95 & .03 & .78 \\
\hline & Ehrgeiz & 4 & 2.95 & 1.10 & .23 & .80 \\
\hline & Sicherheit & 5 & 2.88 & 0.97 & .30 & .68 \\
\hline & Stimulation & 3 & 3.09 & 1.03 & .03 & .64 \\
\hline & Tradition & 4 & 2.14 & 0.92 & .01 & .48 \\
\hline & Benevolenz & 4 & 3.47 & 0.85 & .00 & .63 \\
\hline \multirow{5}{*}{$\begin{array}{l}\text { Überzeu- } \\
\text { gungen }\end{array}$} & $\begin{array}{l}\text { Wahrgenommene egoistische } \\
\text { Konsequenzen }\end{array}$ & 3 & 0.98 & 0.46 & .01 & .40 \\
\hline & $\begin{array}{l}\text { Wahrgenommene altruistische } \\
\text { Konsequenzen }\end{array}$ & 3 & 1.98 & 0.54 & .04 & .61 \\
\hline & $\begin{array}{l}\text { Wahrgenommene biosphärische } \\
\text { Konsequenzen }\end{array}$ & 3 & 2.32 & 0.48 & .00 & .56 \\
\hline & $\begin{array}{l}\text { Verantwortungszuschreibung und } \\
\text { wahrgenommene Möglichkeit zu Handeln }\end{array}$ & 16 & 1.72 & 0.61 & .73 & .89 \\
\hline & New Ecological Paradigm & 15 & 2.11 & 0.38 & .03 & .71 \\
\hline Normen & Persönliche Normen & 8 & 1.10 & 0.75 & .12 & .90 \\
\hline Wissen $* *$ & Biodiversitätsbezogenes Wissen & 25 & 0.67 & 0.15 & .00 & .61 \\
\hline \multirow{3}{*}{$\begin{array}{c}\text { Wahr- } \\
\text { nehmung }\end{array}$} & Problemwahrnehmung ökologisch & 8 & 1.91 & 0.54 & .16 & .68 \\
\hline & Problemwahrnehmung sozioökonomisch & 16 & 1.79 & 0.53 & .41 & .79 \\
\hline & Problemverneinung & 8 & 0.66 & 0.54 & .01 & .80 \\
\hline \multirow{3}{*}{ Handeln } & Aktivismus & 4 & 0.79 & 0.80 & .00 & .83 \\
\hline & Nichtaktivistisches, öffentliches Handeln & 4 & 1.45 & 0.77 & .02 & .65 \\
\hline & Handeln im privaten Raum & 4 & 1.44 & 0.84 & .18 & .76 \\
\hline \multicolumn{7}{|c|}{$\begin{array}{l}\text { Alle nicht gekennzeichneten Skalen vierstufig: Stimme zu (3), stimme weitgehend zu (2), stimme } \\
\text { teilweise zu (1), stimme nicht zu (0) } \\
\text { * sechsstufige Antwortskala: Sehr wie ich (5), wie ich (4), etwa wie ich (3), ein wenig wie ich (2), } \\
\text { nicht wie ich (1), überhaupt nicht wie ich (0) } \\
\text { ** dichotome Antwortskala: Stimme zu (1), stimme nicht zu (0) }\end{array}$} \\
\hline
\end{tabular}

Cronbach's Alpha lag für alle Skalen zwischen $\alpha=.60$ und $\alpha=.90$ abgesehen von den Skalen für Selbstbestimmung $(\alpha=0,57)$, Macht $(\alpha=0,42)$ und Tradition $(\alpha=0,48)$ sowie den Skalen für wahrgenommene egoistische $(\alpha=0,40)$ und biosphärische $(\alpha=0,56)$ Konsequenzen des 
Biodiversitätsverlusts. Kennwerte und deskriptive Ergebnisse der Skalen sind in Tab. 3 dargestellt.

Um den Einfluss der Konstrukte auf die drei Handlungsbereitschaften zu ermitteln, wurden lineare einfache und multiple Regressionen durchgeführt. Für alle Analysen wurde das Einschlussverfahren gewählt. Die Klassifikation der Effektstärken wurde in Anlehnung an Bortz (1993, S. 429) vorgenommen $\left(\mathrm{R}^{2}>.0196=\right.$ schwacher Effekt; $\mathrm{R}^{2}>.1304=$ mittlerer Effekt; $\mathrm{R}^{2}>.2593=$ starker Effekt). Die Regressionsmodelle wurden in zwei analytischen Schritten erstellt.

In einem ersten Verfahren (Analyse I) wurde die Prädiktorkraft einzeln für jeden der theoretischen Blöcke Werte, Überzeugungen, Persönliche Normen sowie Wissen und Problemwahrnehmung bestimmt (vgl. Abb.1). Diese blockweise getrennten Regressionen wurden für jede der drei untersuchten Handlungsbereitschaften durchgeführt.

Konstrukte, die sich in Analyse I als wirkmächtige Prädiktoren erwiesen haben, flossen in Analyse II für die jeweilige Handlungsbereitschaft in ein Gesamtmodell ein, das sich über alle Konstrukte des erweiterten Modells erstreckte (vgl. Abb. 1). Folglich wurde in Analyse II für die drei Handlungstypen je ein Gesamtmodell erstellt.

\subsection{Ergebnisse}

Analyse I

In Analyse I hat sich im Bereich der Werte gezeigt, dass Universalismus für alle Handlungsbereitschaften der wirkmächtigste positive Prädiktor war (vgl. Tab. 4). Benevolenz (als zweiter Wert des Clusters Selbst-Transzendenz) zeigte hingegen keinen Einfluss. Neben den Werten des Clusters Selbst-Transzendenz war Selbstbestimmung positiver Prädiktor für Handeln im privaten Raum. Ebenfalls negativ wirkte sich Stimulation auf eine Handlungsbereitschaft im privaten Raum aus. Sicherheit erwies sich als ähnlich starker, aber negativer Prädiktor für eine Handlungsbereitschaft zu Aktivismus. Im Bereich der Überzeugungen hat sich das Konstrukt der Verantwortungszuschreibung und wahrgenommenen Möglichkeit zu Handeln für alle Handlungsbereitschaften als positiver Prädiktor erwiesen. Zusätzlich war aus dem Bereich der Überzeugungen das NEP relevant für alle erhobenen Handlungsbereitschaften. Daneben erwies sich die Wahrnehmung biosphärischer Konsequenzen wirkmächtig für eine Bereitschaft zum Handeln im privaten Raum. Das Konstrukt Persönliche Normen war wichtiger Prädiktor für alle Handlungstypen. Zudem verfügten Persönliche Normen in Analyse I für alle Handlungstypen über das höchste $\beta$-Gewicht $(\beta=.53-.61)$. Aus dem Block Wissen und 
Problemwahrnehmung war sozioökonomische Problemwahrnehmung für alle gemessenen Handlungsbereitschaften positiver, Problemverneinung negativer Prädiktor. Im Vergleich aller Modelle aus Analyse I lieferte das Regressionsmodell des Blocks Wissen und Problemwahrnehmung die niedrigste Varianzaufklärung $\left(\mathrm{R}^{2}=.10-.21\right)$. Die Modelle der VBN Konstrukte (Werte, Überzeugungen und Persönliche Normen) wiesen in Analyse I für alle drei Handlungstypen starke oder mittlere Effekte auf. Wissen und Problemwahrnehmung zeigten mittlere oder schwache Effekte zur Erklärung der drei gemessenen Handlungstypen.

Tab. 8.4. Ergebnisse der Analyse I: Regressionen für die Bereitschaft zu drei verschiedenen Handlungstypen.

\begin{tabular}{|c|c|c|c|c|}
\hline Blöcke & $\begin{array}{l}\text { Kriterium } \rightarrow \\
\text { Prädiktoren } \\
\downarrow\end{array}$ & Aktivismus & $\begin{array}{l}\text { Nicht-akti- } \\
\text { vistisches, } \\
\text { offentliches } \\
\text { Handeln }\end{array}$ & $\begin{array}{l}\text { Handeln im } \\
\text { privaten Raum }\end{array}$ \\
\hline & & \multicolumn{3}{|c|}{ standardisierte $\beta$-Koeffizienten } \\
\hline \multirow{11}{*}{$\sum_{3}^{0}$} & Selbstbestimmung & n.s. & n.s. & $.24 * *$ \\
\hline & Macht & n.s. & n.s. & n.s. \\
\hline & Universalismus & $.54 * * *$ & $.51 * * *$ & $.54 * * *$ \\
\hline & Ehrgeiz & n.s. & n.s. & n.s. \\
\hline & Sicherheit & $-.16^{*}$ & n.s. & n.s. \\
\hline & Stimulation & n.s. & n.s. & $-.25^{* * *}$ \\
\hline & Tradition & n.s. & n.s. & n.s. \\
\hline & Benevolenz & n.s. & n.s. & n.s. \\
\hline & $R^{2}$ & $.29+++$ & $.31+++$ & $.38+++$ \\
\hline & $R^{2}$ (korr.) & .26 & .29 & .36 \\
\hline & F-Wert & $10.38 * * *$ & $11.80^{* * *}$ & $16.00 * * *$ \\
\hline \multirow{8}{*}{ 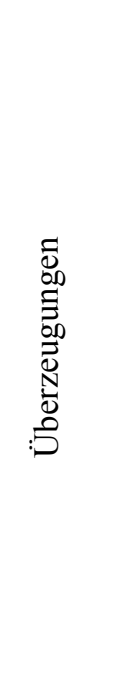 } & $\begin{array}{l}\text { Wahrgenommene egoistische } \\
\text { Konsequenzen }\end{array}$ & n.s. & n.s. & n.s. \\
\hline & $\begin{array}{l}\text { Wahrgenommene altruistische } \\
\text { Konsequenzen }\end{array}$ & n.s. & n.s. & n.s. \\
\hline & $\begin{array}{l}\text { Wahrgenommene biosphärische } \\
\text { Konsequenzen }\end{array}$ & n.s. & n.s. & $.15^{*}$ \\
\hline & $\begin{array}{l}\text { Verantwortungszuschreibung und } \\
\text { wahrgenommene Möglichkeit zu } \\
\text { Handeln }\end{array}$ & $.37 * * *$ & $.36 * * *$ & $.44 * * *$ \\
\hline & New Ecological Paradigm & $.18^{*}$ & $.21 * *$ & $.21^{* *}$ \\
\hline & $R^{2}$ & $.24++$ & $.29+++$ & $.36+++$ \\
\hline & $R^{2}$ (korr.) & .22 & .27 & .35 \\
\hline & F-Wert & $13.15^{* * *}$ & $17.07 * * *$ & $23.76^{* * *}$ \\
\hline
\end{tabular}


Tabelle 8.4 (Fortsetzung)

\begin{tabular}{|c|c|c|c|c|}
\hline \multirow{4}{*}{ 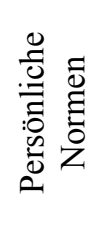 } & Persönliche Normen & $.61 * * *$ & $.53 * * *$ & $.57 * * *$ \\
\hline & $R^{2}$ & $.38+++$ & $.28+++$ & $.33+++$ \\
\hline & $R^{2}$ (korr.) & .37 & .28 & .32 \\
\hline & F-Wert & $127.85^{* * *}$ & $85.82 * * *$ & $104.10^{* * *}$ \\
\hline \multirow{7}{*}{ 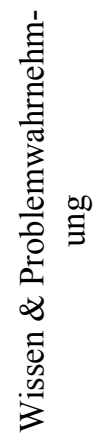 } & Biodiversitätsbezogenes Wissen & n.s. & n.s. & n.s. \\
\hline & Problemwahrnehmung ökologisch & n.s. & n.s. & n.s. \\
\hline & $\begin{array}{l}\text { Problemwahrnehmung } \\
\text { sozioökonomisch }\end{array}$ & $.15^{*}$ & $.16^{*}$ & $.18^{*}$ \\
\hline & Problemverneinung & $-.15^{*}$ & $-.34 * * *$ & $-.32 * * *$ \\
\hline & $R^{2}$ & $.10+$ & $.21++$ & $.21++$ \\
\hline & $R^{2}$ (korr.) & .08 & .20 & .19 \\
\hline & F-Wert & $5.50^{* * *}$ & $14.09^{* * *}$ & $13.61 * * *$ \\
\hline
\end{tabular}

\section{Analyse II}

Die für Analyse II entwickelten Gesamtmodelle sind in Tab. 5 aufgeführt. Für Aktivismus waren Persönliche Normen mit dem höchsten standardisierten $\beta$-Gewicht $(\beta=.41 ; p<.001)$ stärkster positiver Prädiktor. Universalismus zeigte den zweitstärksten positiven Einfluss $(\beta=.30 ; p<.001)$, Sicherheit einen leicht negativen Einfluss $(\beta=.14 ; p<.05)$. Die Varianzaufklärung der Prädiktoren des Gesamtmodells für eine Bereitschaft zu Aktivismus betrug $47 \%$.

Für eine Bereitschaft zu nicht-aktivistischem, öffentlichen Handeln (Tab. 5) war Universalismus wichtigster Prädiktor $(\beta=.29 ; \quad \mathrm{p}<.001)$. Persönliche Normen waren zweitstärkster positiver Prädiktor $(\beta=.25 ; \mathrm{p}<.001)$. Verantwortungszuschreibung und wahrgenommene Möglichkeit zu Handeln zeigte zudem einen leicht positiven Einfluss $(\beta=.14 ; p<.05)$, Problemverneinung einen leicht negativen Einfluss $(\beta=-.15 ; p<.05)$. Die Prädiktoren des Gesamtmodells erklärten in unserer Stichprobe $46 \%$ der Varianz dieser Handlungsbereitschaft.

Auch für Handeln im privaten Raum hat sich Universalismus als stärkster Einflussfaktor erwiesen $(\beta<0.27 ; p<.001)$. Weiterhin waren Persönliche Normen zweitstärkster Prädiktor $(\beta=.26 ; p<.001)$. Der Wert Stimulation zeigte zusätzlich einen negativen $(\beta=-.22 ; p<.001)$, der Wert Selbstbestimmung einen leicht positiven Einfluss $(\beta=.16 ; p<.01)$. Verantwortungszuschreibung und wahrgenommene Möglichkeit zu Handeln hatte wiederum einen positiven Einfluss $(\beta=.19 ; \mathrm{p}<.01)$. Das Gesamtmodell erklärte $55 \%$ der Varianz für 
Handeln im privaten Raum. Für alle Handlungstypen zeigten die Regressionsmodelle aus Analyse II eine hohe Varianzaufklärung.

Tab. 8.5. Gesamtmodell für die Bereitschaft, Biodiversität durch Aktivismus, nichtaktivistisches, öffentliches Handeln und Handeln im privaten Raum zu schützen (Analyse II). NEP = New Ecological Paradigm.

\begin{tabular}{|c|c|c|c|}
\hline Prädiktoren & Aktivismus & $\begin{array}{l}\text { Nicht- } \\
\text { aktivistisches, } \\
\text { offentliches } \\
\text { Handeln }\end{array}$ & $\begin{array}{l}\text { Handeln im } \\
\text { privaten } \\
\text { Raum }\end{array}$ \\
\hline Universalismus & $.30 * * *$ & $.29 * * *$ & $.27 * * *$ \\
\hline Sicherheit & $-.13 *$ & -- & -- \\
\hline Selbstbestimmung & -- & -- & $.16^{* *}$ \\
\hline Stimulation & -- & -- & $-.22 * * *$ \\
\hline NEP & n.s. & n.s. & n.s. \\
\hline $\begin{array}{l}\text { Wahrgenommene biosphärische } \\
\text { Konsequenzen }\end{array}$ & -- & -- & n.s. \\
\hline $\begin{array}{l}\text { Verantwortungszuschreibung und } \\
\text { wahrgenommene Möglichkeit zu Handeln }\end{array}$ & n.s. & $.14^{*}$ & $.19 * *$ \\
\hline Persönliche Normen & $.41 * * *$ & $.25 * * *$ & $.26^{* * *}$ \\
\hline Problemwahrnehmung sozioökonomisch & n.s. & n.s. & n.s. \\
\hline Problemverneinung & n.s. & $-.15^{*}$ & n.s. \\
\hline$R^{2}$ & $.47+++$ & $.46+++$ & $.55+++$ \\
\hline$R^{2}($ korr $)$ & .45 & .44 & .53 \\
\hline F-Wert & $25.75 * * *$ & $29.63 * * *$ & $27.69 * * *$ \\
\hline \multicolumn{4}{|c|}{$\begin{array}{l}* \mathrm{p}<.05 * * \mathrm{p}<.01 * * * \mathrm{p}<.001 \\
\text { Effekte: }+ \text { schwach }++ \text { mittel }+++ \text { stark (nach Bortz 1993. S. 429) } \\
--=\text { Variable n.s. in Analyse I, daher keine Berücksichtigung im Modell. }\end{array}$} \\
\hline
\end{tabular}

\section{5 Diskussion}

\section{Diskussion der Ergebnisse}

Die erste Forschungsfrage bezog sich auf die Relevanz der zusätzlichen Konstrukte aus der qualitativen Phase. In Analyse I zeigte sich sozioökonomische Problemwahrnehmung als positiver, Problemverneinung negativer Prädiktor für alle betrachteten Handlungsbereitschaften. Dass Wissen keinen oder einen nur geringen Einfluss auf Umwelthandeln hat, wurde durch zahlreiche Studien empirisch nachgewiesen (z.B. Bögeholz, 1999; Grob, 1991) und konnte durch die vorliegende Studie gestützt werden. Die 
höhere Relevanz der sozioökonomischen Problemwahrnehmung gegenüber einer ökologischen Problemwahrnehmung stützt eine zentrale Erkenntnis der qualitativen Phase der Studie (vgl. Abschnitt 1.3). Einschränkend muss in Bezug auf die zusätzlich eingeführten Konstrukte angemerkt werden, dass die Homoskedastizität in Bezug auf die Bereitschaft zu Aktivismus eingeschränkt war. Die Qualität des zu Grunde liegenden Regressionsmodells muss daher kritisch hinterfragt werden. In Analyse II hatten die auf der qualitativen Projektphase beruhenden Skalen keine Bedeutung (abgesehen von Problemverneinung).

In Hypothese 1 wurde konstatiert, dass, entsprechend Befunden aus der Literatur (Nordlund \& Garvill, 2002; Schultz \& Zelezny, 1999; Stern et al., 1993), die Werte des Clusters SelbstTranszendenz (Universalismus und Benevolenz) wichtigste Prädiktoren für eine Bereitschaft zum Schutz der Biodiversität seien. Diese Hypothese konnte teilweise gestützt werden. Der Wert Universalismus wies für alle betrachteten Handlungstypen den stärksten oder zweistärksten Einfluss auf. Benevolenz zeigte hingegen selbst in Analyse I keinen Einfluss. Es könnte daher sein, dass in Studien, in denen der Cluster Selbst-Transzendenz eingesetzt wurde entweder vor allem der Einzelwert Universalismus relevant war, oder in der vorliegenden Studie Varianzanteile von Benevolenz durch den Wert Universalismus übernommen wurden. Ein Indiz dafür ist die Korrelation zwischen den beiden Werten $(\mathrm{r}=.60)$.

In Hypothese 2 wurde eine höhere Bereitschaft von Jugendlichen postuliert, Biodiversität zu schützen, je stärker sie von ihrer Verantwortlichkeit für umweltschützendes Verhalten überzeugt sind. Diese Hypothese wird durch unsere Daten in Analyse I für alle erhobenen Handlungsbereitschaften, in Analyse II für die Handlungsbereitschaften nichtaktivistisches, öffentliches Handeln und Handeln im privaten Raum gestützt. Dieser Befund harmoniert mit Ergebnissen aus der Literatur, wo ebenfalls ein direkter Effekt des Konstrukts auf eine Bereitschaft zum Umweltschutz nachgewiesen werden konnte (Bamberg \& Möser, 2007; Guagnano et al., 1994; Kaiser \& Shimoda, 1999). Einzig für eine Bereitschaft zu Aktivismus hat sich Verantwortungszuschreibung und wahrgenommene Möglichkeit zu Handeln in Analyse II nicht mehr als relevant erwiesen. Stern et al. (1999) gaben in dieser Hinsicht zu bedenken, dass für eine Bereitschaft zu Aktivismus eine „environmentalist identity“ handlungssteuernd sein kann, was sich eher im Bereich der Werte und Persönlichen Normen niederschlagen würde. Für letzteres weist unsere Studie Evidenzen auf, da sich für die Bereitschaft zu Aktivismus vor allem Konstrukte aus den Bereichen der Werte und persönlichen Normen als relevant erwiesen haben.

Als Hypothese 3 wurde postuliert, dass Persönliche Normen einen großen Einfluss auf Bereitschaften von Jugendlichen haben, die Biodiversität zu schützen. Hypothese 3 kann gestützt werden. Persönliche Normen waren in beiden Analyseschritten höchst signifikante 
Prädiktoren, und zwar für alle betrachteten Handlungsbereitschaften. Zwar wies Universalismus für nicht-aktivistisches, öffentliches Handeln und Handeln im privaten Raum ein jeweils leicht höheres Betagewicht auf, doch sind auch hier Persönliche Normen starker Prädiktor.

In Bezug auf die zweite Forschungsfrage konnte festgestellt werden, dass sich neben denen unter Hypothese 1 und Hypothese 2 angenommenen Einflussfaktoren in Analyse II weitere Prädiktoren u.a. aus dem Bereich der Werte als relevant erwiesen haben. Der positive Einfluss des Werts Selbstbestimmung auf eine Bereitschaft zum Handeln im privaten Raum stützt Ergebnisse von Stern et al. (1999), die den Cluster Offenheit für Veränderungen (dem Selbstbestimmung angehört) als positiven Prädiktor für Umwelthandeln beschrieben haben. Eine Relevanz dieses Wertes gerade in Bezug auf Handeln im privaten Raum lässt sich möglicherweise durch die in den Items angeführten Handlungsmöglichkeiten erklären, die Jugendlichen Autonomieerleben ermöglichen. Beispiele sind Kaufentscheidungen gegen Produkte wie Kosmetika, die zur Gefährdung der Biodiversität beitragen können. Der Wert Stimulation gehört ebenfalls zu dem übergeordneten Cluster Offenheit für Veränderungen, das Bereitschaften zum Umweltschutz gemäß der Literaturlage positiv beeinflusst (Nordlund \& Garvill, 2002; Stern et al., 1999). Unser Ergebnis steht diesen Befunden entgegen. Im Kanon der im Fragebogen eingesetzten Items ist jedoch vorstellbar, dass Handlungsoptionen im privaten Raum Jugendlichen „bourgeois“ erschienen. Sie könnten besonders bei Befragten auf Ablehnung gestoßen sein, die, gemäß dem Wert Stimulation, ein aufregendes und abwechslungsreiches Leben schätzen. Für eine Bereitschaft zu Aktivismus hat sich der Wert Sicherheit als negativer Prädiktor erwiesen, was mit Befunden aus der Literatur harmonisiert (Stern et al., 1999). Es liegt nahe, dass beispielsweise Bereitschaften zum Anbringen eines Protesttransparents vor einer Firma oder das offensive Verteilen von Flugblättern auf Menschen mit einem hohen Sicherheitsbedürfnis keinen Reiz ausüben, selbst wenn eine grundsätzliche Bereitschaft zum Schutz der Biodiversität gegeben ist.

In Bezug auf die Gesamtstudie ist anzumerken, dass aufgrund der niedrigen Probandenzahl nur begrenzt verallgemeinerbare Aussagen getroffen werden können. Darüber hinaus setzte sich die Stichprobe aus Schüler(inne)n der Sekundarstufe II zusammen. Die Proband(inn)en verfügten somit über ein vergleichsweise hohes Bildungsniveau. In Bezug auf die Skalenkonstruktion ist weiterhin kritisch anzumerken, dass wenige Skalen im Original eingesetzt worden sind, was beim Vergleich mit anderen Studien in den Blick genommen werden muss. Die in dieser Studie eingesetzten Items reflektieren einen Bezug zur Lebenswelt und zu Handlungsmöglichkeiten junger Menschen im Kontext Biodiversität. Im Gegensatz zu einer allgemeinen Umweltschutzbereitschaft könnte die Zustimmung zu Handlungsbereitschaften in unserer Studie geringer sein. Der Grund für diese Annahme ist 
die hohe Komplexität des Themas Biodiversität und ein scheinbar geringerer unmittelbarer lokaler Bezug der Handlungsoptionen. Bei der Interpretation der Ergebnisse darf zudem nicht außer Acht gelassen werden, dass es sich bei allen drei abhängigen Variablen um Handlungsbereitschaften handelt. Tatsächliches Handeln zum Schutz der Biodiversität (wie beispielsweise Kaufentscheidungen in der vorliegenden Studie) mag in entscheidendem Maße externen Handlungsrestriktionen, wie fehlenden Anreizen (vgl. Hunecke, Matthies, Blöbaum \& Höger, 1999) oder Gewohnheiten (Klöckner \& Matthies, 2004) unterliegen.

Als Entwicklungsoption könnte das der Studie zu Grunde liegende Modell in seiner strukturellen Abfolge validiert werden. Vor allem Persönliche Normen könnten wiederum von im Modell distaleren Variablen beeinflusst werden, worüber Strukturgleichungsmodelle Aufschluss geben könnten. Bezüglich der Stichprobe könnte es im Zuge von internationalen Handlungserfordernissen aufschlussreich sein, Menschen aus unterschiedlichen kulturellen Kontexten einzubinden. Untersuchungen mit a) jüngeren Schüler(inne)n, b) Schüler(inne)n anderer Schulformen und c) Schüler(inne)n in Biodiversität-Hotspots könnten zu einer empirischen Basis für erfolgreiche Gestaltungsmöglichkeiten in der Umsetzung von Artikel 13a der CBD beitragen.

\section{Konsequenzen für die Weiterentwicklung von Biodiversitätsbildung}

Nach dem Stand der Umweltbildungsforschung reicht es nicht aus, ausschließlich kognitive Aspekte (wie beispielsweise Wissen) zu fördern, um Kompetenzen auszubilden. Nach Weinert sind Kompetenzen erforderlich, die er definiert als „die bei Individuen verfügbaren oder durch sie erlernten kognitiven Fähigkeiten und Fertigkeiten, um bestimmte Probleme zu lösen sowie die damit verbundenen motivationalen, volitionalen und sozialen Fähigkeiten und Fertigkeiten, um die Problemlösungen in variablen Situationen erfolgreich und verantwortungsvoll nutzen zu können.“ (Weinert, 2001, S. 23f.). Die Ergebnisse unserer Studie liefern erste Hinweise a) auf Anforderungen zur Lösung von Biodiversitätsproblemen und b) auf Faktoren, die vor allem für die Ausbildung von motivationalen und volitionalen Fähigkeiten im Bereich Biodiversität bedeutsam sein können.

Betrachtet man den starken Einfluss, den der Wert Universalismus für eine Bereitschaft von Oberstufenschüler(inne)n hat, die Biodiversität zu schützen, so wird deutlich, wie wichtig Bildungsmaßnahmen bereits im frühen Kindesalter sind: Werte werden früh angelegt und sind dann recht stabil (Schwartz, 1992). Dass eine emotionale und kognitive Beschäftigung mit heimischen Arten eine Wertschätzung der Biodiversität bei Kindern im Grundschulalter fördern kann, hat beispielsweise Lindemann-Matthies (2002) gezeigt. Aufgrund des starken Einflusses von Naturerfahrung auf Natur- und Umweltschutzhandeln (Bögeholz, 1999) ist 
denkbar, Bildungsmaßnahmen zur Naturerfahrung im Hinblick auf den Wert Universalismus auszugestalten. Der Wert Universalismus fasst u.a. die Wahrnehmung der Schönheit der Natur (Schwartz, 1992). Dieser Aspekt kann im Rahmen von Naturerfahrungen systematisch aufgegriffen werden. Ein weiteres zentrales Konstrukt war Verantwortungszuschreibung und wahrgenommene Möglichkeit zu Handeln. Für eine Biodiversitätsbildung unterstreicht dies die Wichtigkeit, reale Handlungsmöglichkeiten aufzuzeigen. Je überzeugter Jugendliche von der Wirksamkeit ihrer potenziellen Handlungen sind, desto eher werden sie gemäß unserer Ergebnisse bereit sein, Biodiversität aktiv zu schützen. Bei Bildungsmaßnahmen sollten daher gezielt Ansätze und Materialien zum Einsatz kommen, die auf eine kritische Reflexion von Handlungsmöglichkeiten zum Schutz der Biodiversität abzielen. Die Union of Concerned Scientists (Brower \& Leon, 1999) hat beispielsweise konkrete Vorschläge für ein reflektiertes Konsumverhalten vorgelegt, durch das mit einfachen Mitteln Ressourcen geschont werden können. Gute Ansatzpunkte bietet in dieser Hinsicht auch der „ökologische Rucksack“, der das „Gewicht“ an umweltschädlicher Last eines Produkts beschreibt (Schmidt-Bleek, 2004). Wenn Schüler(innen) mit Hilfe derartiger Ökobilanzen selbst zu dem Schluss kommen, dass ihr potenzieller Beitrag zum Schutz der Biodiversität tragfähig und wirkungsvoll ist, kann dies gemäß der vorliegenden Ergebnisse die volitionale Komponenten für eine Bereitschaft zum Schutz der Biodiversität fördern. Gleichzeitig werden Schüler(innen) für sozioökonomische Ursachen und Implikationen des Biodiversitätsverlusts sensibilisiert, was gemäß unserer Ergebnisse Bereitschaften zum Schutz der Biodiversität eher fördert als die Vermittlung ökologischen Sachwissens.

Zusammenfassend kann festgehalten werden, dass sich für die Umsetzung der CBD gezeigt hat, wie wichtig es ist, insbesondere an den Werten, Überzeugungen und Persönlichen Normen und einer sozioökonomischen Problemwahrnehmung von Schüler(inne)n anzusetzen, um eine Bereitschaft zum Handeln zum Schutz der biologischen Vielfalt zu fördern. Erforderlich sind langfristige Programme, die Biodiversitätsbildung kompetenzorientiert anlegen - und Programme, die sich nicht auf klassische ökologische Inhalte der Biodiversitätsproblematik beschränken. Unter Berücksichtigung der identifizierten Einflussvariablen sollten Lernumgebungen geschaffen werden, die es ermöglichen, neben kognitiven motivationale und volitionale Aspekte in die Bildungsarbeit einzubinden - mit dem Ziel, komplexe Probleme des Biodiversitätsverlusts sukzessive einzugrenzen. 


\section{Values, Beliefs and Norms That Foster Chilean and German Students' Commitment to Protect Biodiversity ${ }^{1}$}

\subsection{Abstract}

Based on the Value-Belief-Norm (VBN) theory, 15 to 19-year-old Chilean $(n=216)$ and German ( $\mathrm{n}=217)$ students' commitment to protect biodiversity was investigated. Comparisons revealed that Chilean adolescents showed higher personal norms and commitments to protect biodiversity. Regression analysis showed that within the German sample, the "Schwartz"-value universalism was an important predictor for three different kinds of behavioral commitment. In both samples, "ascription of responsibility", "perceived ability to reduce threats" and, above all, "personal norms" were positive predictors. The paper concludes with implications for biodiversity education, such as offering realistic behavioral options to protect biodiversity.

\section{$9.2 \quad$ Introduction}

Biodiversity loss is one of the most urgent challenges for sustainable development (WCED, 1987; UNCED, 1992; Wilson, 2001). Although biodiversity loss is a global phenomenon, socalled biodiversity hotspots show particularly serious extinction rates (Mittermeier et al., 2004). Central Chile is a region that contains such a hotspot. In order to prevent biodiversity loss, the Convention on Biological Diversity (CBD) emphasizes education as a central strategy for conserving biodiversity (UNCED, 1992). In response to this claim, several scientific studies have addressed the challenge of biodiversity education. For example, Lindemann-Matthies (2006) showed that a recognition of species diversity along children's school path significantly contributed to an appreciation of species diversity. Grace and Ratcliffe (2002) found that students had difficulties to integrate facts and values into their arguments when they talked about conservational issues. Gayford (2000) reported a tentativeness of teachers when having to teach socio-economic aspects of biodiversity. In support of the former, a qualitative study with German adolescents showed that an acknowledgement of economic and social aspects of biodiversity loss was a major challenge

\footnotetext{
${ }^{1}$ Source: Menzel, S., \& Bögeholz, S. (2007). Values, Beliefs and Norms That Foster Chilean and German Students' Commitment to Protect Biodiversity. Manuscript to be submitted for publication.
} 
for some students (Menzel \& Bögeholz, 2006). However, besides promoting a complete and therefore, interdisciplinary understanding of biodiversity and its loss, it is an important for biodiversity education to foster a commitment to protect biodiversity. In this regard, the question remained unanswered as to which factors influence young people's commitment to protect biodiversity. To shed light on this issue, German students' commitment to protect biodiversity was investigated in a questionnaire study (Menzel \& Bögeholz, 2006). The authors chose predictors from the Value-Belief-Norm (VBN) theory (Stern et al., 1995a; Stern et al., 1999; Stern, 2000) and combined them with scales that were retrieved from a former qualitative study (Menzel \& Bögeholz, 2005) reflecting ecological, economic and social problem perception, knowledge and problem denial. As a result, personal norms and a value orientation towards "universalism" (an affinity towards justice and appreciation of nature) proved to be highly relevant for predicting commitments to protect biodiversity. However, an intercultural perspective on students' commitment to protect biodiversity has not yet been taken. Nevertheless, such an approach could be useful for promoting a (global) biodiversity protection strategy in that it exemplarily reveals different perceptions of biodiversity and its loss. For example, in comparison with students living in industrial countries, students living in a biodiversity hotspot might have a different view on biodiversity and its loss. Particularly, according to theories of risk perception, commitments to protect nature (and influencing factors on such behavioral commitments) can vary between students who have a rather intellectual access to the problem (such as students living in an industrial country such as Germany) and those who can observe the biodiversity loss in their everyday-life (for an overview of theories of risk perception and their suitability for environmental problems see Gardner \& Stern, 1996).

\subsection{Theoretical framework}

Within the VBN theory, influencing factors on pro-environmental behavior are assumed on the levels of values, beliefs and personal norms. The inclusion of values, awareness of consequences and ascription of responsibility as beliefs and personal norms is based on the Norm-Activation-Model of Schwartz (1977) and Schwartz \& Howard (1981), which originally served to explain altruistic behavior. However, as the VBN theory explicitly aims at explaining pro-environmental behavior, a predictor is included that measures a general pro-environmental attitude (Stern et al. 1995b) namely, the New Ecological Paradigm (Dunlap et al., 2000). Within the VBN theory, altruistic, biospheric and egoistic value orientations can predict pro-environmental behavior (e.g. Stern et al., 1993; Stern et al., 
1995a, Stern et al. 1999, Stern, 2000). However, literature indicates a positive influence of biospheric and altruistic values which is shown to dominate (e.g. Guiterrez Karp, 1996; Schultz \& Zelezny, 1998; 1999).

"Beliefs" are composed of four different predictors: i) the New Ecological Paradigm (NEP, Dunlap et al., 2000), which is a general scale to measure a positive "folk" ecological theory about the relationship of man and nature (cf. Stern et al., 1995b) ii) an awareness of egoistic, altruistic and biospheric consequences of an environmental damage, iii) perceived ability to reduce threats and, iv) ascription of responsibility to protect nature.

Personal norms form the third component of the theory. According to the underlying NormActivation-Model, personal norms are strongly related to altruistic behavior in the VBN theory. However, such a strong relation has also been reported for pro-environmental behavior (e.g. Widegren, 1998; Stern et al. 1995a, Stern et al. 1999; Stern, 2000).

The VBN differentiates behavioral commitment as four types: i) activism, such as participating in public demonstrations, ii) non-activist, public sphere behavior such as signing a petition to demand nature protection, iii) private-sphere behavior, such as purchase decisions and iv) behavior in organizations; such as fostering recycling policies in the working environment.

\subsection{Research questions and hypotheses}

The aims of the paper are as follows: Firstly, Chilean and German students shall be compared in their beliefs, norms and different behavioral commitments to protect biodiversity. Secondly, relevant positive and negative predictors shall be identified that influence Chilean and German students' commitment to protect biodiversity. Our hypotheses and research question are as follows:

H1: Chilean and German students differ in an awareness of consequences, ascription of responsibility, personal norms and commitments to protect biodiversity.

H2: Personal Norms and the universal human value "universalism" are the strongest predictors for a commitment to protect biodiversity.

Besides these hypotheses, we formulated the following research question:

Q1: Which predictors are generally relevant to explain Chilean and German students' commitment to protect biodiversity? 


\subsection{Methods}

The VBN theory explains a general commitment to pro-environmental behavior. Therefore, we adjusted the model to the context of biodiversity (fig. 9.1). The model served as the basis for a questionnaire study.

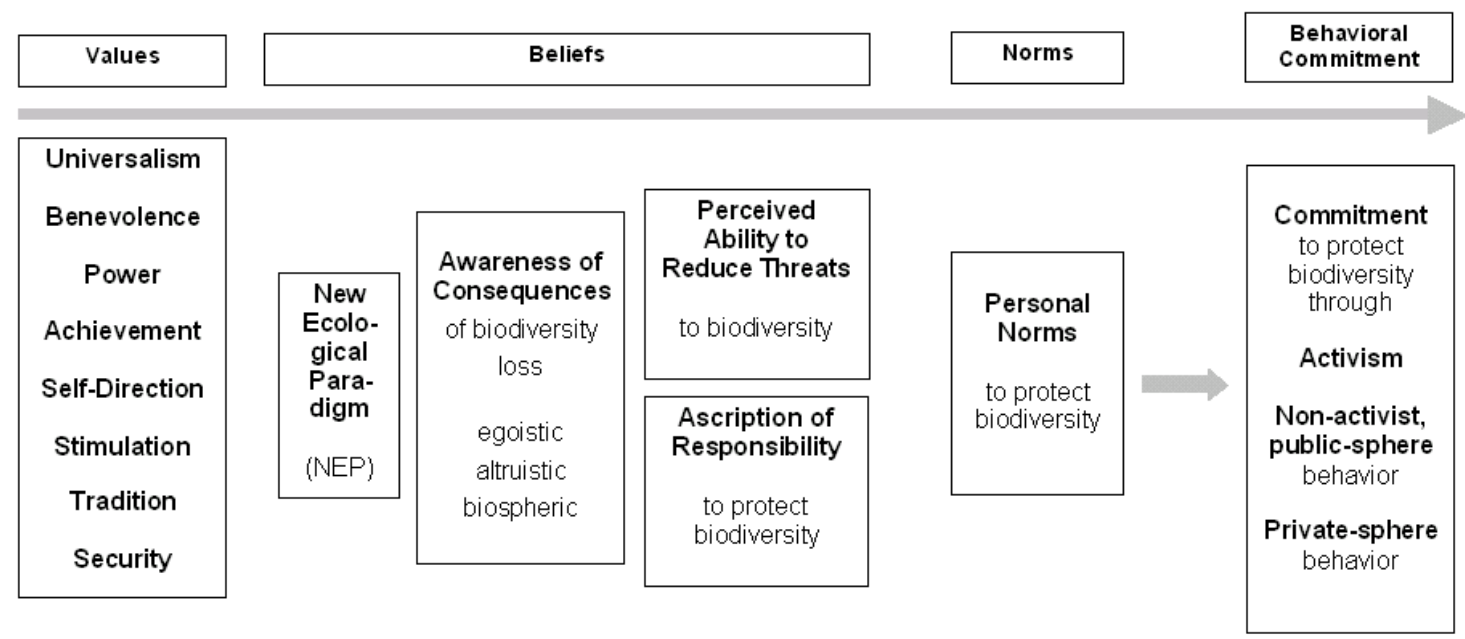

Fig. 9.1. Model to explain adolescents' commitment to protect biodiversity, based on the Value-Belief-Norm theory (Stern et al., 1995a; Stern et al., 1999; Stern, 2000).

The questionnaire was applied in November 2005 to 216 Chilean and in January 2006 to 217 German students aged 15-19. All German participants were enrolled in the $11^{\text {th }}$ grade of grammar schools, all Chilean students the "tercero medio" in secondary education respectively. In both countries, neither the subject "biodiversity" nor "ecology" had as yet been treated during their secondary education. For all predictors, we either drew scales from original literature or constructed scales after approved scales and adapted them to the context of biodiversity (cf. table 9.1). When necessary, scales were translated using the backtranslation method. Whenever no approved templates were available, we constructed new scales (see table 9.1). Values were assessed as ten universal human values after Schwartz (1992, 1994), eight of which are analyzed in the current paper. Regarding behavioral commitments, three commitment types were included in the analyses presented here: i) activism, ii) non-activist, public sphere behavior and iii) private-sphere behavior.

We assessed all variables through scales that contained a minimum of three Items. Alpha values ranged between 0,53 and 0,90 , except for the "Schwartz"-values tradition $(\alpha=0,48)$ and power $(\alpha=0,42)$ in the German sample, and for awareness of egoistic consequences in both samples ( $\alpha=0,40$ German sample; $\alpha=0,33$ Chilean sample). Basically, all applied scales were four-point Likert-scales that ranged from "I do not agree" to "I fully agree". An exception was the Portrait Value Questionnaire (PVQ, Schwartz, 2005) used for assessing 
value orientations. Here, a six-point Likert-scale was applied, in which students were asked to rate how much a hypothetic person is like them. Categories ranged from "is very much like me" to "is not at all like me".

Table 9.1. Sources of scales as used in the study.

\begin{tabular}{|l|l|}
\hline Scale & Sources \\
\hline Values & Portrait Value Questionnaire (PVQ) (Schwartz, 2005) \\
\hline New Ecological Paradigm & The Revised NEP Scale (Dunlap et al., 2000), translated \\
\hline Awareness of Consequences & Constructed after Stern et al. (1993; 1999) \\
\hline Perceived Ability to Reduce Threats & New construction \\
\hline Ascription of Responsibility & New construction \\
\hline Personal Norms & Constructed after Stern et al. (1999), Widegren (1998) \\
\hline Behavioral Commitment & Constructed after Stern (2000), Stern et al. (1999) \\
\hline
\end{tabular}

To compare the Chilean and the German sample, we calculated independent group t-tests although some variables did not follow a normal distribution. However, t-tests are relatively robust against broken assumptions, such as non-normal distributions (Delaney \& Vargha, 2000). Nevertheless, we validated our results with an U-Test. As a result, the same variables proved to be significant although effect sizes generally decreased. For the PVQ, Schwartz (2005) suggests the use of "centered values" whenever intercultural comparisons are intended in order to avoid cultural biases in the responses. Therefore, the scores for the PVQ were centered by subtracting the mean score of an individual across the entire value scale from each of the same individual's score for a single value (Schwartz, 2005).

In order to test hypothesis two (H2) and to answer the research question, we conducted regression analyses in two analytical steps. Within a first analysis (I), sets of predictors were used. First, we used all Schwartz values as predictors. Then, secondly, we used beliefs, and, within a third regression, personal norms as exclusive predictors for each type of behavioral commitment. In a second step (analysis II) only those predictors were included that had proven to be significant as a result of analysis I. We therefore constructed one regression model across all relevant predictors of the three predictor sets. In other words, all relevant values, beliefs, and personal norms were combined in one regression model for each type of behavioral commitment. With regard to the PVQ, raw scores were used for regression analyses, thus, means across all items of the respective value construct, as they were not compared cross-culturally. As a consequence, a potential bias would affect all scales of the study and therefore, not be relevant for the determination of relevant regressors. 


\subsection{Results}

Hypothesis one (H1) focused on a comparison of Chilean and German students. The results of independent group t-tests are reported in table 9.2.

Table 9.2. Comparison between mean scores of the German and Chilean sample.

\begin{tabular}{|lcccccc|}
\hline Predictor & $\begin{array}{c}\text { Mean } \\
\text { Chile }\end{array}$ & $\begin{array}{c}\text { SE } \\
\text { Chile }\end{array}$ & $\begin{array}{c}\text { Mean } \\
\text { Germany }\end{array}$ & $\begin{array}{c}\text { SE } \\
\text { Germany }\end{array}$ & T/p & $\begin{array}{c}\text { Effect size } \\
\text { d }\end{array}$ \\
\hline \multicolumn{6}{c}{ Values } & MRATS - centered values \\
\hline Self-Direction & 0.46 & 0.04 & 0.60 & 0.05 & $2.13^{*}$ & 0.21 \\
\hline Power & -0.89 & 0.07 & -0.95 & 0.07 & -0.60 n.s. & -0.06 \\
\hline Universalism & 0.47 & 0.04 & 0.20 & 0.06 & $-3.79^{* * *}$ & -0.37 \\
\hline Achievement & 0.03 & 0.06 & 0.03 & 0.06 & -0.05 n.s. & -0.01 \\
\hline Security & -0.18 & 0.05 & -0.04 & 0.05 & 1.90 n.s. & 0.18 \\
\hline Stimulation & 0.14 & 0.05 & 0.17 & 0.06 & 0.31 n.s. & 0.03 \\
\hline Tradition & -0.39 & 0.05 & -0.78 & 0.06 & $-4.65^{* * *}$ & -0.45 \\
\hline Benevolence & 0.46 & 0.04 & 0.55 & 0.05 & 1.36 n.s. & 0.13 \\
\hline & Beliefs, mean scores, based on 4-pt. Likert Scales & & \\
\hline NEP & 1.92 & 0.03 & 2.11 & 0.03 & $5.10^{* * * *}$ & 0.49 \\
\hline $\begin{array}{l}\text { Awareness of Egoistic } \\
\text { Consequences }\end{array}$ & 1.34 & 0.04 & 0.98 & 0.03 & $-7.34^{* * *}$ & -0.71 \\
\hline $\begin{array}{l}\text { Awareness of Altruistic } \\
\text { Consequences }\end{array}$ & 2.06 & 0.03 & 1.98 & 0.04 & -1.60 n.s. & -0.15 \\
\hline $\begin{array}{l}\text { Awareness of } \\
\text { Consequences for the } \\
\text { Biosphere }\end{array}$ & 2.49 & 0.03 & 2.32 & 0.03 & $-3.53^{* * * *}$ & -0.34 \\
\hline $\begin{array}{l}\text { Perceived Ability to } \\
\text { Reduce Threats }\end{array}$ & 2.19 & 0.04 & 1.76 & 0.05 & $-6.89^{* * *}$ & -0.66 \\
\hline $\begin{array}{l}\text { Ascription of } \\
\text { Responsibility }\end{array}$ & 2.14 & 0.04 & 1.69 & 0.05 & $-7.52^{* * * *}$ & -0.72 \\
\hline & 1.67 & 0.05 & 1.10 & 0.05 & $-7.67^{* * * *}$ & -0.74 \\
\hline Personal Norms & & & & & \\
\hline
\end{tabular}


Table 9.2. (continued)

\begin{tabular}{|c|c|c|c|c|c|c|}
\hline Predictor & $\begin{array}{l}\text { Mean } \\
\text { Chile }\end{array}$ & $\begin{array}{c}\text { SE } \\
\text { Chile }\end{array}$ & $\begin{array}{c}\text { Mean } \\
\text { Germany }\end{array}$ & $\begin{array}{c}\text { SE } \\
\text { Germany }\end{array}$ & $\mathbf{T} / \mathbf{p}$ & $\begin{array}{l}\text { Effect size } \\
\quad d\end{array}$ \\
\hline \multicolumn{7}{|c|}{ Behavioral Commitment, mean scores, based on 4-pt. Likert Scales } \\
\hline Activism & 1.72 & 0.05 & 0.78 & 0.05 & $-12.19 * * *$ & -1.18 \\
\hline $\begin{array}{l}\text { Non activist, Public- } \\
\text { Sphere Behavior }\end{array}$ & 1.97 & 0.05 & 1.45 & 0.05 & $-7.40 * * *$ & -0.71 \\
\hline Private-Sphere Behavior & 2.02 & 0.05 & 1.44 & 0.06 & $-7.50 * * *$ & -0.73 \\
\hline
\end{tabular}

Three values differed significantly between the two groups, even though effect sizes were small. The values were self-direction, universalism, and tradition. Note that Chilean students scored higher on universalism and tradition, while German students scored higher on selfdirection. Regarding beliefs, all variables differed significantly, apart from awareness of altruistic consequences. Chilean students scored higher on all scales, except for the NEP. Differences showed medium effects, apart from the NEP and an awareness of consequences for the biosphere, where effect sizes were found to be smaller. Regarding personal norms and the three types of behavioral commitment, the Chilean students scored significantly higher. Effect sizes were medium throughout, and large in the case of activism.

A second focus was the identification of predictor sets with the overall aim to explain Chilean and German students' commitment to protect biodiversity through activism, non activist, public-sphere behavior and private-sphere behavior. The results of the regression analyses (analysis I and analysis II) for the Chilean sample are depicted in table 9.3 and for the German sample in table 9.4 respectively.

For the Chilean sample, personal norms proved to be the most important predictor for all three types of commitment to protect biodiversity. Security and ascription of responsibility had a positive influence on a commitment to activism besides personal norms. In contrast, for non activist, public-sphere behavior, the value tradition was the only positive predictor besides personal norms. For a commitment to private-sphere behavior, the NEP as well as a perceived ability to reduce threats both had a positive influence.

In the German sample, personal norms were highly significant for each type of commitment to protect biodiversity. However, the universalism value showed a similar high relevance and was the strongest predictor for non activist, public-sphere and private-sphere behaviors. For the commitment to activism, security and ascription of responsibility were relevant predictors besides universalism and personal norms (note that security had a negative influence). 
Table 9.3. Results of regression analyses for the Chilean sample $(\mathrm{n}=216)$. Predictors for analysis II were selected as a result of analyses I, where regressions were conducted separately by values, beliefs, and personal norms. $\mathrm{AC}=$ Awareness of Consequences; $\mathrm{NEP}=$ New Ecological Paradigm. All values (except shaded) are standardized $\beta$-values.

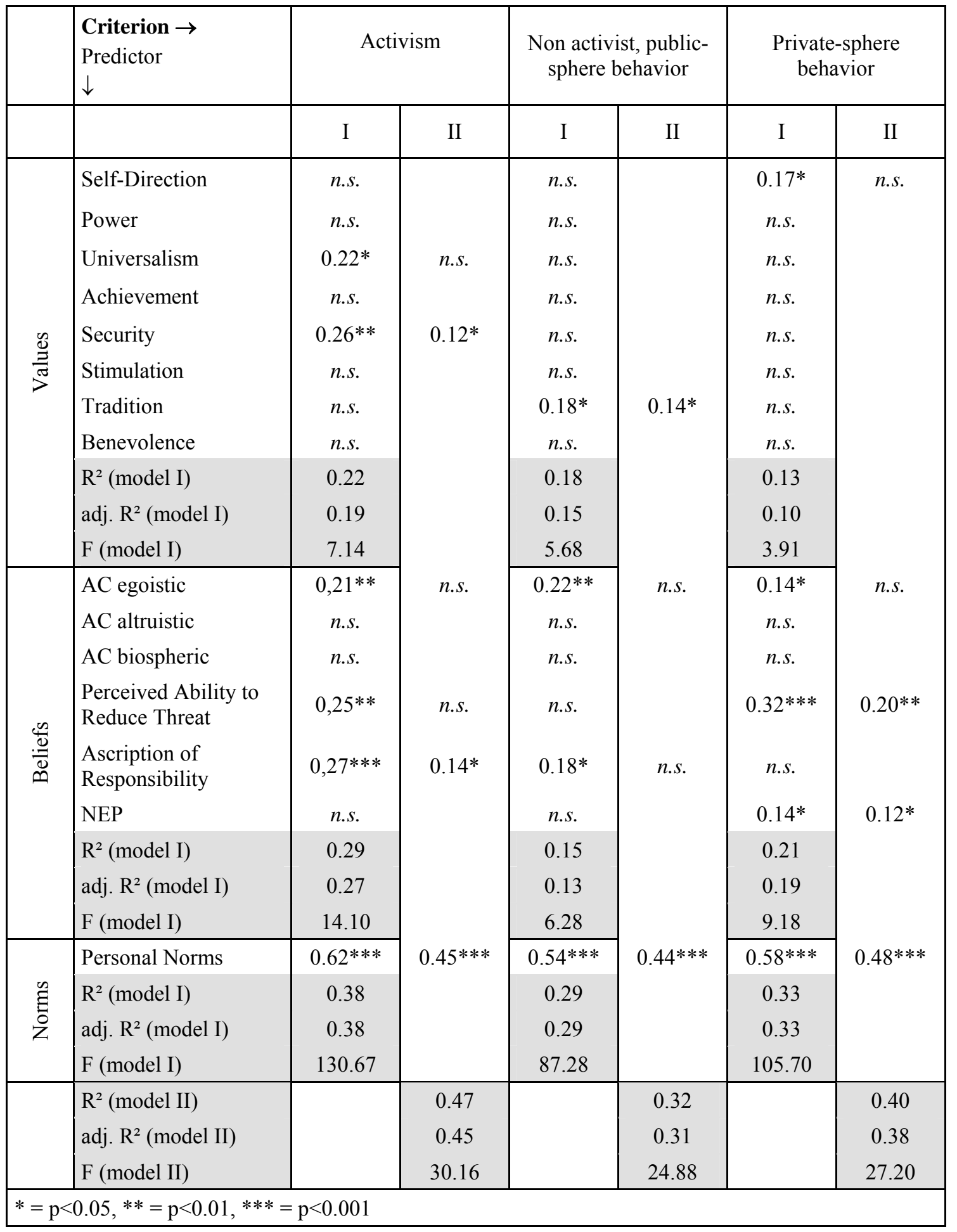

To explain non activist, public-sphere behavior, only the NEP proved to be relevant with a positive influence apart from personal norms and universalism. Five predictors explained private-sphere behavior. Universalism and self-direction had a positive influence, while 
stimulation served as a negative predictor. Apart from personal norms, perceived ability to reduce threats to biodiversity was the fifth predictor for this commitment type, which again was found to be positive.

Table 9.4. Results of regression analyses for the German sample $(\mathrm{n}=217)$. Predictors for analysis II were selected as a result of analyses I, where regressions were conducted separately by values, beliefs, and personal norms. AC $=$ Awareness of Consequences; NEP $=$ New Ecological Paradigm. All values (except shaded) are standardized $\beta$-values.

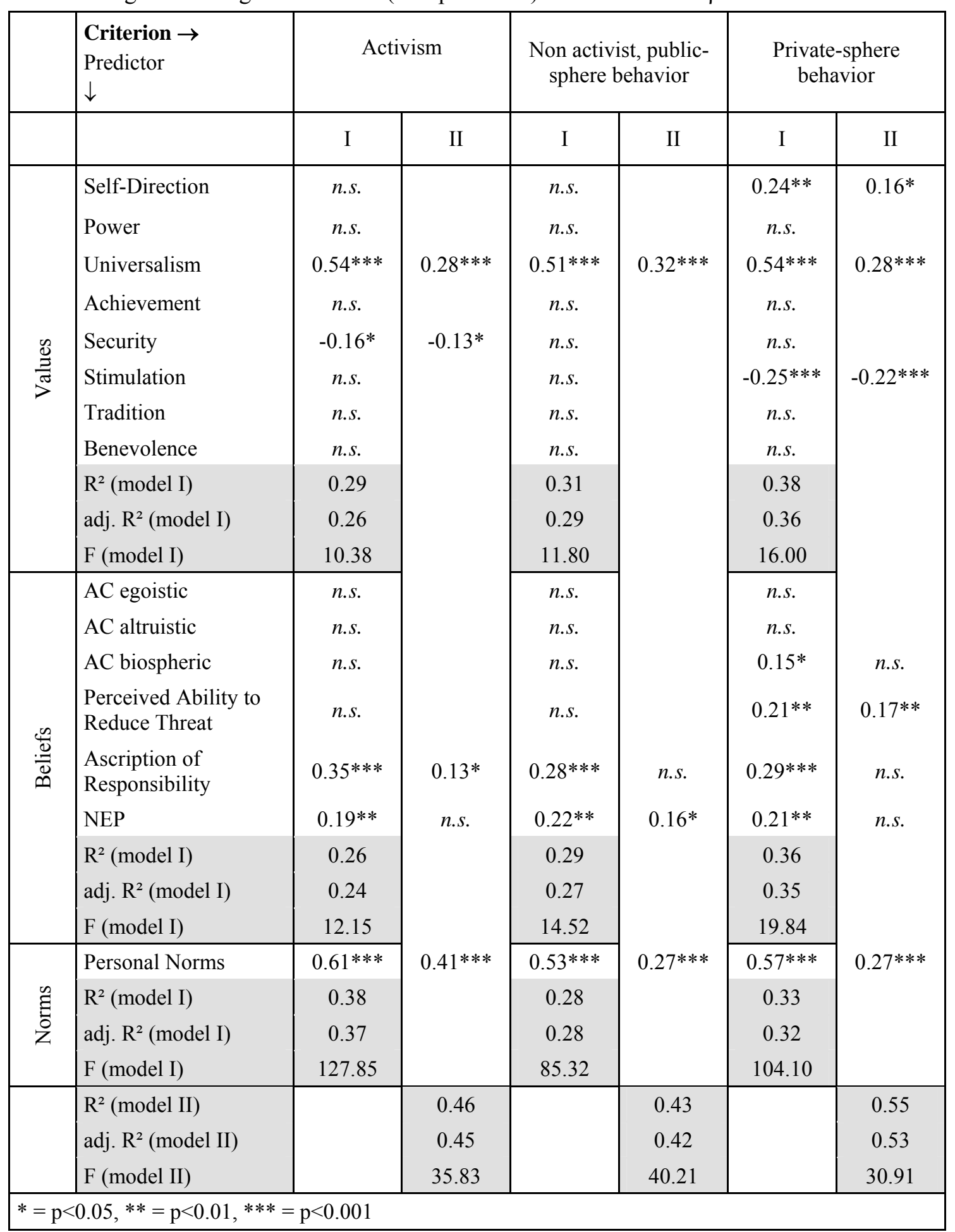




\subsection{Discussion}

In the first hypothesis we stated that Chilean and German students differ with regard to awareness of the consequences of biodiversity loss and ascription of responsibility to protect biodiversity, personal norms and behavioral commitments, due to Chileans' proximity to the problem. Our data supported this hypothesis partially. Interestingly, Chilean students responded more positively on almost all scales relevant to hypothesis 1. Exceptions were awareness of altruistic consequences (n.s.) and the NEP, in which German students scored higher.

Regarding the lower scores of Chilean students on the NEP, some authors report similar findings with Latin American samples (cf. Johnson et al., 2004). However, some authors stated that people with a Latin American cultural background are more connected with nature (Lynch, 1993) or more "ecologically attuned" (Noe and Snow, 1990) and also score higher on the NEP (e.g. Schultz et al., 2000).

The higher scores of Chilean students for ascription of responsibility, awareness of consequences, personal norms and for the three types of behavioral commitment could be due to Chileans' proximity to the problem of biodiversity loss. Direct observation of biodiversity loss might lead to a stronger personal norm and therefore, a higher commitment to becoming active in environmental protection. However, environmental protection is only slowly gaining public interest in most Latin American countries including Chile (Rieckmann, 2004). Therefore, we should consider that Chilean students might not see the actual efforts to be invested for pro-environmental behavior. In contrast, German students might be desensitized of environmental protection and other problems, such as unemployment superpose environmental issues on students' list of concerns (Hurrelmann, Albert \& TNS Infratest, 2006). The extremely low commitment of German students to protect biodiversity through activism supports the finding of a recent Greenpeace study which repeated that young Germans prefer private activities such as purchase decisions (Greenpeace, 2005). For Chilean students the same might be true in that although a commitment to protect biodiversity through activism was clearly higher in the Chilean sample, activism was again the type of commitment that displayed the lowest approval.

In hypothesis 2 we assumed that universalism and personal norms would be the strongest predictors for all assessed behavioral commitments to protect biodiversity. However, hypothesis 2 can only be supported partially. On the one hand, personal norms were extremely important for predicting all commitment types in both samples. Within the Norm- 
Activation Model, personal norms are defined as, "self-expectations for specific actions in particular situations that are constructed by the individual " (Schwartz, 1977, p. 227). It is evident that such a self-expectation is an important precondition for showing proenvironmental behaviors, a result that has also been reported in other studies (cf. Stern et al., 1995a; Widegren, 1998; Stern et al., 1999; Stern, 2000). On the other hand, only the universalism value played a central role in the German sample. A positive influence of a cluster of Schwartz values that universalism belongs to has been shown for proenvironmental behavior (e.g. Gutierrez Karp, 1996; Schultz \& Zelezny, 1998) as well as for pro-environmental attitudes (e.g. Schultz \& Zelezny, 1999). This result is understandable given that the universalism value embodies elements "justice", and "respect and appreciation of nature". However, in the Chilean sample, universalism does not play a major role. Therefore, other values might be of greater importance to young Chileans, such as security for activism or tradition for non activist, public-sphere behavior.

With respect to our research question, different predictors proved to be relevant besides universalism and personal norms and for the different types of behavioral commitment in the two samples. Apart from universalism, the security value played a negative role with respect to activism in the German sample. A possible explanation is that those students who appreciate a secure life situation would rather not participate in potentially dangerous activities such as tagging a protest banner to the outside of a company. The positive role of self-direction for a commitment to private-sphere behavior indicates that the protection of biodiversity in the private sphere might serve as an expression of individualism. Possibly, students appreciate to make their own choices, which in turn, serves as a motivation for biodiversity protection. In contrast, the negative influence of stimulation on private-sphere behavior is more difficult to explain. In this regard, some German students may think of some behaviors to protect nature in order to be bourgeois. Those who appreciate a stimulating life would then probably avoid behaviors that they consider to be bourgeois or narrow-minded. Regarding beliefs, the NEP and ascription of responsibility played a significant role for each behavioral type in the German sample, at least in analysis I. A general conviction of humans' and nature's interdependence as expressed by the NEP has been described as being conductive for a commitment to protect nature (e.g. Schultz \& Zelezny, 1998; Stern et al., 1995b; for an overview see Dunlap et al., 2000, p. 429). Ascription of responsibility as a positive predictor can be explained by the logic that those who feel responsible for the protection of biodiversity will also show a higher commitment to do so. Especially with respect to activism, this feeling of responsibility is important for a commitment to protect biodiversity. 
The Chilean students' commitment to non-activist, public sphere behavior is besides personal norms predicted by the tradition value. Nevertheless, tradition has been described as a negative predictor for pro-environmental behavior (Stern et al., 1995a), which contradicts our finding. Our results suggest that certain traditional values in Chile foster a commitment to protect biodiversity.

A commitment to private-sphere behavior by Chilean students can be predicted by personal norms, perceived ability to reduce threat and ascription of responsibility. It therefore seemed as if realistic options to contribute to biodiversity protection are important for fostering a commitment to private-sphere behavior. Moreover, the positive influence of security on the commitment to activism is interesting, as it served as a negative predictor in the German sample. This finding suggests that Chilean students probably note a risk of security through biodiversity loss, which in turn, may motivate a commitment to this radical form of behavior to protect biodiversity. Besides that is the fact that an awareness of egoistic consequences was relevant for all types of behavioral commitment in analysis I suggests the same. In the Chilean sample, a personal consternation seemed to be more important than in the German sample.

Although our study suggests that predictors from the VBN theory are suitable for predicting commitments to protect biodiversity in the present samples, some critical aspects should be considered. Firstly, it should borne in mind that the samples are not representative, neither for Chilean nor German society, nor for students from the two countries. All students were enrolled in secondary education. Therefore, all test persons had access to higher education.

Secondly, it is arguable whether personal norms superceded weaker predictors due to their strong influence. We tried to address this problem by first conducting regressions for each block in analysis I. However, it would be interesting to identify predictors for personal norms for the purpose of investigating influencing factors on the important predictor of personal norms.

A third critical point is a potential cultural bias reflected by the positive response tendency of the Chilean sample. However, as Chilean scores were lower (e.g. for the NEP) or not significantly higher on some constructs (e.g. for an awareness of egoistic consequences), it is more likely that positive responses actually reflect a higher concern and commitment on behalf of the Chilean students. Overall, despite these potential shortcomings, important educational implications can certainly emerge from the results. 


\subsection{Conclusions and implications for education}

According to our data, the most promising strategy for fostering a commitment to protect biodiversity would be the strengthening of personal norms. However, the strong relationship between personal norms and behavioral commitments suggests that influencing factors for both norms and commitment could be similar. Therefore, in order to deduce educational implications, it is useful to consider the values and beliefs which proved to be relevant.

The central motive for Chilean students seemed to be their personal concern and dismay. It would therefore be helpful to point out potential consequences of biodiversity loss on a local level. However, a perceived ability to reduce threats should be encouraged in parallel to developing realistic behavioral perspectives, especially for private-sphere behavior. Such educational measures could, once students notice what they can contribute, at the same time contribute to an enhancement of an ascription of responsibility and perceived ablitity to reduce threats to biodiversity, which is also important for a commitment to private-sphere behavior.

Human values are stable and difficult to change. Therefore, for the German sample, we should bear in mind that universalism cannot be actively taught. However, biodiversity education could build upon the universalism value by focusing on aspects such as justice and solidarity. These are important questions surrounding the topic of biodiversity, especially when the use and overuse of natural resources are picked out as a theme for curricula. Furthermore, in the German sample, an ascription of responsibility also proved to be important, at least based on the findings from analysis I. It is therefore important for both Chilean and German students to foster their awareness for the own responsibility for biodiversity conservation. Examples of well-reflected purchase decisions are suggested by Brower and Leon (1999) and Davidson \& Hatt (2005). For biodiversity education, it would be a valuable starting point to exemplify concrete and effective behavioral perspectives for fostering young people's feelings of responsibility can really contribute to sustaining biodiversity. 



\section{Summary and Discussion}





\section{Summary and Discussion}

\section{Pupils' Cognitive Frameworks and their Commitment to Protect Biodiversity}

Within this final part of the dissertation, an overall summary and discussion of central research findings is provided. Chapter 10.1 is a summary and discussion of the qualitative investigation of Chilean and German pupils' cognitive frameworks (research focus I). The same approach follows for research focus II in that a summary and discussion of fostering and inhibiting factors on Chilean and German pupils' commitment to protect biodiversity is provided (chapter 10.2). Chapter 10.3 is an overall critical discussion of the study. The dissertation closes with the most central educational implications of the study and concluding remarks (chapter 11).

\subsection{Cognitive frameworks of pupils in Chile and Germany (research focus I)}

In the following chapter, overall research questions, as raised in the introductory chapter with regard to Chilean and German pupils' cognitive frameworks, will be addressed in the form of a synopsis of research outcomes and a discussion.

(Q 1.1) Which associations and concepts can be identified with young people from Chile and Germany that either foster or oppose an understanding of biodiversity and resource dilemmas?

With regard to this research question, two chapters provided the first overview of pupils' ideas on biodiversity (chapters four and five). The two subsequent chapters went in greater detail in that cognitive frameworks were first explored and then, whenever possible, assigned to different levels of complexity, namely; associations, concepts and subjective theories (chapters six and seven). 
To sum up the relevant findings for this first overall research question, the following should be resumed:

(1) The term biodiversity was unknown to all German pupils within the qualitative sample, whilst all Chilean interviewees rightly associated the "variety of species" with the term "biodiversity". In contrast, all pupils across both samples interpreted the term biological diversity as "variety of species". Generally, pupils of both samples tended to concentrate on the species level of biodiversity. However, although less numerous, associations regarding the ecosystems level were identified, while the recognition of the genetic level of biodiversity was challenging for the interviewees. Two pupils, nevertheless, provided an interesting concept of "genetic diversity as an insurance for survival under changing conditions".

(2) Cognitive frameworks on values of biodiversity were identified on the levels of concepts and associations. Besides the above-mentioned concept of a value of genetic diversity as an insurance for survival under changing conditions, a number of associations regarding human-centred values of biodiversity occurred within both samples, such as biodiversity as sources of food, medicine, or raw materials.

(3) Two prominent concepts that served to explain low biodiversity in certain regions, as well as biodiversity loss, emerged across both samples. The first concept was one of "lack of space", which explained low (or threats to) biodiversity due to the lack of space in cities and other human-dominated places. The second concept was one of “extreme climatic conditions". Here, regions with naturally occurring climatic extremes were regarded as being unsuitable for high biodiversity.

Chilean pupils clearly seemed to be more familiar with the term "biodiversity". However, it is difficult to trace why the term was better known amongst Chileans. As a matter of course, it is possible that Chilean pupils had already been in touch with the topic and were, therefore, better informed. However, the topic "biodiversity" had not been treated in detail in any of the interviewees' schools. Presumably, there is another explication for the phenomenon. The Spanish term "diversidad" (diversity) is more commonly used in everyday language. The term appears in different contexts and is not restricted to scientific discourses, which is the same in the English language. In contrast, the German term "Diversität" (diversity), which forms a part of the term "Biodiversität" (biodiversity) is not used in common language and may, therefore, appear as a foreign word. This problem was less likely to occur with the term "biological diversity". The German translation is "biologische Vielfalt". "Vielfalt" can also 
be translated as "diversity", but, in contrast to "Diversität", it is a term that is commonly used in everyday speech. Accordingly, pupils of both countries rightly associated the variety of species with the term. However, the exclusive interpretation of "biodiversity" and "biological diversity" as the variety of species has shortfalls in that it does not describe the biodiversity concept in its full extent (cf. CBD, 1992, Art. 2.; Kerski \& Ross, 2005; Pullin, 2002). In this regard, the results showed that, for most pupils, a spontaneous association with the two other levels of the biodiversity concept (diversity of ecosystems and genetic diversity) was challenging. With regard to the level of ecosystems, however, the situation seemed to be slightly less difficult as here, in contrast to the level of genetic diversity, associations or concepts were shown within both samples.

With respect to values of biodiversity, numerous examples of human-centred (anthropocentric) values occurred. Many pupils of both samples saw biodiversity as a source of food and medicine and other commodities for humans. Such associations remind one of an economic perspective on biodiversity that pronounce instrumental values (Marggraf \& Streb, 1997). However, during the interviews such associations with instrumental values of biodiversity can be partly ascribed to products that were presented to the interviewees in the initial step of the interview (see fig. 7.1). However, even before the presentation of pharmaceuticals, teas and other herbal products, pupils referred to biodiversity as something that humans can profit from. Additionally, Helmers (2004) reported a focus on anthropocentric values within the pilot phase of this study, with different pupils. In contrast, an intrinsic value of biodiversity was seldom explicitly mentioned. The concept of genetic diversity as an insurance of survival under changing conditions, though, possibly takes a direct value for nature itself into account. Besides this rare concept, some pupils mentioned that high biodiversity is important for the respective ecosystem they belong to. The concept of "value of biodiversity for the respective ecosystem they belong to" has again also been identified by Helmers (2004). Therefore, an intrinsic value of biodiversity was at least singularly considered. After all, it is difficult to decide whether pupils thought that a survival of ecosystems would again be important for humans or, in contrast, for nature itself. Only the latter would actually reflect an intrinsic value of biodiversity. However, in environmental ethics it is argued whether such an intrinsic value really exists as all values that humans attribute to nature must in its final logic be anthropocentric (Krebs, 1997).

One central goal of biodiversity education is to foster biodiversity conservation (CBD, 1992). In this regard, pupils' ideas concerning reasons for biodiversity loss are particularly interesting. Two concepts emerged from the interviews that addressed the topic of biodiversity loss. Firstly, we should note that pupils spontaneously considered human influence on biodiversity loss, which became apparent through the concept of "lack of 
space". In particular, the concept expressed a notion of occupied space by human activity, due to the expansion of industrial estates, road networks and also of agricultural areas. However, pupils who showed this prominent concept seemed to have a very practical perspective on biodiversity loss. On the one hand, the concept embodied realistic perspectives because habitat destruction is, in fact, a serious threat to biodiversity (Pullin, 2002; Hilty, Lidicker \& Merenlender, 2006), internationally as well as in the European context. On the other hand, the concept led to a simplified perspective for two reasons. Firstly: habitat destruction is only partly due to the presence of urban structures and agricultural monocultures. Over all, effects are not as linear as expressed by the concept in that habitat destruction does not usually happen as a "side-effect" of human development in industrial countries as was often expressed within the concept. Mostly, multiple effects, often of global dimension, lead to habitat destruction rather than being a local effect "on the spot" (consider the spatial trap of resource dilemmas (Ernst, 1997), as presented in chapter 2.3). Secondly, the concept of "lack of space" concentrated on urban areas in Europe and North America. For instance, industries or building development in the outskirts of cities were blamed for occupying too much space. Therefore, in most cases, biodiversity loss in socalled developing countries was not considered. However, the phenomenon of habitat destruction is first and foremost a problem of developing countries (Hilty et al., 2006; Pullin, 2002; Wilson, 2001).

The second prominent concept that addressed reasons for low biodiversity and biodiversity loss respectively, focussed on extreme climatic conditions. Although it was slightly less prominent than the concept of "lack of space", it occurred repeatedly within both samples. Pupils who showed this concept mentioned the Poles or deserts as regions of low biodiversity and they named drought, heat, or cold as limiting factors to biodiversity. However, the concept of extreme climatic conditions is opposed to the scientific fact that high biodiversity often occurs in regions that actually show climatic extremes. Examples are the poor soils of many tropical rainforests (Milliken \& Ratter, 1998), droughts in the deserts - like the hotspot "Succulent Karoo" (Mittermeier et al., 2004) - and extremely low rates of nutrients in the water of coral reefs (Hallock \& Schlager, 1986; Stanley, 2006). The concept does not acknowledge the fact that, during evolution, poor environmental conditions often lead to extremely high biodiversity through concurrence, consequent mutation pressure, symbioses and occupation of ecological niches (e.g. Mayr, 2002; Townsend et al., 2003; for coral reefs cf. Stanley, 2006). Therefore, the concept could be misleading in both the understanding of biodiversity loss and of evolutionary roots of high biodiversity. Unfortunately, the concept may be enhanced by the public debate on climate change. In fact, climate change might be a threat to biodiversity (Flenley \& Bush, 2006; Lovejoy \& Hannah, 
2006; Pounds et al., 1999). Although climate change and climatic extremes are not directly linked under all circumstances, in public discussion, climate change is often equalised with emerging climatic extremes. Thereby, pupils' concept of climatic extremes as a threat to biodiversity might be supported. However, the climate itself is not the problem. Rather, the rapid climate change puts biodiversity at risk in that some plants, animals and ecosystems are not able to adapt quickly enough to newly emerging climatic conditions (Pounds et al., 1999).

(Q 1.2) Can types be identified across both samples that reflect prominent subjective theories that are opposed or fostering for an understanding of biodiversity and biodiversity loss?

The most central research findings relevant to this question were the following:

(1) Across the two samples, three subjective theories could be identified, namely; "loss of biodiversity due to ecological reasons", "loss of biodiversity due to economical reasons", and "loss of biodiversity due to social reasons".

a. The subjective theory "loss of biodiversity due to ecological reasons" mainly considered ecological reasons for, and consequences of, biodiversity loss. However, with regard to ecological facts, pupils often argued in a simplified and/or incorrect manner. Moreover, pupils who mainly considered ecological aspects of biodiversity loss, tended to display negative judgement about people in resource dilemmas.

b. A second subjective theory became apparent within the Chilean sample which was labelled "loss of biodiversity due to economical reasons". Here, pupils ascribed reasons for biodiversity loss to economic forces, mostly on the level of the individual's or industry's greed for money.

c. The subjective theory "loss of biodiversity due to social reasons" enabled pupils to integrate social aspects with reasons for and consequences of biodiversity loss. Moreover, pupils who showed this subjective theory, argued in a more balanced manner with respect to ecological, economic and social aspects of biodiversity loss.

(2) All subjective theories could be identified in more than one pupil. Therefore, the above-mentioned subjective theories served as a basis for a typology across both samples. In accordance with the underlying subjective theories, three types were identified: an ecological type, an economical type, and a balanced type. The latter 
was based on the subjective theory, "loss of biodiversity due to social reasons", while the two other types were based on the respective subjective theories that showed an ecological or economic focussing. In total, the subjective theory "loss of biodiversity due to ecological reasons" was the most prominent theory across both samples.

Subjective theories are the most complex level of cognitive frameworks and they are often implicit (e.g. Groeben et al., 1988; Piaget, 1974; Posner et al., 1982). Nevertheless, the power of such frameworks, particularly if one is not aware of them, can be extraordinary. Hence, in order to demonstrate how inhibiting inflexible cognitive frameworks can be for the understanding of new contents, an example will be instanced. Consider the following famous "brain twister":

A man and his son are driving in a car. The car crashes into a tree, killing the father and seriously injuring his son. At the hospital, the boy needs to have complicated brain surgery. Upon looking at the boy, the specialist surgeon says (telling the truth), "I cannot operate on him. He is my son." (unknown author, cited after Kilchör, 2001)

Although the solution to this is simple, most people come up with rather farfetched ideas instead, such as the son being the doctor's illegitimate child, rather than considering the obvious (the doctor is the boy's mother). Why is that so? Possible explanations are unconscious cognitive frameworks that oppose the idea that a doctor, obviously successful in that she is capable to conduct complicated brain surgery, can be a woman. Although this example is often used in the context of exploring unconscious sexist stereotypes - which is not the topic of this dissertation - the example demonstrates impressively how certain cognitive structures can guide thinking paths - and therefore, argumentation strategies when we talk about an issue.

Within the subjective theories that were presented in chapters six and seven, a comparatively strong influence of underlying subjective theories came to the fore. The statements of the German pupil, Natascha, as presented in chapter seven are examples of this. Natascha had a noticeably strong subjective theory that biodiversity and biodiversity loss are biological issues. Therefore, she obviously concluded that biodiversity loss must be a purely ecological phenomenon. As a consequence, Natascha tended to apply farfetched ideas, such as prevailing climatic circumstances, to explain the phenomenon of biodiversity loss in accordance with her obviously robust subjective theory. Although social and economic information was presented to her within the interview, she was not able to integrate this 
information. The strategies therefore, of which Natascha's responses serve as an example, remind one of the common answers to the brain twister about the woman surgeon. In both examples, the obvious is not assumed since an implicit, and yet powerful, subjective theory is too restricted to include the newly presented information in its full width. Cognitive frameworks are a result of individual experiences. Thus, according to a constructivist perspective, each individual has his or her own subjective view of the world (Piaget, 1974; von Glasersfeld, 2005). However, especially for teaching practices, it can be useful to identify subjective theories that are prominent and that therefore occur in more than one pupil. With regard to this, Kattmann, Gropengießer \& Komorek (1997) assumed that some subjective theories exist in more than one pupil when they are assessed with pupils who have comparable learning backgrounds. We therefore set up a typology based on prominent subjective theories.

The ecological type was the most prominent across both samples. In contrast, fewer pupils could be assigned to the balanced type. The economical type only emerged from an analysis of both samples. In the German sample, the underlying subjective theory "loss of biodiversity due to economic reasons" did not emerge clearly.

Two negative consequences for an understanding of biodiversity arose with regards to the ecological type. Firstly, our data showed that a one-dimensional ecological consideration of biodiversity and biodiversity loss fostered a tendency to invent ecological explanations that were often incorrect. As a consequence, less successful understanding of both the concept of biodiversity, in general, and ecological interrelations arose. Secondly, little (or no) empathy was shown towards people in resource dilemmas. The consequence of the latter tendency was an unjust attribution of responsibility to plant collectors, who were exclusively blamed for the destruction of natural resources. This, however, reflects by no means the real structure of resource dilemmas (Ernst, 1997; Hardin, 1968). Pupils of the economic type, on the one hand, succeeded in including economic aspects when talking about biodiversity and biodiversity loss. However, arguments were sometimes naïve in that they described people's (or the industry's) greed for money as the central threat to biodiversity. On the other hand, economic solutions that include ecological, economic and social aspects were not taken into account. Therefore, although pupils of this type tried to integrate economic issues, they mostly did not succeed in doing so. In contrast, the balanced type served as a positive example of how pupils succeed in integrating perspectives in accordance with the three pillars of sustainable development. That such integration is important is not only due to the fact that many national and international legislations explicitly demand education for sustainable development (e.g. CDLA, 2002; Deutscher Bundestag, 2005; UNCED, 1992; UNECE, 2005; UNESCO, 2005). Moreover, pupils who showed the subjective theory "loss 
of biodiversity due to social reasons" showed solidarity and understanding of people in resource dilemmas more easily.

\section{(Q 1.3) How do cognitive frameworks differ between Chilean and German pupils?}

The central research outcomes that address this question were the following:

(1) Chilean pupils mentioned numerous local plants and animals that are under threat, while all but one German pupil did not mention local examples at all.

(2) The balanced type being the group of pupils who integrated social aspects when talking about biodiversity loss and resource dilemmas, mainly consisted of German pupils.

(3) The local Chilean dilemma example, which was introduced during the interviews, was not identified by a group of five pupils, all of them Chilean.

The original motivation for including a Chilean sample in the study was the fact that Chile contains a biodiversity hotspot (Mittermeier et al., 2004). Furthermore, resource dilemmas are present in Chilean pupils' environment. It was therefore assumed that Chilean pupils would have a firsthand view of biodiversity and biodiversity loss. As a consequence, it would be easier for the Chilean interviewees to identify social aspects in a resource dilemma. However, the results did not support these expectations. On the one hand, Chilean pupils quoted numerous local examples of threatened species. On the other hand, the examples were not put into the context of resource dilemmas although the interview question explicitly had this focus (see appendix A). In support of the above, five Chilean pupils were not able to recognize the resource dilemma of the Chilean medical plant (Boldo) that was used as an example during the interview. Although the two dilemma examples that were presented during the interviews showed numerous parallels, these Chilean pupils did not see the problem associated with the local example at all. This means that the respective group of pupils did not even recognise the ecological aspects of the loss of Boldo.

One possible explanation for this phenomenon is the abundance of this medical plant at local markets. This observation might have misled pupils in that they could not believe that the plant is under threat, or could be in the future. If pupils see Boldo every day, it is possibly difficult to understand that such a plant might need protection, or, at least, careful patterns of utilisation. This finding is especially interesting as it indicates a perspective that is connected to the traps of resource dilemmas (Ernst, 1997). Although the interviewees did not actively 
participate in exploitation of natural resources, a local perspective of abundant natural resources can be an obstacle. Pupils apparently refer to what they currently see, without being able to judge consequences in the long run. Therefore, the consequences of overuse of natural resources are not realistically assessed.

There is more evidence generally for Chilean pupils' greater difficulties to recognise resource dilemmas. As reflected by the typology, only one Chilean pupil associated with the balanced type, being the group of pupils that considered social issues all throughout the interview. This means that, even those pupils of the Chilean sample who noted that there are problems associated with the local dilemma example, did not usually refer to the social facets of the problem. In most cases, economic problems, such as the difficulty to find an appropriate marketing strategy for the plant, or ecological problems, like the inability of the plant to be cultivated, were mentioned. In summary, only one Chilean pupil - the only Chilean associate of the balanced type - considered that people might depend on an income from selling the plant.

Within the German sample, pupils did not usually mention local examples of resource dilemmas or plants under threat (except for one example). In fact, resource dilemmas have a different dimension in Germany, as people usually do not depend on undomesticated or wild growing natural resources as a means of subsistence. However, numerous examples of threatened species exist (Ludwig \& Schnittler, 1996) that the pupils did not refer to. The German pupils within our qualitative sample rather applied international examples, often considering the difficult social and economic situations of those people who trade the respective plant or animal species. Therefore, for the German pupils interviewed, consideration of resource dilemmas was apparently easier. It is difficult to find an explanation for this phenomenon besides an already existing sensitivity towards questions of sustainable development amongst some German pupils. The fact that both dilemma examples were new to the pupils probably facilitated a transfer from social aspects of the first dilemma example, the Devil's Claw, to the second, Boldo. In contrast to the Chilean pupils, no direct experiences with abundant resources at local markets influenced the findings. Therefore, the similarity of the two examples might have been easier to note for the German pupils. 


\subsection{Chilean and German pupils' commitment to protect biodiversity (research focus II)}

For the quantitative part of the study (research focus II), an overall hypothesis and one research question were formulated. Relevant research findings were presented in chapters eight and nine. As in the previous chapter (10.1), central research findings will be briefly sketched. Then, a general discussion of the research findings follows.

(H 2.1) Chilean and German pupils differ in their personal norms and commitments to protect biodiversity through three different behavioural commitments namely, activism, non activist, public-sphere behaviour and private-sphere behaviour.

The central results that addressed this hypothesis were the following:

(1) Chilean pupils showed higher personal norms,

(2) higher commitment to protect biodiversity through activism,

(3) through non activist, public-sphere behaviour and

(4) through private-sphere behaviour than German pupils.

Our results support hypothesis one (H 2.1). Furthermore, besides the hypothesised differences between the two samples, Chilean pupils scored higher on all the scales that were included in the hypothesis. The differences showed medium or high effects.

Due to the presence of biodiversity loss as an observable problem, the respondents may feel more affected by the consequences of biodiversity loss than the German pupils. As a consequence, Chilean pupils might feel a stronger wish to contribute to the solution of the problem. This interpretation is supported by the fact that Chilean pupils also scored higher on an awareness of egoistic consequences. For example, Chilean pupils might observe the serious decline of natural forests that are cleared in favour of more profitable pine tree plantations (Figueroa \& Simonetti, 2003) and deduce consequences affecting their own lives. This explanation is likely if we reflect the contexts of items that were used to measure awareness of egoistic consequences. For example, the items included statements about places of high biodiversity that serve as spaces for recreation and enjoyment. Therefore, Chilean pupils might indeed feel a stronger personal involvement. According to the underlying VBN theory (Stern et al., 1995a; Stern et al., 1999; Stern, 2000), such egoistic awareness of consequences can lead to higher personal norms and, in turn, to higher commitments to pro- 
environmental behaviour. Therefore, within the Chilean sample, the personal consternation reflected by awareness of egoistic consequences was higher than in the German sample (and also showed an influence on commitments to protect biodiversity in the Chilean sample, see chapter nine).

However, despite numerous serious environmental problems (among them biodiversity loss), environmental protection is only slowly gaining public interest in most Latin American countries, including Chile (Rieckmann, 2004). At the present time, many behavioural options that are already part of many peoples' everyday routines in Germany do not exist in Chile. Therefore, we should consider that Chilean pupils did not see the actual personal costs of the behaviour that they committed themselves to. As a consequence, Chilean pupils might show high commitment to behaviours that they cannot judge realistically in time and effort.

In comparison, German pupils' commitment to protect biodiversity was clearly lower. In accordance with the interpretation that Chilean pupils' higher commitments to protect biodiversity are due to their personal consternation, a lower personal consternation could have led to the German pupils' lower commitments to protect biodiversity. Additionally, German pupils might judge time and effort to be invested in pro-environmental behaviours more realistically. In support of our results, a generally low environmental concern has been described in recent empirical studies in Germany. For example, Hurrelmann et al. (2006) reported that environmental problems are subordinate on German young peoples' priority list, in favour of problems such as unemployment or the well-being of ones own family. Another explanation of German pupils' lower commitments to protect biodiversity is the effect of fatigue towards pro-environmental behaviour. Environmental issues might come after the benefits of other interests, especially for adolescents. Our finding that behavioural commitment towards activism was especially low is also supported throughout literature. A recent study with German adolescents found that young people prefer private activities, such as consumer choices, to activist behaviour, when commiting themselves to protecting the environment (Greenpeace, 2005).

(Q 2.1) Which predictors are relevant to explain Chilean and German pupils' commitments to protect biodiversity? How well do the regression models explain the three different commitments to protect biodiversity in the two samples?

The following predictors showed to be relevant in a regression model with selected predictors covering values, beliefs, and personal norms. Predictors were selected as the 
results of a first regression model, in which values, beliefs, and norms were regressed separately on each type of behavioural commitment.

(1) Personal norms proved to be a powerful predictor for commitment to activism, non activist, public-sphere behaviour and private-sphere behaviour in both samples.

(2) Within the Chilean sample, the following set of relevant predictors emerged:

a. For commitment to protect biodiversity through activism, the value of security and ascription of responsibility had a positive influence, besides personal norms. For activism, the explained variance was $\boldsymbol{a d j} . \boldsymbol{R}^{2}=\mathbf{0 . 4 5}$.

b. For non activist, public-sphere behaviour, the value of tradition was the only positive predictor besides personal norms. Adj. $\boldsymbol{R}^{2}$ was 0.31 .

c. For commitment to private-sphere behaviour, the NEP and perceived ability to reduce threats had a positive influence besides personal norms, which lead to an $\boldsymbol{a d j} . \boldsymbol{R}^{2}$ of $\mathbf{0 . 3 8}$.

(3) Within the German sample, the three types of behavioural commitment were explained by the following predictor sets:

a. The value universalism showed high relevance for all behavioural types within the German sample.

b. For commitment to activism, security had a negative influence and ascription of responsibility had a positive influence, besides universalism and personal norms, which lead to an $\boldsymbol{a d j} . \boldsymbol{R}^{2}$ of 0.45 .

c. To explain non activist, public- sphere behaviour, the NEP proved to be relevant apart from universalism and personal norms with an adj. $\boldsymbol{R}^{2}$ of 0.42 .

d. Five predictors explained commitment to private-sphere behaviour. Universalism had a positive influence, as did self-direction. In contrast, stimulation predicted commitment to private sphere behaviour negatively. Apart from personal norms, perceived ability to reduce threats to biodiversity was a relevant predictor. The amount of explained variance was adj. $R^{2}=0.53$.

Regarding the role of values, a generally weak influence emerged within the Chilean sample. Therefore, values in the Chilean sample might rather influence beliefs, which would then, in turn, influence behavioural commitments directly or indirectly. For example, for non activist, public-sphere behaviour, the value of tradition proved to be a positive predictor within the 
Chilean sample. This finding is interesting as tradition has been described as a negative predictor for pro-environmental behaviour in literature, which our data contradicts (Stern et al., 1995a). There are possibly traditional values that Chilean pupils draw upon that foster the protection of nature. Besides tradition, security showed a positive influence on the commitment for activism. This means that those who appreciate security would more likely commit activist behaviour to protect biodiversity. This result suggests that Chilean pupils possibly note a risk of security caused by biodiversity loss, which then motivates a commitment to activism (note that security influenced activism negatively in the German sample). The afore-mentioned relevance of awareness of egoistic consequences suggests the same: within the Chilean sample, with beliefs as exclusive predictors in analysis I, a personal consternation seemed to be more important to explain behavioural commitments than in the German sample.

In contrast, in the German sample, the value "universalism" proved to be a strong predictor for all types of behavioural commitment. The importance of the value universalism is understandable as it embodies aspects such as justice, respect and appreciation of nature. Overall, no matter to which type of behaviour German pupils commit themselves to, universalism plays a central role. In a study on the influence of Schwartz values on proenvironmental behaviour, Guiterrez Karp (1996) reported that the value cluster "selftranscendence", which is a cluster of the two Schwartz values of universalism and benevolence, positively influenced pro-environmental behaviour (cf. also Stern et al., 1995a). A possible explanation of the negative influence of the value of security on activism in the German sample is that pupils who generally appreciate security in their environment, would probably not participate in sometimes dangerous activities, such as tagging a protest banner on the outside of a company. Moreover, a positive role of the value of self-direction in order to predict private-sphere behaviour occurred in the German sample. This finding suggests that the protection of biodiversity in the private sphere might serve as an expression for individualism. For example, pupils may acknowledge the possibility to make their own choices in the private context. In contrast, the negative influence of stimulation on privatesphere behaviour is more difficult to explain. Stimulation is a value that expresses an affinity towards a stimulating and varied life. Possibly, some pupils think of private sphere behaviour to protect nature to be bourgeois. This could be due to a high level of routine that some German pupils might experience in pro-environmental private-sphere behaviour (such as recycling).

With regards to beliefs, similar predictors were relevant in both samples to explain the three different kinds of behavioural commitments. Therefore, beliefs will in the following be discussed together for both samples. 
For activism, an ascription of responsibility had a positive influence in both samples. It suggests itself that those who feel responsible for the protection of biodiversity will also show a higher commitment to do so. Apparently, a perceived responsibility is particularly important to commit activism as this kind of behavioural commitment probably bears the highest personal costs for the pupils. As a consequence, only those who feel highly responsible would get involved in this kind of pro-environmental behaviour that can be provoking in public, and might also have negative consequences for an individual.

For a commitment to non activist, public-sphere behaviour, no predictors that belong to the "beliefs" block were relevant in the Chilean sample. In the German sample, the NEP was relevant for this type of behavioural commitment. That a general conviction of humans and nature being closely related as expressed by the NEP, is conductive to a commitment to protect nature has been reported before (cf. Dunlap \& van Liere, 1978; Dunlap et al., 2000; Schultz \& Zelezny, 1998; Stern et al., 1995b) and can, thus, be supported by our data.

For commitment to private-sphere behaviour, a perceived ability to reduce threats showed to be a relevant predictor in both samples. This result suggests that commitments to privatesphere behaviour, which are not related to public activity, depend particularly on the pupils' conviction that the respective behaviour is a real contribution to biodiversity protection. Other than public-sphere behaviours, private sphere behaviours are more likely to be decisions that influence behaviours on the long run. For example, changing the trade mark of cosmetics due to unsustainable production patterns is a decision that has to be retained, and not only shown once (in contrast to, for example, signing a petition as non activist, publicsphere behaviour). In this regard, the belief that such behaviour is actually efficient, is obviously important. Besides such a perceived ability to reduce threats, the NEP showed to be relevant in the Chilean sample for private-sphere behaviour. Here again, a perspective of humans depending on nature shows a positive influence on a commitment to proenvironmental behaviour.

The assumption of personal norms as a central predictor for altruistic behaviour was based on Schwartz' Norm-Activation Model (Schwartz, 1977; Schwartz \& Howard, 1981). In turn, as the VBN theory is based on the Norm-Activation Model, personal norms are assumed to have a strong influence on pro-environmental behaviour. The results of this study supports the assumed importance of personal norms. According to Schwartz (1977), personal norms are closely connected to a person's self-concept (cf. chapter 3.2). In the case of Chilean and German pupils, a strong self-concept with regards to behaviour to protect biodiversity strongly influenced all three kinds of behavioural commitments. However, due to the close relation between personal norms and behavioural commitments, it is possible that the same, 
or similar, underlying factors influenced the two constructs. Thus, in a future perspective, it would be interesting to investigate the predictors for personal norms.

Combined, we can summarize that the VBN theory served as a suitable theoretical background to explain Chilean and German young peoples' commitment to protect biodiversity as high effects of VBN constructs to explain all three kinds of behavioural commitments emerged. According to the percentage of explained variance, the VBN Theory seemed to be slightly more suitable to explain commitment to protect biodiversity in the German sample. Regarding the different kinds of behavioural commitments, Stern et al. (1999) reported the lowest percentage of explained variance for commitment to participate in a demonstration (reflecting activist behaviour) and concluded that the VBN theory might be less suitable to explain commitment to activism when compared to non activist, publicsphere behaviour. However, our results showed that predictors that were deduced from the VBN theory were suitable to explain activism in both samples. Actually, in the Chilean sample, the highest percentage of explained variance was found for commitment to protect biodiversity through activism. The comparably lower percentage of explained variance for non activist, public-sphere behaviour, especially in the Chilean sample, could be due to the items that were used in our questionnaire in order to measure this kind of behavioural commitment. Signing a petition for the protection of endangered species, or writing a letter to the government with the aim of pointing out the necessary protection of endangered species, might be motivated by other, additional factors that we did not assess. However, although lower in comparison to the other kinds of behavioural commitments, the percentage of explained variance for non activist, public-sphere behaviour was still satisfying in both samples.

\subsection{Critical discussion}

The results of research focus I showed that an interdisciplinary perspective on the biodiversity topic was challenging for most pupils from both countries. However, we have to assume that the interview was the first occasion for most respondents to think and speak about biodiversity and biodiversity loss in detail. Presumably, in the school context, some pupils would develop a deeper and less naïve understanding of the topic when it would be treated i) in detail and ii) under careful consideration of possibly inhibiting cognitive frameworks. In this respect, the results of the qualitative study provide valuable hints on how such a deeper understanding can be achieved. 
Another critical point of the qualitative study is the small sample size and, therefore, restrictions in generalisations. On the one hand, the decision to conduct in-depth interviews and data analysis took its toll with respect to sample sizes. On the other hand, due to a manageable number of interviews, a deeper analysis could be conducted which was important to be able to identify cognitive frameworks. With regards to the sample, generalisations are not only problematic due to the small number of interviewees. Subjective theories derive from different contexts and life experiences (e.g. Piaget, 1974; Posner et al., 1982) and are, therefore, highly individual. However, general societal notions can also strongly influence subjective theories (as demonstrated through the brain-twister about the woman surgeon). Therefore, although investigated with individuals, the investigation of subjective theories can still provide useful information on cognitive frameworks whose existence can be assumed beyond the individual.

The most prominent subjective theory that formed the basis of the most prominent type was "loss of biodiversity due to ecological reasons". A possible explanation for such a widespread disciplinary orientation could be that pupils thought that a disciplinary way of thinking is more sophisticated. Although we conducted the interviews divorced from science classes, the term biodiversity might already have influenced pupils to think in a disciplinary way. Moreover, the fact that the interviews were conducted for a scientific investigation (which was communicated to each pupil prior to the interview) might have fostered imagined socially desired answers. Therefore, pupils might have a better understanding of social issues than they actually showed during the interviews. However, the finding that most pupils still did not succeed in integrating social and economic aspects after respective examples were provided, cannot exclusively be explained by a socially desired disciplinary orientation.

The subjective theories that were identified were broad and general in that they either permitted or inhibited the inclusion of social aspects and, hence, a balanced consideration of biodiversity loss. In addition to these general subjective theories, one must assume that within individuals of the two samples, more subjective theories existed although they were not identified within our study. Nevertheless, the theories that were discovered and which then served as the basis for the typology, seemed to be the most relevant within the samples with respect to the perception of biodiversity and biodiversity loss. Although subjective theories and the typology emerged from a small sample and qualitative analyses, the results showed that a substantial number of pupils had problems with the understanding of social aspects in resource dilemmas and, thus, biodiversity loss.

Regarding research focus II; again, some critical points have to be mentioned. Firstly, we should bear in mind that the samples, although clearly larger than those of the qualitative study, were still not representative, neither for the Chilean and German society, nor for 
pupils of the two countries. Moreover, pupils were all enrolled in secondary education and therefore had access to higher education. As a consequence, the participants should not be regarded as a typical cross-section of pupils within both countries. In addition, the sample was a cluster sample as questionnaires were distributed as class sets. Cluster samples can possibly lead to an increase of the $\alpha$-error in quantitative studies (Rost, 2005). However, due to organisational restrictions, random samples on the basis of individual pupils could not be realised.

Secondly, it is arguable whether personal norms superceded weaker, but still important, predictors, especially in the Chilean sample, due to their proximate position to behavioural commitments in the VBN theory. We tried to address this problem by conducting regressions separately for values, beliefs, and norms in our initial regression models on each type of behavioural commitment (analysis I). However, it would be interesting to use personal norms as dependent variable in order to learn more about factors that influence this important predictor.

A third critical point of the quantitative study is a potential cultural bias reflected by the positive response tendency of the Chilean sample. However, as Chilean scores were lower or not significantly higher on some constructs, the more positive responses presumably reflected an actual higher concern and consequent commitment of Chilean pupils. Therefore, although a possible cultural response bias can not completely be dispelled, differences are interpreted to be meaningful due to the fact that Chilean values were not constantly higher for the Chilean sample.

\section{Educational Implications and Concluding Remarks}

According to a constructivist perspective on the learner, cognitive frameworks are important structuring elements to process new information (Caravita \& Halldén, 1994; Carey, 1985; Driver \& Easley, 1978; Piaget, 1974; Posner et al. 1982). Therefore, for teaching processes, it is helpful to consider pupils' prior cognitive frameworks. In that way, teachers can either build upon existing ideas or actively address frameworks that are potentially opposed to new teaching contents (Duit \& Treagust, 2003; Posner et al., 1982; Vosniadou, 1992, 1994). However, teachers do usually not have the chance to investigate pupils' cognitive frameworks in detail. Thus, for teaching practices, the above-condensed research findings can contribute to an understanding of prominent cognitive frameworks. 
With respect to pupils' associations with the terms "biodiversity" and "biological diversity", concentration on the diversity of species was prominent. However, many pupils also included the ecosystems level when spontaneously describing biodiversity. In this regard, factual information on a proper definition of biodiversity and biological diversity might already be sufficient to counteract cognitive frameworks that are too restricted in that they focus on species diversity.

In the case of values of biodiversity, educational interventions can build upon the prominent concept that biodiversity is useful for humans, in that natural resources are important for the production of commodities. However, the concept should also be actively broadened in order to point out the intrinsic value of biodiversity, or, at least the discussion surrounding the dispute of whether there is such absolute value for nature itself (Krebs, 1997, Naess, 1997). In this regard, the much less prominent concept of genetic diversity, as insurance for survival under changing conditions, might be an interesting starting point that teaching interventions can build upon.

The concept of "lack of space" served to explain low biodiversity in cities and urban areas and was problematic in two regards: Firstly, it might prevent pupils from seeing that cities are also important ecosystems (e.g. Kahn, 2006). Secondly, the concept did not acknowledge habitat destruction in developing countries (cf. Hilty et al., 2006). However, the concept of "lack of space" is a valuable starting point if it is broadened to the global problem of habitat destruction. Moreover, through a teaching sequence about urban ecology, cognitive conflicts can be provoked where pupils have to recognize species and ecosystems diversity in urban areas.

The second concept regarding the distribution of biodiversity that emerged from the interview data was one of low biodiversity due to climatic extremes. However, although inappropriate at first sight, teaching contents could also build upon this concept and broaden it. In order to achieve this goal, the core principle to integrate in biodiversity education is evolution. If, for example, biodiversity hotspots are discussed under an evolutionary perspective, cognitive conflicts may arise in the pupil in that he or she is confronted with the fact that difficult ecological conditions predominate at most hotspots. Moreover, pupils will learn that endemic plants are often a result of a higher pressure to adopt to difficult ecological circumstances. One example are the numerous phytosymbioses in coral reefs that are evolutionary adaptations to extreme nutrient-deficiency (Stanley, 2006). Only by expatiating these interrelations can pupils be prepared for the emerging public discussion on climate change. In this regard, it is important to enable pupils to distinguish between extreme environmental conditions that during evolution led to high levels of biodiversity, and human- 
caused, rapidly emerging extremes that disturb delicate ecological interrelations and, as a consequence, cause biodiversity loss.

A central result of the interview study was that integration of ecological, economic, and social information - the basis for a proper understanding of resource dilemmas in the sense of sustainable development - was a serious challenge for many pupils. Overall, the most important consequence for teaching practice is to acknowledge that it is not sufficient to purely provide information about social and economic drivers for biodiversity loss. Those pupils, whose subjective theories allow inclusion of such information, might succeed in an adequate consideration of the three spheres of sustainable development. However, for those pupils who tend to focus on ecological or economic aspects, a pure presentation of information might not be sufficient. As a consequence, pupils stay with their prior frameworks.

Moreover, the results suggested that a strong concentration on ecological reasons for biodiversity loss might lead pupils to apply inappropriate ecological arguments whenever "accurate" ecological arguments fail to explain biodiversity loss. Therefore, sophisticated teaching concepts are necessary that can foster conceptual growth in order to integrate all three pillars of sustainable development, including the socio-economic situation of stakeholders of natural resources. To achieve this goal, innovative teaching approaches are necessary. The research framework of "didactical reconstruction" (Gropengießer, 2003; Kattmann et al., 1997), suggests particular learning tasks and experiments that may provoke cognitive conflicts in pupils. In the context of biodiversity, approaches such as role-plays are imaginable, in that pupils either take on the role of people who depend on natural resources, or of advocates who defend the rights of stakeholders in an exemplary resource dilemma. Therefore, biodiversity education would impart real-world approaches that reflect realistic challenges that biodiversity conservation faces. Moreover, approaches such as "science education for citizenship" (Ratcliffe \& Grace, 2003) and the socio-ecological approach to environmental education (Kyburz-Graber, 2006) provide ideas and guidelines that could also be applied to the context of biodiversity.

Another central consequence for teaching processes, that can be drawn from the results of the Chilean sample, is that one must not assume that a better understanding of resource dilemmas is authentic just because it happens on the learner's doorstep. However, the results show that learners construct knowledge from their life experiences, even when this can be misleading, such as in the case of the Chilean pupils interviewed. Therefore, in the Chilean context, teaching interventions that foster a change in perspectives and empathy towards people in resource dilemmas are important. 
In contrast, for the German pupils of our qualitative sample, it was apparently easier to recognise resource dilemmas. However, German pupils mentioned few local examples of threatened species. Therefore, in Germany, biodiversity education should impart a local perspective on biodiversity loss even if the global aspects are just as important. In addition, such a local perspective can also be helpful for the enhancement of acknowledgement of biodiversity (Lindemann-Matthies, 2002b, 2004). With regard to resource dilemmas, it is certainly difficult to find local examples which are comparable to resource dilemmas in developing countries. However, at least historical examples exist within the European context, such as the dramatic deforestation in the $18^{\text {th }}$ century (Johann, 2002).

What educational implications can be drawn from the findings of the quantitative study? Firstly, we should bear in mind that the quantitative part reflects basic research. Therefore, it is difficult to draw direct educational implications from some of the results. However, our data shows that, for biodiversity education, we have to assume different influencing psychological factors for Chilean and German pupils.

The central motif for Chilean pupils' commitment to protect biodiversity seems to be their personal concern and consternation. In order to foster commitment to protect biodiversity, it would be helpful to point out consequences of biodiversity loss on a local level. At the same time, educators should bear in mind that biodiversity loss is a global phenomenon. Even when consequences can be noticed on a local level, global interrelations are often important drivers for biodiversity loss (consider the "spatial trap" of resource dilemmas as presented in the introduction). Moreover, if pupils are regarded as the possible decision makers of tomorrow, they must be able to understand global interrelations in order to be able to make or support - the right decisions. In addition, by developing realistic behavioural perspectives, especially for private-sphere behaviour, a perceived ability to reduce threats should also be encouraged. However, due to the complexity of the biodiversity topic, including the global dimension of the phenomenon of biodiversity loss, it might be difficult for pupils to develop realistic - and effective! - behavioural perspectives. Biodiversity education can contribute in this respect by shedding light upon the role that individuals play. For example, Brower and Leon (1999) and Davidson \& Hatt (2005) suggest simple and yet effective changes in everyday consumption patterns that foster the protection of natural resources in the global perspective of sustainable development. Such practical guides could actively support a perceived ability to reduce threats to biodiversity, and, therefore, commitment to protect biodiversity. Such educational measures can, once pupils notice what they can contribute, at the same time contribute to the enhancement of ascription of responsibility to the self, which, above all, plays a role for the commitment to activism. 
For the German sample, we should bear in mind that universalism as a basic human value cannot be "taught" as such. Values are stable structures in the human psyche and are difficult to change (Schwartz, 1992, 1994). However, biodiversity education could build upon the value of universalism by focusing on aspects such as justice and solidarity. These are important elements of the biodiversity topic. Above all, the use and overuse of natural resources, which is a serious threat to biodiversity, touch ethical questions like rights over natural resources and benefit sharing. Besides the value of universalism, ascription of responsibility proved to be important in the German sample, especially for activism (moreover, in an analysis with exclusively beliefs as predictors, ascription of responsibility was relevant to all behavioural commitments in both samples, apart from private-sphere behaviour in the Chilean sample). It is, therefore, important for both Chilean and German pupils to foster their awareness of their own portion within the complicated interrelations that put biodiversity at risk. As shown by our data, pupils from the industrial country Germany, seem to have greater difficulties than pupils living close to a hotspot to see consequences of biodiversity loss in their own lives. In support of these findings, the qualitative study with Chilean and German pupils could demonstrate these difficulties exemplarily to get involved on both consternation and realistic perspectives to actually do something about the problem.

The research that was conducted and reported in this dissertation aimed at contributing to sustainable development, and, especially, to the protection of biodiversity. The current biodiversity crisis is virtually completely due to human activity (McKee, 2003; Sinclair, 2000; Wilson, 2001). In order to sustain the living environment for future generations, humanity is now in charge of the limitation of damage through biodiversity protection (CBD, 1992; UNCED, 1992; WCED, 1987). In order to achieve biodiversity conservation, involving people is a "must" and biodiversity education is among the most important vehicles to achieve such an involvement (CBD, 1992; UNCED, 1992; WCED, 1987).

Important learning preconditions must be taken into account in order to design effective educational measures. This work addressed learning preconditions under two foci by investigating i) pupils' cognitive frameworks and ii) fostering and inhibiting factors on commitment to protect biodiversity. In this respect, the central research findings provide valuable starting points for biodiversity education.

However, much work remains to be done. Besides the expansion of the presented work, the results have to be put into practice. In this process, the outcomes of the project of "learning 
prerequisites for biodiversity" will hopefully contribute to an evidence-based biodiversity education to sustain biodiversity on both, local and global levels. 


\section{References}

Adams, W. M. (2001). Green development. Environment and sustainability in the Third World (2nd edition). New York: Routledge.

Allen, T., Tainter, J. A., \& Hoekstra, T. (1999). Supply-side sustainability. Systems Research and Behavioral Science, 16, 403-427.

Allen, T., Tainter, J. A., \& Hoekstra, T. (2003). Supply-side sustainability. New York: Columbia University Press.

Balmford, A., Clegg, G., Coulsen, T., \& Taylor, J. (2002). Why conservationists heed Pokémon. Science, 295, 2367.

Bamberg, S., \& Schmidt, P. (2003). Incentives, Morality, or Habit? Predicting Students' Car Use for University Routes With the Models of Ajzen, Schwartz, and Triandis. Environment and Behavior, 35, 264-285.

Bamberg, S., \& Möser, G. (2007). Twenty years after Hines, Hungerford, and Tomera: A new meta-analysis of psycho-social determinants of pro-environmental behaviour. Journal of Environmental Psychology, 27, 14-25.

Barkmann, J., \& Bögeholz, S. (2003). Kompetent gestalten, wenn es komplexer wird - eine kurze Einführung in die ökologische Bewertungs- und Urteilskompetenz. Zeitschrift "21", 3, 49-52.

Bayrhuber, H., Bögeholz, S., Elster, D., Hößle, C., Lücken, M., Mayer, J., Nerdel, C., Neuhaus, B., Prechtl, H., \& Sandmann, A. (2007). Biologie im Kontext (bik) - Ein

Programm zur Kompetenzförderung durch Kontextorientierung im Biologieunterricht und zur Unterstützung von Lehrerprofessionalisierung. MNU, 304-313.

Bebbington, A. (2005). The ability of A-level students to name plants. Journal of Biological Education, 39, 62-67.

Bechtel, R. B., Corral Verdugo, V., \& de Queiroz Pinhero, J. (1999). Environmental belief systems. Journal of Cross-Cultural Psychology, 30, 122-128.

Bell, A. C., Russell, C. L., \& Plotkin, R. (1998). Environmental learning and the study of extinction. Journal of Environmental Education, 29, 4-9. 
Berenguer, J. (2007). The effect of empathy in proenvironmental attitudes and behaviors. Environment and Behavior, 39, 269-283.

Berndt, K.-P. (2000). Interdisziplinäres multimediales Lehr- und Lernprojekt "Biodiversität in Großökosystemen". Brandenburgische Umwelt Berichte, 8, 12-20.

Black, J. S., Stern, P. C., \& Elsworth, J. T. (1985). Personal and contextual influences on household energy adaptations. Journal of Applied Psychology, 70, 3-21.

Blaikie, N. W. H. (1992). The nature and origins of ecological world views: An Australian study. Social Science Quarterly, 73, 144-165.

Bögeholz, S. (1999). Qualitäten primärer Naturerfahrung und ihr Zusammenhang mit Umweltwissen und Umwelthandeln. Opladen: Leske und Budrich.

Bögeholz, S. (2001). Möglichkeiten und Grenzen von „empirischen“ Naturerfahrungstypen in der Umweltbildung. In G. de Haan, E. D. Lantermann, V. Linneweber \& F. Reusswig (Eds.), Typenbildung in der sozialwissenschaftlichen Umweltforschung (pp. 243-259). Opladen: Leske und Budrich.

Bögeholz, S., \& Barkmann, J. (1999). Kompetenzerwerb für Umwelthandeln: psychologische und pädagogische Überlegungen. Die Deutsche Schule, 4, 93-101.

Bögeholz, S., Bittner, A., \& Knolle, F. (2006). Der Nationalpark als Bildungsort. Vom Naturerleben zur Bildung für eine Nachhaltige Entwicklung. Gaia, 15, 135-143.

Bogner, F. X., \& Wiseman, M. (2002). Environmental perception: Factor profiles of extreme groups. European Psychologist, 7, 225-237.

Bortz, J. (1993). Statistik für Sozialwissenschaftler (Band 4). Berlin, Heidelberg, New York u. a.: Springer.

Bright, A. D., \& Tarrant, M. A. (2002). Effect of environment-based coursework on the nature of attitudes toward the endangered species act. The Journal of Environmental Education, 33, 10-19.

Brower, M., \& Leon, W. (1999). The consumer's guide to effective environmental choices: practical advice from the Union of Concerned Scientists. New York: Random House.

Caravita, S., \& Halldén, O. (1994). Re-framing the problem of conceptual change. Learning and Instruction, 4, 89-111.

Carey, S. (1985). Conceptual change in childhood. Cambridge: MIT Press.

CBD - Convention on Biological Diversity (1992). Rio de Janeiro: UNCED United Nations Conference on Environment and Development. 
Cincotta, R. P., Wisnewski, J., \& Engelman, R. (2000). Human population in the biodiversity hotspots. Nature, 404, 990-992.

CDLA - Consejo de las Américas Chile (2002). Participación ciudadana y desarrollo sustantable. Bases de licitación: Proyecto gestión ambiental local participativa y cooperativa para el Desarrollo sustentable. Santiago de Chile: CDLA.

Dann, H.-D. (1994). Pädagogisches Verstehen: Subjektive Theorien und erfolgreiches Handeln von Lehrkräften. In K. Reusser \& M. Reusser-Weyeneth (Eds.), Verstehen. Psychologischer Prozeß und didaktische Aufgabe. Göttingen, Toronto, Seattle: Huber.

Davidson, D. J., Hatt, K. C., \& the Northern Critical Scholars Collective (2005). Consuming sustainablility. Halifax: Fernwood Publishing.

Delaney, H., \& Vargha. A. (2000, April 24-28). The effect of nonnormality on Student's two sample t-test. New Orleans: paper presented at the American Educational Research Association.

Deutscher Bundestag (2005). Bericht der Bundesregierung zur Bildung für eine nachhaltige Entwicklung für den Zeitraum 2002-2005. Berlin.

Devall, B. (2001). The deep, long-range ecology movement. Ethics and the Environment, 6, 18-41.

Devitt, M. (1984). Realism and truth. Oxford: Blackwell.

Dietz, T., Ostrom, E., \& Stern, P. C. (2003). The struggle to govern the commons. Science, 302, 1907-1912.

Dreyfus, A., Wals, A. E. J., \& van Weelie, D. (1999). Biodiversity as a postmodern theme for environmental education. Canadian Journal of Environmental Education, 4, 155175.

Driver, R. (1989). 'Changing conceptions'. In P. Adey, J. Bliss, J. Head \& M Shayer (Eds.), Adolescent development and school science (pp. 79-99). London: Falmer Press.

Driver, R., \& Erickson, G. (1983). Theories in action: some theoretical and empirical issues in the study of students' conceptual frameworks in science. Studies in Science Education, 10, 37-60.

Driver, R., \& Easley, J. (1978). Pupils and paradigms: a review of literature related to concept development in adolescent science students. Studies in Science Education, 5, 61-84. 
Duit, R., \& Treagust, D. (2003). Conceptual change: a powerful framework for improving science teaching and learning. International Journal of Science Education, 25, 671688.

Dunlap, R. E. (2002). Environmental sociology. In R. B. Bechtel \& A. Churchman (Eds.), Handbook of Environmental Psychology. New York: John Wiley \& Sons.

Dunlap, R. E., \& van Liere, K. D. (1978). The New Environmental Paradigm. The Journal of Environmental Education, 9, 10-19.

Dunlap, R. E., van Liere, K. D., Mertig, A. G., \& Jones, R. E. (2000). Measuring endorsement of the New Ecological Paradigm: a revised NEP scale. Journal of Social Issues, 56, 425-442.

Eagan, P., Cook, T., \& Joeres, E. (2002). Teaching the importance of culture and interdisciplinary education for sustainable development. International Journal of Sustainability in Higher Education, 3, 48-66.

Ernst, A. (1997). Ökologisch-Soziale Dilemmata. Heidelberg: Psychologie Verlags Union.

Erwin, T. L. (1982). Tropical forests: the richness in Coleoptera and other arthropod species. Coleopterists Bulletin, 36, 74-75.

Field, A. (2005). Discovering statistics using SPSS. London, Thousand Oaks, New Dehli: Sage Publications.

Fien, J. (2002). Advancing sustainability in higher education: issues and opportunities for research. Higher Education Policy, 15, 143-152.

Figueroa, E. B., \& Simonetti, J. A. (2003). Globalización y biodiversidad: Oportunidades y desafíos para la sociedad chilena. Santiago de Chile: Editorial Universitaria.

Flenley, J., \& Bush, M. (2006). Tropical rainforest responses to climate change. Berlin: Springer.

Flint, W., McCarter, W., \& Bonniwell, T. (2000). Interdisciplinary education in sustainability: links in secondary and higher education - the Northhampton Legacy Program. International Journal of Sustainability in Higher Education, 1, 191-202.

Flitner, M. (1999). Das Öl, das Meer und die "Tragödie der Gemeingüter". In C. Görg, C. Hertler, E. Schramm \& M. Weingarten (Eds.), Zugänge zur Biodiversität (pp. 53-70). Marburg: Metropolis.

Gardner, G. T., \& Stern, P. C. (1996). Environmental problems and human behavior. Boston, London, Toronto u. a.: Allyn \& Bacon. 
Gayford, C. (2000). Biodiversity education: a teacher's perspective. Environmental Education Research, 6, 347-361.

Georghiades, P. (2000). Beyond conceptual change learning in science education: focusing on transfer, durability and metacognition. Educational Research, 42, 119-139.

Gerstenmaier, J., \& Mandl, H. (1995). Wissenserwerb unter konstruktivistischer Perspektive. Zeitschrift für Pädagogik, 41, 867-888.

Glaser, B. G. \& Strauss, A. L. (1998). Grounded Theory: Strategien qualitativer Forschung. Bern: Huber.

von Glasersfeld, E. (2005). Radikaler Konstruktivismus: Ideen, Ergebnisse, Probleme. Frankfurt am Main: Suhrkamp.

Goody, J. (2001). Competencies and education: Contextual diversity. In S. D. Rychen \& L. H. Salganik (Eds.), Defining and selecting key competencies (pp. 175-189). Göttingen: Hogrefe \& Huber.

Grace, M., \& Ratcliffe, M. (2002). The science and values that young people draw upon to make decisions about biological conservation issues. International Journal of Science Education, 24, 1157-1169.

Greenpeace (2005). Jugend und Umwelt. Hamburg: Greenpeace \& Emnid.

Grob, A. (1991). Meinung-Verhalten-Umwelt: Ein psychologisches Ursachennetz-Modell umweltgerechten Verhaltens. Bern, Berlin, Frankfurt: Peter Lang.

Groeben, N., Wahl, D., Schlee, J., \& Scheele, B. (1988). Das Forschungsprogramm Subjektive Theorien: eine Einführung in die Psychologie des reflexiven Subjekts. Tübingen: Francke.

Gropengießer, H. (2001). Didaktische Rekonstruktion des Sehens. Oldenburg: Didaktisches Zentrum der Universität Oldenburg.

Gropengießer, H. (2003). Wie man Vorstellungen der Lerner verstehen kann. Lebenswelten, Denkwelten, Sprechwelten. Oldenburg: Didaktisches Zentrum Oldenburg.

Guagnano, G. A. (1995). Locus of control, altruism and agentic disposition. Population and Environment, 17, 63-77.

Guagnano, G. A., Dietz, T., \& Stern, P. C. (1994). Willingness to pay for public goods: a test of the contribution model. Psycholgical Science, 5, 411-415.

Gutierrez Karp, D. (1996). Values and their effect on pro-environmental behavior. Environment and Behavior, 28, 111-133. 
Hallock, P., \& Schlager, W. (1986). Nutrient excess and the demise of coral reefs and carbonate platforms. Palaios, 1, 389-398.

Hardin, G. (1968). The tragedy of the commons. Science, 162, 1243-1248.

Helmers, B. (2004). Biologische Vielfalt in den Köpfen von Schülerinnen und Schülern: eine qualitative Untersuchung $\mathrm{zu}$ Schülervorstellungen von Biodiversität, damit verbundenen Werthaltungen und Gefährdungsfaktoren. Unpublished master's thesis, Göttingen University, Germany.

Helmers, B., Menzel, S., \& Bögeholz, S. (2004). Interviewleitfaden zu Schülervorstellungen zu biologischer Vielfalt. Unpublished interview guide, Göttingen University, Germany.

Herremanns, I., \& Reid, R. E. (2002). Developing awareness of the sustainability concept. The Journal of Enviromental Education, 34, 16-20.

Hesse, A. (2006). Big Six - die sechs wichtigsten, globalen Herausforderungen für Sustainable Development im 21. Jahrhundert. Unpublished doctoral dissertation, Erfurt University, Germany.

Hilty, J. A., Lidicker, W. Z., \& Merenlender, A. M. (2006). Corridor ecology. The science and practice of linking landscapes for biodiversity conservation. Washington D.C.: Island Press.

Hopper, J. R., \& Nielsen, J. M. (1991). Recycling as altruistic behavior: normative and behavioral strategies to expand participation in a community recycling program. Environment and Behavior, 23, 195-220.

Hunecke, M., Matthies, E., Blöbaum, A., \& Höger, R. (1999). Die Umsetzung einer persönlichen Norm in umweltverantwortliches Handeln. Umweltpsychologie, 3, 1022.

Hurrelmann, K., Albert, M., \& TNS Infratest Sozialforschung (2006). Jugend 2006. Frankfurt a. M.: Fischer.

Hynd, C. R., McWorther, J. Y., Phares, V. L., \& Suttles, C. W. (1994). The role of instructional variables in conceptual change. Journal of Research in Science Teaching, 31, 933-946.

IUCN - International Union for the Conservation of Nature and Natural Resources (1980). The World Conservation Strategy. Geneva: IUCN, UNEP, WWF. 
Jäkel, L., \& Schaer, A. (2004). Sind Namen nur Schall und Rauch? Wie sicher sind Pflanzenkenntnisse von Schülerinnen und Schülern? Berichte des Instituts für Didaktik der Biologie, 13, 1-24.

Johann, E. (2002). Socio-economic and forest ecosystem change in Europe. Vienna: International Union of Forestry Research Organizations.

Johnson, C. Y., Bowker, J. M., \& Cordell, K. H. (2004). Ethnic variation in environmental belief and behavior: an examination of the New Ecological Paradigm in a social psychological context. Environment and Behavior, 36, 157-186.

Kahn, M. E. (2006). Green cities: urban growth and the environment. Washington D.C.: Brookings Institution Press.

Kaiser, F. G., Ranney, M., Hartig, T., \& Bowler, P. A. (1999). Ecological Behavior, Environmental Attitude, and Feelings of Responsibility for the Environment. European Psychologist, 4, 59-74.

Kaiser, F. G., \& Shimoda, T. A. (1999). Responsibility as a Predictor of Ecological Behaviour. Journal of Environmental Psychology, 19, 243-253.

Kalof, L., Dietz, T., Guagnano, G. A., \& Stern, P. C. (2002). Race, gender and environmentalism: the atypical values and beliefs of white men. Race, Gender and Class, 9, 1-19.

Kassas, M. (2002). Environmental education: biodiversity. The Environmentalist, 22, 345351.

Kattmann, U., Gropengießer, H., \& Komorek, M. (1997). Das Modell der didaktischen Rekonstruktion - ein Rahmen für naturwissenschaftliche Forschung und Entwicklung. Zeitschrift für Didaktik der Naturwissenschaften, 3, 3-18.

Kerski, J., \& Ross, S. (2005). The environment. London: Hodder Arnold.

Kilchör, N. (2001). Achtung-fertig-Frauen! Interface, 16, 7-9.

Klöckner, C., \& Matthies, E. (2004). How habits interfere with norm-directed behavior: a normative decision-making model for travel mode choice. Journal of Environmental Psychology, 24, 319-327.

Kluge, S. (1999). Empirisch begründete Typenbildung - zur Konstruktion von Typen und Typologien in der qualitativen Sozialforschung. Opladen: Leske und Budrich. 
Kluge, S. (2000). Empirically grounded construction of types and typologies in qualitative social research. Forum Qualitative Sozialforschung / Forum: Qualitative Social Research, 1 (online), http://qualitative-research.net/fqs (accessed at: May, 8th, 2007).

Kopfmüller, J., Brandl, V., Jörissen, J., Pateau, M., Banse, G., Coenen, R., \& Grunwald, A. (2001). Nachhaltige Entwicklung integrativ betrachtet. Konstitutive Elemente, Regeln, Indikatoren. Unpublished manuscript, Berlin, Germany.

Krebs, A. (1997). Naturethik im Überblick. In A. Krebs (Ed.), Naturethik. Grundtexte der gegenwärtigen tier- und ökoethischen Diskussion (pp. 337-380). Frankfurt: Suhrkamp.

Krombaß, A., \& Harms, U. (2006). Ein computergestütztes Informationssystem zur Biodiversität als motivierende und lernförderliche Ergänzung der Exponate eines Naturkundemuseums. Zeitschrift für Didaktik der Naturwissenschaften, 12, 23-44.

Krombaß, A., Urhahne, D., \& Harms, U. (2003). Lernen mit neuen Medien: TREBIS. Entwicklung und Erprobung eines Informationssystems zum Thema Biodiversität. In H. Korn \& U. Feit (Eds.), Treffpunkt Biologische Vielfalt (Vol. III, pp. 201-206). Bonn-Bad Godesberg: BfN.

Kuckartz, U. (2004). MAXqda 2: software for qualitative data analysis (Version 2).

Kyburz-Graber, R. (2006). Studies on a socio-ecological approach to environmental education: a contribution to a critical position in the education for sustainable development discourse. Environmental Education Research, 12, 101-114.

Lindemann-Matthies, P. (1999). Children's perception of biodiversity in everyday life and their preferences for species. Unpublished doctoral dissertation, Zürich University, Switzerland.

Lindemann-Matthies, P. (2002a). Vielfalt am Schulweg. In Umweltdachverband (Ed.), Leben in Hülle und Fülle (pp. 40-44). Wien: Umweltdachverband.

Lindemann-Matthies, P. (2002b). The influence of an educational program on children's perception of biodiversity. The Journal of Environmental Education, 33, 22-31.

Lindemann-Matthies, P. (2006). Investigating nature on the way to school: responses to an educational programme by teachers and their pupils. International Journal of Science Education, 8, 895-910.

Lovejoy, T. E., \& Hannah, L. (2006). Climate change and biodiversity. New Haven, London: Yale University Press. 
Ludwig, G., \& Schnittler, M. (1996). Rote Liste gefährdeter Pflanzen Deutschlands (Vol. 28). Bonn: Bundesamt für Naturschutz.

Lynch, D. (1993). The garden and the sea: U.S. Latino environmental discourses and mainstream environmentalism. Social Problems, 40, 108-124.

Mallow, D. (1994). Biodiversity. The Science Teacher, 61, 19-21.

Marggraf, R., \& Streb, S. (1997). Ökonomische Bewertung der natürlichen Umwelt. Berlin: Springer.

Marshall, N. T. (1998). Searching for a cure - conservation of medical wildlife resources in East and Southern Africa (TRAFFIC Network Report). Cambridge: TRAFFIC.

Mayer, J. (1996). Biodiversitätsforschung als Zukunftsdisziplin. Berichte des Instituts für Didaktik der Biologie, 5, 19-41.

Mayer, J., Harms, U., Hammann, M., Bayrhuber, H., \& Kattmann, U. (2004). Kerncurriculum Biologie der gymnasialen Oberstufe. Mathematischnaturwissenschaftlicher Unterricht, 57, 166-173.

Mayr, E. (2002). What evolution is. London: Weidenfeld \& Nicolson.

McKee, J. K. (2003). Sparing Nature. The conflict between human population growth and earth's biodiversity. New Brunswick, New Jersey, London: Rutgers University Press.

Menzel, S. (2006). Caminos empíricos en la investigación didácitica para mostrar la necesidad de una enseñanza transdisciplinaria sobre la biodiversidad. In $\mathrm{M}$. Quintanella (Ed.), Educación científica, ciudadanía y valores: Retos de los Nuevos Modelos de Conocimiento (CD-ROM). Santiago de Chile: Pontificia Universidad Católica de Chile \& UNESCO.

Menzel, S., \& Bögeholz, S. (2005). Lernvoraussetzungen für Biodiversity Education in Deutschland und Chile am Beispiel endemischer Medizinalpflanzen. In H. Korn \& U. Feit (Eds.), Treffpunkt Biologische Vielfalt (Vol. V, pp. 97-103). Bonn-Bad Godesberg: BfN.

Menzel, S., \& Bögeholz, S. (2006). Vorstellungen und Argumentationsstrukturen von Schüler(inne)n der elften Jahrgangstufe zur Biodiversität, deren Gefährdung und Erhaltung. Zeitschrift für Didaktik der Naturwissenschaften, 12, 199-217.

Menzel, S., \& Bögeholz, S. (submitted a). Was fördert die Bereitschaft von Oberstufenschüler(inne)n, die Biodiversität zu schützen? - Eine quantitative Studie in Anlehnung an die Value-Belief-Norm Theorie. Manuscript submitted for publication. 
Menzel, S., \& Bögeholz, S. (submitted b). The loss of biodiversity as a challenge for sustainable development: How do pupils in Chile and Germany perceive resource dilemmas? Manuscript submitted for publication.

Menzel, S., \& Bögeholz, S. (to be submitted). Values, beliefs and norms that foster Chilean and German students' commitment to protect biodiversity. Manuscript submitted for publication.

Miles, M. B., \& Huberman, M. A. (1994). Qualitative data analysis - an expanded sourcebook (2nd edition). Thousand Oaks: Sage Publications.

Milliken, W., \& Ratter, J. (Eds.). (1998). Maraca: the biodiversity and environment of an Amazonian rainforest. New York, London: Wiley.

Mittermeier, R. A., Robles Gil, P., Hoffmann, M., Pilgrim, J., Brooks, T., Mittermeier, C. G., Lamoreux, J., \& da Fonseca, G. A. B. (2004). Hotspots Revisited. Mexico: CEMEX.

Myers, N. (1985). Tropical deforestation and species extinction; the latest news. Futures: The Journal of Forecasting and Planning, 17, 451-463.

Myers, N., Mittermeier, R. A., Mittermeier, C. G., da Fonseca, G. A. B., \& Kent, J. (2000). Biodiversity hotspots for conservation priorities. Nature, 403, 853-858.

Naess, A. (1997). Die Tiefenökologische Bewegung: einige philosophische Aspekte. In A. Krebs (Ed.), Naturethik. Grundtexte der gegenwärtigen tier- und ökoethischen Diskussion (pp. 182-210). Frankfurt: Suhrkamp.

Niedersächsisches Kultusministerium (2001). Global denken - lokal handeln. Empfehlungen zur Umweltbildung an allgemein bildenden Schulen. Seelze: Kallmeyersche Verlagsbuchhandlung.

Noe, F. P., \& Snow, R. (1990). Hispanic cultural influence on environmental concern. The Journal of Environmental Education, 21, 27-34.

Nordlund, A., \& Garvill, J. (2002). Value structures behind proenvironmental behavior. Environment and Behavior, 34, 740-756.

Olsen, M., E., Lodwick, D. G., \& Dunlap, R. E. (1992). Viewing the world ecologically. Boulder, Colorado: Westview Press.

Oom do Valle, P., Rebelo, E., Reis, E., \& Menezes, J. (2005). Combining behavioral theories to predict recycling involvement. Environment and Behavior, 37, 364-396.

Oreg, S., \& Katz-Gerro, T. (2006). Predicting proenvironmental behaviour cross-nationally. Environment and Behavior, 38, 462-483. 
Pepper, D. (1993). Eco-socialism: from deep ecology to social justice. London: Routledge.

Piaget, J. (1974). Der Aufbau der Wirklichkeit beim Kinde. Stuttgart: Klett.

Pirages, D.C., \& Ehrlich P.R. (1974). Ark II: Social response to environmental imperatives. San Franscisco: Freeman.

du Plessis, M. A. (2001). Academia as a nursery ground for conservation biology. Conservation Biology, 15, 1477-1478.

Posner, G. J., Strike, K. A., Hewson, P. W., \& Gertzog, W. A. (1982). Accomodation of a scientific conception: toward a theory of conceptual change. Science Education, 66, 211-227.

Pounds, A. J., Fogden, M. P. L., \& Campbell, J. H. (1999). Biological response to climate change on a tropical mountain. Nature, 398, 611-615.

Pullin, A. S. (2002). Conservation biology. Cambridge: Cambridge University Press.

Ratcliffe, M., \& Grace, M. (2003). Science education for citizenship. Berkshire: Open University Press.

Redclift, M. (1987). Sustainable development: exploring the contradictions. London: Routledge.

Rieckmann, M. (2004). Lokale Agenda 21 in Chile. München: Ökom Verlag.

Rost, D. H. (2005). Interpretation und Bewertung pädagogisch-pychologischer Studien. Weinheim, Basel: Beltz UTB.

Rychen, S. D., \& Salganik, L. H. (2001). Defining and electing key competencies. Göttingen: Hogrefe \& Huber.

Salinas Hernández, I. S. (2002). El concepto de biodiversidad: un nuevo contenido de ecología en la educación secondaria. Unpublished doctoral dissertation, Universidad de Granada, Spain.

Schmidt-Bleek, F. (2004). Der ökologische Rucksack, Wirtschaft für eine Zukunft mit Zukunft. Stuttgart, Leipzig: S. Hirzel Verlag.

Schmidt-Grunert, K. (1999). Sozialarbeitsforschung konkret. Problemzentrierte Interviews als qualitative Erhebungsmethode. Freiburg: Lambertus.

Scholz, I. (1999). Medio ambiente y competitividad: el caso de exportaciones chilenas. In K. Eßer (Ed.), Competencia global y libertad de acción nacional (pp. 99-116). Caracas: Vozes. 
Schultz, W. P. (2001). The structure of environmental concern: concern for the self, other people, and the biosphere. Journal of Environmental Psychology, 21, 327-339.

Schultz, W. P., Unipan, J. B., \& Gamba, R. J. (2000). Acculturation and ecological worldview among Latino Americans. The Journal of Environmental Education, 31, $22-27$.

Schultz, W. P., \& Zelezny, L. (1998). Values and proenvironmental behaviour. A fivecountry survey. Journal of Cross-Cultural Psychology, 29, 540-558.

Schultz, W. P., \& Zelezny, L. (1999). Values as predictors of environmental attitudes: evidence for consistency across 14 countries. Journal of Environmental Psychology, 19, 255-265.

Schwartz, S. H. (1977). Normative influence on altruism. In L. Berkowitz (Ed.), Advances in experimental psychology (Vol. 10, pp. 222-275). New York, San Fransisco, London: Academic Press.

Schwartz, S. H. (1992). Universal structure of values: theoretical advances and empirical tests in 20 countries. Advances in experimental social psychology, 25, 1-65.

Schwartz, S. H. (1994). Are there universal aspects in the structure and contents of human values? Journal of Social Issues, 50, 19-45.

Schwartz, S. H. (2005). Robustness and fruitfulness of a theory of universals in individual human values. In A. Tamayo \& J. Porto (Eds.), Valores e comportamento nas organizacões (pp. 56-95). Petrópolis: Vozes.

Schwartz, S. H., \& Howard, J. A. (1981). A normative decision-making model of altruism. In P. J. Rushton \& R. M. Sorrentino (Eds.), Altruism and helping behaviour: social, personality, and developmental perspectives (pp. 189-211). Hillsdale: Erlbaum.

Scott, P. H., Asoko, H. M., \& Driver, R. H. (1991). Teaching for conceptual change: a review of strategies. In R. Duit, F. Goldberg \& H. Niederer (Eds.), Research in physics learning: theoretical issues and empirical studies (pp. 310-329). Bremen: IPN.

Shiva, V. (2000). Biodiversität. Bern, Stuttgart, Wien: Verlag Paul Haupt.

Sinclair, A. R. E. (2000). The loss of biodiversity: the sixth great extinction. In C. G. van Kooten, E. H. Bulte \& A. R. E. Sinclair (Eds.), Conserving nature's diversity (pp. 915). Aldershot, Burlington, Singapore, Sidney: Ashgate.

Stanley, G. (2006). Phytosymbiosis and the evolution of modern coral reefs. Science, 312, 857-858. 
Stern, P. C. (2000). Toward a Coherent Theory of Environmentally Significant Behavior. Journal of Social Issues, 56, 407-424.

Stern, P. C., \& Dietz, T. (1994). The value basis of environmental concern. Journal of Social Issues, 50, 65-84.

Stern, P. C., Dietz, T., Abel, T., Guagnano, G. A., \& Kalof, L. (1999). A value-belief-norm theory of support for social movements: the case of environmentalism. Research in Human Ecology, 6, 81-97.

Stern, P. C., Dietz, T., \& Guagnano, G. A. (1995b). The New Ecological Paradigm in socialpsychological context. Environment and Behavior, 27, 723-743.

Stern, P. C., Dietz, T., \& Kalof, L. (1993). Value orientations, gender, and environmental concern. Environment and Behavior, 25, 322-348.

Stern, P. C., Dietz, T., Kalof, L., \& Guagnano, G. A. (1995a). Values, beliefs, and proenvironmental action: attitude formation toward emergent attitude objects. Journal of Applied Social Psychology, 25, 1611-1636.

Strauss, A. L. (1994). Grundlagen qualitativer Sozialforschung. München: UTB.

Strube, G. (1984). Assoziation: der Prozess des Erinnerns und die Struktur des Gedächtnisses. Berlin: Springer.

Tainter, J. A. (2003). Rational sustainablility. World Futures, 59, 213-223.

Tal, T., \& Kedmi, Y. (2006). Teaching socioscientific issues: classroom culture and students' performance. Cultural Studies of Science Education, 1, 615-644.

Terry, A., Hill, J., \& Woodland, W. (2006). Uniting national aspirations and local implementation in sustainable development: an Introduction. In J. Hill \& A. Terry \& W. Woodland (Eds.), Sustainable development: national aspirations, local implementation. Aldershot: Ashgate.

Townsend, C. R., Begon, M. E., \& Harper, J. L. (2003). Essentials of ecology. Oxford: Blackwell.

UNCED - United Nations Conference on Environment and Development (1992). Agenda 21. Rio de Janeiro: UNCED.

UNECE - United Nations Economic and Social Council (2005). Strategy for education for sustainable development (strategy paper). Vilnius: UNECE.

UNEP - United Nations Environmental Programme (1995). Global Biodiversity Assessment. Cambridge: Cambridge University Press. 
UNESCO - United Nations Educational, Scientific and Cultural Organization (2005). UNDekade “ Bildung für nachhaltige Entwicklung” (Resolution der 65. Hauptversammlung der DUK Deutschen UNESCO-Kommission e. V.). Bonn: DUK.

Vikan, A., Camino, C., Biaggio, A., \& Nordvik, H. (2007). Endorsement of the New Ecological Paradigm: a comparison of two Brazilian samples and one Norwegian sample. Environment and Behavior, 39, 217-228.

Vogel, H., Doll, U., Munoz, M., Razmilic, I., San Martin, J., \& Vizcarra, G. (1998). Boldo (Peumus boldus Mol.) - Vermehrungsversuche und ökophysiologische Studien am natürlichen Standort in Chile. Drogenreport, 11, 14-17.

Vosniadou, S. (1992). Knowledge aquisition and conceptual change. Applied Psychology: An International Review, 41, 347-357.

Vosniadou, S. (1994). Capturing and modeling the process of conceptual change. Learning and Instruction, 4, 45-69.

Vosniadou, S. (2001). Conceptual change research and the teaching of science. In $\mathrm{H}$. Behrend (Ed.), Research in science education, past, present, and future (pp. 177-188). Dordrecht, Boston, London: Kluwer Academic Publishers.

Walker, B. H. (1992). Biodiversity and biological redundancy. Conservation Biology, 6, 1823.

Wals, A. E. J., \& Jickling, B. (2002). "Sustainability" in higher education: from doublethink and newsspeak to critical thinking and meaningful learning. International Journal of Sustainability in Higher Education, 3, 221-232.

WCED World Commission on Environment and Development (1987). Our common future. Oxford: Oxford University Press.

van Weelie, D. (2002). Making biodiversity meaningful through environmental education. International Journal of Science Education, 24, 1143-1156.

Weinert, F. E. (2001). Vergleichende Leistungsmessung in Schulen - eine umstrittene Selbstverständlichkeit. In F. E. Weinert (Ed.), Leistungsmessung in Schulen (pp. 1731). Weinheim, Basel: Beltz.

Whitehead, M. (2007). Spaces of sustainability. London: Routledge.

Widegren, Ö. (1998). The New Environmental Paradigm and personal norms. Environment and Behavior, 30, 75-100.

Wilson, E. O. (1992). The diversity of life. New York: Harvard University Press. 
Wilson, E. O. (2001). The diversity of life (2nd edition). London: Penguin.

Witzel, A. (1989). Das problemzentrierte Interview. In G. Jüttemann (Ed.), Qualitative Forschung in der Psychologie - Grundfragen, Verfahrensweisen, Anwendungsfelder (pp. 227-255). Heidelberg: Roland Asanger Verlag.

Witzel, A. (2000). The problem-centered interview. Forum Qualitative Sozialforschung / Forum: Qualitative Social Research, 1 (online), http://www.qualitativeresearch.net/fqs (accessed at: May, 15th, 2007).

Worster, D. (1993). The shaky ground of sustainability. In W. Sachs (Ed.), Global ecology (pp. 132-145). London: Zed Books.

WSSD World Summit on Sustainable Development (2002). The Johannesburg Declaration on sustainable development. Johannesburg: WSSD.

Young, J. (2001). Linking EfS and Biodiversity? A UK-wide survey of the status of education within local biodiversity action plans. Environmental Education Research, 7, 439-449.

von Zerssen, D. (1973). Methoden der Konstitutions- und Typenforschung. In M. Thiel (Ed.), Enzyklopädie der geisteswissenschaftlichen Arbeitsmethoden (pp. 35-143). München, Wien: Oldenbourg. 



\section{Appendix}





\section{Interviewleitfaden \\ Schülervorstellungen zu ökologisch-sozialen Dilemmata im Rahmen der Erhaltung von Biodiversität}

- Hauptstudie 2004 -

Didaktik der Biologie, Zentrum für empirische Unterrichts- und Schulforschung (ZeUS), GEORG-AUGUST-UNIVERSITÄT GÖTTINGEN

\section{Aufbau des Leitfadens:}

\section{- Vorinformationen -}

Block Ia: Allgemeiner Fragehorizont zur Biodiversität

Block Ib: Intervention: Die Teufelskralle: Dilemmadiskussion mit Interventionen

Block II: $\quad$ Boldo - Transferbeispiel

Block III: Eigene Beispiele und Lösungsperspektiven

Block IV: Nachfragen

\section{Vorinformation zum Interview - deutsche Stichprobe}

Ich freue mich, dass Du Dir die Zeit nimmst, heute mit mir dieses Interview zu führen. Im Rahmen meiner Doktorarbeit untersuche ich Schülervorstellungen.

Um herauszufinden, was Schülerinnen und Schüler denken, werden in der Forschung oft Interviews geführt. Ziel ist es, den Biologie-Unterricht zu verbessern und Verstehen von Biologie zu fördern. Dazu ist die Kenntnis der Vorstellungen von Schülerinnen und Schülern hilfreich. Nur wenn Lehrerinnen und Lehrer die Vorstellungen von Schülerinnen und Schülern kennen, können sie an deren Vorstellungen anknüpfen. So können leichter fruchtbare Lernprozesse eingeläutet werden.

In diesem Interview geht es darum, deine persönlichen Vorstellungen zu einem Themenkomplex der Biologie zu erfahren. Für uns ist es hilfreich zu wissen, wie Schülerinnen und Schüler denken. Alle Antworten sind somit echte Informationen für uns. Dabei gibt es kein „richtig“ oder „falsch“, denn es ist Ziel herauszufinden, wo Unterricht ansetzen sollte.

Es kann sein, dass ich öfter mal genauer nachfrage, damit ich Deine Vorstellungen auch wirklich verstehen kann. Du würdest uns am meisten helfen, wenn Du einfach offen das sagst, was dir zu unseren Fragen einfällt. Selbstverständlich wird alles vertraulich und anonym behandelt, was wir hier besprechen. Auch an Deine Lehrerinnen und Lehrer werden keine Informationen weitergegeben. Damit keine Rückschlüsse auf Deine Person möglich sind, wird Dein Name geändert. 


\title{
Guía de Entrevista \\ Ideas de Alumnos de dilemas ecologicales-sociales en el marco de sostenimiento de la biodiversidad
}

\author{
- Investigación cualitativo 2004 - \\ Programa de Magíster en Educación de las Ciencias \\ UNIVERSIDAD DE TALCA, CHILE \\ Didáctica de la Biología, Centro de Investigación Empirico en Escuela y Enseñanza \\ (ZeUS), \\ GEORG-AUGUST-UNIVERSITÄT GÖTTINGEN, ALEMANIA
}

Susanne Menzel, Olga Contreras Perez \& Susanne Bögeholz ${ }^{1}$

\section{Informaciónes para el alumno antes del entravista}

Me alegro mucho que te has tomado el tiempo ahora para hacer eso entravista conmigo. Investigo las ideas de alumnos en el marco de mi tesis de Magíster. La investigación de ideas de alumnos es una especialidad de investigación en la didáctica.

Para conocer que alumnos piensen y saben, es el uso general en las ciencias de la diáctica de hacer entrevistas. Es el objetivo de ese tipo de investigación, de mejorar la enseñanza de la biología en la escuela. Por eso, el conociemento de las ideas de los alumnos es muy importante y benefico. Solo cuando los profesores y las profesoras conozcen las ideas de alumnos, pueden anudar en las ideas que ya existen así que dar una enseñanza effectivo y criadera.

En este entrevista queremos que conocer tus ideas propias sobre un tema de la biología. Para nosotras, es muy útil de saber que y como alumnos y alumnas piensen sobre el tema. Todas tus respuestas son informaciónes ciertas para nosotros. El acierto o falsía no existe en este caso. Es nuestro objetivo de aprender donde la enseñanza empezaría.

Es posible que insistiré a veces, porque quiero a entender tus respuestas realmente. Tu me ayudaras lo más si me digas muy sincero que tu pienses y que te acude a la mente como respuesta por nos preguntas.

Por supuesto todo que dices va a ser tratado anónima rígitadamente. Tus profesores también no pueden obtenir las informaciones que tu nos diste. A fin de que no relaciónes contigo seran posible, tu nombre sera modificado.

\footnotetext{
${ }^{1}$ Siguiendo en la guía de entrevista de Helmers, Bögeholz, Menzel (2004)
} 


\section{Block Ia: Allgemeiner Fragehorizont zur Biodiversität}

a. Begrifflichkeit

\begin{tabular}{|c|c|c|c|}
\hline $\begin{array}{l}\text { Forschungs- } \\
\text { interesse }\end{array}$ & $\begin{array}{l}\text { Frage- } \\
\text { interventionen }\end{array}$ & Intervención & Erwartungshorizont \\
\hline $\begin{array}{l}\text { Kennen } \\
\text { Schülerinnen und } \\
\text { Schüler (SuS) den } \\
\text { Begriff } \\
\text { „Biodiversität““ } \\
\text { oder den Begriff } \\
\text { „biologische } \\
\text { Vielfalt“? }\end{array}$ & $\begin{array}{l}\text { 1. Was verbindest } \\
\text { Du mit „biologischer } \\
\text { Vielfalt“? } \\
\text { 1.a Und mit dem } \\
\text { Begriff } \\
\text { „Biodiversität“? }\end{array}$ & $\begin{array}{l}\text { 1. ¿Qué asocias con } \\
\text { el término diversidad } \\
\text { biológica? } \\
\text { 1.a ¿Y con el } \\
\text { término } \\
\text { „biodiversidad“? }\end{array}$ & $\begin{array}{l}\text { Biologische Vielfalt } \\
\text { kann alltagssprachlich } \\
\text { hergeleitet werden, } \\
\text { Biodiversität ist } \\
\text { manchen SuS } \\
\text { unbekannt: Begriff } \\
\text { dann klären vor } \\
\text { Fortsetzung des } \\
\text { Interviews! }\end{array}$ \\
\hline
\end{tabular}

b. Verbreitung biologischer Vielfalt

\begin{tabular}{|c|c|c|c|}
\hline $\begin{array}{l}\text { Forschungs- } \\
\text { interesse }\end{array}$ & $\begin{array}{l}\text { Frage- } \\
\text { interventionen }\end{array}$ & Intervención & Erwartungshorizont \\
\hline $\begin{array}{l}\text { Weitere } \\
\text { Auskünfte zu } \\
\text { Vorstellungen, die } \\
\text { SuS zu } \\
\text { biologischer } \\
\text { Vielfalt haben: } \\
\text { Regionales oder } \\
\text { globales } \\
\text { Phänomen? }\end{array}$ & $\begin{array}{l}\text { 2. Wie stellt sich die } \\
\text { biologische Vielfalt } \\
\text { in den verschiedenen } \\
\text { Regionen der Welt } \\
\text { dar? }\end{array}$ & $\begin{array}{l}\text { 2. ¿Cómo es la } \\
\text { diversidad biológica } \\
\text { diseminado en el } \\
\text { mundo? }\end{array}$ & $\begin{array}{l}\text { Gradient biologischer } \\
\text { Vielfalt vom Äquator } \\
\text { zu den Polen } \\
\text { abnehmend. } \\
\text { Tropischer Regenwald } \\
\text { ist Ort hoher } \\
\text { biologischer Vielfalt. }\end{array}$ \\
\hline $\begin{array}{l}\text { Wenn SuS } \\
\text { biologische } \\
\text { Vielfalt } \\
\text { beschreiben, } \\
\text { verwenden sie den } \\
\text { Begriff } \\
\text { „biologische } \\
\text { Vielfalt“ im } \\
\text { Kontext } \\
\text { zutreffend? }\end{array}$ & & & \\
\hline $\begin{array}{l}\text { Nachfrage zur } \\
\text { weiteren Klärung }\end{array}$ & $\begin{array}{l}\text { 2.a Welches Bild } \\
\text { hast Du von Orten } \\
\text { mit hoher } \\
\text { biologischer Vielfalt? }\end{array}$ & $\begin{array}{l}\text { 2.a ¿Cómo te } \\
\text { imagines lugares con } \\
\text { una grande variedad } \\
\text { biológica? }\end{array}$ & \\
\hline
\end{tabular}




\section{c. Aspekte der Evolution und Ökologie}

\begin{tabular}{|l|l|l|l|}
\hline \multicolumn{1}{|c|}{$\begin{array}{c}\text { Forschungs- } \\
\text { interesse }\end{array}$} & \multicolumn{1}{|c|}{$\begin{array}{c}\text { Frage- } \\
\text { interventionen }\end{array}$} & \multicolumn{1}{|c|}{ Intervención } & Erwartungshorizont \\
\hline $\begin{array}{l}\text { Können SuS } \\
\text { sehen, dass } \\
\text { biologische } \\
\text { Vielfalt auf } \\
\text { verschiedenen } \\
\begin{array}{l}\text { Ebenen existiert } \\
\text { und mit Ökologie } \\
\text { und Evolution eng } \\
\text { verbunden ist? }\end{array}\end{array}$ & $\begin{array}{l}\text { 3. Wovon hängt es } \\
\text { ab, dass biologische } \\
\text { Vielfalt in } \\
\text { verschiedenen } \\
\text { Facetten vorkommt? }\end{array}$ & $\begin{array}{l}\text { 3. ¿De qué depende } \\
\text { que la diversidad } \\
\text { biológica ocurre en } \\
\text { facetas diferentes ? }\end{array}$ & $\begin{array}{l}\text { - Rolle des Menschen } \\
\text { - evolutionäre } \\
\text { Entwicklung } \\
\text { - geographische } \\
\text { Einflüsse (Wüste etc.) }\end{array}$ \\
\hline
\end{tabular}

\section{d. Interdisziplinäre Aspekte: Der Wert biologischer Vielfalt}

\begin{tabular}{|c|c|c|c|}
\hline $\begin{array}{l}\text { Forschungs- } \\
\text { interesse }\end{array}$ & $\begin{array}{l}\text { Frage- } \\
\text { interventionen }\end{array}$ & Intervención & Erwartungshorizont \\
\hline $\begin{array}{l}\text { Können SuS das } \\
\text { Phänomen } \\
\text { Biodiversität } \\
\text { außerhalb des } \\
\text { naturwissenschaft- } \\
\text { lichen } \\
\text { Bezugsrahmens } \\
\text { betrachten? }\end{array}$ & $\begin{array}{l}\text { 4. Aus welchen } \\
\text { Gründen könnte } \\
\text { biologische Vielfalt } \\
\text { wertvoll sein? }\end{array}$ & $\begin{array}{l}\text { 4. ¿Porqué podía la } \\
\text { variedad biológica } \\
\text { estar de valor? }\end{array}$ & \multirow[t]{2}{*}{$\begin{array}{l}\text { Unterschiedliche } \\
\text { Werte der } \\
\text { biologischen Vielfalt: } \\
\text { Z.B. intrinsischer, } \\
\text { inhärenter, } \\
\text { ästhetischer oder } \\
\text { instrumenteller Wert. }\end{array}$} \\
\hline $\begin{array}{l}\text { Nachfrage zur } \\
\text { Verstärkung }\end{array}$ & $\begin{array}{l}\text { 4.a In welchem } \\
\text { Zusammenhang } \\
\text { spielt biologische } \\
\text { Vielfalt noch eine } \\
\text { Rolle? }\end{array}$ & $\begin{array}{l}\text { 4.a ¿En qué contexto } \\
\text { más tiene la variedad } \\
\text { biológica un papel ? }\end{array}$ & \\
\hline $\begin{array}{l}\text { Ist SuS die } \\
\text { ökonomische } \\
\text { Bedeutung von } \\
\text { Naturprodukten } \\
\text { bewusst? } \\
\text { Erkennen SuS } \\
\text { Biodiversität als } \\
\text { Teil der eigenen } \\
\text { Lebenswirklichkei } \\
\text { t durch Nutztiere } \\
\text { und -pflanzen? }\end{array}$ & $\begin{array}{l}\text { 4.b Für wen und } \\
\text { aus welchen } \\
\text { Gründen kann } \\
\text { biologische Vielfalt } \\
\text { von Nutzen sein? }\end{array}$ & $\begin{array}{l}\text { 4.b ¿Para quién y } \\
\text { porque puede la } \\
\text { variedad biológica } \\
\text { estar importante? }\end{array}$ & $\begin{array}{l}\text { Nennung von } \\
\text { Rohstoffen und } \\
\text { Materialien zur } \\
\text { industriellen } \\
\text { Produktion, die der } \\
\text { Natur entstammen } \\
\text { (Nahrung, } \\
\text { Arzneimittel, } \\
\text { Kosmetika, Kleidung, } \\
\text { Baustoffe etc.) }\end{array}$ \\
\hline
\end{tabular}


e) Produkte des täglichen Lebens - Zusammenhang mit biologischer Vielfalt Intervention: Aufforderung, den Bezug zum eigenen Leben herzustellen

\begin{tabular}{|c|c|c|c|}
\hline $\begin{array}{l}\text { Forschungs- } \\
\text { interesse }\end{array}$ & $\begin{array}{l}\text { Frage- } \\
\text { interventionen }\end{array}$ & Intervención & Erwartungshorizont \\
\hline $\begin{array}{l}\text { Sehen SuS, dass } \\
\text { sie direkt von } \\
\text { Produkten } \\
\text { biologischer } \\
\text { Vielfalt } \\
\text { profitieren? }\end{array}$ & 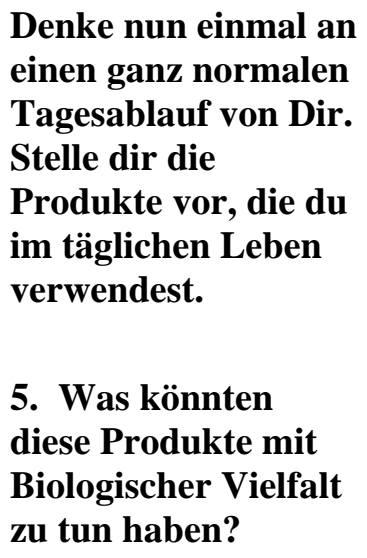 & $\begin{array}{l}\text { Imaginate un } \\
\text { transcurso del día, } \\
\text { que es normal para } \\
\text { ti. Prestentate los } \\
\text { productos que } \\
\text { utilizas en la rutina } \\
\text { díaria. } \\
\\
5 . \text { ¿Qué pueden estos } \\
\text { productos tener que } \\
\text { ver con la variedad } \\
\text { biologica? }\end{array}$ & $\begin{array}{l}\text { Nahrung, } \\
\text { Pflegeprodukte des } \\
\text { täglichen Lebens } \\
\text { werden aus } \\
\text { Ressourcen } \\
\text { biologischer Vielfalt } \\
\text { hergestellt. }\end{array}$ \\
\hline
\end{tabular}

f) Intervention: Präsentation von Produkten: Ökonomischer Nutzen und Übernutzung biologischer Vielfalt

\begin{tabular}{|c|c|c|c|}
\hline $\begin{array}{l}\text { Forschungs- } \\
\text { interesse }\end{array}$ & $\begin{array}{l}\text { Frage- } \\
\text { interventionen }\end{array}$ & Intervención & Erwartungshorizont \\
\hline $\begin{array}{l}\text { Ist SuS bewusst, } \\
\text { dass biologische } \\
\text { Vielfalt durch } \\
\text { konventionelle } \\
\text { Vermarktung } \\
\text { gefährdet sein } \\
\text { kann? }\end{array}$ & $\begin{array}{l}\text { Ich habe eine Reihe } \\
\text { von pflanzlichen } \\
\text { Produkten } \\
\text { mitgebracht. }\end{array}$ & $\begin{array}{l}\text { He llevado alogunos } \\
\text { productos vegetales. } \\
\text { 6. ¿Puedes } \\
\text { imaginarte que } \\
\text { algunos de estos } \\
\text { productos conminen } \\
\text { la variedad } \\
\text { biológica? }\end{array}$ & $\begin{array}{l}\text { Gefährdung von } \\
\text { biologischer Vielfalt } \\
\text { durch eine } \\
\text { Übernutzung } \\
\text { natürlicher } \\
\text { Ressourcen in } \\
\text { Wildsammlung. }\end{array}$ \\
\hline $\begin{array}{l}\text { Kann } \\
\text { Wildsammlung } \\
\text { nach Hinweis als } \\
\text { Gefährdungsgrund } \\
\text { angesehen } \\
\text { werden? }\end{array}$ & $\begin{array}{l}\text { 6.b Welche } \\
\text { Bedeutung könnte es } \\
\text { haben, dass manche } \\
\text { dieser Produkte aus } \\
\text { Wildsammlung } \\
\text { stammen? }\end{array}$ & $\begin{array}{l}\text { 6.b ¿Qué puede } \\
\text { significar que } \\
\text { algunos de este } \\
\text { productos estan } \\
\text { colectados de la } \\
\text { selva? }\end{array}$ & $\begin{array}{l}\text { Übernutzung durch } \\
\text { Wildsammlung wird } \\
\text { spätestens hier } \\
\text { erkannt. }\end{array}$ \\
\hline
\end{tabular}




\begin{tabular}{|c|c|c|c|}
\hline $\begin{array}{l}\text { Forschungs- } \\
\text { interesse }\end{array}$ & $\begin{array}{l}\text { Frage- } \\
\text { interventionen }\end{array}$ & Intervención & Erwartungshorizont \\
\hline $\begin{array}{l}\text { Ist SuS bewusst, } \\
\text { dass die Erhaltung } \\
\text { der Biodiversität } \\
\text { in manchen } \\
\text { Zentren der } \\
\text { Biodiversität } \\
\text { („Hotspots“) } \\
\text { besonders } \\
\text { schwierig ist? }\end{array}$ & $\begin{array}{l}\text { 6.c An welchen } \\
\text { Stellen auf der Erde } \\
\text { ist eine Bewahrung } \\
\text { der biologischen } \\
\text { Vielfalt wohl am } \\
\text { schwierigsten? }\end{array}$ & $\begin{array}{l}\text { 6.c ¿En qué lugares } \\
\text { del mundo es la } \\
\text { sostenimiento de la } \\
\text { variedad biológica } \\
\text { más difícil? }\end{array}$ & $\begin{array}{l}\text { Hotspots werden } \\
\text { umschrieben: Z.B. als } \\
\text { arme Länder mit } \\
\text { hoher Biodiversität } \\
\text { und hohem } \\
\text { Bevölkerungswachs- } \\
\text { tum. } \\
\text { Gefährdung der } \\
\text { Biodiversität zur } \\
\text { Befriedigung von } \\
\text { Grundbedürfnissen. }\end{array}$ \\
\hline $\begin{array}{l}\text { Sehen die } \\
\text { Befragten, dass } \\
\text { sozialökonomisch } \\
\text { e Faktoren einen } \\
\text { Einfluss auf den } \\
\text { Zustand der Natur } \\
\text { haben? }\end{array}$ & $\begin{array}{l}\text { 6.d Worin liegen die } \\
\text { Ursachen? }\end{array}$ & $\begin{array}{l}\text { 6.d ¿Qué es el } \\
\text { porqué? }\end{array}$ & - offen - \\
\hline
\end{tabular}




\section{Block Ib: Intervention: Die Teufelskralle: Dilemmadiskussion mit Interventionen}

a) allgemeine Hinführung

\begin{tabular}{|c|c|c|c|}
\hline $\begin{array}{l}\text { Forschungs- } \\
\text { interesse }\end{array}$ & $\begin{array}{l}\text { Frage- } \\
\text { interventionen }\end{array}$ & Intervención & Erwartungshorizont \\
\hline $\begin{array}{l}\text { Verknüpfen SuS } \\
\text { soziale, ökologische } \\
\text { und ökonomische } \\
\text { Aspekte mit dem } \\
\text { „Teufelskrallen- } \\
\text { Dilemma“? }\end{array}$ & $\begin{array}{l}\text { Die Teufelskralle, die } \\
\text { nur im Süden } \\
\text { Afrikas wächst, gilt } \\
\text { als im Bestand stark } \\
\text { gefährdet. } \\
\text { 7. Woran kann das } \\
\text { liegen? }\end{array}$ & $\begin{array}{l}\text { La „Garra del } \\
\text { Diabolo“ es una } \\
\text { planta que sólo } \\
\text { crece en la } \\
\text { Suráfrica y es muy } \\
\text { peligroso. } \\
\\
\text { 7. ¿Puedes } \\
\text { imaginarte por } \\
\text { que? }\end{array}$ & $\begin{array}{l}\text { Finanzielle Not } \\
\text { bewirkt, dass } \\
\text { Naturressourcen } \\
\text { übernutzt werden. } \\
\text { Einzelne haben } \\
\text { kurzfristig Gewinn, } \\
\text { Schaden wird von der } \\
\text { Gemeinschaft } \\
\text { getragen }\end{array}$ \\
\hline
\end{tabular}

b) soziale Aspekte

\begin{tabular}{|l|l|l|l|}
\hline \multicolumn{1}{|c|}{$\begin{array}{c}\text { Forschungs- } \\
\text { interesse }\end{array}$} & \multicolumn{1}{|c|}{$\begin{array}{c}\text { Frage- } \\
\text { interventionen }\end{array}$} & \multicolumn{1}{c|}{ Intervención } & Erwartungshorizont \\
\hline $\begin{array}{l}\text { Verfügen } \\
\text { Schülerinnen und } \\
\text { Schüler über die } \\
\begin{array}{l}\text { Fähigkeit zur } \\
\text { Perspektivübernah } \\
\text { me? }\end{array}\end{array}$ & $\begin{array}{l}\text { 8. Welches Interesse } \\
\text { haben Menschen } \\
\text { daran, Pflanzen zu } \\
\text { ernten und zu } \\
\text { verkaufen, obwohl die } \\
\text { entsprechende Art } \\
\text { bedroht ist? }\end{array}$ & $\begin{array}{l}\text { 8. ¿Qué interes } \\
\text { tiene la gente } \\
\text { recoger y vender } \\
\text { plantas aúnque la } \\
\text { especie está } \\
\text { peligroso? }\end{array}$ & $\begin{array}{l}\text { Ökonomischer Zwang, } \\
\text { Unwissenheit }\end{array}$ \\
\hline
\end{tabular}




\begin{tabular}{|c|c|c|c|}
\hline $\begin{array}{l}\text { Forschungs- } \\
\text { interesse }\end{array}$ & $\begin{array}{l}\text { Frage- } \\
\text { interventionen }\end{array}$ & Intervención & Erwartungshorizont \\
\hline $\begin{array}{l}\text { Haben SuS die } \\
\text { Fähigkeit, die } \\
\text { Situation der } \\
\text { Teufelskrallen- } \\
\text { sammler(innen) } \\
\text { nachzuvollziehen } \\
\text { und nach Wegen } \\
\text { aus dem Dilemma } \\
\text { zu suchen? }\end{array}$ & $\begin{array}{l}\text { 8.a Welche } \\
\text { Zusammenhänge } \\
\text { müssen die } \\
\text { Sammlerinnen und } \\
\text { Sammler der } \\
\text { Teufelskralle } \\
\text { verstehen, um die } \\
\text { Teufelskralle zu } \\
\text { schonen? }\end{array}$ & $\begin{array}{l}\text { 8.a ¿Qué contextos } \\
\text { tienen las } \\
\text { collectores que } \\
\text { entender para } \\
\text { cuidar la „Garra } \\
\text { del Diabolo“? }\end{array}$ & $\begin{array}{l}\text { Verhalten der } \\
\text { Teufelskrallen- } \\
\text { Sammler(innen) } \\
\text { erscheint aus deren } \\
\text { Perspektive logisch, } \\
\text { können die Folgen des } \\
\text { Handelns können } \\
\text { nicht erkannt werden. } \\
\text { Nachhaltige Nutzung } \\
\text { kann eine } \\
\text { Ressourcenüber- } \\
\text { nutzung vermeiden } \\
\text { (intergenerationale } \\
\text { Gerechtigkeit). } \\
\text { Bildung ist nötig, aber } \\
\text { auch die Aufklärung } \\
\text { der Verbraucher über } \\
\text { die Folgen von } \\
\text { Konsumverhalten. }\end{array}$ \\
\hline $\begin{array}{l}\text { Welche } \\
\text { Nutzergruppen } \\
\text { werden erkannt? } \\
\text { Erfolgen } \\
\text { Schuldzuweisunge } \\
\text { n? }\end{array}$ & $\begin{array}{l}\text { 8.b Wer ist für den } \\
\text { Rückgang der Pflanze } \\
\text { verantwortlich zu } \\
\text { machen? }\end{array}$ & $\begin{array}{l}\text { 8.b ¿Quién es } \\
\text { responsable por la } \\
\text { pérdita de la } \\
\text { planta? }\end{array}$ & $\begin{array}{l}\text { Sammler(innen) oder } \\
\text { Industrie. }\end{array}$ \\
\hline $\begin{array}{l}\text { Sind SuS in der } \\
\text { Lage, die } \\
\text { Strukturen des } \\
\text { Teufelskralle- } \\
\text { Dilemmas zu } \\
\text { erkennen? }\end{array}$ & $\begin{array}{l}\text { 8.c Würdest du bitte } \\
\text { mit eigenen Worten die } \\
\text { wichtigsten Probleme } \\
\text { bezüglich der } \\
\text { Teufelskralle } \\
\text { wiedergeben und dabei } \\
\text { die Hauptbeteiligten } \\
\text { nennen? } \\
\text { Begründe bitte, warum } \\
\text { dies die } \\
\text { Hauptbeteiligten sind. } \\
\text { Warum ist die } \\
\text { Situation so } \\
\text { ausgesprochen } \\
\text { schwierig? }\end{array}$ & $\begin{array}{l}\text { 8.c Por favor, } \\
\text { ¿puedes reproducir } \\
\text { con tus palabras } \\
\text { propias los } \\
\text { problemas los más } \\
\text { importantes en } \\
\text { relación con la } \\
\text { „Garra del } \\
\text { Diabolo“ y calificar } \\
\text { las participantes } \\
\text { más importantes? } \\
\text { Funda por qué los } \\
\text { son las } \\
\text { participantes más } \\
\text { importantes. } \\
\text { ¿Por qué es la } \\
\text { situación si difícil ? }\end{array}$ & $\begin{array}{l}\text { Die Vermarktung von } \\
\text { Naturressourcen } \\
\text { fördert deren } \\
\text { Übernutzung. Die } \\
\text { Folgen der } \\
\text { Gefährdung werden } \\
\text { zunächst nicht } \\
\text { erkannt. Die } \\
\text { ökonomische } \\
\text { Betroffenheit } \\
\text { verhindert zudem ein } \\
\text { rationales Vorgehen } \\
\text { und fördert das } \\
\text { Streben nach } \\
\text { kurzfristigem Gewinn } \\
\text { zu Ungunsten einer } \\
\text { schlechteren } \\
\text { Langzeitprognose. }\end{array}$ \\
\hline $\begin{array}{l}\text { Auf welche } \\
\text { Aspekte des } \\
\text { Dilemmas werden } \\
\text { Lösungen } \\
\text { bezogen? }\end{array}$ & $\begin{array}{l}\text { 8.d Welche } \\
\text { Lösungsmöglichkeiten } \\
\text { könntest Du Dir } \\
\text { vorstellen? } \\
\text { Begründe! }\end{array}$ & $\begin{array}{l}\text { 8.d ¿Qué } \\
\text { soluciónes puedes } \\
\text { imaginarte? } \\
\text { ¡Funda! }\end{array}$ & - offen - \\
\hline
\end{tabular}


c) eigene Beispiele / Parallelen

\begin{tabular}{|c|c|c|c|}
\hline $\begin{array}{l}\text { Forschungs- } \\
\text { interesse }\end{array}$ & $\begin{array}{l}\text { Frage- } \\
\text { interventionen }\end{array}$ & Intervención & Erwartungshorizont \\
\hline \multirow[t]{2}{*}{$\begin{array}{l}\text { Können SuS die } \\
\text { erkannten } \\
\text { Strukturen auf } \\
\text { andere Beispiele } \\
\text { übertragen? }\end{array}$} & $\begin{array}{l}\text { 9. Kann man die } \\
\text { Wirkungszusammenhä } \\
\text { nge verallgemeinern? }\end{array}$ & $\begin{array}{l}\text { 9. ¿Es posibile que } \\
\text { se puede } \\
\text { generalizar las } \\
\text { relaciónes de } \\
\text { efectos? }\end{array}$ & - offen - \\
\hline & $\begin{array}{l}\text { 9.a Kennst Du } \\
\text { Beispiele aus } \\
\text { Deutschland oder aus } \\
\text { der Welt, die dem der } \\
\text { Teufelskralle ähneln? }\end{array}$ & $\begin{array}{l}\text { 9.a ¿Conoczes } \\
\text { ejemplos análogos } \\
\text { con la „Garra de } \\
\text { Diabolo“ de Chile o } \\
\text { del mundo? }\end{array}$ & $\begin{array}{l}\text { Bezug auf lokale und } \\
\text { internationale } \\
\text { Beispiele }\end{array}$ \\
\hline
\end{tabular}

\section{Block II: Boldo - Transferbeispiel}

a) spontaner Transfer

\begin{tabular}{|c|c|c|c|}
\hline $\begin{array}{l}\text { Forschungs- } \\
\text { interesse }\end{array}$ & $\begin{array}{l}\text { Frage- } \\
\text { interventionen }\end{array}$ & Intervención & Erwartungshorizont \\
\hline $\begin{array}{l}\text { Werden Parallelen } \\
\text { spontan erkannt? } \\
\text { Welche Aspekte } \\
\text { des vorangehenden } \\
\text { Beispiels } \\
\text { (Teufelskralle) } \\
\text { können transferiert } \\
\text { werden? } \\
\text { Fallen } \\
\text { Schüler(innen) auf } \\
\text { ihre ursprünglichen } \\
\text { Argumentationsmu } \\
\text { ster zurück? }\end{array}$ & $\begin{array}{l}\text { Kommen wir zu Boldo- } \\
\text { Blättern. Diese } \\
\text { stammen aus } \\
\text { Wildsammlung. } \\
\text { 10. Könntest du dir } \\
\text { vorstellen, welche } \\
\text { Probleme durch die } \\
\text { Gewinnung, Nutzung } \\
\text { und Vermarktung von } \\
\text { Boldo entstehen? }\end{array}$ & $\begin{array}{l}\text { Hablamos sobre las } \\
\text { hojas de Boldo. } \\
\text { Originen de la } \\
\text { colleción del sielva. } \\
\text { 10. ¿Puedes } \\
\text { imaginarte qué } \\
\text { problemas surgien } \\
\text { a causa de la } \\
\text { obtención, } \\
\text { utilisación y } \\
\text { comercialización } \\
\text { de Boldo? }\end{array}$ & $\begin{array}{l}\text { Rückgriff auf } \\
\text { Komponenten des } \\
\text { Teufelskrallen- } \\
\text { Dilemmas }\end{array}$ \\
\hline
\end{tabular}


b) soziale Komponenten

\begin{tabular}{|c|c|c|c|}
\hline $\begin{array}{l}\text { Forschungs- } \\
\text { interesse }\end{array}$ & $\begin{array}{l}\text { Frage- } \\
\text { interventionen }\end{array}$ & Intervención & Erwartungshorizont \\
\hline $\begin{array}{l}\text { Wird die } \\
\text { ökonomische } \\
\text { Zwangslage der } \\
\text { Sammler(innen) } \\
\text { erkannt? } \\
\text { Welche } \\
\text { Nutzergruppen } \\
\text { werden als } \\
\text { Schuldige } \\
\text { identifiziert? }\end{array}$ & $\begin{array}{l}\text { 11. Wie bewertest du } \\
\text { das Verhalten der } \\
\text { einzelnen Beteiligten? }\end{array}$ & $\begin{array}{l}\text { 11. ¿Cómo valoras } \\
\text { el comportamiento } \\
\text { de las personas } \\
\text { participados? }\end{array}$ & $\begin{array}{l}\text { Wertung des } \\
\text { Verhaltens als } \\
\text { schuldig/unschuldig } \\
\text { wird vorgenommen }\end{array}$ \\
\hline
\end{tabular}

\title{
Block III: Eigene Beispiele und Lösungsperspektiven
}

\author{
a) Nennung von Dilemmata
}

\begin{tabular}{|l|l|l|l|}
\hline \multicolumn{1}{|c|}{$\begin{array}{c}\text { Forschungs- } \\
\text { interesse }\end{array}$} & \multicolumn{1}{|c|}{$\begin{array}{c}\text { Frage- } \\
\text { interventionen }\end{array}$} & \multicolumn{1}{|c|}{ Intervención } & Erwartungshorizont \\
\hline $\begin{array}{l}\text { Wurde die } \\
\text { Dilemmastruktur } \\
\text { verstanden? => Sind } \\
\text { die genannten }\end{array}$ & $\begin{array}{l}\text { 12. Kennst Du } \\
\text { Beispiele, in denen der } \\
\text { Handel und Verkauf } \\
\text { Beispiele adäquat, } \\
\text { um onkologimisch- } \\
\text { soziale Dilemmata zu } \\
\text { (Tiere oder Pflanzen) } \\
\text { den Bestand reduziert } \\
\text { beschreiben? }\end{array}$ & $\begin{array}{l}\text { 12. ¿Conozces } \\
\text { ejemplos dónde la } \\
\text { comercialización y } \\
\text { la venta de ciertos } \\
\text { especies (Plantas o } \\
\text { animales) han } \\
\text { reducionido la } \\
\text { población? }\end{array}$ & $\begin{array}{l}\text { Elfenbeinhandel, } \\
\text { Walfang, } \\
\text { Argentinisches } \\
\text { Rindfleisch, } \\
\text { Thunfisch, Pflanzen, } \\
\text { die nun unter } \\
\text { strengem Naturschutz } \\
\text { stehen }\end{array}$ \\
\hline
\end{tabular}


b) Lösungsperspektiven

\begin{tabular}{|c|c|c|c|}
\hline $\begin{array}{l}\text { Forschungs- } \\
\text { interesse }\end{array}$ & $\begin{array}{l}\text { Frage- } \\
\text { interventionen }\end{array}$ & Intervención & Erwartungshorizont \\
\hline $\begin{array}{l}\text { Welche Beispiele } \\
\text { sind SuS bekannt? }\end{array}$ & $\begin{array}{l}\text { 13. Kennst Du } \\
\text { Lösungsansätze für } \\
\text { diese Probleme? Wenn } \\
\text { ja, woher? }\end{array}$ & $\begin{array}{l}\text { 13. ¿Conozces } \\
\text { solutiones para este } \\
\text { problemas? } \\
\text { Cuando sí, ¿De } \\
\text { dónde tienes la } \\
\text { información? }\end{array}$ & $\begin{array}{l}\text { Naturschutzgebiete, } \\
\text { Verbote usw. }\end{array}$ \\
\hline $\begin{array}{l}\text { Sind SuS in der } \\
\text { Lage, adäquate } \\
\text { Lösungsstrategien } \\
\text { vorzuschlagen? } \\
\text { Welche Aspekte von } \\
\text { ökologisch-sozialen } \\
\text { Dilemmata greifen } \\
\text { SuS an dieser Stelle } \\
\text { wieder auf? } \\
\\
\text { Beziehen SuS } \\
\text { ökologische, } \\
\text { ökonomische und } \\
\text { soziale Aspekte } \\
\text { gleichermaßen mit } \\
\text { ein? }\end{array}$ & $\begin{array}{l}\text { 13.a Wie sollten } \\
\text { Maßnahmen zum } \\
\text { Schutz von } \\
\text { Biologischer Vielfalt } \\
\text { angelegt sein? } \\
\text { 13.b Welche } \\
\text { Maßnahmen, an die } \\
\text { Du vielleicht auch noch } \\
\text { denkst, würden } \\
\text { vermutlich nicht } \\
\text { helfen? } \\
\text { 13.c Welche } \\
\text { Maßnahmen hältst Du } \\
\text { für am geeignetsten? } \\
\text { 13.d Welche } \\
\text { Maßnahmen, an die } \\
\text { man auch denken } \\
\text { könnte, würden } \\
\text { vermutlich nicht } \\
\text { helfen? }\end{array}$ & $\begin{array}{l}\text { 13.a ¿Cómo deben } \\
\text { meditas } \\
\text { preventivas para la } \\
\text { proteción de la } \\
\text { variedad biologica } \\
\text { estar? } \\
\text { 13.b ¿Qué } \\
\text { meditas, que } \\
\text { puedes considerar } \\
\text { también, no } \\
\text { ayudarían } \\
\text { probablemente? } \\
\\
\text { 13.c ¿Qué pienses, } \\
\text { qué meditas son las } \\
\text { más efectivos? } \\
\text { 13.d ¿Qué } \\
\text { meditas, que se } \\
\text { puede considerar } \\
\text { también, no } \\
\text { ayudarían } \\
\text { probablemente? }\end{array}$ & $\begin{array}{l}\text { Nachhaltige Nutzung } \\
\text { ist eine effektive Art } \\
\text { der Erhaltung der } \\
\text { biologischen Vielfalt. } \\
\\
\text { Vorschlagen von } \\
\text { verboten, strengem } \\
\text { Natursschutz. }\end{array}$ \\
\hline
\end{tabular}




\section{Block IV： Inter- und intragenerationale Gerechtigkeit}

\begin{tabular}{|l|l|l|l|}
\hline \multicolumn{1}{|c|}{$\begin{array}{c}\text { Forschungs- } \\
\text { interesse }\end{array}$} & \multicolumn{1}{|c|}{$\begin{array}{c}\text { Frage- } \\
\text { interventionen }\end{array}$} & \multicolumn{1}{c|}{ Intervención } & Erwartungshorizont \\
\hline $\begin{array}{l}\text { Sehen SuS } \\
\text { Gerechtigkeitskompo } \\
\text { nenten zwischen } \\
\text { heutigen und } \\
\text { künftigen } \\
\text { Generationen? }\end{array}$ & $\begin{array}{l}\text { 14. Welche Rolle spielt } \\
\text { die ungleiche } \\
\text { Verteilung von } \\
\text { Reichtum für den } \\
\text { Schutz der } \\
\text { Biologischen Vielfalt? }\end{array}$ & $\begin{array}{l}\text { 14. ¿Qué } \\
\text { influencia tiene la } \\
\text { distribución } \\
\text { desigual de riqueza } \\
\text { y pobreza para la } \\
\text { protección de la } \\
\text { variedad } \\
\text { biologica? }\end{array}$ & $\begin{array}{l}\text { Bewahrung der } \\
\text { biologischen Vielfalt } \\
\text { muss für künftige } \\
\text { Generationen } \\
\text { erfolgen. }\end{array}$ \\
& $\begin{array}{l}\text { 14.a Haben } \\
\text { zukünftige } \\
\text { Generationen beim } \\
\text { Schutz von } \\
\text { Biologischer Vielfalt } \\
\text { für dich eine } \\
\text { Bedeutung? }\end{array}$ & $\begin{array}{l}\text { 14.a ¿Tienen las } \\
\text { generaciónes } \\
\text { siguientes una } \\
\text { importancia para ti } \\
\text { por la protección } \\
\text { de la variedad } \\
\text { biologica? }\end{array}$ & $\begin{array}{l}\text { Durch Armut entsteht } \\
\text { Druck auf natürliche } \\
\text { Ressourcen und somit } \\
\text { auf die Biodiversität }\end{array}$ \\
\hline
\end{tabular}

\section{Block V: Nachfragen}

\begin{tabular}{|c|c|c|c|}
\hline $\begin{array}{l}\text { Forschungs- } \\
\text { interesse }\end{array}$ & $\begin{array}{l}\text { Frage- } \\
\text { interventionen }\end{array}$ & Intervención & Erwartungshorizont \\
\hline Quellen & $\begin{array}{l}\text { 15. Ich war überrascht, } \\
\text { dass du so viel zu den } \\
\text { einzelnen Fragen } \\
\text { gesagt hast. } \\
\text { Woher weißt du } \\
\text { darüber so viel? } \\
\text { Auf welche Quellen } \\
\text { hast du } \\
\text { zurückgegriffen? } \\
\text { Habt Ihr die } \\
\text { Biodiversität oder } \\
\text { damit } \\
\text { zusammenhängende } \\
\text { Probleme schon einmal } \\
\text { in der Schule } \\
\text { behandelt? }\end{array}$ & $\begin{array}{l}15 . \quad \text { Fue una } \\
\text { agradable sorpresa } \\
\text { para mi que sabiste } \\
\text { tan mucho. } \\
\text { ¿De dónde tienes } \\
\text { tus informaciónes? } \\
\text { ¿Qué fuentes } \\
\text { utilisaste? } \\
\text { ¿Era la variedad } \\
\text { biologica o los } \\
\text { problemas } \\
\text { relacionadas un } \\
\text { tema en la escuela? }\end{array}$ & $\begin{array}{l}\text { Schule (Biologie, } \\
\text { Erdkunde, Werte und } \\
\text { Normen), } \\
\text { Fernsehen, } \\
\text { Filme (The Day After } \\
\text { Tomorrow), } \\
\text { eigenes Interesse, } \\
\text { Urlaubserfahrungen, } \\
\text { Internet }\end{array}$ \\
\hline
\end{tabular}




\begin{tabular}{|l|l|l|l|}
\hline \multicolumn{1}{|c|}{$\begin{array}{c}\text { Forschungs- } \\
\text { interesse }\end{array}$} & \multicolumn{1}{|c|}{$\begin{array}{c}\text { Frage- } \\
\text { interventionen }\end{array}$} & \multicolumn{1}{c|}{ Intervención } & Erwartungshorizont \\
\hline $\begin{array}{l}\text { Gab es } \\
\text { Schwierigkeiten mit } \\
\text { dem Leitfaden oder } \\
\text { können kognitive } \\
\text { Konflikte explizit } \\
\text { genannt werden? }\end{array}$ & $\begin{array}{l}\text { 15.a Was war für dich } \\
\text { während des } \\
\text { Interviews schwierig? }\end{array}$ & $\begin{array}{l}\text { 15.a ¿Qué fui } \\
\text { difícilpara ti en el } \\
\text { entrevista? }\end{array}$ & $\begin{array}{l}\text { Unsicherheit, ob } \\
\text { geäußert wird, was } \\
\text { wirklich gefragt war; } \\
\text { Wiederholungen }\end{array}$ \\
\hline $\begin{array}{l}\text { Können von den } \\
\text { interviewten SuS } \\
\text { selbst bereits } \\
\text { Lerneffekte genannt } \\
\text { werden? }\end{array}$ & $\begin{array}{l}\text { 15.b Was waren neue } \\
\text { Gedanken? }\end{array}$ & $\begin{array}{l}\text { 15.b ¿Qué } \\
\text { pensadas nuevas } \\
\text { tuviste? }\end{array}$ & $\begin{array}{l}\text { - Keine; } \\
\text { - Biologisches, } \\
\text { Wirtschaftliches, } \\
\text { Politisches hängt } \\
\text { alles zusammen; } \\
\text {-Forschungsinteresse } \\
\text { geweckt (neugierig, } \\
\text { ob Antworten } \\
\text { richtig), } \\
\text {-aus Vorwissen alles } \\
\text { logisch erschlossen }\end{array}$ \\
\hline
\end{tabular}




\section{Kurzfragebogen}

1. Schülerin oder Schüler der Schule:

2. Klassenstufe:

3. Alter:

4. Wohnort:

5. Geschlecht:

6. Mitgliedschaft in Umwelt- / Naturschutzorganisationen oder Teilnahme an Umwelt- / Naturschutzprojekten: ja:........ nein:........

6.1. Wenn ja, Name der Organisation(en) bzw. des(r) Projekte(s) und der Aktivitäten, an denen teilgenommen wird /wurde:

Wie lange bist Du in dieser Gruppe aktiv?

\section{Cuestionario}

1. Escuela

3. Clase:

4. Edad:

5. ¿Dónde creciste? ¿En la ciudad o en el campo?

6. Sexo:

7. ¿Eres membre en una organsación o gupo de la naturaleza ? ¿Participaste en algunos proyetos para la protección de la naturaleza?

8. ¿Qué más quieres que decirnos? 


\section{Appendix B}

Item keys for the German and Spanish questionnaire. 


\section{Questionnaire Part I}

Table 13.1. Items of the questionnaire part I

\begin{tabular}{|c|c|c|c|}
\hline Scale & Item context & $\begin{array}{l}\text { Questionnaire page } \\
\text { number, } \\
\text { (item number) }\end{array}$ & $\begin{array}{l}\text { Total of } \\
\text { finally } \\
\text { included } \\
\text { items }\end{array}$ \\
\hline Terms & $\begin{array}{l}1 \text { Item biodiversity } \\
1 \text { Item biological diversity }\end{array}$ & $2 \mathrm{I},(1-2)$ & 2 \\
\hline \multirow{4}{*}{ Definition } & 4 Items incorrect biological diversity & $2 \mathrm{II}(3,4,6,7)$ & \multirow{4}{*}{11} \\
\hline & 4 Items correct biological diversity & 2 II $(1,2,5,8)$ & \\
\hline & 2 Items correct biodiversity & $3(10,11)$ & \\
\hline & 2 Items incorrect biodiversity & $2(9 *) ; 3(12)$ & \\
\hline \multirow{2}{*}{$\begin{array}{l}\text { Distribution } \\
\text { of } \\
\text { biodiversity }\end{array}$} & 4 Items correct & $3\left(2,3,5^{*}, 7\right)$ & \multirow{2}{*}{5} \\
\hline & 4 Items incorrect & $3\left(1,4^{*}, 6^{*}, 8\right)$ & \\
\hline \multirow{6}{*}{$\begin{array}{l}\text { Threats to } \\
\text { biodiversity }\end{array}$} & 3 Items correct ecology & $4\left(6,10^{*}\right) ; 5\left(17^{*}\right)$ & \multirow{6}{*}{9} \\
\hline & 3 Items incorrect ecology & $4(4,14) ; 5\left(18^{*}\right)$ & \\
\hline & 3 Items correct economy & $4\left(1^{*}, 3,9\right)$ & \\
\hline & 3 Items incorrect economy & $4\left(4,7^{*}, 12^{*}\right)$ & \\
\hline & 3 Items correct social issues & $4\left(2,13^{*}\right) ; 5(15)$ & \\
\hline & 3 Items incorrect social issues & $4\left(5,8^{*}, 11^{*}\right)$ & \\
\hline
\end{tabular}

* Item was not analysed within the scope of the dissertation

\section{Questionnaire Part II}

Table 13.2. Items of the questionnaire part II

\begin{tabular}{|l|l|l|l|l|}
\hline & Subscale & Chosen Context & $\begin{array}{l}\text { Questionnaire } \\
\text { page number, } \\
\text { (item number) }\end{array}$ & $\begin{array}{l}\text { Total of } \\
\text { finally } \\
\text { included } \\
\text { items }\end{array}$ \\
\hline $\begin{array}{l}\text { Awareness } \\
\text { of } \\
\text { consequenc } \\
\text { es } \\
\text { (AC) }\end{array}$ & \multirow{2}{*}{$\begin{array}{l}\text { Egoistic } \\
\text { (ACego) }\end{array}$} & 1 Item species & $3(2)$ & \multirow{2}{*}{5} \\
\cline { 3 - 4 } & 1 Item protection & $3(11)$ & \\
\cline { 3 - 4 } & 3 Items biological diversity & $3\left(1,6 *, 9^{*}\right)$ & \\
\hline
\end{tabular}


Table 13.2 (continued)

\begin{tabular}{|c|c|c|c|c|}
\hline \multirow{6}{*}{$\begin{array}{l}\text { Awareness of } \\
\text { Consequences } \\
\text { (AC) (cont.) }\end{array}$} & \multirow{3}{*}{$\begin{array}{l}\text { Altruistic } \\
\text { (ACalt) }\end{array}$} & 1 Item species & $4\left(13^{*}\right)$ & \multirow{3}{*}{5} \\
\hline & & 1 Item protection & $4\left(15^{*}\right)$ & \\
\hline & & $\begin{array}{l}3 \text { Items biological } \\
\text { diversity }\end{array}$ & $3(5,7,8)$ & \\
\hline & \multirow{3}{*}{$\begin{array}{l}\text { Biospheric } \\
\text { (ACbio) }\end{array}$} & 1 Item species & $4(12 *)$ & \multirow{3}{*}{5} \\
\hline & & 1 Item protection & $3\left(4^{*}\right)$ & \\
\hline & & $\begin{array}{l}3 \text { Items biological } \\
\text { diversity }\end{array}$ & $3(3,10) ; 4(14)$ & \\
\hline \multirow{4}{*}{$\begin{array}{l}\text { Problem } \\
\text { perception } \\
(\mathrm{PW})\end{array}$} & $\begin{array}{l}\text { ecological } \\
\text { global/local } \\
\text { (PWoel) }\end{array}$ & Miscellaneous & $\begin{array}{l}7(2,4) ; 8 \text { (8); } 9 \\
(11)\end{array}$ & $4(8)$ \\
\hline & $\begin{array}{l}\text { economic } \\
\text { global/local } \\
\text { (PWoen) }\end{array}$ & Miscellaneous & $7(1) ; 8(6,7,9)$ & $4(8)$ \\
\hline & \begin{tabular}{|l} 
social \\
global/local \\
(PWsoz)
\end{tabular} & Miscellaneous & $\begin{array}{l}7(3,5) ; 8(19) ; 9 \\
(12)\end{array}$ & $4(8)$ \\
\hline & $\begin{array}{l}\text { Problem denial } \\
\text { global/local } \\
\text { (PWnei) }\end{array}$ & Miscellaneous & $10(13-16)$ & $4(8)$ \\
\hline \multirow{8}{*}{$\begin{array}{l}\text { Perceived } \\
\text { ability to } \\
\text { reduce threat } \\
\& \\
\text { ascription of } \\
\text { responsibility }\end{array}$} & \multirow{4}{*}{$\begin{array}{l}\text { Perceived } \\
\text { ability to reduce } \\
\text { threats (PA) }\end{array}$} & 1 Item medical plants & $12(5)$ & \multirow{4}{*}{$4(8)$} \\
\hline & & 1 Item cosmetics & $12(6)$ & \\
\hline & & 1 Item species & $12(8)$ & \\
\hline & & 1 Item biological diversity & $17(7)$ & \\
\hline & \multirow{4}{*}{$\begin{array}{l}\text { Ascription of } \\
\text { Responsibility } \\
\text { (AR) }\end{array}$} & 1 Item medical plants & $11(1)$ & \multirow{4}{*}{$4(8)$} \\
\hline & & 1 Item cosmetics & $11(2)$ & \\
\hline & & 1 Item species & $11(4)$ & \\
\hline & & 1 Item biological diversity & $11(3)$ & \\
\hline NEP & NEP & $\begin{array}{l}15 \text { original items after } \\
\text { Dunlap et al. (2000) }\end{array}$ & $13(1-15)$ & 15 \\
\hline \multirow{4}{*}{$\begin{array}{l}\text { Personal } \\
\text { Norms } \\
(\mathrm{PN})\end{array}$} & \multirow{4}{*}{$\begin{array}{l}\text { Global /local } \\
\text { personal norms } \\
\text { (PN) }\end{array}$} & 1 Item medical plants & $14(2)$ & \multirow{4}{*}{4} \\
\hline & & 1 Item cosmetics & $14(3)$ & \\
\hline & & 1 Item species & $14(4)$ & \\
\hline & & 1 Item biological diversity & $14(1)$ & \\
\hline
\end{tabular}


Table 13.2 (continued)

\begin{tabular}{|c|c|c|c|c|}
\hline \multirow{4}{*}{$\begin{array}{l}\text { Behavioural } \\
\text { Commitment } \\
(\mathrm{HN})\end{array}$} & \multirow{4}{*}{$\begin{array}{l}\text { Activism } \\
\text { (Hnact) }\end{array}$} & 1 Item medical plants & $15(9)$ & \multirow{4}{*}{4} \\
\hline & & 1 Item cosmetics & $15(7)$ & \\
\hline & & 1 Item species & $15(4)$ & \\
\hline & & 1 Item biological diversity & $16(14)$ & \\
\hline & \multirow{4}{*}{$\begin{array}{l}\text { Nonactivist } \\
\text { Public-Sphere } \\
\text { behaviour } \\
\text { (HNnps) }\end{array}$} & 1 Item medical plants & $16(13)$ & \multirow{4}{*}{4} \\
\hline & & 1 Item cosmetics & $15(6)$ & \\
\hline & & 1 Item species & $15(8)$ & \\
\hline & & 1 Item biological diversity & $15(1)$ & \\
\hline & \multirow{4}{*}{$\begin{array}{l}\text { Private-Shpere } \\
\text { Behaviour } \\
\text { (HNps) }\end{array}$} & 1 Item medical plants & $15(10)$ & \multirow{4}{*}{4} \\
\hline & & 1 Item cosmetics & $15(3)$ & \\
\hline & & 1 Item species & $15(2)$ & \\
\hline & & 1 Item biological diversity & $16(12)$ & \\
\hline & $\begin{array}{l}\text { Behaviour in } \\
\text { Organisations* } \\
\text { (Hnorg) }\end{array}$ & $\begin{array}{l}2 \text { Items involvement at } \\
\text { school }\end{array}$ & $15(5,11)$ & 2 \\
\hline \multirow{10}{*}{$\begin{array}{l}\text { Schwartz } \\
\text { Values }\end{array}$} & Vacon & Conformity* & $\begin{array}{l}17 \text { (7); } 18 \text { (16); } 19 \\
\text { (28); } 20 \text { (36) }\end{array}$ & 4 \\
\hline & Vatrad & Tradition & $\begin{array}{l}18(9) ; 19(20,25) ; \\
20(38)\end{array}$ & 4 \\
\hline & Vabene & Benevolence & $\begin{array}{l}18(12,18) ; 19(27) ; \\
20(33)\end{array}$ & 4 \\
\hline & Vauni & Universalism & $\begin{array}{l}17(3,8) ; 18(19) ; \\
19(23,29) ; 20(40)\end{array}$ & 6 \\
\hline & Vased & Self-Direction & $\begin{array}{l}17 \text { (1); } 18(11) ; 19 \\
(22) ; 20(34)\end{array}$ & 4 \\
\hline & Vasti & Stimulation & $\begin{array}{l}17 \text { (6); } 18 \text { (15); } 20 \\
(30)\end{array}$ & 3 \\
\hline & Vahed & Hedonism* & $\begin{array}{l}18 \text { (10); } 19 \text { (26); } 20 \\
(37)\end{array}$ & 3 \\
\hline & Vaach & Achievement & $\begin{array}{l}17 \text { (4); } 18 \text { (13); } 19 \\
\text { (24); } 20 \text { (32) }\end{array}$ & 4 \\
\hline & VApow & Power & $\begin{array}{l}17 \text { (2); } 18 \text { (17); } 20 \\
(39)\end{array}$ & 3 \\
\hline & VAsec & Security & $\begin{array}{l}17(5) ; 18(14) ; 19 \\
(21), 20(31,35)\end{array}$ & 5 \\
\hline
\end{tabular}

\footnotetext{
* Item or Scale was not analysed within the scope of the dissertation
} 
Appendix C

German questionnaire of the quantitative study.

The Spanish version can be retrieved from the author upon request.

Please note: Original format: DIN A 5 booklets. 


\section{Fragebogen zu Alltagswissen über lokale und globale Biodiversität}

\section{Pilotstudie 2005 - Teil I \\ Susanne Menzel \& Susanne Bögeholz}

\section{Liebe Schülerin, lieber Schüler,}

vielen Dank für Ihre Bereitschaft, diesen Fragebogen auszufüllen! Ihre Antworten können dazu beitragen, dass wir besser verstehen können, was Schüler(innen) über das Thema biologische Vielfalt wissen und denken. Dies kann helfen, Biologieunterricht besser zu gestalten. Bitte arbeiten Sie dieses Heftchen sorgfältig von vorn bis hinten durch.

\section{Das Fragebogen Team}

Zunächst möchten wir Sie bitten, folgende Angaben zu machen:

$\Rightarrow$ Die ersten beiden Buchstaben des Vornamens Ihrer Mutter:

$\Rightarrow$ Ihr Geburtstag (nur der Tag; z.B. 8. Juni =08)

$\Rightarrow$ Die ersten beiden Buchstaben des Vornamens Ihres Vaters:
Alter: Jahre
Geschlecht:
weiblich
männlich

Für Antworten gibt es meistens die Möglichkeit, zwischen "Stimme zu" und "Stimme nicht zu" auszuwählen. Bitte beachten Sie, dass immer nur eine Antwortmöglichkeit angekreuzt werden darf.

\section{Beispiel:}

\begin{tabular}{|l|c|c|}
\hline & Stimme zu & $\begin{array}{c}\text { Stimme } \\
\text { nicht zu }\end{array}$ \\
\hline Ich kenne sehr viele heimische Tier- und Pflanzenarten. & $\square$ & \\
\hline
\end{tabular}

Viel Spaß beim Ausfüllen!

\footnotetext{
${ }^{1}$ Georg-August-Universität Göttingen, Abteilung Didaktik der Biologie, Biologische Fakultät, Waldweg 26, 37073 Göttingen. Kontakt: Susanne Menzel, susanne.menzel@gwdg.de, Tel. 0551-39-14015 
I. Bitte beantworten Sie zunächst die folgenden beiden Fragen:

\begin{tabular}{|l|c|c|c|c|}
\hline & $\begin{array}{c}\text { mehr als } \\
\text { fünf mal }\end{array}$ & $\begin{array}{c}\text { drei bis } \\
\text { fünf mal }\end{array}$ & $\begin{array}{c}\text { ein- bis } \\
\text { zweimal }\end{array}$ & $\begin{array}{c}\text { noch } \\
\text { nie }\end{array}$ \\
\hline $\begin{array}{l}\text { 1) Wie häufig haben Sie den Begriff } \\
\text { Biodiversität schon gehört? }\end{array}$ & $\square$ & $\square$ & $\square$ \\
\hline $\begin{array}{l}\text { 2) Wie oft haben Sie den Begriff } \\
\text { biologische Vielfalt schon gehört? }\end{array}$ & $\square$ & $\square$ & $\square$ \\
\hline
\end{tabular}

II. Nun möchten wir Ihnen einige ganz allgemeine Fragen zu den Begriffen "biologische Vielfalt" und "Biodiversität" stellen. Es ist überhaupt nicht schlimm, wenn Sie diese Begriffe nicht kennen. Am hilfreichsten ist es für uns, wenn Sie ganz ehrlich antworten.

\begin{tabular}{|l|c|c|}
\hline & $\begin{array}{c}\text { Stimme } \\
\text { zu }\end{array}$ & $\begin{array}{c}\text { Stimme } \\
\text { nicht zu }\end{array}$ \\
\hline $\begin{array}{l}\text { 1) Unter biologischer Vielfalt versteht man ganz allgemein } \\
\text { „die Vielfalt des Lebens". }\end{array}$ & $\square$ & $\square$ \\
\hline $\begin{array}{l}\text { 2) Die biologische Vielfalt umfasst unter anderem den } \\
\text { Menschen. }\end{array}$ & $\square$ & $\square$ \\
\hline $\begin{array}{l}\text { 3) Biologische Vielfalt bezeichnet die verschiedenen } \\
\text { biologischen Themen. }\end{array}$ & $\square$ & $\square$ \\
\hline 4) Viren zählen nicht zur biologischen Vielfalt. & $\square$ & $\square$ \\
\hline $\begin{array}{l}\text { 5) Die biologische Vielfalt kann man in die Vielfalt der Gene, } \\
\text { der Arten und der Ökosysteme unterteilen. }\end{array}$ & $\square$ & $\square$ \\
\hline $\begin{array}{l}\text { 6) Biologische Vielfalt ist gleichzusetzen mit der Vielfalt } \\
\text { von Tier- und Pflanzenarten. }\end{array}$ & $\square$ & $\square$ \\
\hline $\begin{array}{l}\text { 7) Die biologische Vielfalt bezeichnet die vielen } \\
\text { verschiedenen Ideen in der Wissenschaft Biologie. }\end{array}$ & $\square$ & $\square$ \\
\hline 8) Mikroorganismen zählen auch zur biologischen Vielfalt. & $\square$ & $\square$ \\
\hline $\begin{array}{l}\text { 9) Biodiversität beschreibt die Vielfalt von Fachrichtungen } \\
\text { innerhalb der Biologie. }\end{array}$ & $\square$ & $\square$ \\
\hline
\end{tabular}




\begin{tabular}{|l|c|c|}
\hline & $\begin{array}{c}\text { Stimme } \\
\text { zu }\end{array}$ & $\begin{array}{c}\text { Stimme } \\
\text { nicht zu }\end{array}$ \\
\hline $\begin{array}{l}\text { 10) Die Biodiversität kann man in die Vielfalt der Gene, der } \\
\text { Arten und der Ökosysteme unterteilen. }\end{array}$ & $\square$ & $\square$ \\
\hline 11) Biodiversität ist das Fremdwort für biologische Vielfalt. & $\square$ & $\square$ \\
\hline $\begin{array}{l}\text { 12) Biodiversität ist eine Forschungsmethode in der } \\
\text { Biologie. }\end{array}$ & $\square$ & $\square$ \\
\hline
\end{tabular}

III. Im Folgenden finden Sie einige Aussagen zum Vorkommen der biologischen Vielfalt. Stimmen Sie zu?

\begin{tabular}{|l|c|c|}
\hline & $\begin{array}{c}\text { Stimme } \\
\text { zu }\end{array}$ & $\begin{array}{c}\text { Stimme } \\
\text { nicht zu }\end{array}$ \\
\hline $\begin{array}{l}\text { 1) Die Regionen mit besonders hoher biologischer Vielfalt } \\
\text { liegen vor allem in Europa. }\end{array}$ & $\square$ & $\square$ \\
\hline $\begin{array}{l}\text { 2) In den Regionen um den Äquator ist die biologische } \\
\text { Vielfalt besonders groß. }\end{array}$ & $\square$ & $\square$ \\
\hline $\begin{array}{l}\text { 3) Es gibt Pflanzen, die auf der ganzen Welt heimisch sind, } \\
\text { während andere nur an bestimmten Orten vorkommen. }\end{array}$ & $\square$ & $\square$ \\
\hline $\begin{array}{l}\text { 4) Ob die biologische Vielfalt an einem Ort hoch oder } \\
\text { niedrig ist, hängt vor allem vom Klima ab. }\end{array}$ & $\square$ & $\square$ \\
\hline $\begin{array}{l}\text { 5) Dort, wo besonders wenige Nährstoffe im Boden } \\
\text { vorkommen, ist die biologische Vielfalt besonders hoch. }\end{array}$ & $\square$ & $\square$ \\
\hline $\begin{array}{l}\text { 6) Dort, wo Menschen leben, ist die biologische Vielfalt } \\
\text { grundsätzlich niedriger. }\end{array}$ & $\square$ & $\square$ \\
\hline $\begin{array}{l}\text { 7) Tropische Regenwälder sind Orte mit besonders hoher } \\
\text { biologischer Vielfalt. }\end{array}$ & $\square$ & $\square$ \\
\hline $\begin{array}{l}\text { 8) Industrieländer verfügen über eine besonders hohe } \\
\text { biologische Vielfalt. }\end{array}$ & $\square$ & $\square$ \\
\hline
\end{tabular}


IV. Nun kommen wir zu einigen Aussagen darüber, ob und wenn ja, wodurch die biologische Vielfalt gefährdet sein könnte.

Bitte kreuzen Sie auch hier an, ob Sie zustimmen oder nicht.

\begin{tabular}{|c|c|c|}
\hline Die biologische Vielfalt ist gefährdet & $\begin{array}{l}\text { Stimme } \\
\mathrm{zu}\end{array}$ & $\begin{array}{l}\text { Stimme } \\
\text { nicht zu }\end{array}$ \\
\hline $\begin{array}{l}\text { 1)...weil es zu wenige Unternehmen gibt, die } \\
\text { umweltschonend produzieren. }\end{array}$ & $\square$ & $\square$ \\
\hline $\begin{array}{l}\text { 2)...weil die Armut von Menschen aus Entwicklungsländern } \\
\text { dazu führt, dass sie natürliche Ressourcen übernutzen } \\
\text { müssen. }\end{array}$ & $\square$ & $\square$ \\
\hline $\begin{array}{l}\text { 3)... weil mit seltenen Tieren und Pflanzen Geschäfte } \\
\text { gemacht werden. }\end{array}$ & $\square$ & $\square$ \\
\hline $\begin{array}{l}\text { 4)...weil manche Pflanzen und Tiere nicht so gut an ihre } \\
\text { Umgebung angepasst sind. }\end{array}$ & $\square$ & $\square$ \\
\hline $\begin{array}{l}\text { 5)...weil die meisten Menschen in den Industriestaaten } \\
\text { nicht genug Geld haben für Produkte, die die biologische } \\
\text { Vielfalt schonen würden. }\end{array}$ & $\square$ & $\square$ \\
\hline $\begin{array}{l}\text { 6)...weil die Gebiete, in denen Arten ungestört leben } \\
\text { können, kleiner und weniger werden. }\end{array}$ & $\square$ & $\square$ \\
\hline $\begin{array}{l}\text { 7)...weil Unternehmen keinen Einfluss darauf haben, ob sie } \\
\text { umweltschonend produzieren oder nicht. }\end{array}$ & $\square$ & $\square$ \\
\hline $\begin{array}{l}\text { 8)...weil viele Menschen nicht verstehen, was sie anrichten, } \\
\text { wenn sie bedrohte Tiere oder Pflanzen verkaufen. }\end{array}$ & $\square$ & $\square$ \\
\hline $\begin{array}{l}\text { 9)...weil viele Güter aus bedrohten Pflanzen und Tieren } \\
\text { begehrt sind. }\end{array}$ & $\square$ & $\square$ \\
\hline $\begin{array}{l}\text { 10)...weil Pflanzen, die ursprünglich nicht in einem Gebiet } \\
\text { vorkommen, andere Pflanzen verdrängen. }\end{array}$ & $\square$ & $\square$ \\
\hline $\begin{array}{l}\text { 11)...weil der Mensch auf der Erde keinen Platz für } \\
\text { biologische Vielfalt lässt. }\end{array}$ & $\square$ & $\square$ \\
\hline $\begin{array}{l}\text { 12)...weil Wirtschaftsunternehmen immer auch seltene } \\
\text { Pflanzen verkaufen müssen. }\end{array}$ & $\square$ & $\square$ \\
\hline $\begin{array}{l}\text { 13) ... weil soziale Gründe eine große Rolle bei der Zerstörung } \\
\text { der biologischen Vielfalt spielen. }\end{array}$ & $\square$ & $\square$ \\
\hline
\end{tabular}




\begin{tabular}{|l|c|c|}
\hline Die biologische Vielfalt ist gefährdet & $\begin{array}{c}\text { Stimme } \\
\text { zu }\end{array}$ & $\begin{array}{c}\text { Stimme } \\
\text { nicht zu }\end{array}$ \\
\hline $\begin{array}{l}\text { 14)...weil schwierige ökologische Bedingungen in manchen } \\
\text { Regionen herrschen (z.B. Trockenheit in der Wüste, Kälte an } \\
\text { den Polen). }\end{array}$ & $\square$ & $\square$ \\
\hline $\begin{array}{l}\text { 15)...weil Käufer nicht genug über Produkte wissen, die } \\
\text { möglicherweise die biologische Vielfalt bedrohen. }\end{array}$ & $\square$ & $\square$ \\
\hline $\begin{array}{l}16 \text {...weil sich Wirtschaft und Handel zwar korrekt } \\
\text { verhalten, andere Bedingungen aber zu ungünstig sind. }\end{array}$ & $\square$ & $\square$ \\
\hline $\begin{array}{l}\text { 17)...weil Naturkatastrophen wie Vulkanausbrüche, Feuer } \\
\text { oder Überschwemmungen Tier- und Pflanzenarten } \\
\text { vernichten können. }\end{array}$ & $\square$ & $\square$ \\
\hline $\begin{array}{l}\text { 18)...weil manche Pflanzen auf der Welt zu langsam } \\
\text { wachsen. }\end{array}$ & $\square$ & $\square$ \\
\hline
\end{tabular}




\section{Vielen Dank!}

Wir möchten Sie nun bitten, sich ein persönliches Codewort auszudenken, dass Sie gut behalten können. Es spielt keine Rolle, wie lang das Codewort ist oder was es bedeutet.

Sie werden in Teil II des Fragebogens aufgefordert, dieses Codewort nochmals einzutragen.

Mein persönliches Codewort lautet:

Schon einmal vielen Dank für das Ausfüllen!

Und bis gleich!

(;) 


\section{Fragebogen zur}

\section{Wahrnehmung lokaler und globaler Biodiversität}

\section{Susanne Menzel \& Susanne Bögeholz}

\section{Liebe Schülerin, lieber Schüler!}

Damit wir Ihre Daten dem ersten Teil des Fragebogens zuordnen können, bitten wir Sie erneut um die Angabe Ihrer Codes.

$\Rightarrow$ Die ersten beiden Buchstaben des Vornamens Ihrer Mutter:

$\Rightarrow$ Ihr Geburtstag (nur der Tag; z.B. 8. Juni = 08):

$\Rightarrow$ Die ersten beiden Buchstaben des Vornamens Ihres Vaters:

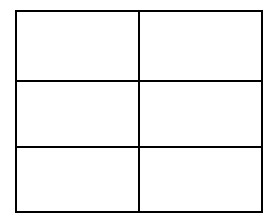

Ihr persönliches Codewort:

In diesem Fragebogen gibt es neben den Fragen zwei Informationstexte. Lesen Sie die Texte sorgfältig durch und bearbeiten Sie den Fragebogen der Reihenfolge nach.

Es gibt für jede Frage mehrere mögliche Antworten. Bitte beachten Sie, dass hier immer nur eine Antwortmöglichkeit pro Frage angekreuzt werden darf.

Beispiel:

\begin{tabular}{|lcccc|}
\hline & Stimme zu & $\begin{array}{c}\text { Stimme } \\
\text { weitgehend } \\
\text { zu }\end{array}$ & $\begin{array}{c}\text { Stimme } \\
\text { teilweise } \\
\text { zu }\end{array}$ & $\begin{array}{c}\text { Stimme } \\
\text { nicht zu }\end{array}$ \\
\hline $\begin{array}{l}\text { Ich kenne sehr viele heimische Tier- und } \\
\text { Pflanzenarten. }\end{array}$ & $\square$ & $\square$ & $\square$ & $\square$ \\
\hline
\end{tabular}

Viel Spaß beim Ausfüllen!

\footnotetext{
${ }^{1}$ Georg-August-Universität Göttingen, Abteilung Didaktik der Biologie, Biologische Fakultät, Waldweg 26, 37073 Göttingen. Kontakt: Susanne Menzel, susanne.menzel@gwdg.de, Tel. 0551-39-14015
} 
Die Begriffe biologische Vielfalt und Biodiversität sind bedeutungsgleich. Ganz allgemein beschreiben die beiden Begriffe "die Vielfalt des Lebens". Darunter versteht man nicht nur beispielsweise verschiedene Tiere, Pflanzen, Pilze oder Mikroorganismen, sondern auch die

- Vielfalt von Ökosystemen,

- die Artenvielfalt und

- die genetische Vielfalt.

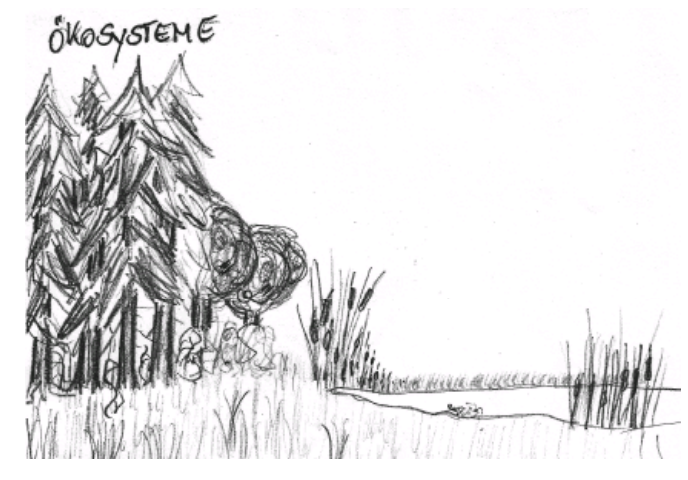

Die Vielfalt von Ökosystemen beschreibt die vielen verschiedenen Ökosysteme, die es auf der Erde gibt. Beispiele für Ökosysteme sind der See, der Wald oder die Wiese.

Die Artenvielfalt ist ebenfalls ein wichtiges Element der Biodiversität oder biologischen Vielfalt. Sie beschreibt die Arten, die auf dieser Erde existieren. Viele dieser Arten (z.B. viele Insektenarten) sind noch unentdeckt.
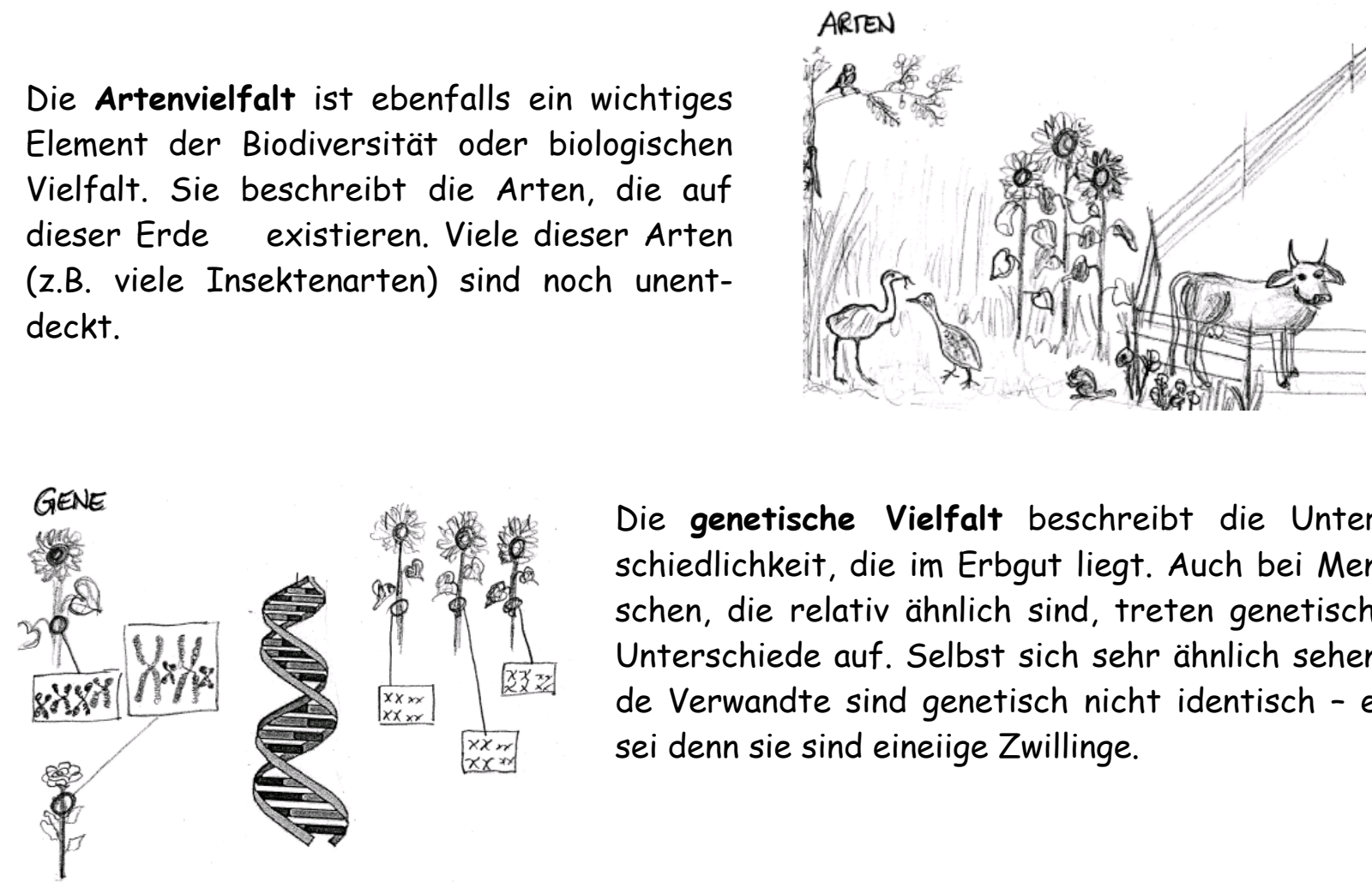

Die genetische Vielfalt beschreibt die Unterschiedlichkeit, die im Erbgut liegt. Auch bei Menschen, die relativ ähnlich sind, treten genetische Unterschiede auf. Selbst sich sehr ähnlich sehende Verwandte sind genetisch nicht identisch - es sei denn sie sind eineiige Zwillinge.

Die biologische Vielfalt, also die Vielfalt der Ökosysteme, der Arten und die genetische Vielfalt, geht stark zurück. Zum Schutz der Biodiversität sind in den letzten Jahren sowohl national wie auch international eine Reihe von Gesetzen verabschiedet worden. 
I. Hier sind einige Aussagen zu den Folgen des Verlusts der biologischen Vielfalt aufgeführt. Bitte kreuzen Sie an, inwieweit Sie den einzelnen Aussagen zustimmen.

\begin{tabular}{|c|c|c|c|c|}
\hline & $\begin{array}{c}\text { Stimme } \\
\mathrm{zu}\end{array}$ & $\begin{array}{c}\text { Stimme } \\
\text { weitgehend } \\
\text { zu }\end{array}$ & $\begin{array}{l}\text { Stimme } \\
\text { teilweise } \\
\text { zu }\end{array}$ & $\begin{array}{c}\text { Stimme } \\
\text { nicht } \\
\text { zu }\end{array}$ \\
\hline $\begin{array}{l}\text { 1. Der Verlust der biologischen Vielfalt } \\
\text { wird für mich und meine Familie ernsthafte } \\
\text { Konsequenzen haben. }\end{array}$ & $\square$ & $\square$ & $\square$ & $\square$ \\
\hline $\begin{array}{l}\text { 2. Durch das fortschreitende Artenster- } \\
\text { ben kann ich weniger Naturprodukte kau- } \\
\text { fen. }\end{array}$ & $\square$ & $\square$ & $\square$ & $\square$ \\
\hline $\begin{array}{l}\text { 3. Der Verlust der biologischen Vielfalt } \\
\text { wird vor allem die Natur selbst durchein- } \\
\text { ander bringen. }\end{array}$ & $\square$ & $\square$ & $\square$ & $\square$ \\
\hline $\begin{array}{l}\text { 4. Der Schutz der biologischen Vielfalt } \\
\text { nutzt vor allem der Natur selbst. }\end{array}$ & $\square$ & $\square$ & $\square$ & $\square$ \\
\hline $\begin{array}{l}\text { 5. Der Verlust der biologischen Vielfalt } \\
\text { wird ein ernsthaftes Problem für die Ge- } \\
\text { sellschaft sein. }\end{array}$ & $\square$ & $\square$ & $\square$ & $\square$ \\
\hline $\begin{array}{l}\text { 6. Eine Natur mit hoher biologischer Viel- } \\
\text { falt dient dazu, dass ich mich daran er- } \\
\text { freuen und darin erholen kann. }\end{array}$ & $\square$ & $\square$ & $\square$ & $\square$ \\
\hline $\begin{array}{l}\text { 7. Der Verlust der biologischen Vielfalt } \\
\text { wird allen Menschen schaden, da so medizi- } \\
\text { nische Wirkstoffe aus noch unentdeckten } \\
\text { Arten verloren gehen. }\end{array}$ & $\square$ & $\square$ & $\square$ & $\square$ \\
\hline $\begin{array}{l}\text { 8. Leidtragende unter dem Verlust der bio- } \\
\text { logischen Vielfalt sind vor allem auch die } \\
\text { Menschen künftiger Generationen. }\end{array}$ & $\square$ & $\square$ & $\square$ & $\square$ \\
\hline $\begin{array}{l}\text { 9. Der Verlust gewisser Arten wäre nicht } \\
\text { so tragisch, da sie mich dann im Alltag } \\
\text { nicht mehr stören. }\end{array}$ & $\square$ & $\square$ & $\square$ & $\square$ \\
\hline $\begin{array}{l}\text { 10. Der Verlust von biologischer Vielfalt } \\
\text { wird ernsthafte Konsequenzen für alle Tie- } \\
\text { re und Pflanzen haben. }\end{array}$ & $\square$ & $\square$ & $\square$ & $\square$ \\
\hline $\begin{array}{l}\text { 11. Verstärkter Schutz der biologischen } \\
\text { Vielfalt wird meine späteren Berufschan- } \\
\text { cen verringern. }\end{array}$ & $\square$ & $\square$ & $\square$ & $\square$ \\
\hline
\end{tabular}




\begin{tabular}{|c|c|c|c|c|}
\hline & $\begin{array}{c}\text { Stimme } \\
\mathrm{zu}\end{array}$ & $\begin{array}{c}\text { Stimme } \\
\text { weitgehend } \\
\mathrm{zu}\end{array}$ & $\begin{array}{l}\text { Stimme } \\
\text { teilweise } \\
\text { zu }\end{array}$ & $\begin{array}{l}\text { Stimme } \\
\text { nicht } \\
\text { zu }\end{array}$ \\
\hline $\begin{array}{l}\text { 12. Innerhalb der nächsten Jahrzehnte } \\
\text { werden Tausende Arten aussterben, was } \\
\text { vor allem schlimme Konsequenzen für die } \\
\text { Natur hat. }\end{array}$ & $\square$ & $\square$ & $\square$ & $\square$ \\
\hline $\begin{array}{l}\text { 13. Das Artensterben, dessen Ursachen } \\
\text { hier bei uns liegen, wird allen Menschen auf } \\
\text { der Erde schaden. }\end{array}$ & $\square$ & $\square$ & $\square$ & $\square$ \\
\hline $\begin{array}{l}\text { 14. Wenn eine Art ausstirbt, wird dies vor } \\
\text { allem andere Arten beeinträchtigen und so } \\
\text { den Verlust der biologischen Vielfalt wei- } \\
\text { ter verschlimmern. }\end{array}$ & $\square$ & $\square$ & $\square$ & $\square$ \\
\hline $\begin{array}{l}\text { 15. Verstärkter Schutz der biologischen } \\
\text { Vielfalt wird den Menschen in Entwick- } \\
\text { lungsländern schaden, die direkt von Pflan- } \\
\text { zen und Tieren abhängen. }\end{array}$ & $\square$ & $\square$ & $\square$ & $\square$ \\
\hline
\end{tabular}




\section{Die Teufelskralle - aktueller Biodiversitätskonflikt}
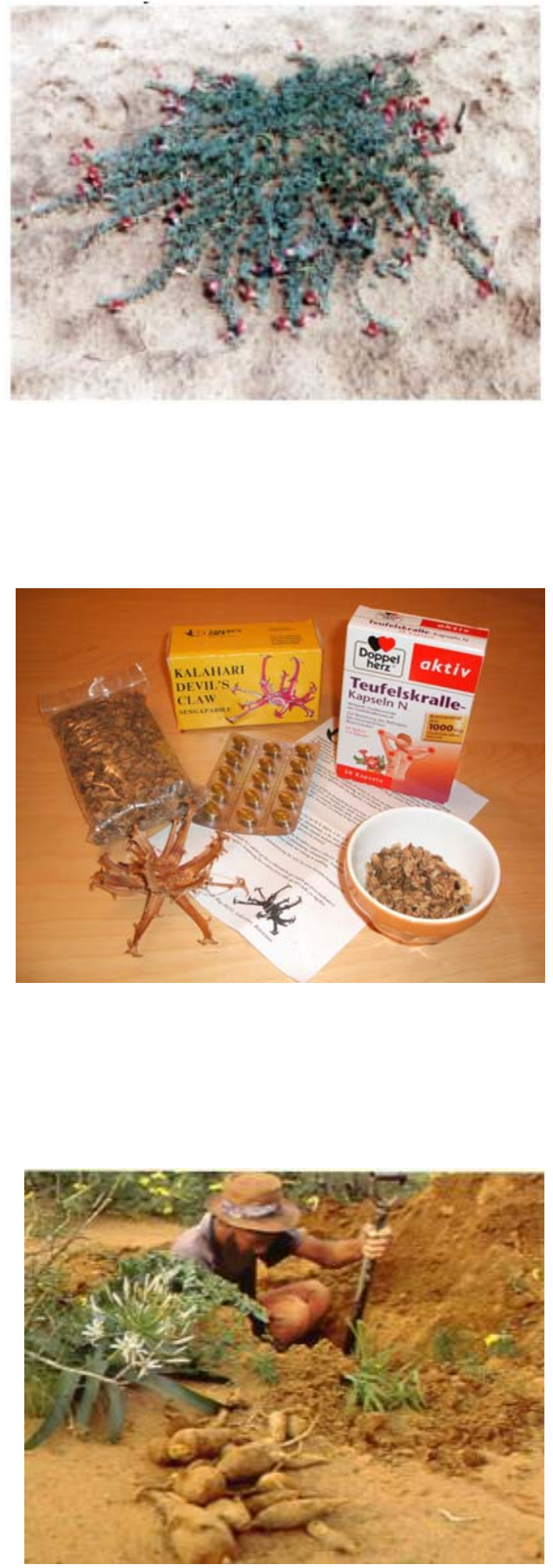

Die afrikanische Teufelskralle (Harpagophytum procumbens) wächst in der Kalahari, einer Wüste im Süden Afrikas. Die Pflanze wächst dort wild und kommt in der Natur in keiner anderen Region der Erde vor. Sie ist an die klimatischen Verhältnisse der Kalahari recht gut angepasst, vor allem an Trockenheit.

Die Pflanze ist ein beliebtes und effizientes Heilmittel gegen Rheuma. Traditionell wird sie von den Bewohner(inne)n des südlichen Afrikas seit Tausenden Jahren erfolgreich als Heilmittel eingesetzt.

Die Teufelskralle wird heutzutage vor allem auf europäischen und nordamerikanischen Märkten verkauft. Man kann Teufelskralle Produkte in Form von Tees, Tabletten oder als Pulver erwerben.

Die Pflanze wird von Sammler(inne)n der lokalen Bevölkerung geerntet. Die Teufelskralle speichert Wasser in ihren verdickten Wurzeln und kann so Trockenphasen überdauern. Inhaltsstoffe aus den Wurzeln der Pflanze werden verwendet und zu Medikamenten verarbeitet. Die Sammler(innen) verkaufen die Pflanzenwurzeln meistens an Zwischenhändler, die dann die Wurzeln an europäische Arzneimittelhersteller verkaufen.

Viele Menschen im südlichen Afrika verschaffen sich durch den Verkauf von wild gesammelten Wurzeln der Teufelskralle ein Einkommen. Die Preise, die für die gesammelten Wurzeln gezahlt werden, sind gering. Für die ärmere Bevölkerung ist das Sammeln und Verkaufen der Teufelskralle jedoch die einzige Einkommensmöglichkeit.

Die Nachfrage nach Teufelskralle Produkten ist stark angestiegen. Der Export von Teufelskrallen Wurzeln aus Afrika hat daher stark zugenommen Die Bestände der Pflanze in der Kalahari gehen mittlerweile vermutlich stark zurück. 


\section{Achtung!}

Bei den folgenden Fragen gibt es zur Beantwortung einer Aussage zwei Blöcke: Einen grauen Block, der sich auf die gesamte Welt bezieht, einen weißen Block, der sich auf die Region bezieht, in der Sie leben. Hier bitte in jedem Block ein Kreuz machen, also zwei Kreuze pro Zeile!

\section{Beispiel:}

\begin{tabular}{|c|c|c|c|c|c|c|c|c|}
\hline & \multicolumn{4}{|c|}{ Im grauen Block ein Kreuz pro Zeile $\downarrow$} & \multicolumn{4}{|c|}{$\begin{array}{c}\ldots \text { und im weißen Block ein Kreuz pro } \\
\text { Zeile } \downarrow\end{array}$} \\
\hline & $\begin{array}{c}\text { Stimme } \\
\mathrm{zu}\end{array}$ & $\begin{array}{l}\text { Stimme } \\
\text { weitgehend } \\
\text { zu }\end{array}$ & $\begin{array}{l}\text { Stimme } \\
\text { teilweise } \\
\text { zu }\end{array}$ & $\begin{array}{l}\text { Stimme } \\
\text { nicht } \\
\text { zu }\end{array}$ & $\begin{array}{c}\text { Stimme } \\
\text { zu }\end{array}$ & $\begin{array}{l}\text { Stimme } \\
\text { weitgehend } \\
\text { zu }\end{array}$ & $\begin{array}{l}\text { Stimme } \\
\text { teilweise } \\
\text { zu }\end{array}$ & $\begin{array}{c}\text { Stimme } \\
\text { nicht } \\
\text { zu }\end{array}$ \\
\hline & \multicolumn{4}{|c|}{ auf der Welt } & \multicolumn{4}{|c|}{ in unserer Region } \\
\hline $\begin{array}{l}\text { Ich kenne viele Tierarten auf der Welt/in unse- } \\
\text { rer Region. }\end{array}$ & $\square$ & 凶 & $\square$ & $\square$ & 凶 & $\square$ & $\square$ & $\square$ \\
\hline
\end{tabular}


II. Im Folgenden sehen Sie einige Aussagen zu dem Problem des Rückgangs der biologischen Vielfalt. Sie können jeweils ankreuzen, inwiefern Sie den Gründen für den Verlust der biologischen Vielfalt auf der Welt (grauer Block) und in der Region, in der Sie leben (weißer Block) zustimmen.

\begin{tabular}{|c|c|c|c|c|c|c|c|c|}
\hline \multirow[b]{2}{*}{$\begin{array}{l}\text { Biologische Vielfalt ist hauptsächlich } \\
\text { gefährdet }\end{array}$} & \multicolumn{4}{|c|}{$\begin{array}{c}\text { Im grauen Block ein Kreuz pro } \\
\text { Zeile } \downarrow\end{array}$} & \multicolumn{4}{|c|}{$\begin{array}{c}\ldots . \text { und im weißen Block ein Kreuz pro } \\
\text { Zeile } \downarrow\end{array}$} \\
\hline & $\begin{array}{l}\text { Stimme } \\
\mathrm{zu}\end{array}$ & $\begin{array}{c}\text { Stimme } \\
\text { weitgehend } \\
\text { zu }\end{array}$ & $\begin{array}{l}\text { Stimme } \\
\text { teilweise } \\
\text { zu }\end{array}$ & $\begin{array}{l}\text { Stimme } \\
\text { nicht } \\
\text { zu }\end{array}$ & $\begin{array}{l}\text { Stimme } \\
\mathrm{zu}\end{array}$ & $\begin{array}{l}\text { Stimme } \\
\text { weitgehend } \\
\text { zu }\end{array}$ & $\begin{array}{l}\text { Stimme } \\
\text { teilweise } \\
\text { zu }\end{array}$ & $\begin{array}{l}\text { Stimme } \\
\text { nicht } \\
\mathrm{zu}\end{array}$ \\
\hline & \multicolumn{4}{|c|}{ auf der Welt } & \multicolumn{4}{|c|}{ in unserer Region } \\
\hline $\begin{array}{l}\text { 2. ...da das Aussterben einer Art auf der } \\
\text { Welt/in unserer Region auch weitere Arten } \\
\text { gefährden kann. }\end{array}$ & $\square$ & $\square$ & $\square$ & $\square$ & $\square$ & $\square$ & $\square$ & $\square$ \\
\hline $\begin{array}{l}\text { 3. ...durch die Armut von Menschen auf der } \\
\text { Welt/in unserer Region, die Pflanzen und Tie- } \\
\text { re verkaufen müssen. }\end{array}$ & $\square$ & $\square$ & $\square$ & $\square$ & $\square$ & $\square$ & $\square$ & $\square$ \\
\hline $\begin{array}{l}\text { 5. ...weil wir Menschen nicht genug über die } \\
\text { Gefährdung der biologischen Vielfalt auf der } \\
\text { Welt/in unserer Region wissen. }\end{array}$ & $\square$ & $\square$ & $\square$ & $\square$ & $\square$ & $\square$ & $\square$ & $\square$ \\
\hline
\end{tabular}




\begin{tabular}{|c|c|c|c|c|c|c|c|c|}
\hline \multirow{3}{*}{$\begin{array}{l}\text { Biologische Vielfalt ist hauptsächlich } \\
\text { gefährdet }\end{array}$} & \multicolumn{4}{|c|}{$\begin{array}{c}\text { Im grauen Block ein Kreuz pro } \\
\text { Zeile } \downarrow\end{array}$} & \multicolumn{4}{|c|}{$\begin{array}{c}\ldots . \text { und im weißen Block ein Kreuz pro } \\
\text { Zeile } \downarrow\end{array}$} \\
\hline & $\underset{\mathrm{zu}}{\text { Stimme }}$ & $\begin{array}{l}\text { Stimme } \\
\text { weitgehend } \\
\text { zu }\end{array}$ & $\begin{array}{l}\text { Stimme } \\
\text { teilweise } \\
\text { zu }\end{array}$ & $\begin{array}{l}\text { Stimme } \\
\text { nicht } \\
\text { zu }\end{array}$ & $\underset{\mathrm{zu}}{\text { Stimme }}$ & $\begin{array}{l}\text { Stimme } \\
\text { weitgehend } \\
\text { zu }\end{array}$ & $\begin{array}{l}\text { Stimme } \\
\text { teilweise } \\
\text { zu }\end{array}$ & $\begin{array}{c}\text { Stimme } \\
\text { nicht } \\
\text { zu }\end{array}$ \\
\hline & \multicolumn{4}{|c|}{ auf der Welt } & \multicolumn{4}{|c|}{ in unserer Region } \\
\hline $\begin{array}{l}\text { 7. ... weil viele Produkte aus Tieren oder Pflan- } \\
\text { zen auf der Welt/in unserer Region so billig } \\
\text { sind, dass sie sich jede(r) leisten kann. }\end{array}$ & $\square$ & $\square$ & $\square$ & $\square$ & $\square$ & $\square$ & $\square$ & $\square$ \\
\hline $\begin{array}{l}\text { 8. ... weil es nicht genügend Lebensräume auf } \\
\text { der Welt/in unserer Region gibt, in denen } \\
\text { sich Tiere und Pflanzen ungestört vermehren } \\
\text { können. }\end{array}$ & $\square$ & $\square$ & $\square$ & $\square$ & $\square$ & $\square$ & $\square$ & $\square$ \\
\hline $\begin{array}{l}\text { 9. ... weil Gesetze zum Schutz der biologischen } \\
\text { Vielfalt vor übermäßigem Handel auf der } \\
\text { Welt/in unserer Region nicht ausreichen. }\end{array}$ & $\square$ & $\square$ & $\square$ & $\square$ & $\square$ & $\square$ & $\square$ & $\square$ \\
\hline $\begin{array}{l}\text { 10. ... da wir Menschen nicht bereit sind, hö- } \\
\text { here Preise für Produkte zu zahlen, die die } \\
\text { biologische Vielfalt auf der Welt/in unserer } \\
\text { Region schonen. }\end{array}$ & $\square$ & $\square$ & $\square$ & $\square$ & $\square$ & $\square$ & $\square$ & $\square$ \\
\hline
\end{tabular}




\begin{tabular}{|c|c|c|c|c|c|c|c|c|}
\hline \multirow[b]{2}{*}{$\begin{array}{l}\text { Biologische Vielfalt ist hauptsächlich } \\
\text { gefährdet }\end{array}$} & \multicolumn{4}{|c|}{$\begin{array}{c}\text { Im grauen Block ein Kreuz pro } \\
\text { Zeile } \downarrow\end{array}$} & \multicolumn{4}{|c|}{$\begin{array}{c}\ldots . \text { und im weißen Block ein Kreuz pro } \\
\text { Zeile } \downarrow\end{array}$} \\
\hline & $\begin{array}{c}\text { Stimme } \\
\mathrm{zu}\end{array}$ & $\begin{array}{l}\text { Stimme } \\
\text { weitgehend } \\
\text { zu }\end{array}$ & $\begin{array}{l}\text { Stimme } \\
\text { teilweise } \\
\text { zu }\end{array}$ & $\begin{array}{l}\text { Stimme } \\
\text { nicht } \\
\text { zu }\end{array}$ & $\underset{\mathrm{zu}}{\text { Stimme }}$ & $\begin{array}{l}\text { Stimme } \\
\text { weitgehend } \\
\text { zu }\end{array}$ & $\begin{array}{l}\text { Stimme } \\
\text { teilweise } \\
\mathrm{zu}\end{array}$ & $\begin{array}{l}\text { Stimme } \\
\text { nicht } \\
\text { zu }\end{array}$ \\
\hline & \multicolumn{4}{|c|}{ auf der Welt } & \multicolumn{4}{|c|}{ in unserer Region } \\
\hline $\begin{array}{l}\text { 12. ...durch Menschen auf der Welt/in unse- } \\
\text { rer Region, die unbewusst Produkte mit Zu- } \\
\text { sätzen bedrohter Pflanzen und Tiere kaufen. }\end{array}$ & $\square$ & $\square$ & $\square$ & $\square$ & $\square$ & $\square$ & $\square$ & $\square$ \\
\hline
\end{tabular}


Bitte kreuzen Sie auch hier an, inwieweit Sie diesen Aussagen zum Verlust der biologischen Vielfalt auf der Welt und in der Region, in der Sie leben, zustimmen.

\begin{tabular}{|c|c|c|c|c|c|c|c|c|}
\hline & \multicolumn{4}{|c|}{$\begin{array}{c}\text { Im grauen Block ein Kreuz pro } \\
\text { Zeile } \downarrow\end{array}$} & \multicolumn{4}{|c|}{$\begin{array}{c}\ldots . \text { und im weißen Block ein Kreuz pro } \\
\text { Zeile } \downarrow\end{array}$} \\
\hline & $\underset{\mathrm{zu}}{\text { Stimme }}$ & $\begin{array}{l}\text { Stimme } \\
\text { weitgehend } \\
\mathrm{zu}\end{array}$ & $\begin{array}{l}\text { Stimme } \\
\text { teilweise } \\
\text { zu }\end{array}$ & $\begin{array}{l}\text { Stimme } \\
\text { nicht } \\
\text { zu }\end{array}$ & $\begin{array}{c}\text { Stimme } \\
\mathrm{zu}\end{array}$ & $\begin{array}{l}\text { Stimme } \\
\text { weitgehend } \\
\mathrm{zu}\end{array}$ & $\begin{array}{l}\text { Stimme } \\
\text { teilweise } \\
\text { zu }\end{array}$ & $\begin{array}{l}\text { Stimme } \\
\text { nicht } \\
\text { zu }\end{array}$ \\
\hline & \multicolumn{4}{|c|}{ auf der Welt } & \multicolumn{4}{|c|}{ in unserer Region } \\
\hline $\begin{array}{l}\text { 14. Ich glaube, dass nicht so viele Arten auf der } \\
\text { Welt/in unserer Region direkt vom Aussterben } \\
\text { bedroht sind. }\end{array}$ & $\square$ & $\square$ & $\square$ & $\square$ & $\square$ & $\square$ & $\square$ & $\square$ \\
\hline $\begin{array}{l}\text { 15. Vieles wird übertrieben dargestellt und be- } \\
\text { droht die biologische Vielfalt auf der Welt/in } \\
\text { unserer Region nicht wirklich. }\end{array}$ & $\square$ & $\square$ & $\square$ & $\square$ & $\square$ & $\square$ & $\square$ & $\square$ \\
\hline
\end{tabular}


III. Was kann man tun, um die biologische Vielfalt zu bewahren?

Mit dieser Frage befassen sich die nun folgenden Aussagen. Bitte beachten Sie: Wenn von „Kosmetika” die Rede ist, sind auch alltägliche Körperpflegemittel, wie Deodorants, Seife, Rasierschaum oder Shampoo gemeint. Bitte drücken Sie auch hier wieder Ihre Zustimmung oder nicht Zustimmung jeweils für die Welt und für Ihre Region aus.

\begin{tabular}{|c|c|c|c|c|c|c|c|c|}
\hline & \multicolumn{4}{|c|}{$\begin{array}{c}\text { Im grauen Block ein Kreuz pro } \\
\text { Zeile } \downarrow\end{array}$} & \multicolumn{4}{|c|}{$\begin{array}{c}\ldots \text { und im weißen Block ein Kreuz pro } \\
\text { Zeile } \downarrow\end{array}$} \\
\hline & $\begin{array}{c}\text { Stimme } \\
\text { zu }\end{array}$ & $\begin{array}{c}\text { Stimme } \\
\text { weitgehend } \\
\mathrm{zu}\end{array}$ & $\begin{array}{c}\text { Stimme } \\
\text { teilweise } \\
\text { zu }\end{array}$ & $\begin{array}{c}\text { Stimme } \\
\text { nicht } \\
\text { zu }\end{array}$ & $\begin{array}{c}\text { Stimme } \\
\mathrm{zu}\end{array}$ & $\begin{array}{c}\text { Stimme } \\
\text { weitgehend } \\
\text { zu }\end{array}$ & $\begin{array}{c}\text { Stimme } \\
\text { teilweise } \\
\text { zu }\end{array}$ & $\begin{array}{c}\text { Stimme } \\
\text { nicht } \\
\mathrm{zu}\end{array}$ \\
\hline & \multicolumn{4}{|c|}{ auf der Welt } & \multicolumn{4}{|c|}{ in unserer Region } \\
\hline $\begin{array}{l}\text { 2. Käufer wie ich sind dafür verantwortlich, } \\
\text { dass die biologische Vielfalt auf der Welt/in } \\
\text { unserer Region durch Verarbeitung in Kosme- } \\
\text { tika gefährdet ist. }\end{array}$ & $\square$ & $\square$ & $\square$ & $\square$ & $\square$ & $\square$ & $\square$ & $\square$ \\
\hline $\begin{array}{l}\text { 4. Ich fühle mich verantwortlich, Arten auf } \\
\text { der Welt/in unserer Region zu schützen. }\end{array}$ & $\square$ & $\square$ & $\square$ & $\square$ & $\square$ & $\square$ & $\square$ & $\square$ \\
\hline
\end{tabular}




\begin{tabular}{|c|c|c|c|c|c|c|c|c|}
\hline & \multicolumn{4}{|c|}{$\begin{array}{c}\text { Im grauen Block ein Kreuz pro } \\
\text { Zeile } \downarrow\end{array}$} & \multicolumn{4}{|c|}{$\begin{array}{c}\ldots . \text { und im weißen Block ein Kreuz pro } \\
\text { Zeile } \downarrow\end{array}$} \\
\hline & $\begin{array}{c}\text { Stimme } \\
\mathrm{zu}\end{array}$ & $\begin{array}{l}\text { Stimme } \\
\text { weitgehend } \\
\text { zu }\end{array}$ & $\begin{array}{l}\text { Stimme } \\
\text { teilweise } \\
\text { zu }\end{array}$ & $\begin{array}{l}\text { Stimme } \\
\text { nicht } \\
\text { zu }\end{array}$ & $\underset{\mathrm{zu}}{\text { Stimme }}$ & $\begin{array}{l}\text { Stimme } \\
\text { weitgehend } \\
\text { zu }\end{array}$ & $\begin{array}{l}\text { Stimme } \\
\text { teilweise } \\
\text { zu }\end{array}$ & $\begin{array}{l}\text { Stimme } \\
\text { nicht } \\
\text { zu }\end{array}$ \\
\hline & \multicolumn{4}{|c|}{ auf der Welt } & \multicolumn{4}{|c|}{ in unserer Region } \\
\hline $\begin{array}{l}\text { 5. Jede/r einzelne kann durch sorgfältige } \\
\text { Auswahl von Heilmitteln, die man kauft zum } \\
\text { Schutz von gefährdeten Medizinalpflanzen } \\
\text { auf der Welt/in unserer Region beitragen. }\end{array}$ & $\square$ & $\square$ & $\square$ & $\square$ & $\square$ & $\square$ & $\square$ & $\square$ \\
\hline $\begin{array}{l}\text { 6. Durch gezielte Auswahl von Kosmetika } \\
\text { kann ich dazu beitragen, die gefährdete bio- } \\
\text { logische Vielfalt auf der Welt/in unserer } \\
\text { Region zu erhalten. }\end{array}$ & $\square$ & $\square$ & $\square$ & $\square$ & $\square$ & $\square$ & $\square$ & $\square$ \\
\hline $\begin{array}{l}\text { 8. Jeder Mensch, auch ich kann dazu beitra- } \\
\text { gen, die Arten auf der Welt/in unserer Regi- } \\
\text { on zu schützen. }\end{array}$ & $\square$ & $\square$ & $\square$ & $\square$ & $\square$ & $\square$ & $\square$ & $\square$ \\
\hline
\end{tabular}


IV. Hier sehen Sie Aussagen über die Verbindung von Mensch und Umwelt. Für jede dieser Aussagen kreuzen Sie bitte an, inwieweit Sie zustimmen.

\begin{tabular}{|c|c|c|c|c|}
\hline & $\begin{array}{c}\text { Stimme } \\
\mathrm{zu}\end{array}$ & $\begin{array}{l}\text { Stimme } \\
\text { weitge- } \\
\text { hend zu }\end{array}$ & $\begin{array}{c}\text { Stimme } \\
\text { teilweise } \\
\text { zu }\end{array}$ & $\begin{array}{c}\text { Stimme } \\
\text { nicht } \\
\text { zu }\end{array}$ \\
\hline $\begin{array}{l}\text { 1. Wir nähern uns der Grenze der Bevölkerungs- } \\
\text { zahl, die die Erde tragen kann. }\end{array}$ & $\square$ & $\square$ & $\square$ & $\square$ \\
\hline $\begin{array}{l}\text { 2. Menschen haben das Recht, die Natur für ihre } \\
\text { Bedürfnisse zu verändern. }\end{array}$ & $\square$ & $\square$ & $\square$ & $\square$ \\
\hline $\begin{array}{l}\text { 3. Wenn Menschen in die Natur eingreifen, hat } \\
\text { es meistens verheerende Folgen. }\end{array}$ & $\square$ & $\square$ & $\square$ & $\square$ \\
\hline $\begin{array}{l}\text { 4. Der Einfallsreichtum der Menschen wird dazu } \\
\text { führen, dass die Erde NICHT unbewohnbar wird. }\end{array}$ & $\square$ & $\square$ & $\square$ & $\square$ \\
\hline 5. Menschen missbrauchen die Umwelt stark. & $\square$ & $\square$ & $\square$ & $\square$ \\
\hline $\begin{array}{l}\text { 6. Die Erde hat eine Fülle natürlicher Ressour- } \\
\text { cen, wir müssen nur lernen, sie zu nutzen. }\end{array}$ & $\square$ & $\square$ & $\square$ & $\square$ \\
\hline $\begin{array}{l}\text { 7. Pflanzen und Tiere haben das gleiche Recht zu } \\
\text { existieren wie Menschen. }\end{array}$ & $\square$ & $\square$ & $\square$ & $\square$ \\
\hline $\begin{array}{l}\text { 8. Die Balance der Natur ist stark genug, um mit } \\
\text { den Einflüssen von modernen Industrieländern } \\
\text { zurecht zu kommen. }\end{array}$ & $\square$ & $\square$ & $\square$ & $\square$ \\
\hline $\begin{array}{l}\text { 9. Trotz unserer besonderen Fähigkeiten unter- } \\
\text { liegen wir Menschen immer noch den Naturge- } \\
\text { setzen. }\end{array}$ & $\square$ & $\square$ & $\square$ & $\square$ \\
\hline $\begin{array}{l}\text { 10. Die sogenannte "ökologische Krise", der wir } \\
\text { Menschen gegenübergestellt sind, wird stark } \\
\text { übertrieben. }\end{array}$ & $\square$ & $\square$ & $\square$ & $\square$ \\
\hline $\begin{array}{l}\text { 11. Die Erde ist wie ein Raumschiff mit sehr be- } \\
\text { grenztem Raum und Ressourcen. }\end{array}$ & $\square$ & $\square$ & $\square$ & $\square$ \\
\hline $\begin{array}{l}\text { 12. Menschen sind dazu bestimmt, über den Rest } \\
\text { der Natur zu herrschen. }\end{array}$ & $\square$ & $\square$ & $\square$ & $\square$ \\
\hline $\begin{array}{l}\text { 13. Die Balance der Natur ist sehr empfindlich } \\
\text { und leicht zu stören. }\end{array}$ & $\square$ & $\square$ & $\square$ & $\square$ \\
\hline $\begin{array}{l}\text { 14. Die Menschen werden letztendlich genug } \\
\text { darüber wissen, wie die Natur funktioniert und } \\
\text { so in der Lage sein, sie zu kontrollieren. }\end{array}$ & $\square$ & $\square$ & $\square$ & $\square$ \\
\hline $\begin{array}{l}\text { 15. Wenn alles so weiter geht wie im Moment, } \\
\text { werden wir bald eine gewaltige ökologische Ka- } \\
\text { tastrophe erleben. }\end{array}$ & $\square$ & $\square$ & $\square$ & $\square$ \\
\hline
\end{tabular}


V. Wir möchten Sie bitten, folgende Fragen wieder zum einen für die gesamte Welt (im grauen Feld), zum anderen für die Region, in der Sie leben (im weißen Feld) zu beantworten.

\begin{tabular}{|c|c|c|c|c|c|c|c|c|}
\hline & \multicolumn{4}{|c|}{$\begin{array}{c}\text { Im grauen Block ein Kreuz pro } \\
\text { Zeile } \downarrow\end{array}$} & \multicolumn{4}{|c|}{$\begin{array}{c}\ldots \text { und im weißen Block ein Kreuz pro } \\
\text { Zeile } \downarrow\end{array}$} \\
\hline & $\underset{\mathrm{zu}}{\text { Stimme }}$ & $\begin{array}{c}\text { Stimme } \\
\text { weitgehend } \\
\text { zu }\end{array}$ & $\begin{array}{l}\text { Stimme } \\
\text { teilweise } \\
\text { zu }\end{array}$ & $\begin{array}{l}\text { Stimme } \\
\text { nicht } \\
\text { zu }\end{array}$ & $\underset{\mathrm{zu}}{\text { Stimme }}$ & $\begin{array}{c}\text { Stimme } \\
\text { weitgehend } \\
\text { zu }\end{array}$ & $\begin{array}{l}\text { Stimme } \\
\text { teilweise } \\
\text { zu }\end{array}$ & $\begin{array}{c}\text { Stimme } \\
\text { nicht } \\
\text { zu }\end{array}$ \\
\hline & \multicolumn{4}{|c|}{ auf der Welt } & \multicolumn{4}{|c|}{ in unserer Region } \\
\hline $\begin{array}{l}\text { 2. Ich fühle mich schuldig, wenn ich wissent- } \\
\text { lich Medikamente aus gefährdeten Medizi- } \\
\text { nalpflanzen auf der Welt/in unserer Region } \\
\text { kaufe. }\end{array}$ & $\square$ & $\square$ & $\square$ & $\square$ & $\square$ & $\square$ & $\square$ & $\square$ \\
\hline $\begin{array}{l}\text { 4. Mein Gewissen sagt mir, dass ich mehr tun } \\
\text { sollte, um Arten auf der Welt/in unserer Re- } \\
\text { gion zu schützen. }\end{array}$ & $\square$ & $\square$ & $\square$ & $\square$ & $\square$ & $\square$ & $\square$ & $\square$ \\
\hline
\end{tabular}


VI. Es kommen nun einige Aussagen dazu, was man tun kann, um die biologische Vielfalt zu schützen. Wir interessieren uns dafür, was Sie tun würden.

\begin{tabular}{|c|c|c|c|c|}
\hline Ich würde & $\underset{\mathrm{zu}}{\text { Stimme }}$ & $\begin{array}{l}\text { Stimme } \\
\text { weitgehend } \\
\text { zu }\end{array}$ & $\begin{array}{l}\text { Stimme } \\
\text { teilweise } \\
\text { zu }\end{array}$ & $\begin{array}{c}\text { Stimme } \\
\text { nicht } \\
\text { zu }\end{array}$ \\
\hline $\begin{array}{l}\text { 1. ...einen Brief an die Regierung schreiben, } \\
\text { um auf den notwendigen Schutz der biologi- } \\
\text { schen Vielfalt hinzuweisen. }\end{array}$ & $\square$ & $\square$ & $\square$ & $\square$ \\
\hline $\begin{array}{l}\text { 2. ...selbst Arten schützen (z.B. beim Wan- } \\
\text { dern die Wege nicht verlassen, um die Arten } \\
\text { dort nicht zu stören.) }\end{array}$ & $\square$ & $\square$ & $\square$ & $\square$ \\
\hline $\begin{array}{l}\text { 3. ...die Marke der Kosmetika wechseln, wenn } \\
\text { ich erfahre, dass die Herstellung die biologi- } \\
\text { sche Vielfalt gefährdet. }\end{array}$ & $\square$ & $\square$ & $\square$ & $\square$ \\
\hline $\begin{array}{l}\text { 4. ... Protest-Transparente vor der Zentrale } \\
\text { einer Firma anbringen, von der ich weiß, dass } \\
\text { sie zum Artensterben beiträgt. }\end{array}$ & $\square$ & $\square$ & $\square$ & $\square$ \\
\hline $\begin{array}{l}\text { 5. ...bei einer Umweltgruppe in unserer Schule } \\
\text { mitmachen, die Artenschutzprojekte durch- } \\
\text { führen würde. }\end{array}$ & $\square$ & $\square$ & $\square$ & $\square$ \\
\hline $\begin{array}{l}\text { 6. ... für Kosmetika mehr bezahlen, wenn damit } \\
\text { gesichert wäre, dass sie die biologische Viel- } \\
\text { falt nicht gefährden. }\end{array}$ & $\square$ & $\square$ & $\square$ & $\square$ \\
\hline $\begin{array}{l}\text { 7. ...offensiv Flugblätter verteilen, die über } \\
\text { den Rückgang biologischer Vielfalt durch de- } \\
\text { ren Verwendung in Kosmetika aufklären. }\end{array}$ & $\square$ & $\square$ & $\square$ & $\square$ \\
\hline $\begin{array}{l}\text { 8. ...eine Unterschriftenliste zum Schutz von } \\
\text { gefährdeten Arten unterzeichnen. }\end{array}$ & $\square$ & $\square$ & $\square$ & $\square$ \\
\hline $\begin{array}{l}\text { 9. ...gegen die Verwendung von gefährdeten } \\
\text { Medizinalpflanzen in Medikamenten öffent- } \\
\text { lich demonstrieren. }\end{array}$ & $\square$ & $\square$ & $\square$ & $\square$ \\
\hline $\begin{array}{l}\text { 10....keine Medikamente kaufen, die aus ge- } \\
\text { fährdeten Medizinalpflanzen hergestellt } \\
\text { werden. }\end{array}$ & $\square$ & $\square$ & $\square$ & $\square$ \\
\hline $\begin{array}{l}\text { 11. ...bei einer Gruppe mitmachen, die unserer } \\
\text { Schule ein neues Profil mit dem Schwerpunkt } \\
\text { "Schutz der biologischen Vielfalt" geben } \\
\text { möchte. }\end{array}$ & $\square$ & $\square$ & $\square$ & $\square$ \\
\hline
\end{tabular}




\begin{tabular}{|c|c|c|c|c|}
\hline Ich würde & $\begin{array}{c}\text { Stimme } \\
\mathrm{zu}\end{array}$ & $\begin{array}{l}\text { Stimme } \\
\text { weitgehend } \\
\text { zu }\end{array}$ & $\begin{array}{l}\text { Stimme } \\
\text { teilweise } \\
\text { zu }\end{array}$ & $\begin{array}{l}\text { Stimme } \\
\text { nicht } \\
\text { zu }\end{array}$ \\
\hline $\begin{array}{l}\text { 12. ... direkt selbst zum Schutz der biologi- } \\
\text { schen Vielfalt beitragen (zum Beispiel durch } \\
\text { die Verwilderung eines Stück Gartens.) }\end{array}$ & $\square$ & $\square$ & $\square$ & $\square$ \\
\hline $\begin{array}{l}\text { 13. ...es begrüßen, wenn der Staat gefährdete } \\
\text { Medizinalpflanzen vor Verwendung in Medi- } \\
\text { kamenten gesetzlich schützt. }\end{array}$ & $\square$ & $\square$ & $\square$ & $\square$ \\
\hline $\begin{array}{l}\text { 14. ... an einem Informationsstand Passanten } \\
\text { aktiv ansprechen und über die Bedrohung der } \\
\text { biologischen Vielfalt engagiert diskutieren. }\end{array}$ & $\square$ & $\square$ & $\square$ & $\square$ \\
\hline
\end{tabular}


Nun sind wir fast am Ende des Fragebogens angelangt. Es folgen nun noch einige ganz allgemeine Fragen zu Dingen, die Ihnen wichtig sind im Leben.

Im Folgenden finden Sie kurze Beschreibungen einiger Menschen. Bitte lesen Sie jede Beschreibung und überlegen Sie dann, wie sehr jede Person wie Sie bzw. nicht wie Sie ist.

\begin{tabular}{|c|c|c|c|c|c|c|}
\hline & $\begin{array}{l}\text { sehr } \\
\text { wie } \\
\text { ich }\end{array}$ & $\begin{array}{l}\text { wie } \\
\text { ich }\end{array}$ & $\begin{array}{l}\text { etwa } \\
\text { wie } \\
\text { ich }\end{array}$ & $\begin{array}{l}\text { ein } \\
\text { wenig } \\
\text { wie } \\
\text { ich }\end{array}$ & $\begin{array}{l}\text { nicht } \\
\text { wie } \\
\text { ich }\end{array}$ & $\begin{array}{l}\text { über- } \\
\text { haupt } \\
\text { nicht } \\
\text { wie } \\
\text { ich }\end{array}$ \\
\hline $\begin{array}{l}\text { 1. Sich neue Ideen auszudenken und } \\
\text { kreativ zu sein ist wichtig für sie/ihn. } \\
\text { Sie/Er mag es, Dinge auf seine/ihre } \\
\text { eigene originelle Art und Weise zu tun. }\end{array}$ & $\square$ & $\square$ & $\square$ & $\square$ & $\square$ & $\square$ \\
\hline $\begin{array}{l}\text { 2. Es ist wichtig für sielihn, reich zu } \\
\text { sein. SielEr möchte eine Menge Geld } \\
\text { und teure Dinge haben. }\end{array}$ & $\square$ & $\square$ & $\square$ & $\square$ & $\square$ & $\square$ \\
\hline $\begin{array}{l}\text { 3. Sie/Er denkt, dass es wichtig ist je- } \\
\text { den Mensch auf der Welt gleich zu be- } \\
\text { handeln. Sieler meint, dass jedelr im } \\
\text { Leben die gleichen Möglichkeiten haben } \\
\text { sollte. }\end{array}$ & $\square$ & $\square$ & $\square$ & $\square$ & $\square$ & $\square$ \\
\hline $\begin{array}{l}\text { 4. Für sielihn ist es sehr wichtig, } \\
\text { ihre/seine Fähigkeiten zu zeigen. Sie/Er } \\
\text { möchte, dass Menschen bewundern, was } \\
\text { er/sie tut. }\end{array}$ & $\square$ & $\square$ & $\square$ & $\square$ & $\square$ & $\square$ \\
\hline $\begin{array}{l}\text { 5. Es ist wichtig für sielihn, in einem } \\
\text { sicheren Umfeld zu leben. Sie/Er } \\
\text { vermeidet alles, was ihre/seine } \\
\text { Sicherheit gefährden könnte. }\end{array}$ & $\square$ & $\square$ & $\square$ & $\square$ & $\square$ & $\square$ \\
\hline $\begin{array}{l}\text { 6. Sie/Er denkt, dass es wichtig ist, im } \\
\text { Leben viele verschiedene Dinge zu tun. } \\
\text { SielEr sucht immer nach neuen Dingen, } \\
\text { die sieler ausprobieren kann. }\end{array}$ & $\square$ & $\square$ & $\square$ & $\square$ & $\square$ & $\square$ \\
\hline $\begin{array}{l}\text { 7. Sie/Er meint, dass Menschen tun } \\
\text { sollen, was ihnen gesagt wird. Sie/Er } \\
\text { denkt, dass Menschen sich immer an die } \\
\text { Regeln halten sollten, auch wenn sie } \\
\text { niemand beobachtet. }\end{array}$ & $\square$ & $\square$ & $\square$ & $\square$ & $\square$ & $\square$ \\
\hline $\begin{array}{l}\text { 8. Für sielihn ist es wichtig, Menschen } \\
\text { zuzuhören, die anders sind als sieler. } \\
\text { Auch wenn sieler nicht mit ihnen über- } \\
\text { einstimmt, möchte sieler sie dennoch } \\
\text { verstehen. }\end{array}$ & $\square$ & $\square$ & $\square$ & $\square$ & $\square$ & $\square$ \\
\hline
\end{tabular}




\begin{tabular}{|c|c|c|c|c|c|c|}
\hline & $\begin{array}{l}\text { sehr } \\
\text { wie } \\
\text { ich }\end{array}$ & $\begin{array}{l}\text { wie } \\
\text { ich }\end{array}$ & $\begin{array}{l}\text { etwa } \\
\text { wie } \\
\text { ich }\end{array}$ & $\begin{array}{l}\text { ein } \\
\text { wenig } \\
\text { wie } \\
\text { ich }\end{array}$ & $\begin{array}{l}\text { nicht } \\
\text { wie } \\
\text { ich }\end{array}$ & $\begin{array}{l}\text { über- } \\
\text { haupt } \\
\text { nicht } \\
\text { wie } \\
\text { ich }\end{array}$ \\
\hline $\begin{array}{l}\text { 9. Sie/Er denkt, dass es wichtig ist } \\
\text { nicht mehr zu verlangen als man hat. } \\
\text { Sie/Er meint, dass Menschen mit dem } \\
\text { zufrieden sein sollen, was sie haben. }\end{array}$ & $\square$ & $\square$ & $\square$ & $\square$ & $\square$ & $\square$ \\
\hline $\begin{array}{l}\text { 10. SielEr sucht nach jeder } \\
\text { Möglichkeit, Spaß zu haben. Es ist } \\
\text { wichtig für sielihn, Dinge zu tun, die } \\
\text { ihrlihm Vergnügen bereiten. }\end{array}$ & $\square$ & $\square$ & $\square$ & $\square$ & $\square$ & $\square$ \\
\hline $\begin{array}{l}\text { 11. Es ist wichtig für sielihn, selbst zu } \\
\text { entscheiden was sieler tut. SielEr } \\
\text { möchte ihrelseine eigenen Aktivitäten } \\
\text { frei planen und auswählen können. }\end{array}$ & $\square$ & $\square$ & $\square$ & $\square$ & $\square$ & $\square$ \\
\hline $\begin{array}{l}\text { 12. Es ist sehr wichtig für sielihn, den } \\
\text { Menschen um ihrlihn herum zu helfen. } \\
\text { SielEr möchte sich um ihr Wohlergehen } \\
\text { kümmern. }\end{array}$ & $\square$ & $\square$ & $\square$ & $\square$ & $\square$ & $\square$ \\
\hline $\begin{array}{l}\text { 13. Sehr erfolgreich zu sein ist für } \\
\text { sielihn wichtig. SielEr möchte andere } \\
\text { Menschen beeindrucken. }\end{array}$ & $\square$ & $\square$ & $\square$ & $\square$ & $\square$ & $\square$ \\
\hline $\begin{array}{l}\text { 14. Es ist sehr wichtig für sielihn, dass } \\
\text { ihr/sein Land sicher ist. SielEr denkt, } \\
\text { dass der Staat gegenüber äußeren und } \\
\text { inneren Bedrohungen wachsam sein } \\
\text { muss. }\end{array}$ & $\square$ & $\square$ & $\square$ & $\square$ & $\square$ & $\square$ \\
\hline $\begin{array}{l}\text { 15. SielEr geht gerne Risiken ein. } \\
\text { SielEr ist immer auf der Suche nach } \\
\text { Abenteuern. }\end{array}$ & $\square$ & $\square$ & $\square$ & $\square$ & $\square$ & $\square$ \\
\hline $\begin{array}{l}\text { 16. Es ist für sielihn wichtig, sich immer } \\
\text { angemessen zu verhalten. SielEr } \\
\text { möchte vermeiden, irgend etwas zu tun, } \\
\text { von dem die Leute sagen könnten, dass } \\
\text { es falsch sei. }\end{array}$ & $\square$ & $\square$ & $\square$ & $\square$ & $\square$ & $\square$ \\
\hline $\begin{array}{l}\text { 17. Es ist wichtig für sie/ihn, die } \\
\text { Leitung zu haben und anderen zu sagen } \\
\text { was sie zu tun haben. SielEr möchte, } \\
\text { dass Menschen das tun, was sieler sagt. }\end{array}$ & $\square$ & $\square$ & $\square$ & $\square$ & $\square$ & $\square$ \\
\hline $\begin{array}{l}\text { 18. Für sielihn ist es wichtig, ih- } \\
\text { ren/seinen Freunden treu zu sein. } \\
\text { Sie/Er möchte sich den Menschen wid- } \\
\text { men, die ihr/ihm nahe stehen. }\end{array}$ & $\square$ & $\square$ & $\square$ & $\square$ & $\square$ & $\square$ \\
\hline $\begin{array}{l}\text { 19. Sie/Er ist sehr davon überzeugt, } \\
\text { dass Menschen die Natur schützen sol- } \\
\text { len. Die Umwelt zu schützen ist sehr } \\
\text { wichtig für sielihn. }\end{array}$ & $\square$ & $\square$ & $\square$ & $\square$ & $\square$ & $\square$ \\
\hline
\end{tabular}




\begin{tabular}{|c|c|c|c|c|c|c|}
\hline & $\begin{array}{l}\text { sehr } \\
\text { wie } \\
\text { ich }\end{array}$ & $\begin{array}{l}\text { wie } \\
\text { ich }\end{array}$ & $\begin{array}{l}\text { etwa } \\
\text { wie } \\
\text { ich }\end{array}$ & $\begin{array}{l}\text { ein } \\
\text { wenig } \\
\text { wie } \\
\text { ich }\end{array}$ & $\begin{array}{l}\text { nicht } \\
\text { wie } \\
\text { ich }\end{array}$ & $\begin{array}{l}\text { über- } \\
\text { haupt } \\
\text { nicht } \\
\text { wie } \\
\text { ich }\end{array}$ \\
\hline $\begin{array}{l}\text { 20. Religiös zu sein ist wichtig für } \\
\text { sie/ihn. SielEr bemüht sich sehr, nach } \\
\text { ihren/seinen religiösen Überzeugungen } \\
\text { zu leben. }\end{array}$ & $\square$ & $\square$ & $\square$ & $\square$ & $\square$ & $\square$ \\
\hline $\begin{array}{l}\text { 21. Es ist für sielihn wichtig, dass Dinge } \\
\text { in Ordnung und sauber sind. SielEr mag } \\
\text { es wirklich überhaupt nicht, wenn Dinge } \\
\text { in Unordnung sind. }\end{array}$ & $\square$ & $\square$ & $\square$ & $\square$ & $\square$ & $\square$ \\
\hline $\begin{array}{l}\text { 22. Sie/Er denkt, dass es wichtig ist, } \\
\text { sich für viele Sachen zu interessieren. } \\
\text { SielEr ist gerne neugierig und versucht, } \\
\text { alle möglichen Dinge zu verstehen. }\end{array}$ & $\square$ & $\square$ & $\square$ & $\square$ & $\square$ & $\square$ \\
\hline $\begin{array}{l}\text { 23. SielEr glaubt, dass alle Menschen } \\
\text { auf der Welt in Harmonie miteinander } \\
\text { leben sollten. Zwischen allen Menschen- } \\
\text { gruppen auf der Welt Frieden herzu- } \\
\text { stellen ist für sielihn sehr wichtig. }\end{array}$ & $\square$ & $\square$ & $\square$ & $\square$ & $\square$ & $\square$ \\
\hline $\begin{array}{l}\text { 24. SielEr denkt, dass es wichtig ist, } \\
\text { ehrgeizig zu sein. SielEr möchte zeigen, } \\
\text { wie fähig sieler ist. }\end{array}$ & $\square$ & $\square$ & $\square$ & $\square$ & $\square$ & $\square$ \\
\hline $\begin{array}{l}\text { 25. Sie/Er denkt, dass es das Beste ist, } \\
\text { Dinge auf die traditionelle Art und Wei- } \\
\text { se zu tun. Für sielihn ist es wichtig, die } \\
\text { Bräuche zu erhalten, die sieler gelernt } \\
\text { hat. }\end{array}$ & $\square$ & $\square$ & $\square$ & $\square$ & $\square$ & $\square$ \\
\hline $\begin{array}{l}\text { 26. Für sielihn ist es wichtig, die } \\
\text { Freuden des Lebens zu genießen. Sie/Er } \\
\text { 'verwöhnt' sich gerne selbst. }\end{array}$ & $\square$ & $\square$ & $\square$ & $\square$ & $\square$ & $\square$ \\
\hline $\begin{array}{l}\text { 27. Es ist wichtig für sielihn, auf die } \\
\text { Bedürnisse anderer zu achten. SielEr } \\
\text { versucht Menschen, die sieler kennt, zu } \\
\text { unterstützen. }\end{array}$ & $\square$ & $\square$ & $\square$ & $\square$ & $\square$ & $\square$ \\
\hline $\begin{array}{l}\text { 28. SielEr ist davon überzeugt, dass } \\
\text { sieler ihrelseine Eltern und ältere } \\
\text { Menschen immer mit Respekt behandeln } \\
\text { soll. Es ist für sielihn wichtig, gehorsam } \\
\text { zu sein. }\end{array}$ & $\square$ & $\square$ & $\square$ & $\square$ & $\square$ & $\square$ \\
\hline $\begin{array}{l}\text { 29. Sie/Er möchte, dass jede/r gerecht } \\
\text { behandelt wird, auch Menschen, die } \\
\text { sieler nicht kennt. Für sielihn ist es } \\
\text { wichtig, die Schwachen in der Gesell- } \\
\text { schaft zu beschützen. }\end{array}$ & $\square$ & $\square$ & $\square$ & $\square$ & $\square$ & $\square$ \\
\hline
\end{tabular}




\begin{tabular}{|c|c|c|c|c|c|c|}
\hline & $\begin{array}{l}\text { sehr } \\
\text { wie } \\
\text { ich }\end{array}$ & $\begin{array}{l}\text { wie } \\
\text { ich }\end{array}$ & $\begin{array}{l}\text { etwa } \\
\text { wie } \\
\text { ich }\end{array}$ & $\begin{array}{l}\text { ein } \\
\text { wenig } \\
\text { wie } \\
\text { ich }\end{array}$ & $\begin{array}{l}\text { nicht } \\
\text { wie } \\
\text { ich }\end{array}$ & $\begin{array}{l}\text { über- } \\
\text { haupt } \\
\text { nicht } \\
\text { wie } \\
\text { ich }\end{array}$ \\
\hline $\begin{array}{l}\text { 30. SielEr mag Überraschungen. Es ist } \\
\text { wichtig für sielihn, ein aufregendes } \\
\text { Leben zu haben. }\end{array}$ & $\square$ & $\square$ & $\square$ & $\square$ & $\square$ & $\square$ \\
\hline $\begin{array}{l}\text { 31. Sie/Er bemüht sich sehr, nicht } \\
\text { krank zu werden. Gesund zu bleiben ist } \\
\text { für sie/ihn sehr wichtig. }\end{array}$ & $\square$ & $\square$ & $\square$ & $\square$ & $\square$ & $\square$ \\
\hline $\begin{array}{l}\text { 32. Im Leben vorwärts zu kommen ist } \\
\text { für sielihn wichtig. SielEr strebt } \\
\text { danach, besser als andere zu sein. }\end{array}$ & $\square$ & $\square$ & $\square$ & $\square$ & $\square$ & $\square$ \\
\hline $\begin{array}{l}\text { 33. Menschen zu vergeben, die sielihn } \\
\text { verletzt haben, ist wichtig für sielihn. } \\
\text { SielEr bemüht sich, das Gute an ihnen } \\
\text { zu sehen und keinen Groll gegen sie zu } \\
\text { hegen. }\end{array}$ & $\square$ & $\square$ & $\square$ & $\square$ & $\square$ & $\square$ \\
\hline $\begin{array}{l}\text { 34. Es ist wichtig für sielihn, } \\
\text { unabhängig zu sein. SielEr verlässt sich } \\
\text { gerne auf sich selbst. }\end{array}$ & $\square$ & $\square$ & $\square$ & $\square$ & $\square$ & $\square$ \\
\hline $\begin{array}{l}\text { 35. Für sielihn ist es wichtig, dass es } \\
\text { eine stabile Regierung gibt. SielEr ist } \\
\text { daran interessiert, dass die soziale } \\
\text { Ordnung geschützt wird. }\end{array}$ & $\square$ & $\square$ & $\square$ & $\square$ & $\square$ & $\square$ \\
\hline $\begin{array}{l}\text { 36. Es ist wichtig für sielihn, anderen } \\
\text { Menschen gegenüber immer höflich zu } \\
\text { sein. SielEr bemüht sich, andere nie zu } \\
\text { stören oder zu verärgern. }\end{array}$ & $\square$ & $\square$ & $\square$ & $\square$ & $\square$ & $\square$ \\
\hline $\begin{array}{l}\text { 37. Sie/Er möchte das Leben wirklich } \\
\text { genießen. Eine gute Zeit zu haben ist } \\
\text { für sielihn sehr wichtig. }\end{array}$ & $\square$ & $\square$ & $\square$ & $\square$ & $\square$ & $\square$ \\
\hline $\begin{array}{l}\text { 38. Es ist wichtig für sielihn, an- } \\
\text { spruchslos und bescheiden zu sein. } \\
\text { SielEr versucht nicht die Aufmerksam- } \\
\text { keit anderer zu erregen. }\end{array}$ & $\square$ & $\square$ & $\square$ & $\square$ & $\square$ & $\square$ \\
\hline $\begin{array}{l}\text { 39. SielEr möchte immer dielder sein, } \\
\text { dielder die Entscheidungen trifft. } \\
\text { SielEr mag es, Leute zu führen. }\end{array}$ & $\square$ & $\square$ & $\square$ & $\square$ & $\square$ & $\square$ \\
\hline $\begin{array}{l}\text { 40. Es ist wichtig für sie/ihn, sich nach } \\
\text { der Natur zu richten und sich an sie } \\
\text { anzupassen. SielEr ist davon überzeugt, } \\
\text { dass Menschen nicht die Natur verän- } \\
\text { dern sollen. }\end{array}$ & $\square$ & $\square$ & $\square$ & $\square$ & $\square$ & $\square$ \\
\hline
\end{tabular}

Vielen Dank für Ihre Mitarbeit!

Das Fragebogen Team 



\section{Abstract}

Most international legal frameworks that aim at protecting biodiversity place great importance on educational measures (e.g. The Convention on Biological Diversity (CBD), Art. 13a). However, some of the world's regions are more affected by biodiversity loss than others (which are so-called biodiversity hotspots). Resource dilemmas are serious threats to biodiversity, especially in such regions. In resource dilemmas, natural resources are depleted for economic interest, which is, in many cases, required income. Therefore, economic and social aspects must be considered should biodiversity loss be completely understood. However, little is known thus far about learning prerequisites of pupils living in an industrial country, such as Germany, and in a biodiversity hotspot, such as Chile. This dissertation focuses on two important learning prerequisites being: i) pupils’ perceptions of biodiversity, biodiversity loss and resource dilemmas, and ii) pupils' commitment to protect biodiversity. Firstly, a qualitative, in-depth interview study was conducted that investigated sixteen to eighteen-year-old Chilean and German learners' perception of biodiversity and its loss $(n=24)$. As a central result, an "ecological type”, an "economic type” and a "balanced type” emerged from the data, showing different perspectives of biodiversity and its loss. Overall, Chilean pupils seemed to have greater difficulties in recognising social aspects of biodiversity loss. Surprisingly, pupils who concentrated on ecological aspects, applied weak ecological knowledge more often. However, some pupils did succeed in integrating ecological, economic, and social aspects. We suggest that interdisciplinary treatment of the subject matter "biodiversity" is indispensable if learning about sustainable development and ecological learning is to be effective.

Secondly, Chilean and German pupils’ commitments to protect biodiversity were explored. Based on the Value-Belief-Norm theory, a questionnaire study was conducted with Chilean $(\mathrm{n}=216)$ and German $(\mathrm{n}=217) 15$ to 19-year-old pupils. Comparisons revealed that Chilean adolescents showed overall higher personal norms and commitments to protect biodiversity. Furthermore, regression analyses showed that within the German sample, the human value of universalism was an important predictor for behavioural commitment. In addition, in both samples, ascription of responsibility, perceived ability to reduce threats and, above all, personal norms were positive predictors. As one consequence of biodiversity education, pupils should be provided with convincing behavioural options that also withstand critical questions with respect to their effectivity. A valuable starting point would be environmental performance evaluations of everyday-life products and a consistent reflection of consumer's own behaviour. 


\section{Zusammenfassung}

Zahlreiche internationale rechtliche Bestimmungen zum Schutz der Biodiversität betonen den zentralen Stellenwert von Bildung (z.B. Konvention über die biologische Vielfalt (CBD), Art. 13a). An sogenannten Biodiversitäts-Hotspots, an denen die Biodiversität besonders gefährdet ist, stellen ökologisch-soziale Dilemmata eine Bedrohung für die Biodiversität dar. Dabei werden natürliche Ressourcen aus ökonomischem Interesse, das häufig in der Befriedigung von Grundbedürfnissen besteht, übernutzt. Ökonomische und soziale Aspekte müssen daher in eine Bildung zur Biodiversität einbezogen werden, wenn das Problem des Biodiversitätsverlusts adäquat rekonstruiert werden soll. Über Lernvoraussetzungen zur Biodiversität von Schüler(inne)n, die in einem Industrieland wie Deutschland und an einem Biodiversitäts-Hotspot wie Chile leben, ist wenig bekannt. Die vorliegende Dissertation nimmt zwei Lernvoraussetzungen zur Biodiversität in den Blick: 1. Die Wahrnehmung von Biodiversität, Biodiversitätsverlust und ökologisch-sozialen Dilemmata und 2. die Bereitschaft von Schüler(inne)n, die Biodiversität zu schützen.

Zunächst wurde eine qualitative Interviewstudie mit $(n=24)$ 16-17jährigen chilenischen und deutschen Schüler(inne)n durchgeführt, um deren Wahrnehmung von Biodiversität und Biodiversitätsverlust zu untersuchen. Als zentrales Ergebnis konnten ein „ökologischer Typ“, ein „ökonomischer Typ“ und ein „ausgeglichener Typ“ identifiziert werden, die sich in ihren Argumentationen unterschieden. Insgesamt bezogen chilenische Schüler(innen) soziale Aspekte des Biodiversitätsverlusts weniger in ihre Überlegungen ein. Zudem zeigten die Schüler(innen), die vornehmlich auf ökologische Aspekte fokussierten, eine Tendenz zur Anwendung negativen Wissens. Die Ergebnisse zeigen, dass eine interdisziplinäre Betrachtung des Themas Biodiversität wichtig ist für ein Lernen im Sinne Nachhaltiger Entwicklung und für ein erfolgreiches Verstehen von ökologischen Aspekten.

Als ein zweiter Fokus wurden Bereitschaften untersucht, die Biodiversität zu schützen. Basierend auf der Value-Belief-Norm Theorie wurde eine Fragebogenstudie mit 216 chilenischen und 217 deutschen 15-19jährigen Schüler(inne)n durchgeführt. Chilenische Schüler(innen) zeigten insgesamt höhere persönliche Normen und Bereitschaften, die Biodiversität zu schützen. In beiden Stichproben waren die wahrgenommene Verantwortung, die wahrgenommene Möglichkeit, Bedrohungen für die Biodiversität einzudämmen und, vor allem, persönliche Normen wichtige positive Prädiktoren für Schutzbereitschaften. Biodiversitätsbildung sollte Schüler(inne)n daher realistische Handlungsoptionen aufzeigen. Ansatzpunkte sind sogenannte „Ökobilanzen“, durch die Produkte des täglichen Leben und damit das eigene Konsumverhalten kritisch reflektiert werden können. 


\section{Lebenslauf}

Susanne Menzel, M.A.

Emilienstr. 13

37075 Göttingen

susanne.menzel@gwdg.de

0174-7472854

Name

Susanne Menzel

Geburtsdatum und -ort

8. Juni 1976 in Siegen

Staatsangehörigkeit deutsch

1995

1996-2002

1999-2000

2000-2001

2002

2003

2003

Seit 2004
Abitur

Studium der Fächer Biologie, Pädagogik und Englisch, Westfälische-Wilhelms-Universität Münster

Auslandsstudium, Université Cheikh Anta Diop, Dakar, Senegal

Auslandsstudium, College of William and Mary, Virginia, U.S.A.

- Erstes Staatsexamen für Lehrämter der Sekundarstufen I und II; Unterrichtsfächer: Biologie und Pädagogik

- Magistra Artium (M.A.); Hauptfach: Pädagogik, Nebenfächer: Biologie und Englisch

Wissenschaftliche Hilfskraft, Westfälische-Wilhelms-Universität Münster, Fachbereich Erziehungswissenschaften

Wissenschaftliche Mitarbeiterin, Zentrum für Umweltforschung (ZUFO), Westfälische-Wilhelms-Universität Münster

Wissenschaftliche Mitarbeiterin, Didaktik der Biologie, GeorgAugust-Universität Göttingen

Fremdsprachen

Englisch, Französisch, Spanisch

Wissenschaftliche

Tätigkeiten im Ausland (Auswahl)
- 2004, 2005, 2006 einmonatige Forschungsaufenthalte, qualitative und quantitative Datenerhebung, Talca (Chile)

- 2006 Gastvorträge Universidad del Azuay, Cuenca (Ecuador)

- 2006 Großpraktikum, Iquitos (Peru) in Zusammenarbeit mit dem Deutschen Primatenzentrum und der Universidad de la Amazonía

- 2002 sechswöchige Recherchen (Magisterarbeit), Südafrika und Namibia 